\title{
LINKED TREE-DECOMPOSITIONS OF INFINITE REPRESENTED MATROIDS
}

\author{
By \\ Jeffrey Donald Azzato
}

A thesis

submitted to the Victoria University of Wellington in fulfilment of the requirements for the degree of Master of Science in Mathematics

Victoria University of Wellington 2008 



\begin{abstract}
It is natural to try to extend the results of Robertson and Seymour's Graph Minors Project to other objects. As linked tree-decompositions (LTDS) of graphs played a key role in the Graph Minors Project, establishing the existence of LTDS of other objects is a useful step towards such extensions. There has been progress in this direction for both infinite graphs and matroids. Kř́ž and Thomas proved that infinite graphs of finite tree-width have LTDs. More recently, Geelen, Gerards and Whittle proved that matroids have linked branch-decompositions, which are similar to LTDS. These results suggest that infinite matroids of finite treewidth should have LTDS.

We answer this conjecture affirmatively for the representable case. Specifically, an independence space is an infinite matroid, and a point configuration (hereafter configuration) is a represented independence space. It is shown that every configuration having tree-width $k \in \omega$ has an LTD of width at most $2 k$.

Configuration analogues for bridges of $X$ (also called connected components modulo $\mathrm{X}$ ) and chordality in graphs are introduced to prove this result. A correspondence is established between chordal configurations only containing subspaces of dimension at most $k \in \omega$ and configuration tree-decompositions having width at most $k$. This correspondence is used to characterise finite-width LTDS of configurations by their local structure, enabling the proof of the existence result. The theory developed is also used to show compactness of configuration tree-width: a configuration has tree-width at most $k \in \omega$ if and only if each of its finite subconfigurations has tree-width at most $k \in \omega$.

The existence of LTDs for configurations having finite tree-width opens the possibility of well-quasi-ordering (or even better-quasi-ordering) by minors those independence spaces representable over a fixed finite field and having bounded tree-width.
\end{abstract}




\section{Acknowledgments}

I wish to extend my thanks to all those who have helped and supported me during my studies. First of all, I am very grateful to my supervisor Prof. Geoff Whittle, both for first introducing me to matroids and for offering lots of encouragement, sound suggestions, and insights into the world of research during my work on this thesis. I also wish to thank Prof. Rod Downey for suggesting the better-quasi-ordering problem that motivates much of this thesis, and the VUW Research Fund for its generous support.

During the last year I have had the pleasure of many mathematical discussions with both other members of our department and visitors. I hope that they found these as interesting as I did. Also, late last year I was able to attend the 32nd Australasian Conference on Combinatorial Mathematics and Combinatorial Computing through a VUW Science Faculty Small Research Grant, for which I am grateful.

Finally, many heartfelt thanks are due my family for not one, but many years of unflagging support in all my studies, and their help in proofing drafts of this manuscript. 


\section{Contents}

1 Introduction $\quad 1$

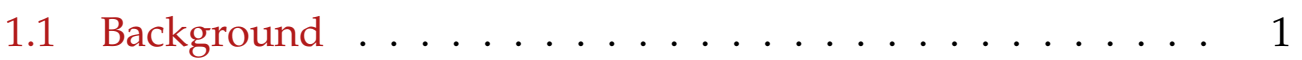

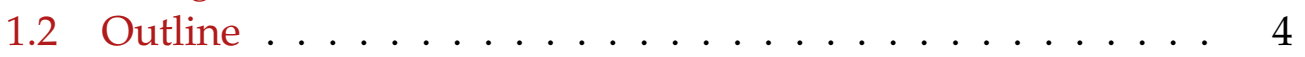

1.3 Pre-Existing and New Material ........... 5

2 Preliminaries $\quad 7$

2.1 Graph Theory . . . . . . . . . . . . . . . . 7

2.2 Linear Algebra . . . . . . . . . . . . . . . . . . . . . . . . 11

2.3 Rado's Selection Lemma . . . . . . . . . . . . . . . . . 14

3 Independence Spaces $\quad 17$

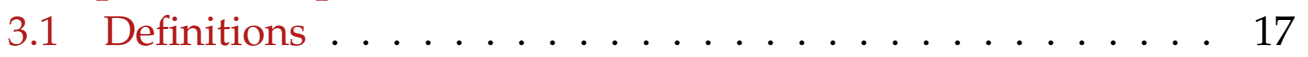

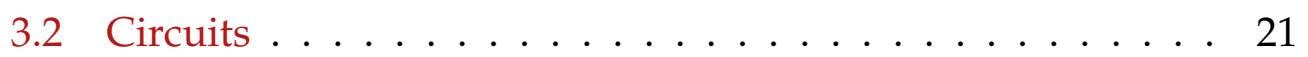

3.3 Bases and Restriction . . . . . . . . . . . . . 23

3.4 Finitary Rank . . . . . . . . . . . . . . . . . . . 26

3.5 Closure and Flats . . . . . . . . . . . . . . 33

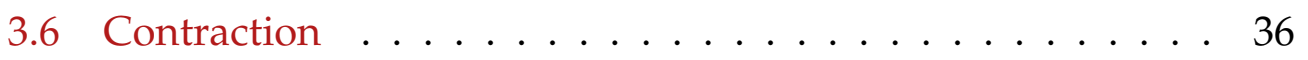

3.7 Simplification . . . . . . . . . . . . . . . . . . 41

3.8 Geometric Representations . . . . . . . . . . . . . . . . . . 42

3.9 Connectivity . . . . . . . . . . . . . . . 44

4 Configurations $\quad 49$

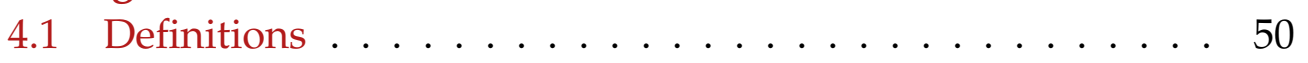

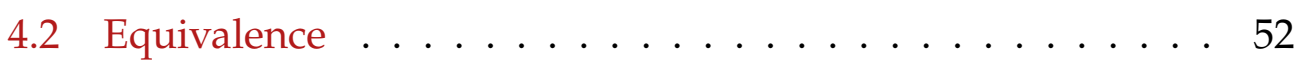

4.3 Restriction . . . . . . . . . . . . . . 57

4.4 Contraction ....................... 59

4.5 Connectivity ...................... 65 
5 Further Notions $\quad 69$

5.1 Bridges . . . . . . . . . . . . . . . . 69

5.2 Roundness . . . . . . . . . . . . . . . . 80

5.3 Chordal Saturation . . . . . . . . . . . . . 88

6 Tree-Decompositions of Configurations $\quad 97$

6.1 Definitions and Elementary Results . . . . . . . . . . . 97

6.2 Linked Decompositions: The Finite Case . . . . . . . . . . . 113

6.3 Roundness and Tree-Decompositions . . . . . . . . . . . . 121

6.4 Chordal Saturation and Tree-Decompositions . . . . . . . . 125

7 Linked Decompositions: The Infinite Case 139

8 Discussion $\quad 145$

$\begin{array}{ll}\text { Bibliography } & 149\end{array}$

$\begin{array}{ll}\text { Notation } & 154\end{array}$

$\begin{array}{ll}\text { Index } & 156\end{array}$ 


\section{Chapter 1}

\section{Introduction}

\subsection{Background}

Trees are among the simplest graphs. Many results that are difficult in general are easily established for trees-a property that makes the study of tree-like objects very attractive. A tree-decomposition of an object is a decomposition of that object into parts of bounded size such that the parts fit together in a tree-like way. This thesis is primarily devoted to establishing the existence of linked tree-decompositions of "infinite represented matroids" having finite tree-width.

Tree-decompositions and tree-width of graphs were first introduced (under different names) by Halin in [Hal76], ${ }^{1}$ although they did not gain widespread currency until Robertson and Seymour gave their definition in [RS90]. Graph tree-width is an invariant that measures how tree-like a graph is: the more tree-like the graph, the smaller its tree-width. For example, the simple graphs having tree-width at most 1 are precisely the forests.

Tree-decompositions of graphs play an important role in Robertson and Seymour's proof in [RS04] of the following deep theorem, a survey

\footnotetext{
${ }^{1}$ See p. 354 of [Die06].
} 
of which may be found in [Lov05].

Graph Minor Theorem (Robertson and Seymour). Finite graphs are wellquasi-ordered under the minor relation.

Robertson and Seymour also established the existence of a cubic-time algorithm for testing whether a finite graph $\mathrm{G}$ has an H-minor (see p. 66 of [RS95]). Combining this result with the Graph Minor Theorem gives the next theorem.

Theorem 1.1.1 (Robertson and Seymour). Every minor-closed property of finite graphs can be tested in cubic time.

Unusually, this theorem is purely existential: it does not explicitly provide algorithms for testing minor-closed properties of graphs. However, the assurance that polynomial-time algorithms exist for testing such properties has generated widespread interest. Tree-width has proven useful in developing good algorithms-indeed, many NP-hard graph parameters can be computed in polynomial time for graphs having bounded tree-width. This has spurred investigation of related invariants, such as branch-width, clique-width and hypertree-width. These "width parameters" and their algorithmic applications are surveyed in [HOSG07].

Extensions of parts of the Graph Minors Project to other objects have also been pursued. Thomas proved the following theorem in [Tho89a].

Theorem 1.1.2 (Thomas). Every class of graphs having bounded tree-width is well-behaved under the minor relation.

"Well-behaved" is a technical strengthening of Nash-Williams' concept of "better-quasi-ordered" (introduced in [NW68]), which is in turn considerably stronger than "well-quasi-ordered." Bounded tree-width is necessary here: Thomas also gave a counterexample in [Tho88] showing that graphs in general are not well-quasi-ordered under the minor relation. $^{2}$

\footnotetext{
${ }^{2}$ The counterexample uses uncountable graphs, and it remains open whether countable graphs are well-quasi-ordered or not.
} 
More recently, Geelen, Gerards and Whittle established the following theorem in [GGW02].

Theorem 1.1.3 (Geelen, Gerards and Whittle). Every class of matroids representable over a fixed finite field and having bounded branch-width is well-quasiordered under the minor relation.

Branch-width is an invariant closely related to tree-width. In particular, the branch-width of a matroid is bounded if and only if its treewidth is bounded (see pp. 1122-1123 of [HW06]), so Theorem 1.1.3 still holds when "branch-width" is replaced by "tree-width." Geelen, Gerards and Whittle also demonstrated in [GGW02] that finiteness of the field is necessary - a reflection of the greater generality of the matroidal setting.

Taken together, Theorems 1.1.2 and 1.1.3 suggest that the following conjecture is likely to hold.

Conjecture 1.1.4. Every class of independence spaces representable over a fixed finite field and having bounded tree-width is well-quasi-ordered under the minor relation.

Independence spaces are one of several generalisations of matroids to infinite sets. Unlike other generalisations, they

- generalise graphs in the same way that matroids generalise finite graphs, and

- only have finite circuits in the same way that graphs only have finite cycles.

These properties make independence spaces the appropriate choice of "infinite matroid" for Conjecture 1.1.4.

The proofs of Theorems 1.1.2 and 1.1.3 each rely on the existence of linked decompositions. A (tree- or branch-) decomposition of an object is linked if the connectivity between any pair of the object's parts displayed by the decomposition is precisely the least width appearing between them 
in the decomposition. Linked decompositions certify that certain minors exist, while bounded (tree- or branch-) width limits their number. Together these conditions prevent the existence of a minor-minimal bad sequence, thus demonstrating that well-quasi-ordering holds.

The existence of linked tree-decompositions of graphs having finite tree-width was established by Thomas and Kř́ž. Thomas closed the finite case in [Tho90].

Theorem 1.1.5 (Thomas). Every finite graph having tree-width $w$ has a linked tree-decomposition of width $w$.

Using the Axiom of Choice and a compactness argument, Kříž and Thomas were subsequently able to lift this result to infinite graphs in [KT91].

Theorem 1.1.6 (Křy̌ž and Thomas). Every graph having tree-width $w \in \omega$ has a linked tree-decomposition of width $w$.

This thesis is primarily devoted to establishing an analogue of Theorem 1.1.6 for point configurations, which are represented independence spaces. The structure of the argument given mirrors that of Kř́žz and Thomas.

\subsection{Outline}

This thesis is arranged as follows. Chapter 2 introduces some background graph theory and linear algebra, as well as a combinatorially-useful form of the Axiom of Choice.

As many of the results needed for point configurations hold for independence spaces in general, it is sensible to establish them for independence spaces. Chapter 3 is devoted to this.

Chapter 4 formally introduces point configurations and develops the relationship between these and general independence spaces. This allows 
the results of Chapter 3 to be applied in the context of point configurations.

Chapter 5 explores the more exotic concepts of bridges, roundness and chordal saturation. Each motivated by an analogue in graph theory, these concepts play a vital role in the generalisation of Theorem 1.1.6.

Tree-decompositions of point configurations are presented in Chapter 6. Section 6.1 establishes some elementary properties of tree-decompositions. An analogue of Theorem 1.1.5 for point configurations is proved in Section 6.2. Sections 6.3 and 6.4 develop the relationships between roundness, chordal saturation and tree-decompositions, providing lemmas later crucial to the proof of the main theorem. Compactness of treewidth for point configurations is established at the end of Section 6.4.

Chapter 7 is devoted to the proof of the main theorem. Finally, Chapter 8 suggests possible future avenues of research.

\subsection{Pre-Existing and New Material}

Here we note which results are pre-existing, and which are new.

With the exceptions of Lemmas 2.2.1 and 2.2.7 (the first of which is obvious), all results in Chapter 2 are pre-existing. While Proposition 2.1.3 was already known, we are unaware of any proof in the literature.

The results in Chapter 3 are either pre-existing or straightforward given pre-existing results.

As Chapter 4 is devoted to configuration-specific formulations of matroidal concepts, many of its results are unsurprising. The material in Section 4.1 is all pre-existing. Most material in Sections 4.2-4.5 is wellknown in the context of matroids. However, Definition 4.2.1, Propositions 4.2.2 and 4.2.3, Definition 4.4.1 and Proposition 4.4.2, while similar to some pre-existing work, appear to be new. Proposition 4.4.7 is new.

Chapter 5 introduces some new concepts, and thus contains new results. The "bridges" of Definition 5.1.3 are new. Consequently most of 
the results of Section 5.1, while straightforward, are new. Definition 5.1.1 and Proposition 5.1.2 are as for matroids, and several other results in this section are natural generalisations of matroid results. In Section 5.2, both Proposition 5.2.7 and Corollary 5.2.8 are new. All other material in this section is pre-existing for matroids, although here it is generalised to independence spaces. Section 5.3 is entirely new.

With the exception of Definition 6.1.2, all of the material in Chapters 6 and 7 is new. 


\section{Chapter 2}

\section{Preliminaries}

We shall assume a number of elementary results, but review the most relevant material in this chapter.

\subsection{Graph Theory}

We first review some standard definitions and notations for graphs and trees.

A graph $G$ is a triple $(V, E, l)$ consisting of a set $V$, a set $E$ disjoint from $V$ and a function $\mathrm{l}: \mathrm{E} \rightarrow\left\{\mathrm{V}^{\prime} \subseteq \mathrm{V}|1 \leqslant| \mathrm{V}^{\prime} \mid \leqslant 2\right\}$. The vertex set of $\mathrm{G}$, denoted $V(G)$, is $V$, and the vertices of $G$ are the elements of $V$. Similarly, the edge set of $G$, denoted $E(G)$, is $E$, and the edges of $G$ are the elements of $E$. The order of $G$, denoted $|G|$, is $|V|$. We denote $|E(G)|$ by e $(G)$. A graph is finite if both its vertex and edge sets are finite, and empty if its vertex set is empty.

We shall usually only refer to the incidence relation $\iota$ implicitly, writing "the graph $(\mathrm{V}, \mathrm{E})$," etc. Moreover, we shall identify each edge $e \in E$ with $\iota(e)$, writing $v v^{\prime}$ to mean $e$ whenever $\iota(e)=\left\{v, v^{\prime}\right\}$ and $v v$ to mean $e$ whenever $\iota(e)=\{v\}$.

A vertex $v \in \mathrm{V}$ is incident on an edge $e \in E$ (and vice versa) if $v \in \mathfrak{l}(e)$. Moreover, $v$ is incident on $E^{\prime} \subseteq E$ if $v$ is incident on some $e^{\prime} \in E^{\prime}$, and 
$e$ is incident on $V^{\prime} \subseteq V$ if $e$ is incident on some $v^{\prime} \in V^{\prime}$. The set of vertices $U_{l}\left[E^{\prime}\right]$ of $G$ incident on $E^{\prime}$ is denoted $V_{G}\left(E^{\prime}\right)$, while the set of edges $\left\{e \in E \mid V^{\prime} \cap \mathfrak{\imath}(e) \neq \varnothing\right\}$ of $G$ incident on $V^{\prime}$ is denoted $E_{G}\left(V^{\prime}\right)$.

A loop is an edge incident on only one vertex. A pair of distinct nonloop edges are parallel if they are incident on the same pair of vertices. A vertex $v \in \mathrm{V}$ is a neighbour of a vertex $v^{\prime} \in \mathrm{V}$ in $\mathrm{G}$ if $v \neq v^{\prime}$ and there exists an edge of $\mathrm{G}$ incident on both. The set of neighbours of $v$ in $\mathrm{G}$ is denoted $\mathrm{N}_{\mathrm{G}}(v)$.

The degree of a vertex $v$ of $G$, denoted $d_{G}(v)$, is the number of edges of $\mathrm{G}$ incident on $v$, with loops counting twice. This is taken to be (symbolically) $\infty$ if it is not finite. The maximum degree of $\mathrm{G}$ is

$$
\Delta(\mathrm{G}):=\sup \left\{\mathrm{d}_{\mathrm{G}}(v) \mid v \in \mathrm{V}\right\}
$$

A graph $G^{\prime}=\left(V^{\prime}, E^{\prime}, \iota^{\prime}\right)$ is a subgraph of a graph $G=(V, E, l)$, denoted $\mathrm{G}^{\prime} \leqslant \mathrm{G}$, if $\mathrm{V}^{\prime} \subseteq \mathrm{V}$ and $\mathrm{E}^{\prime} \subseteq \mathrm{E}$ and $\mathrm{l}_{\mathrm{E}^{\prime}}=\mathrm{l}^{\prime}$. A path $\mathrm{P}$ in a graph $\mathrm{G}$ is a subgraph $\left(\left\{v_{1}, \ldots, v_{n}\right\},\left\{e_{1}, \ldots, e_{n-1}\right\}, \iota^{\prime}\right)$ of $G$ having $\iota^{\prime}\left(e_{i}\right)=\left\{v_{i}, v_{i+1}\right\}$ for each $i=1, \ldots, n-1$. Sometimes we shall identify $P$ with the sequence

$$
v_{1}, e_{1}, v_{2}, e_{2}, \ldots, e_{n-1}, v_{n}
$$

of its vertices and edges. We say that $\mathrm{P}$ links $v_{1}$ and $v_{n}$, which are its endvertices (note that some authors call an endvertex an "end"). A graph is connected if every pair of its vertices are linked by a path.

A cycle of a graph $\mathrm{G}$ is a finite non-empty connected subgraph of $\mathrm{G}$ for which every vertex has degree 2 . A forest is an acyclic graph, and a tree is a connected forest. If $T$ is a tree and $t, t^{\prime} \in V(T)$, there exists a unique path in $T$ linking $t$ and $t^{\prime}$, denoted $t T t^{\prime}$. A leaf is a vertex of a tree having degree 1. The set of leaves of a tree $T$ is denoted $L(T)$. A tree is cubic if each of its non-leaf vertices has degree 3.

The union of graphs $G=(V, E, l)$ and $G^{\prime}=\left(V^{\prime}, E^{\prime}, \iota^{\prime}\right)$ having $V \cap E^{\prime}=$ 
$V^{\prime} \cap E=\varnothing$ and $\iota_{E \cap E^{\prime}}=\left.\iota^{\prime}\right|_{E \cap E^{\prime}}$, denoted $G \cup G^{\prime}$, is the graph $\left(V \cup V^{\prime}, E \cup\right.$ $\left.E^{\prime}, \iota \cup \imath^{\prime}\right)$. A connected component of a graph $G$ is a maximal connected subgraph of $G$. The number of connected components of $G$ is denoted $c(G)$. Whenever $V^{\prime} \subseteq V$, the subgraph of $G$ induced by $V^{\prime}$, denoted $G\left[V^{\prime}\right]$, is $\left(V^{\prime}, E_{G}\left(V^{\prime}\right),\left.\iota\right|_{E_{G}\left(V^{\prime}\right)}\right)$. Similarly, whenever $E^{\prime} \subseteq E$, the subgraph of $G$ induced by $E^{\prime}$, denoted $G\left[E^{\prime}\right]$, is $\left(V_{G}\left(E^{\prime}\right), E^{\prime}, l_{E^{\prime}}\right)$.

Definition 2.1.1. An edge-weighting of a tree $\mathrm{T}$ is a map $w: \mathrm{E}(\mathrm{T}) \rightarrow \omega$, and an edge-weighted tree is a pair $(\mathrm{T}, w)$ consisting of a tree $\mathrm{T}$ and an edge-weighting $w$ of $\mathrm{T}$. An edge-weighted tree $(\mathrm{T}, w)$ is finite if $\mathrm{T}$ is finite. Whenever $(T, w)$ is an edge-weighted tree and $k \in \omega$, the subgraph of $T$ induced by $\{e \in E(T) \mid w(e) \geqslant k\}$ is denoted $T_{\geqslant k}$.

We partially order finite edge-weighted trees as in [GGW02].

Definition 2.1.2. Define a binary relation $=_{E}$ on the set $\mathcal{T}$ of finite edgeweighted trees by $(T, w)=E\left(T^{\prime}, w^{\prime}\right)$ if

(1) $e\left(T_{\geqslant l}\right)=e\left(T_{\geqslant l}^{\prime}\right)$ and $c\left(T_{\geqslant l}\right)=c\left(T_{\geqslant l}^{\prime}\right)$ for every $l \in \omega$.

Define a binary relation $<_{E}$ on $\mathcal{T}$ by $(T, w)<_{E}\left(T^{\prime}, w^{\prime}\right)$ if there exists $k \in \omega$ such that

(2) either $e\left(T_{\geqslant k}\right)<e\left(T_{\geqslant k}^{\prime}\right)$ or $e\left(T_{\geqslant k}\right)=e\left(T_{\geqslant k}^{\prime}\right)$ and $c\left(T_{\geqslant k}\right)>c\left(T_{\geqslant k}^{\prime}\right)$, and

(3) $e\left(T_{\geqslant l}\right)=e\left(T_{\geqslant l}^{\prime}\right)$ and $c\left(T_{\geqslant l}\right)=c\left(T_{\geqslant l}^{\prime}\right)$ for every $l \in \mathbb{Z}_{>k}$.

Let $\leqslant \mathrm{E}$ and $\neq_{\mathrm{E}}$ be the binary relations $=_{\mathrm{E}} \cup<_{\mathrm{E}}$ and $(\mathcal{T} \times \mathcal{T})-=_{\mathrm{E}}$ respectively.

Proposition 2.1.3. The binary relation $\leqslant_{\mathrm{E}}$ is a partial order on the set of finite edge-weighted trees.

Proof. Clearly $\leqslant_{\mathrm{E}}$ is reflexive, so we demonstrate that it is antisymmetric and transitive.

Let $(\mathrm{T}, w) \leqslant \mathrm{E}\left(\mathrm{T}^{\prime}, w^{\prime}\right) \leqslant \mathrm{E}(\mathrm{T}, w)$ and suppose that $(\mathrm{T}, w) \neq_{\mathrm{E}}\left(\mathrm{T}^{\prime}, w^{\prime}\right)$. Then as $(T, w)<_{E}\left(T^{\prime}, w^{\prime}\right)$, there exists $k \in \omega$ such that either $e\left(T_{\geqslant k}\right)<$ 
$e\left(T_{\geqslant k}^{\prime}\right)$ or $e\left(T_{\geqslant k}\right)=e\left(T_{\geqslant k}^{\prime}\right)$ and $c\left(T_{\geqslant k}\right)>c\left(T_{\geqslant k}^{\prime}\right)$, and such that $e\left(T_{\geqslant l}\right)=$ $e\left(T_{\geqslant l}^{\prime}\right)$ and $c\left(T_{\geqslant l}\right)=c\left(T_{\geqslant l}^{\prime}\right)$ for every $l \in \mathbb{Z}_{>k}$. Similarly, as $\left(T^{\prime}, w^{\prime}\right)<E$ $(T, w)$, there exists $k^{\prime} \in \omega$ such that either $e\left(T_{\geqslant k^{\prime}}\right)>e\left(T_{\geqslant k^{\prime}}^{\prime}\right)$ or $e\left(T_{\geqslant k^{\prime}}\right)=$ $e\left(T_{\geqslant k^{\prime}}^{\prime}\right)$ and $c\left(T_{\geqslant k^{\prime}}\right)<c\left(T_{\geqslant k^{\prime}}^{\prime}\right)$, and such that $e\left(T_{\geqslant k^{\prime}}\right)=e\left(T_{\geqslant k^{\prime}}^{\prime}\right)$ and $c\left(T_{\geqslant l^{\prime}}\right)=c\left(T_{\geqslant l^{\prime}}^{\prime}\right)$ for every $l^{\prime} \in \mathbb{Z}_{>k^{\prime}}$.

If $k<k^{\prime}$, then as $(T, w)<E\left(T^{\prime}, w^{\prime}\right), e\left(T_{\geqslant k^{\prime}}\right)=e\left(T_{\geqslant k^{\prime}}^{\prime}\right)$ and $c\left(T_{\geqslant k^{\prime}}\right)=$ $c\left(T_{\geqslant k^{\prime}}^{\prime}\right)$, contradicting $\left(T^{\prime}, w^{\prime}\right)<_{E}(T, w)$. Thus $k \nless k^{\prime}$. Similarly, $k^{\prime} \nless k$, so $k=k^{\prime}$. As $e\left(T_{\geqslant k}\right)<e\left(T_{\geqslant k}^{\prime}\right)<e\left(T_{\geqslant k}\right)$ is impossible, $e\left(T_{\geqslant k}\right)=e\left(T_{\geqslant k}^{\prime}\right)$. But then $c\left(T_{\geqslant k}\right)>c\left(T_{\geqslant k}^{\prime}\right)>c\left(T_{\geqslant k}\right)$. Hence $(T, w)=\left(T^{\prime}, w^{\prime}\right)$, and so $\leqslant_{E}$ is antisymmetric.

Now let $(T, w) \leqslant E\left(T^{\prime}, w^{\prime}\right) \leqslant E\left(T^{\prime \prime}, w^{\prime \prime}\right)$ and suppose that $(T, w) \neq E$ $\left(\mathrm{T}^{\prime}, w^{\prime}\right) \neq_{\mathrm{E}}\left(\mathrm{T}^{\prime \prime}, w^{\prime \prime}\right)$. Then as $(\mathrm{T}, w)<_{\mathrm{E}}\left(\mathrm{T}^{\prime}, w^{\prime}\right)$, there exists $k \in \omega$ such that either $e\left(T_{\geqslant k}\right)<e\left(T_{\geqslant k}^{\prime}\right)$ or $e\left(T_{\geqslant k}\right)=e\left(T_{\geqslant k}^{\prime}\right)$ and $c\left(T_{\geqslant k}\right)>c\left(T_{\geqslant k}^{\prime}\right)$, and such that $e\left(T_{\geqslant l}\right)=e\left(T_{\geqslant l}^{\prime}\right)$ and $c\left(T_{\geqslant l}\right)=c\left(T_{\geqslant l}^{\prime}\right)$ for every $l \in \mathbb{Z}_{>k}$. Similarly, as $\left(T^{\prime}, w^{\prime}\right)<E\left(T^{\prime \prime}, w^{\prime \prime}\right)$, there exists $k^{\prime} \in \omega$ such that either $e\left(T_{\geqslant k^{\prime}}^{\prime}\right)<$ $e\left(\mathrm{~T}_{\geqslant k^{\prime}}^{\prime \prime}\right)$ or $e\left(\mathrm{~T}_{\geqslant k^{\prime}}^{\prime}\right)=e\left(\mathrm{~T}_{\geqslant k^{\prime}}^{\prime \prime}\right)$ and $\mathrm{c}\left(\mathrm{T}_{\geqslant k^{\prime}}^{\prime}\right)>\mathrm{c}\left(\mathrm{T}_{\geqslant k^{\prime}}^{\prime \prime}\right)$, and such that $e\left(\mathrm{~T}_{\geqslant k^{\prime}}^{\prime}\right)=$ $e\left(\mathrm{~T}_{\geqslant l^{\prime}}^{\prime \prime}\right)$ and $\mathrm{c}\left(\mathrm{T}_{\geqslant \mathrm{l}^{\prime}}^{\prime}\right)=\mathrm{c}\left(\mathrm{T}_{\geqslant \mathrm{l}^{\prime}}^{\prime \prime}\right)$ for every $\mathrm{l}^{\prime} \in \mathbb{Z}_{>\mathrm{k}^{\prime}}$.

Let $K=\max \left\{k, k^{\prime}\right\}$. Then $e\left(T_{\geqslant L}\right)=e\left(T_{\geqslant L}^{\prime \prime}\right)$ and $c\left(T_{\geqslant L}\right)=c\left(T_{\geqslant L}^{\prime \prime}\right)$ for every $L \in \mathbb{Z}_{>K}$.

Suppose that $k<k^{\prime}$. Then $e\left(T_{\geqslant K}\right)=e\left(T_{\geqslant K}^{\prime}\right)$ and $c\left(T_{\geqslant K}\right)=c\left(T_{\geqslant K}^{\prime}\right)$. If $e\left(T_{\geqslant K}^{\prime}\right)<e\left(T_{\geqslant K}^{\prime \prime}\right)$, then $e\left(T_{\geqslant K}\right)<e\left(T_{\geqslant K}^{\prime \prime}\right)$, so $(T, w)<_{E}\left(T^{\prime \prime}, w^{\prime \prime}\right)$. Otherwise, if $e\left(T_{\geqslant K}^{\prime}\right)=e\left(T_{\geqslant K}^{\prime \prime}\right)$ and $c\left(T_{\geqslant K}^{\prime}\right)>c\left(T_{\geqslant K}^{\prime \prime}\right)$, then $e\left(T_{\geqslant K}\right)=e\left(T_{\geqslant K}^{\prime \prime}\right)$ and $c\left(T_{\geqslant K}\right)>c\left(T_{\geqslant K}^{\prime \prime}\right)$, so $(T, w)<_{E}\left(T^{\prime \prime}, w^{\prime \prime}\right)$.

Now suppose that $k>k^{\prime}$. Then $e\left(T_{\geqslant K}^{\prime}\right)=e\left(T_{\geqslant K}^{\prime \prime}\right)$ and $c\left(T_{\geqslant K}^{\prime}\right)=c\left(T_{\geqslant K}^{\prime \prime}\right)$. If $e\left(T_{\geqslant K}\right)<e\left(T_{\geqslant K}^{\prime}\right)$, then $e\left(T_{\geqslant K}\right)<e\left(T_{\geqslant K}^{\prime \prime}\right)$, so $(T, w)<E\left(T^{\prime \prime}, w^{\prime \prime}\right)$. Otherwise, if $e\left(T_{\geqslant K}\right)=e\left(T_{\geqslant K}^{\prime}\right)$ and $c\left(T_{\geqslant K}\right)>c\left(T_{\geqslant K}^{\prime}\right)$, then $e\left(T_{\geqslant K}\right)=e\left(T_{\geqslant K}^{\prime \prime}\right)$ and $c\left(T_{\geqslant K}\right)>c\left(T_{\geqslant K}^{\prime \prime}\right)$, so $(T, w)<E\left(T^{\prime \prime}, w^{\prime \prime}\right)$.

Lastly, suppose that $k=k^{\prime}$. If either $e\left(T_{\geqslant K}\right)<e\left(T_{\geqslant K}^{\prime}\right)$ or $e\left(T_{\geqslant K}^{\prime}\right)<$ $e\left(T_{\geqslant K}^{\prime \prime}\right)$, then $e\left(T_{\geqslant K}\right)<e\left(T_{\geqslant K}^{\prime \prime}\right)$, in which case $(T, w)<_{E}\left(T^{\prime \prime}, w^{\prime \prime}\right)$. Otherwise, $e\left(T_{\geqslant K}\right)=e\left(T_{\geqslant K}^{\prime}\right)=e\left(T_{\geqslant K}^{\prime \prime}\right)$ and $c\left(T_{\geqslant K}\right)>c\left(T_{\geqslant K}^{\prime}\right)>c\left(T_{\geqslant K}^{\prime \prime}\right)$, so $e\left(T_{\geqslant K}\right)=e\left(T_{\geqslant K}^{\prime \prime}\right)$ and $c\left(T_{\geqslant K}\right)>c\left(T_{\geqslant K}^{\prime \prime}\right)$, in which case $(T, w)<_{E}\left(T^{\prime \prime}, w^{\prime \prime}\right)$. 
Hence $\leqslant_{E}$ is transitive.

\subsection{Linear Algebra}

This section reviews some elementary properties of linear subspaces and furnishes a pair of technical lemmas for later use. We take the dimension of a vector space to be (symbolically) $\infty$ if it is not finite. The set of subspaces of a vector space $V$ is denoted $\mathcal{S}(V)$. It is well-known that $(\mathcal{S}(\mathrm{V}),+, \cap)$ is a modular lattice for every vector space $\mathrm{V}$.

Modular Law. Let $\mathrm{V}$ be a vector space and let $\mathrm{U}^{\prime}, \mathrm{U}, \mathrm{W} \leqslant \mathrm{V}$ be such that $\mathrm{U}^{\prime} \subseteq \mathrm{u}$. Then $\mathrm{U} \cap\left(\mathrm{U}^{\prime}+\mathrm{W}\right)=\mathrm{U}^{\prime}+(\mathrm{u} \cap \mathrm{W})$

While $(\mathcal{S}(\mathrm{V}),+, \cap)$ is modular for every vector space $V$, it is not always distributive (consider any vector space having dimension at least 2 ).

The following technical lemma is used in Section 6.3.

Lemma 2.2.1. Let $\mathrm{V}$ be a vector space, let $\mathrm{k} \in \mathrm{\omega}$ and let $\mathrm{U}, \mathrm{U}^{\prime} \leqslant \mathrm{V}$ each have dimension at least $\mathrm{k}$. Then there exists a sequence $\mathrm{U}=\mathrm{U}_{1}, \mathrm{U}_{2}, \ldots, \mathrm{U}_{\mathrm{n}}=\mathrm{U}^{\prime}$ of subspaces of $\mathrm{V}$ such that

(1) $\operatorname{dim}\left(\mathrm{U}_{i} \cap \mathrm{U}_{i+1}\right) \geqslant k$ for each $i=1, \ldots, n-1$; and

(2) $\operatorname{dim}\left(u_{i}\right)=k+1$ for each $i=2, \ldots, n-1$.

Proof. If $\mathrm{U}=\mathrm{U}^{\prime}$, simply take $\mathrm{n}=1$ and set $\mathrm{U}_{1}=\mathrm{U}$. Otherwise, choose $W \leqslant U$ and $W^{\prime} \leqslant U^{\prime}$ each having dimension $k$ and let $\left\{b_{1}, \ldots, b_{j}\right\}$ be a basis for $W \cap W^{\prime}$. Extend this by $c_{j+1}, \ldots, c_{k}$ to a basis for $W$ and (independently) by $d_{j+1}, \ldots, d_{k}$ to a basis for $W^{\prime}$. Let

$$
\begin{aligned}
\mathrm{u}_{1} & =\mathrm{u}, \\
\mathrm{u}_{2} & =\left\langle\mathrm{b}_{1}, \ldots, \mathrm{b}_{j}, \mathrm{c}_{j+1}, \ldots, \mathrm{c}_{k}, \mathrm{~d}_{j+1}\right\rangle, \\
\mathrm{u}_{3} & =\left\langle\mathrm{b}_{1}, \ldots, \mathrm{b}_{j}, \mathrm{c}_{j+1}, \ldots, \mathrm{c}_{k-1}, \mathrm{~d}_{j+1}, \mathrm{~d}_{j+2}\right\rangle,
\end{aligned}
$$




$$
\begin{aligned}
u_{k-j+1} & =\left\langle b_{1}, \ldots, b_{j}, c_{j+1}, d_{j+1}, \ldots, d_{k}\right\rangle, \\
u_{k-j+2} & =u^{\prime} .
\end{aligned}
$$

Then clearly the resulting sequence $U_{1}, \ldots, U_{k-j+2}$ satisfies (1) and (2).

We now consider complements and projections.

Definition 2.2.2. Let $\mathrm{V}$ be a vector space and let $\mathrm{W} \leqslant \mathrm{V}$. Then $\mathrm{U} \leqslant \mathrm{V}$ is a complementary subspace or complement of $\mathrm{W}$ in $\mathrm{V}$ if $\mathrm{V}=\mathrm{W} \oplus \mathrm{U}$.

Proposition 2.2.3. Every subspace of a vector space $\mathrm{V}$ has a complement in $\mathrm{V}$.

For completeness, we reproduce a standard argument that may be found on p. 26 of [Gre75].

Proof. Let $\mathrm{V}$ be a vector space and let $\mathrm{W} \leqslant \mathrm{V}$. Without loss of generality, $\{\boldsymbol{0}\} \neq \mathrm{W} \neq \mathrm{V}$, for otherwise the result is trivial. Let $\mathrm{B}$ be a basis for $W$. Extend this by $B^{\prime}$ to a basis for $V$ and set $U=\left\langle B^{\prime}\right\rangle$. Clearly $V=$ $W+U$. Let $v \in W \cap U$. Then $v$ is both a linear combination of elements of $B$ and a linear combination of elements of $B^{\prime}$. The difference of these linear combinations is $\mathbf{0}$, so as $\mathrm{B} \sqcup \mathrm{B}^{\prime}$ is linearly independent, both linear combinations are $\mathbf{0}$. Hence $v=\mathbf{0}$. The result follows.

Definition 2.2.4. Let $\mathrm{V}=\mathrm{W} \oplus \mathrm{U}$ be a vector space. Then the projection of $\mathrm{V}$ onto $\mathrm{U}$ along $\mathrm{W}$ is the map $\pi: \mathrm{V} \rightarrow \mathrm{V}$ defined by

$$
\pi(w+u)=u
$$

for every $w \in W$ and every $u \in U$.

The next proposition lists some elementary properties of projections.

Proposition 2.2.5. Let $\mathrm{V}=\mathrm{W} \oplus \mathrm{U}$ be a vector space and let $\pi$ be the projection of $\mathrm{V}$ onto $\mathrm{U}$ along $\mathrm{W}$. Then 
(1) $\pi$ is an endomorphism of $\mathrm{V}$,

(2) $\operatorname{ker}(\pi)=W$,

(3) $\operatorname{im}(\pi)=\mathrm{U}$,

(4) $\pi\left[\mathrm{V}^{\prime}\right]=\mathrm{U} \cap\left(\mathrm{W}+\mathrm{V}^{\prime}\right) \leqslant \mathrm{V}$ for every $\mathrm{V}^{\prime} \leqslant \mathrm{V}$, and

(5) $\pi^{2}=\pi$.

Clearly complements are not always unique. However, they are always isomorphic.

Proposition 2.2.6. Let $\mathrm{V}$ be a vector space and let $\mathrm{W} \leqslant \mathrm{V}$. Then all complements of $\mathrm{W}$ in $\mathrm{V}$ are isomorphic.

We reproduce a standard argument sketched on pp. 79-80 of [Rom05].

Proof. Let $\mathrm{U}_{1}$ and $\mathrm{U}_{2}$ be complements of $\mathrm{W}$ in $\mathrm{V}$. For each $i$, let $\pi_{\mathrm{i}}$ be the projection of $V$ onto $U_{i}$ along $W$ and define $\pi_{i}^{\prime}: v / w \rightarrow U_{i}$ by

$$
\pi_{\mathfrak{i}}^{\prime}(v+W)=\pi_{i}(v)
$$

for every $v+W \in \mathrm{V} / \mathrm{w}$. Then by the First Isomorphism Theorem,

$$
\mathrm{v} / \mathrm{w} \cong \mathrm{im}\left(\pi_{\mathrm{i}}\right)=\mathrm{U}_{\mathrm{i}}
$$

for each $i$. The result follows.

We close this section with another technical lemma.

Lemma 2.2.7. Let $\mathrm{V}, \mathrm{V}^{\prime}$ be subspaces of a vector space and let $\mathrm{V}_{1} \leqslant \mathrm{~V}, \mathrm{~V}_{2} \leqslant$ $\mathrm{V} \cap \mathrm{V}^{\prime}$ be such that $\mathrm{V}_{1} \cap \mathrm{V}^{\prime} \subseteq \mathrm{V}_{2}$ and $\operatorname{dim}\left(\mathrm{V}_{1}\right) \leqslant \operatorname{dim}\left(\mathrm{V}_{2}\right) \in \omega$. Then there exist finite linearly independent $\mathrm{X} \subseteq\left(\mathrm{V}_{1}+\mathrm{V}_{2}\right)-\left(\mathrm{V}_{1} \cup \mathrm{V}^{\prime}\right)$ and a complement $\mathrm{U} \geqslant \mathrm{V}^{\prime}$ of $\langle\mathrm{X}\rangle$ in $\mathrm{V}+\mathrm{V}^{\prime}$ such that $\pi\left[\mathrm{V}_{1}\right] \leqslant \mathrm{V}_{2}$ and $\operatorname{dim}\left(\pi\left[\mathrm{V}_{1}\right]\right)=\operatorname{dim}\left(\mathrm{V}_{1}\right)$, where $\pi$ is projection of $\mathrm{V}+\mathrm{V}^{\prime}$ onto $\mathrm{U}$ along $\langle\mathrm{X}\rangle$. 


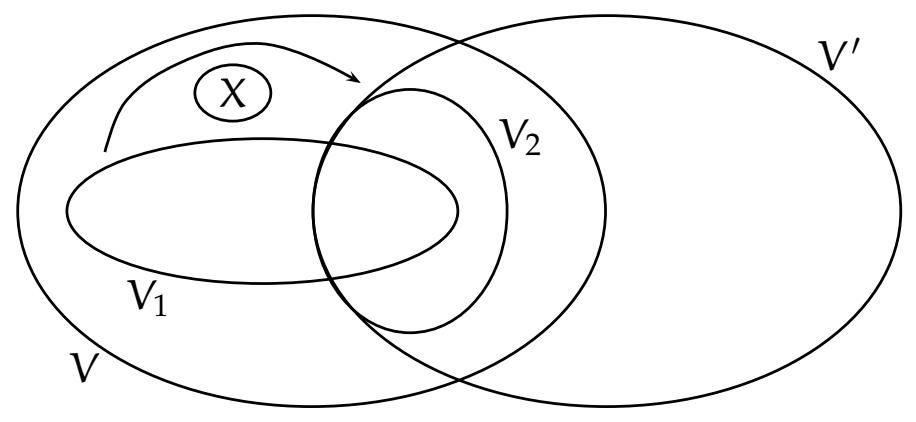

Figure 2.1: Proof of Lemma 2.2.7.

Proof. It may be helpful to consider Figure 2.1.

Let $V_{i}^{\prime}$ be a complement of $V_{1} \cap V_{2}$ in $V_{i}$ for each $i$. As $\operatorname{dim}\left(V_{1}\right) \leqslant$ $\operatorname{dim}\left(V_{2}\right) \in \omega$, necessarily $\operatorname{dim}\left(V_{1}^{\prime}\right) \leqslant \operatorname{dim}\left(V_{2}^{\prime}\right)$. Let $\left\{y_{1}, \ldots, y_{n}\right\}$ be a basis for $V_{1}^{\prime}$, let $\left\{z_{1}, \ldots, z_{n}\right\} \subseteq V_{2}^{\prime}$ be linearly independent and let $X=\left\{y_{1}-\right.$ $\left.z_{1}, \ldots, y_{n}-z_{n}\right\}$. Clearly $X \subseteq\left(V_{1}+V_{2}\right)-\left(V_{1} \cup V^{\prime}\right)$ is linearly independent. Let $\mathrm{U} \geqslant \mathrm{V}^{\prime}$ be a complement of $\langle\mathrm{X}\rangle$ in $\mathrm{V}+\mathrm{V}^{\prime}$ and let $\pi$ be projection of $\mathrm{V}+\mathrm{V}^{\prime}$ onto $\mathrm{U}$ along $\langle\mathrm{X}\rangle$. Then $\pi\left[\mathrm{V}_{1}\right]=\left(\mathrm{V}_{1} \cap \mathrm{V}_{2}\right) \oplus\left\langle z_{1}, \ldots, z_{\mathrm{n}}\right\rangle \leqslant \mathrm{V}_{2}$, from which it follows that $\operatorname{dim}\left(\pi\left[\mathrm{V}_{1}\right]\right)=\operatorname{dim}\left(\mathrm{V}_{1}\right)$.

\subsection{Rado's Selection Lemma}

We shall later utilise the Axiom of Choice, as well as the equivalent lemmas of Zorn and Rado, each of which is stated below. We omit the wellknown derivation of Zorn's Lemma from the Axiom of Choice, but include Rado's brief (and accessible) derivation of the possibly less-familiar Selection Lemma.

Definition 2.3.1. Let $X$ and I be sets and let $X=\left\{X_{i} \mid i \in I\right\}$ be a collection of non-empty subsets of $X$. Then a choice function for $X$ is a function $f: I \rightarrow X$ such that $f(i) \in X_{i}$ for every $i \in I$. 
Axiom of Choice. Every collection of non-empty sets has a choice function.

Zorn's Lemma. Let $(\mathrm{X}, \leqslant)$ be a non-empty poset such that every chain in $\mathrm{X}$ has an upper bound in $\mathrm{X}$. Then $\mathrm{X}$ contains a maximal element.

Rado's Selection Lemma. Let $X$ and $I$ be sets and let $X=\left\{X_{i} \mid i \in I\right\}$ be a collection of finite non-empty subsets of $\mathrm{X}$. For every finite $\mathrm{J} \subseteq \mathrm{I}$, let $\mathrm{f}_{\mathrm{J}}$ be a choice function for $\left\{X_{j} \mid j \in J\right\}$. Then there exists a choice function $f$ for $X$ such that whenever $\mathrm{J} \subseteq \mathrm{I}$ is finite there exists finite $\mathrm{K} \subseteq \mathrm{I}$ containing J for which $\left.f\right|_{J}=\left.f_{K}\right|_{J}$.

We reproduce the argument given in [Rad71], with some additional details included.

Proof. If $I$ is finite, simply set $f=f_{I}$. So suppose that $I$ is infinite. Let $\Omega$ be the set of all collections $Z=\left\{Z_{i} \mid i \in I\right\}$ such that

- $Z_{i} \subseteq X_{i}$ for every $i \in I$, and

- whenever $\mathrm{J} \subseteq \mathrm{I}$ is finite, there exists finite $\mathrm{K} \subseteq \mathrm{I}$ containing $\mathrm{J}$ such that $f_{K}(j) \in Z_{j}$ for every $j \in J$.

Then $X \in \Omega$ by hypothesis. Define $y=\left\{Y_{i} \mid i \in I\right\} \in \Omega$ as follows. If I is countable, write $I=\omega$. Then, using the finiteness of each $X_{i}$, choose a minimal $Y_{0} \subseteq X_{0}$ such that $\left\{Y_{0}, X_{1}, X_{2}, \ldots\right\} \in \Omega$, then a minimal $Y_{1} \subseteq X_{1}$ such that $\left\{Y_{0}, Y_{1}, X_{2}, \ldots\right\} \in \Omega$, and so forth.

Otherwise, define a partial order $\leqslant$ on $\Omega$ by $Z=\left\{Z_{i} \mid i \in I\right\} \leqslant Z^{\prime}=$ $\left\{Z_{i}^{\prime} \mid i \in I\right\}$ if and only if $Z_{i} \supseteq Z_{i}^{\prime}$ for every $i \in I$. Let $\Xi=\left\{Z^{\ell} \mid \ell \in L\right\}$ be a non-empty chain in $\Omega$ with $Z^{\ell}=\left\{Z_{i}^{\ell} \mid i \in I\right\}$ for every $\ell \in L$, and let $z=\left\{Z_{i} \mid i \in I\right\}$, where $Z_{i}=\bigcap_{\ell \in L} Z_{i}^{\ell}$ for every $i \in I$. Then clearly $Z_{i} \subseteq X_{i}$ for every $i \in I$ and $z$ is an upper bound for $\Xi$. Let $J \subseteq I$ be finite. If $J$ is empty, then $f_{J}$ vacuously has $f_{J}(j) \in Z_{j}$ for every $j \in J$. Otherwise, as each $Z_{j}^{\ell}$ is finite, for each $j \in J$ there exists $\ell_{j} \in L$ such that $Z_{j}^{\ell_{j}}=Z_{j}$. So as $\left\{Z^{\ell_{j}} \mid j \in J\right\}$ is a chain, there exists $\ell^{\prime} \in L$ such that $Z_{j}^{\ell^{\prime}}=Z_{j}$ for every $j \in J$. 
Then $Z^{\ell^{\prime}} \in \Omega$ implies that there exists finite $K \subseteq$ I containing $J$ such that $f_{K}(j) \in Z_{j}$ for every $j \in J$. Thus $z \in \Omega$, and so $\Omega$ has a maximal element $y$ by Zorn's Lemma.

In either case, $P:=\left\{(i, y) \mid i \in I, y \in Y_{i}\right\}$ is minimal. Fix $i^{\prime} \in I$. Then $Y_{i^{\prime}}$ is non-empty by the definition of $\Omega$, so let $y \in Y_{i^{\prime}}$. Then by the minimality of $P$, there exists finite $J_{y} \subseteq$ I containing $i^{\prime}$ such that $f_{K}\left(i^{\prime}\right)=y$ whenever $K \subseteq I$ is finite, contains $J_{y}$ and $f_{K}(j) \in Y_{j}$ for every $j \in J_{y}$. Now let $y, y^{\prime} \in Y_{i^{\prime}}$. As $J^{\prime}:=J_{y} \cup J_{y^{\prime}}$ is a finite subset of $I$, it follows from the definition of $\Omega$ that there exists finite $\mathrm{K}^{\prime} \subseteq$ I containing $\mathrm{J}^{\prime}$ such that $f_{K^{\prime}}(j) \in Y_{j}$ for every $j \in J$. It follows from the definitions of $J_{y}$ and $J_{y^{\prime}}$ that $y=f_{K^{\prime}}\left(i^{\prime}\right)=y^{\prime}$. Hence $\left|Y_{i^{\prime}}\right|=1$, so define $f$ by setting $Y_{i}=\{f(i)\}$ for every $i \in I$.

Finally, let $\mathrm{J} \subseteq \mathrm{I}$ be finite. Then there exists finite $\mathrm{K} \subseteq$ I containing J such that $f_{K}(j) \in Y_{j}$ for every $j \in J$, that is, $\left.f\right|_{J}=\left.f_{K}\right|_{J}$. 


\section{Chapter 3}

\section{Independence Spaces}

This thesis is primarily concerned with point configurations, which may be thought of as "infinite represented matroids." These are introduced explicitly in Chapter 4. Many results obtainable for configurations are purely matroidal, and thus most naturally formulated and proven matroidally. This chapter develops the matroid theory necessary for the formulation and proof of such results. Despite the treatment of infinite structures, the material covered remains very similar to basic matroid theory as found in [Ox104].

\subsection{Definitions}

Definition 3.1.1. A pre-independence space is a pair $M=(E, \mathcal{J})$ consisting of a set $E$ and a collection $\mathcal{J}$ of subsets of $E$ satisfying:

(I1) $\varnothing \in \mathcal{J}$.

(I2) $\mathrm{I}_{1} \in \mathcal{J}$ whenever $\mathrm{I}_{1} \subseteq \mathrm{I}_{2} \in \mathcal{J}$.

(I3) (Finite Augmentation) Whenever $\mathrm{I}_{1}, \mathrm{I}_{2} \in \mathcal{J}$ are such that $\left|\mathrm{I}_{1}\right|<\left|\mathrm{I}_{2}\right| \in \omega$, there exists $e \in \mathrm{I}_{2}-\mathrm{I}_{1}$ for which $\mathrm{I}_{1} \sqcup\{e\} \in \mathcal{J}$. 
The ground set of $M$, denoted $E(M)$, is $E$, and $M$ is a pre-independence space on $\mathrm{E}$. A subset $\mathrm{X} \subseteq \mathrm{E}$ is dependent if $\mathrm{X} \notin \mathrm{J}$ and independent otherwise. A circuit is a minimal dependent set, while a basis is a maximal independent set. A loop is an element $e \in E$ for which $\{e\}$ is dependent, a parallel pair is a 2-element circuit and a triangle is a 3-element circuit. Note that loops are not circuits. The collections of independent sets, circuits, bases and loops of $M$ are denoted by $\mathcal{J}(M), \mathcal{C}(M), \mathcal{B}(M)$ and $\mathcal{L}(M)$ respectively. A pre-independence space is simple if it has neither loops nor parallel pairs.

An independence space or finitary matroid is a pre-independence space $(E, \mathcal{J})$ that also satisfies:

(I4) (Finite Character) $X \in \mathcal{J}$ whenever $X \subseteq E$ has $Y \in \mathcal{J}$ for every finite $\mathrm{Y} \subseteq \mathrm{X}$

A matroid is a pre-independence space having a finite ground set.

It is immediate that every matroid is also an independence space, and that every dependent set in an independence space contains a circuit, which is necessarily finite.

To date, abstract treatments of independence have been based either on universal algebra or on set theory (see p. 112 of [Gła03]). The algebraic approach rose to prominence with the introduction of " $v^{*}$-algebras" by Marczewski in [Mar58]. This article marked the beginning of an extensive algebraic investigation of independence led by Polish researchers during the 1960s. Chapter 5 of [Grä79] provides an introduction to this area, while a survey and comprehensive bibliography is given in [Gła79]. A number of infinite matroid results follow from this theory, for independence spaces can be axiomatised by closure operators (see Section 3.5), which can in turn be obtained as the algebraic closure operators of universal algebras (see p. 300 of [BF48]).

The set-theoretic approach to independence encompasses matroids, which were first introduced by Whitney in the seminal article [Whi35]. 
Generalisations of matroids to infinite settings do not appear to be wellrepresented in the literature until the late 1960s onwards. Earlier work includes Rado's treatment of the "independence relations" introduced in [Rad42], which were subsequently investigated by both Lazarson and Ingleton.

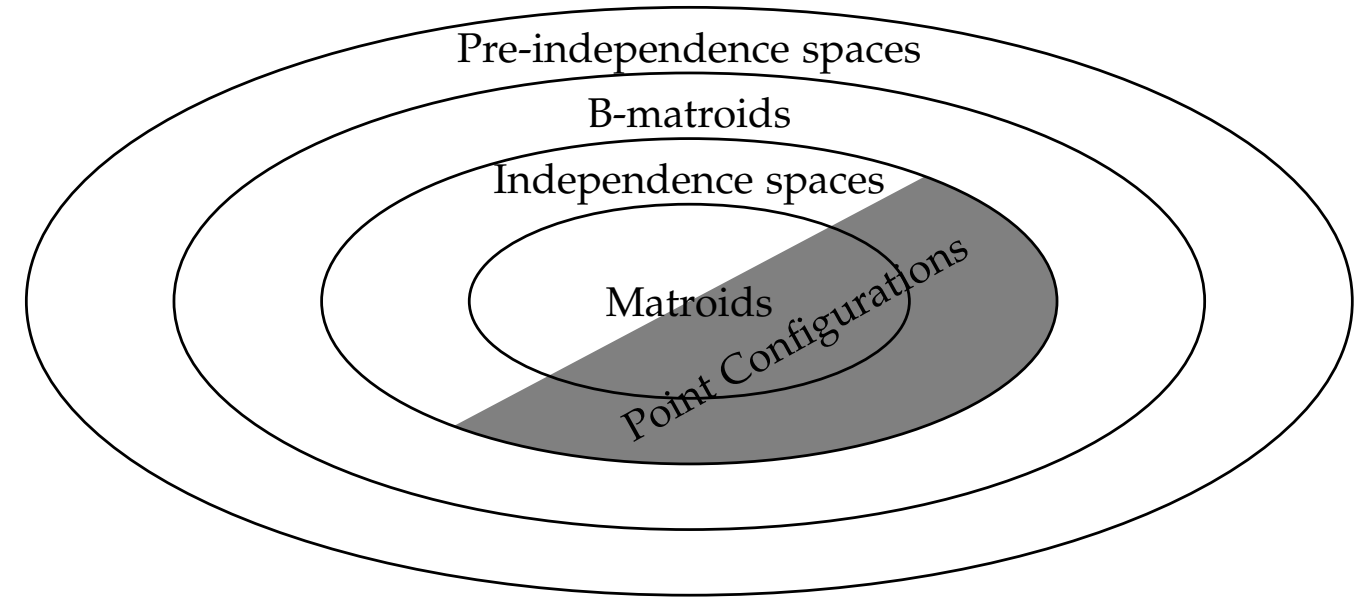

Figure 3.1: Some classes of matroidal objects.

The simplest extension of matroids to infinite sets consists of allowing infinite ground sets. This yields the pre-independence spaces. These have received limited attention in their own right, as their weak structure fails to capture much of the behaviour that makes matroids interesting. For example, a pre-independence space may have neither circuits nor bases (see p. 74 of [Ox192]).

Such weaknesses can be avoided by "determining global structure locally" through the imposition of (I4). This yields the independence spaces. These are the focus of the remainder of this chapter, for they are the appropriate objects for capturing the matroidal structure of point configurations (see pp. 50-51). Nonetheless, (I4) is a very strong requirement. Its imposition prevents the development of a reasonable theory of duality 
analogous to that of matroids.

In order to have a satisfactory theory of duality, it is necessary to work with the "B-matroids" introduced by Higgs in [Hig69]. These may possess infinite dependencies, suggesting that topology (perhaps in the form of topological graph theory) may be necessary for the development of an effective representation theory for these objects. This appears to be a topic of current and growing interest.

Identifying point configurations with the independence spaces that they represent, the relationships between these classes of objects are summarised in Figure 3.1.

Denote the power set of a set $E$ by $\mathcal{P}(E)$. We close this section by introducing a class of independence spaces that shall prove revealing later.

Proposition 3.1.2. Let $\mathrm{G}$ be a graph having edge set $\mathrm{E}$. Define $\mathcal{J} \subseteq \mathcal{P}(\mathrm{E})$ by $X \in \mathcal{J}$ if and only if no subset of $X$ is the edge set of a cycle of $G$. Then $(E, \mathcal{J})$ is an independence space.

We omit the straightforward proof of this result, which is given for finite graphs on p. 11 of [Ox104] (see also p. 75 of [Ox192]).

Definition 3.1.3. Let $G$ be a graph. Then the cycle independence space of $G$, denoted $M(G)$, is the independence space derived from $G$ via Proposition 3.1.2. An independence space $M$ is graphic if $M=M(G)$ for some graph G.

Note that for every graph $\mathrm{G}$,

- the circuits of $M(G)$ are precisely the edge sets of the cycles of $G$, and

- the bases of $M(G)$ are precisely the edge sets of the spanning forests of G. 


\subsection{Circuits}

The collection of circuits of an independence space satisfies the properties listed in the next proposition.

Proposition 3.2.1. Let $M=(E, J)$ be an independence space with collection of circuits C. Then:

(C1) $\varnothing \notin \mathcal{C}$.

(C2) $\mathcal{C}$ is an antichain under set inclusion.

(C3) (Weak Circuit Elimination) Whenever $\mathrm{C}_{1}, \mathrm{C}_{2} \in \mathcal{C}$ are distinct and $\mathrm{e} \in \mathrm{E}$, there exists $\mathrm{C}_{3} \in \mathcal{C}$ such that $\mathrm{C}_{3} \subseteq\left(\mathrm{C}_{1} \cup \mathrm{C}_{2}\right)-\{e\}$.

(C4) Every $\mathrm{C} \in \mathrm{C}$ is finite.

We reproduce the argument given on p. 9 of [Ox104].

Proof. Statement (C1) follows from (I1), while (C2) is a consequence of the minimality of circuits. Statement (C4) follows from (C2) and (I4) forcing every infinite dependent set to contain a finite dependent set. So we demonstrate (C3): let $C_{1}, C_{2} \in \mathcal{C}$ be distinct and let $e \in E$. The result is immediate if $e \notin C_{1} \cap C_{2}$, so suppose that $e \in C_{1} \cap C_{2}$ and that $\left(C_{1} \cup C_{2}\right)$ $\{e\}$ contains no circuit. Then $\left(C_{1} \cup C_{2}\right)-\{e\} \in \mathcal{J}$. It follows from (C2) that $C_{2}-C_{1}$ is non-empty, so let $f \in C_{2}-C_{1}$. Then $C_{2}-\{f\} \in \mathcal{J}$ also. Let I be a maximal independent subset of $C_{1} \cup C_{2}$ containing $C_{2}-\{f\}$. Necessarily $\mathrm{f} \notin \mathrm{I}$. As $\mathrm{C}_{1} \in \mathcal{C}$, there exists $\mathrm{g} \in \mathrm{C}_{1}$ such that $\mathrm{g} \notin \mathrm{I}$. So as $\mathrm{g} \neq \mathrm{f}$,

$$
|\mathrm{I}| \leqslant\left|\left(C_{1} \cup \mathrm{C}_{2}\right)-\{\mathrm{f}, \mathrm{g}\}\right|=\left|\mathrm{C}_{1} \cup \mathrm{C}_{2}\right|-2<\left|\left(\mathrm{C}_{1} \cup \mathrm{C}_{2}\right)-\{e\}\right|
$$

But then (I3) implies that there exists $h \in\left(C_{1} \cup C_{2}\right)-(I \cup\{e\})$ such that $\mathrm{I} \sqcup\{\mathrm{h}\} \in \mathcal{J}$, contradicting the choice of I. Hence (C3) holds.

In fact, $(\mathrm{C} 1)-(\mathrm{C} 4)$ provide an alternative axiomatisation of independence spaces. A proof of this (for matroids) may be found on p. 10 of [Ox104]. 
Weak Circuit Elimination may be strengthened, as shown in the next proposition.

Proposition 3.2.2. (C1)-(C3) are equivalent to (C1), (C2) and:

(C3') (Strong Circuit Elimination) Whenever $C_{1}, C_{2} \in \mathcal{C}, e \in C_{1} \cap C_{2}$ and $\mathrm{f} \in \mathrm{C}_{1}-\mathrm{C}_{2}$, there exists $\mathrm{C}_{3} \in \mathcal{C}$ such that $\mathrm{f} \in \mathrm{C}_{3} \subseteq\left(\mathrm{C}_{1} \cup \mathrm{C}_{2}\right)-\{\mathrm{e}\}$.

Proof. It suffices to consider the forward implication. Aiming for a contradiction, suppose that $\left(\mathrm{C}^{\prime}\right)$ fails for some $\left(C_{1}, C_{2}, e, f\right)$. Without loss of generality, $\left|C_{1} \cup C_{2}\right|$ is minimal. By (C3), there exists $C_{3} \in \mathcal{C}$ such that $C_{3} \subseteq$ $\left(C_{1} \cup C_{2}\right)-\{e\}$. By hypothesis, $f \notin C_{3}$, and necessarily $e \in C_{2}-C_{3}$. As $\mathrm{C}_{3} \nsubseteq \mathrm{C}_{1}$, there exists $\mathrm{g} \in \mathrm{C}_{3} \cap\left(\mathrm{C}_{2}-\mathrm{C}_{1}\right)$. So as $\mathrm{C}_{2} \cup \mathrm{C}_{3} \subseteq\left(\mathrm{C}_{1} \cup \mathrm{C}_{2}\right)-\{\mathrm{f}\}$, $\left|C_{2} \cup C_{3}\right|<\left|C_{1} \cup C_{2}\right|$. Thus $\left(C^{\prime}\right)$ holds for $\left(C_{2}, C_{3}, g, e\right)$.

Consequently there exists $C_{4} \in \mathcal{C}$ such that $e \in C_{4} \subseteq\left(C_{2} \cup C_{3}\right)-\{g\}$. Now, $f \in C_{1}-C_{4}$ and $e \in C_{1} \cap C_{4}$. As $C_{1} \cup C_{4} \subseteq\left(C_{1} \cup C_{2}\right)-\{g\}, \mid C_{1} \cup$ $\mathrm{C}_{4}|<| \mathrm{C}_{1} \cup \mathrm{C}_{2} \mid$. So $\left(\mathrm{C}^{\prime}\right)$ holds for $\left(\mathrm{C}_{1}, \mathrm{C}_{4}, e, f\right)$.

But then there exists $C_{5} \in \mathcal{C}$ such that $f \in C_{5} \subseteq\left(C_{1} \cup C_{4}\right)-\{e\} \subseteq\left(C_{1} \cup\right.$ $\left.C_{2}\right)-\{e\}$, contradicting the choice of $\left(C_{1}, C_{2}, e, f\right)$. Hence $\left(C 3^{\prime}\right)$ holds.

Proposition 3.2.3. Let $\mathrm{M}=(\mathrm{E}, \mathrm{J})$ be an independence space, let $\mathrm{I} \in \mathcal{J}$ and let $e \in \mathrm{E}-\mathrm{I}$ be such that $\mathrm{I} \sqcup\{\mathrm{e}\} \notin \mathcal{J}$. Then there exists a unique $\mathrm{C} \in \mathrm{C}(\mathrm{M})$ for which $e \in C \subseteq \mathrm{I} \sqcup\{e\}$.

Proof. Let $X=\{C \in \mathcal{C}(M) \mid e \in C \subseteq I \sqcup\{e\}\}$. Then $X$ is non-empty by (I4), so let $C, C^{\prime} \in X$. If $C \neq C^{\prime}$, then Weak Circuit Elimination asserts the existence of $C^{\prime \prime} \in \mathcal{C}(M)$ such that $C^{\prime \prime} \subseteq\left(C \cup C^{\prime}\right)-\{e\} \subseteq I$, which is impossible. Hence $C=C^{\prime}$, establishing the result.

This result motivates the following definition.

Definition 3.2.4. Let $M=(E, \mathcal{J})$ be an independence space, let $B \in \mathcal{B}(M)$ and let $e \in E-B$. Then the fundamental circuit of $e$ with respect to $B$, denoted $\mathrm{C}(e, \mathrm{~B})$, is the unique $\mathrm{C} \in \mathcal{C}(M)$ such that $e \in \mathrm{C} \subseteq \mathrm{B} \sqcup\{e\}$. 


\subsection{Bases and Restriction}

The next result is well known in the vector space context.

Proposition 3.3.1. Let $\mathrm{M}=(\mathrm{E}, \mathrm{J})$ be an independence space and let $\mathrm{I} \subseteq \mathrm{X} \subseteq$ $\mathrm{E}$ be such that $\mathrm{I} \in \mathrm{J}$. Then there exists a maximal independent subset of $\mathrm{X}$ containing I. In particular, every independent set is contained in a basis.

Proof. Let $X=\{\mathrm{J} \in \mathcal{J} \mid \mathrm{I} \subseteq \mathrm{J} \subseteq \mathrm{X}\}$ be partially ordered by set inclusion. Let $y$ be a chain in $X$ and set $B=\bigcup y$. Suppose that $\mathrm{B} \notin X$. As $\mathrm{I} \subseteq \mathrm{B} \subseteq \mathrm{X}, \mathrm{B} \notin$ J. So $B$ contains a dependent subset $\left\{b_{1}, \ldots, b_{n}\right\}$ by (I4). For each $i$ there exists $B_{i} \in y$ containing $b_{i}$. As $\left\{B_{1}, \ldots, B_{n}\right\}$ is a chain, some $B_{j}$ contains each $B_{i}$. In particular, $\left\{b_{1}, \ldots, b_{n}\right\} \subseteq B_{j}$, contradicting the independence of $B_{j}$. Hence $B \in X$ is an upper bound for $y$. It follows from Zorn's Lemma that $X$ contains a maximal element, yielding the result.

Definition 3.3.2. Let $M=(E, \mathcal{J})$ be an independence space and let $X \subseteq E$. The restriction of $M$ to $X$, denoted $M \mid X$, is $(X,\{Y \subseteq X \mid Y \in \mathcal{J}\})$. The deletion of $X$ from $M$, denoted $M \backslash X$, is $M \mid(E-X)$.

Observing that (I1)-(I4) are each preserved by restriction gives the following proposition.

Proposition 3.3.3. Let $M$ be an independence space and let $X \subseteq E(M)$. Then $\mathrm{M} \mid \mathrm{X}$ and $\mathrm{M} \backslash \mathrm{X}$ are independence spaces.

Proposition 3.3.4. Let $M=(E, J)$ be an independence space with collection of bases $\mathcal{B}$. Then:

(B1) $\mathcal{B}$ is non-empty.

(B2) $\mathcal{B}$ is an antichain under set inclusion.

(B3) (Middle Basis) Whenever $\mathrm{X} \subseteq \mathrm{Y} \subseteq \mathrm{E}$ and $\mathrm{B}_{1}, \mathrm{~B}_{2} \in \mathcal{B}$ are such that $\mathrm{X} \subseteq \mathrm{B}_{1}$ and $\mathrm{B}_{2} \subseteq \mathrm{Y}$, there exists $\mathrm{B}_{3} \in \mathcal{B}$ such that $\mathrm{X} \subseteq \mathrm{B}_{3} \subseteq \mathrm{Y}$. 
(B4) Whenever $\mathrm{X} \subseteq \mathrm{E}$ is not contained in any $\mathrm{B} \in \mathcal{B}$, some finite $\mathrm{Y} \subseteq \mathrm{X}$ is not contained in any $\mathrm{B} \in \mathcal{B}$.

Proof. Statement (B1) is a consequence of (I1) and Proposition 3.3.1, while (B2) is a consequence of the maximality of bases. Statement (B4) follows easily from (I4) and Proposition 3.3.1. So we show Middle Basis: let $\mathrm{X} \subseteq \mathrm{Y} \subseteq \mathrm{E}$ and let $\mathrm{B}_{1}, \mathrm{~B}_{2} \in \mathcal{B}$ be such that $\mathrm{X} \subseteq \mathrm{B}_{1}$ and $\mathrm{B}_{2} \subseteq \mathrm{Y}$. By Proposition 3.3.1, there exists $B_{Y} \in \mathcal{B}(M \mid Y)$ such that $X \subseteq B_{Y}$. Suppose that $B_{Y} \notin \mathcal{B}$. Then there exists $f \in E-Y$ such that $B_{Y} \sqcup\{f\} \in \mathcal{J}$.

As $B_{2} \in \mathcal{B}$ does not contain $f$, there exists $C_{1} \in \mathcal{C}(M)$ such that $f \in$ $\mathrm{C}_{1} \subseteq \mathrm{B}_{Y} \sqcup \mathrm{B}_{2} \sqcup\{f\}$. Without loss of generality, $\left|\mathrm{C}_{1}-\left(\mathrm{B}_{Y} \sqcup\{f\}\right)\right|$ is minimum. Suppose that $\left|C_{1}-\left(B_{Y} \sqcup\{f\}\right)\right|$ is positive. Then there exists $e \in\left(C_{1} \cap B_{2}\right)-$ ( $\left.B_{Y} \sqcup\{f\}\right)$, necessarily in $Y$. As $B_{Y} \in \mathcal{B}(M \mid Y)$, there exists $C_{2} \in \mathcal{C}(M)$ such that $e \in \mathrm{C}_{2} \subseteq \mathrm{B}_{Y} \sqcup\{e\}$. So by Strong Circuit Elimination, there exists $\mathrm{C}_{3} \in \mathcal{C}(M)$ such that $f \in \mathrm{C}_{3} \subseteq\left(\mathrm{C}_{1} \cup \mathrm{C}_{2}\right)-\{e\} \subseteq \mathrm{B}_{Y} \sqcup \mathrm{B}_{2} \sqcup\{f\}$. But then

$$
\begin{aligned}
\mathrm{C}_{3}-\left(\mathrm{B}_{Y} \sqcup\{f\}\right) & \subseteq\left(\mathrm{C}_{1} \cup \mathrm{C}_{2}\right)-\left(\mathrm{B}_{Y} \sqcup\{e, f\}\right) \\
& \subseteq \mathrm{C}_{1}-\left(\mathrm{B}_{Y} \sqcup\{e, f\}\right) \\
& \subsetneq \mathrm{C}_{1}-\left(\mathrm{B}_{Y} \sqcup\{f\}\right) .
\end{aligned}
$$

So $\left|C_{3}-\left(B_{Y} \sqcup\{f\}\right)\right|<\left|C_{1}-\left(B_{Y} \sqcup\{f\}\right)\right|$, contradicting the minimality of $\mid C_{1}-$ $\left(B_{Y} \sqcup\{f\}\right) \mid$. Thus $\left|C_{1}-\left(B_{Y} \sqcup\{f\}\right)\right|=0$, and so $C_{1} \subseteq B_{Y} \sqcup\{f\}$, contradicting $\mathrm{B}_{Y} \sqcup\{f\} \in \mathcal{J}$. Hence $\mathrm{B}_{Y} \in \mathcal{B}$, and so Middle Basis holds.

It can be shown that (B1)-(B4) provide an alternative axiomatisation of independence spaces.

Proposition 3.3.5. (B1)-(B3) are equivalent to (B1), (B2) and:

$\left(B^{\prime}\right)$ (Basis Exchange) For every $B_{1}, B_{2} \in \mathcal{B}$ and every $e \in B_{1}$, there exists $\mathrm{f} \in \mathrm{B}_{2}$ such that $\left(\mathrm{B}_{1}-\{\mathrm{e}\}\right) \sqcup\{\mathrm{f}\} \in \mathcal{B}$.

We present the argument given on pp. 30-31 of [NW86]. 
Proof. In order to prove the forward implication, it is helpful to first establish the following claim.

(1) If $\mathrm{B}_{1}, \mathrm{~B}_{2} \in \mathcal{B}$ are such that $\left|\mathrm{B}_{1}-\mathrm{B}_{2}\right|=1$, then $\left|\mathrm{B}_{2}-\mathrm{B}_{1}\right|=1$.

Let $B_{1}, B_{2} \in \mathcal{B}$ be such that $B_{1}-B_{2}=\{e\}$ and let $X=B_{1} \cap B_{2}=$ $B_{1}-\{e\}$. By (B2), there exists $f \in B_{2}-X$. Now,

$$
\mathrm{B}_{2} \supseteq \mathrm{X} \sqcup\{f\} \subseteq \mathrm{B}_{1} \sqcup\{f\} \supseteq \mathrm{B}_{1} .
$$

So by Middle Basis there exists $B_{3} \in \mathcal{B}$ such that

$$
\left(\mathrm{B}_{1}-\{\mathrm{e}\}\right) \sqcup\{\mathrm{f}\}=\mathrm{X} \sqcup\{\mathrm{f}\} \subseteq \mathrm{B}_{3} \subseteq \mathrm{B}_{1} \sqcup\{\mathrm{f}\} .
$$

As $B_{3} \neq B_{1} \sqcup\{f\}$ by (B2), $B_{3}=\left(B_{1}-\{e\}\right) \sqcup\{f\} \subseteq B_{2}$. It follows from (B2) that $B_{3} \not \subset B_{2}$, so $B_{3}=B_{2}$. Thus $\left|B_{2}-B_{1}\right|=\left|\left(\left(B_{1}-\{e\}\right) \sqcup\{f\}\right)-B_{1}\right|=$ 1.

Now let $B_{1}, B_{2} \in \mathcal{B}$ be distinct and let $e \in B_{1}-B_{2}$. Set $X=B_{1}-\{e\}$ and $\mathrm{Y}=\mathrm{B}_{2} \cup \mathrm{X}$. By Middle Basis, there exists $\mathrm{B}_{3} \in \mathcal{B}$ such that $\mathrm{X} \subseteq \mathrm{B}_{3} \subseteq \mathrm{Y}$. Clearly $X=X \cap B_{3}=B_{1} \cap B_{3}$. Consequently $\left|B_{1}-B_{3}\right|=\left|B_{1}-X\right|=1$, so $\left|B_{3}-B_{1}\right|=1$ by (1). Moreover, $B_{3}-B_{1}=B_{3}-X$, so $B_{3}=X \sqcup\{f\}$ for some $f \in Y$, necessarily in $B_{2}$. Hence Basis Exchange holds.

Turning to the reverse implication, let $X \subseteq Y \subseteq E$ and let $B_{1}, B_{2} \in \mathcal{B}$ be such that $X \subseteq B_{1} \neq B_{2} \subseteq Y$. Choose $B_{3} \in \mathcal{B}$ such that $X \subseteq B_{3}$ and $B_{2} \cap B_{3}$ is maximal. Suppose that there exists $e \in B_{3}-Y \subseteq B_{3}-B_{2}$. Then by Basis Exchange, there exists $f \in B_{2}$ such that $\left(B_{3}-\{e\}\right) \sqcup\{f\} \in \mathcal{B}$. Now, $X \subseteq\left(B_{3}-\{e\}\right) \sqcup\{f\}$ and $B_{2} \cap B_{3} \subsetneq B_{2} \cap\left(\left(B_{3}-\{e\}\right) \sqcup\{f\}\right)$, for $e \notin B_{2} \ni f$. This contradicts the choice of $B_{3}$, so $B_{3}-Y=\varnothing$. Hence $B_{3} \subseteq Y$, and so Middle Basis holds.

Say that $Y \subseteq E$ is an J-subset of $X \subseteq E$ if $Y \subseteq X$ and $Y \in \mathcal{J}$. The next result can be shown to hold for independence spaces in general using Rado's Selection Lemma—see pp. 77-78 of [Ox192] for a proof. 
Proposition 3.3.6. Let $\mathrm{M}=(\mathrm{E}, \mathrm{J})$ be an independence space and let $\mathrm{X} \subseteq \mathrm{E}$ have no infinite J-subset. Then the bases of $\mathrm{M} \mid \mathrm{X}$ are equicardinal.

We reproduce the argument given on p. 17 of [Ox104].

Proof. Suppose that the assertion fails, with $B_{X}, B_{X}^{\prime} \in \mathcal{B}(M \mid X)$ such that $\left|B_{X}\right|>\left|B_{X}^{\prime}\right|$ and $\left|B_{X}-B_{X}^{\prime}\right|$ is minimum. Let $e \in B_{X}-B_{X}^{\prime}$. Then by Basis Exchange, there exists $f \in B_{X}^{\prime}$ such that $\left(B_{X}-\{e\}\right) \sqcup\{f\} \in \mathcal{B}(M \mid X)$. Moreover, $\left|\left(B_{X}-\{e\}\right) \sqcup\{f\}\right|=\left|B_{X}\right|>\left|B_{X}^{\prime}\right|$ and $\left|\left(\left(B_{X}-\{e\}\right) \sqcup\{f\}\right)-B_{X}^{\prime}\right|<$ $\left|B_{X}-B_{X}^{\prime}\right|$, contradicting the choice of $\left(B_{X}, B_{X}^{\prime}\right)$. Hence the bases of $M \mid X$ are equicardinal.

Proposition 3.3.7. Let $\mathrm{M}=(\mathrm{E}, \mathrm{J})$ be an independence space, let $\mathrm{Y} \subseteq \mathrm{X} \subseteq \mathrm{E}$ and let $\mathrm{B}_{1}, \mathrm{~B}_{2} \in \mathcal{B}(M \mid(E-X))$. Then $\mathrm{B}_{1} \sqcup \mathrm{Y} \in \mathcal{J}$ if and only if $\mathrm{B}_{2} \sqcup \mathrm{Y} \in \mathcal{J}$.

Proof. It suffices by symmetry to demonstrate one direction only. So suppose that $B_{1} \sqcup \mathrm{Y} \in \mathcal{J}$ and that $B_{2} \sqcup \mathrm{Y} \notin \mathcal{J}$. Let $M^{\prime}=M \mid((E-X) \sqcup Y)$. Clearly $\mathrm{B}_{1} \sqcup \mathrm{Y} \in \mathcal{B}\left(M^{\prime}\right)$. There exists $\mathrm{B} \in \mathcal{B}\left(M^{\prime}\right)$ such that $\mathrm{B}_{2} \subseteq \mathrm{B} \subseteq \mathrm{B}_{2} \sqcup \mathrm{Y}$ by Proposition 3.3.1. Necessarily $B=B_{2} \sqcup Y^{\prime}$ for some $Y^{\prime} \subsetneq Y$. Let $y \in Y-Y^{\prime}$. Then $y \in\left(B_{1} \sqcup Y\right)-B$, so by Basis Exchange there exists $\mathrm{b} \in \mathrm{B}-\left(\mathrm{B}_{1} \sqcup \mathrm{Y}\right)$ such that $\left(\left(\mathrm{B}_{1} \sqcup \mathrm{Y}\right)-\{\mathrm{y}\}\right) \sqcup\{\mathrm{b}\} \in \mathcal{B}\left(\mathrm{M}^{\prime}\right)$. As $\mathrm{Y}^{\prime} \subseteq \mathrm{Y}$, $\mathrm{b} \in \mathrm{B}_{2}-\mathrm{B}_{1}$, so $\mathrm{B}_{1} \subsetneq \mathrm{B}_{1} \sqcup\{\mathrm{b}\} \subseteq \mathrm{E}-\mathrm{X}$ and $\mathrm{B}_{1} \sqcup\{\mathrm{b}\} \in \mathcal{J}(M \mid(E-X))$, contradicting $\mathrm{B}_{1} \in \mathcal{B}(M \mid(E-X))$. Hence $\mathrm{B}_{2} \sqcup \mathrm{Y} \in \mathcal{J}$.

\subsection{Finitary Rank}

Given equicardinality of the bases of $M \mid X$ for every $X \subseteq E(M)$ (a generalisation of Proposition 3.3.6), it is possible to define a (full) rank function for the independence space $M$ by setting the rank of $X$ to be the (common) cardinality of its maximal $\mathcal{J}(M)$-subsets.

However, as the structure of any given independence space is determined locally (by Finite Character), independence spaces can in fact be 
axiomatised by finitary rank. This section is devoted to such an axiomatisation. The (finitary) rank functions developed yield the necessary properties of rank without requiring a Rado's Selection Lemma argument like that given on pp. 77-78 of [Ox192].

Recall that $\mathcal{P}(E)$ denotes the power set of a set $E$. The following definition is sensible as a consequence of Proposition 3.3.6.

Definition 3.4.1. Let $M=(E, \mathcal{J})$ be an independence space. Then the (finitary) rank function of $M$ is the function $r_{M}: \mathcal{P}(E) \rightarrow \omega \sqcup\{\infty\}$ defined by

$$
r_{M}(X)= \begin{cases}\left|B_{X}\right| \text { for any } B_{X} \in \mathcal{B}(M \mid X) & \text { if } X \text { has no infinite J-subset, and } \\ \infty & \text { otherwise }\end{cases}
$$

for every $X \subseteq E$. The subscript " $M$ " is omitted when there is no ambiguity. The (finitary) rank of $X \subseteq E$ is $r_{M}(X)$, and the (finitary) rank of $M$, denoted $r(M)$, is the rank of $E$.

For every set $\mathrm{E}$, let

$$
\#(E)= \begin{cases}|E| & \text { if } E \text { is finite, and } \\ \infty & \text { otherwise. }\end{cases}
$$

Recall that for every set $E$, a function $\psi: \mathcal{P}(E) \rightarrow \mathbb{R} \sqcup\{\infty\}$ is submodular if $\psi(X \cap Y)+\psi(X \cup Y) \leqslant \psi(X)+\psi(Y)$ for every $X, Y \subseteq E$.

Proposition 3.4.2. Let $M$ be an independence space. Then:

(R1) $r(X) \leqslant \#(X)$ for every $X \subseteq E$.

(R2) $r(X) \leqslant r(Y)$ whenever $X \subseteq Y \subseteq E$.

(R3) $r$ is submodular.

(R4) $r(X)=\sup \{r(Y) \mid Y \subseteq X$ is finite $\}$ for every $X \subseteq E$. 
Proof. The statements (R1) and (R2) follow immediately from the definition of the rank function, so we demonstrate (R3) and (R4).

Let $X, Y \subseteq E$. Clearly $r(X \cap Y)+r(X \cup Y)=\infty$ forces $r(X)+r(Y)=\infty$, so suppose that all four ranks are finite. Let $B_{X \cap Y} \in \mathcal{B}(M \mid X \cap Y)$. By Proposition 3.3.1, this is contained in some $B_{X \cup Y} \in \mathcal{B}(M \mid X \cup Y)$. It follows from (I2) that $\mathrm{B}_{X \cup Y} \cap \mathrm{X} \subseteq \mathrm{X}$ and $\mathrm{B}_{X \cup Y} \cap \mathrm{Y} \subseteq \mathrm{Y}$ are each independent, so $\left|B_{X \cup Y} \cap X\right| \leqslant r(X)$ and $\left|B_{X \cup Y} \cap Y\right| \leqslant r(Y)$. Consequently

$$
\begin{aligned}
r(X)+r(Y) \geqslant & \left|B_{X \cup Y} \cap X\right|+\left|B_{X \cup Y} \cap Y\right| \\
= & \left|\left(B_{X \cup Y} \cap X\right) \cup\left(B_{X \cup Y} \cap Y\right)\right| \\
& +\left|\left(B_{X \cup Y} \cap X\right) \cap\left(B_{X \cup Y} \cap Y\right)\right| \\
= & \left|B_{X \cup Y}\right|+\left|B_{X \cap Y}\right| \\
= & r(X \cup Y)+r(X \cap Y) .
\end{aligned}
$$

Hence (R3) holds.

Now let $X \subseteq E$. If $r(X)=\infty$, then $X$ has an infinite J-subset I. Every finite $Y \subseteq I$ has $Y \in \mathcal{B}(M \mid Y)$, so $r(Y)=|Y|$. Thus $\sup \{r(Y) \mid Y \subseteq X$ is finite $\}=$ $\infty$ also. Otherwise, if $r(X) \in \omega$, then $r(X)=\left|B_{X}\right|$ for some $B_{X} \in \mathcal{B}(M \mid X)$. As $B_{X}$ is finite, $r(X) \leqslant \sup \{r(Y) \mid Y \subseteq X$ is finite $\}$. It follows from (R2) that $r(X) \geqslant \sup \{r(Y) \mid Y \subseteq X$ is finite $\}$, so equality holds. Consequently (R4) holds.

The finite independent sets of an independence space are easily characterised by rank.

Lemma 3.4.3. Let $\mathrm{M}=(\mathrm{E}, \mathrm{J})$ be an independence space and let $\mathrm{X} \subseteq \mathrm{E}$ be finite. Then $\mathrm{X} \in \mathcal{J}$ if and only if $\mathrm{r}(\mathrm{X})=|\mathrm{X}|$.

Proof. Suppose that $X \in \mathcal{J}$. Then $X \in \mathcal{B}(M \mid X)$, so $r(X)=|X|$.

Now suppose that $r(X)=|X|$. Let $B_{X} \in \mathcal{B}(M \mid X)$. Then $\left|B_{X}\right|=r(X)=$ $|X|$, so $X=B_{X} \in \mathcal{J}$.

However, this correspondence does not extend to infinite sets. 
Example 3.4.4. Let $E$ be an infinite set, let $C \subseteq E$ have size 2 and let $\mathcal{J}=\{X \subseteq E \mid C \nsubseteq X\}$. Then it is easily seen that $(E, \mathcal{J})$ is an independence space for which $E$ is dependent. However, as $E-C \in \mathcal{J}$ is infinite, $r(E)=$ $\infty=\#(E)$.

Lemma 3.4.5. Let $E$ be a set, let $r: \mathcal{P}(E) \rightarrow \mathbb{R} \sqcup\{\infty\}$ satisfy (R2) and (R3) and let $\mathrm{X}, \mathrm{Y} \subseteq \mathrm{E}$ be such that $|\mathrm{Y}-\mathrm{X}| \in \omega$ and $\mathrm{r}(\mathrm{X} \sqcup\{\mathrm{y}\})=\mathrm{r}(\mathrm{X}) \in \omega$ for every $y \in Y-X$. Then $r(X \cup Y)=r(X)$.

We reproduce the proof given on p. 24 of [Ox104].

Proof. Write $\mathrm{Y}-\mathrm{X}=\left\{\mathrm{y}_{1}, \ldots, \mathrm{y}_{\mathrm{m}}\right\}$. We procede by induction on $\mathrm{m}$. Clearly the result holds if $m \leqslant 1$, so suppose that it is true for all $m \leqslant n$ and let $m=n+1$. Then by the induction assumption,

$$
\begin{array}{rlrl}
2 r(X)= & r\left(X \sqcup\left\{y_{1}, \ldots, y_{n}\right\}\right)+r\left(X \sqcup\left\{y_{n+1}\right\}\right) & \\
\geqslant & r\left(\left(X \sqcup\left\{y_{1}, \ldots, y_{n}\right\}\right) \cup\left(X \sqcup\left\{y_{n+1}\right\}\right)\right) & \\
& +r\left(\left(X \sqcup\left\{y_{1}, \ldots, y_{n}\right\}\right) \cap\left(X \sqcup\left\{y_{n+1}\right\}\right)\right) & & \text { by (R3) } \\
= & r(X \cup Y)+r(X) & \\
\geqslant & 2 r(X) & & \text { by (R2). }
\end{array}
$$

Consequently $r(X \cup Y)=r(X)$.

Denote the collection of all finite subsets of a set $E$ by $\mathcal{P}_{\text {fin }}(E)$.

Proposition 3.4.6. Let $\mathrm{E}$ be a set, let $\mathrm{r}: \mathcal{P}_{\mathrm{fin}}(\mathrm{E}) \rightarrow \omega$ satisfy $(\mathrm{R} 1)-(\mathrm{R} 3)$ and let

$$
\mathcal{J}=\{\mathrm{I} \subseteq \mathrm{E}: \mathrm{r}(\mathrm{X})=|\mathrm{X}| \text { for every finite } \mathrm{X} \subseteq \mathrm{I}\}
$$

Then $(\mathrm{E}, \mathrm{J})$ is an independence space.

Proof. Statement (I1) follows from (R1), while (I2) and (I4) are consequences of the definition of $\mathcal{J}$. So we demonstrate (I3): let $\mathrm{I}_{1}, \mathrm{I}_{2} \in \mathcal{J}$ be 
such that $\left|\mathrm{I}_{1}\right|<\left|\mathrm{I}_{2}\right| \in \omega$ and suppose that $\mathrm{I}_{1} \sqcup\{e\} \notin \mathcal{J}$ for each $e \in \mathrm{I}_{2}-\mathrm{I}_{1}$. Then $r\left(\mathrm{I}_{1} \sqcup\{e\}\right)<\left|\mathrm{I}_{1} \sqcup\{e\}\right|$ for each $e \in \mathrm{I}_{2}-\mathrm{I}_{1}$ by Lemma 3.4.3, so

$$
\begin{aligned}
\left|\mathrm{I}_{1}\right|=\mathrm{r}\left(\mathrm{I}_{1}\right) \leqslant & r\left(\mathrm{I}_{1} \sqcup\{e\}\right) \leqslant\left|\mathrm{I}_{1}\right| \\
& \mathrm{r}\left(\mathrm{I}_{1} \sqcup\{e\}\right)=\left|\mathrm{I}_{1}\right| .
\end{aligned}
$$

It follows from Lemma 3.4.5 that $r\left(\mathrm{I}_{1} \cup \mathrm{I}_{2}\right)=\left|\mathrm{I}_{1}\right|$. But then

$$
\left|\mathrm{I}_{2}\right|=r\left(\mathrm{I}_{2}\right) \leqslant r\left(\mathrm{I}_{1} \cup \mathrm{I}_{2}\right)=\left|\mathrm{I}_{1}\right|<\left|\mathrm{I}_{2}\right|
$$

—a contradiction. Hence (I3) holds.

While every function $r$ satisifying (R1)-(R3) on $\mathcal{P}_{\text {fin }}(E)$ for some set $E$ yields an independence space, this may not have rank function $r$. In particular, $r(X)$ may be infinite when $X \subseteq E$ has finite rank, making $r$ poor for distinguishing between finite- and infinite-rank subsets of $E$.

Example 3.4.7. Let $E$ be an infinite set. Define $r: \mathcal{P}(E) \rightarrow \omega \sqcup\{\infty\}$ by

$$
r(X)= \begin{cases}0 & \text { if } X \text { is finite, and } \\ \infty & \text { otherwise }\end{cases}
$$

for every $X \subseteq E$. It is easily seen that $r$ satisfies (R1)-(R3). Let

$$
\begin{aligned}
\mathcal{J} & =\{I \subseteq E: r(X)=|X| \text { for every finite } X \subseteq I\} \\
& =\{\varnothing\} .
\end{aligned}
$$

Then $M:=(E, \mathcal{J})$ is an independence space by Proposition 3.4.6. However, $r_{M}(E)=0 \neq \infty=r(E)$.

However, additionally assuming (R4) forces equality.

Proposition 3.4.8. Let $\mathrm{E}$ be a set, let $\mathrm{r}: \mathcal{P}(\mathrm{E}) \rightarrow \omega \sqcup\{\infty\}$ satisfy $(R 1)-(R 4)$ and let

$$
\mathcal{J}=\{\mathrm{I} \subseteq \mathrm{E}: \mathrm{r}(\mathrm{X})=|\mathrm{X}| \text { for every finite } \mathrm{X} \subseteq \mathrm{I}\}
$$


Then $M:=(E, \mathcal{J})$ is an independence space with $r_{M}=r$.

Proof. Proposition 3.4.6 shows that $M$ is an independence space. We first demonstrate the following claim.

(1) $r=r_{M}$ on $\mathcal{P}_{f i n}(E)$.

Let $X \subseteq E$ be finite and suppose that $X \in \mathcal{J}$. Then $r(X)=|X|=r_{M}(X)$ by the definition of $\mathcal{J}$ and Lemma 3.4.3.

Now suppose that $X \notin \mathcal{J}$. Let $B_{X} \in \mathcal{B}(M \mid X)$. Then $B_{X} \in \mathcal{J}$ is finite, so $r(X)=\left|B_{X}\right|=r_{M}(X)$ by the definitions of $\mathcal{J}$ and $r_{M}$.

Hence $r=r_{M}$ on $\mathcal{P}_{\text {fin }}(E)$.

Now let $X \subseteq E$ be arbitrary. Then

$$
\begin{aligned}
r(X) & =\sup \{r(Y) \mid Y \subseteq X \text { is finite }\} & & \text { by (R4) } \\
& =\sup \left\{r_{M}(Y) \mid Y \subseteq X \text { is finite }\right\} & & \text { by (1) } \\
& =r_{M}(X) & & \text { by (R4). }
\end{aligned}
$$

It follows from Propositions 3.4.2 and 3.4.8 that independence spaces are characterised by their rank functions; that is, there exists a one-to-one correspondence

\{Independence spaces on $\mathrm{E}\}$

$\longleftrightarrow\{$ Functions $r: \mathcal{P}(E) \rightarrow \omega \sqcup\{\infty\}$ satisfying $(\mathrm{R} 1)-(\mathrm{R} 4)\}$.

Every independence space is completely determined by its finite independent sets (due to Finite Character). It follows from Lemma 3.4.3 that every independence space $M$ on $E$ is completely determined by $\left.r_{M}\right|_{\mathcal{P}_{f i n}(E)}$.

On the other hand, every function $r: \mathcal{P}_{f i n}(E) \rightarrow \omega$ satisfying (R1)(R3) determines an independence space $M$ on $E$. Moreover, $M$ has $r_{M}=$ $r$ on $\mathcal{P}_{\text {fin }}(E)$ by Claim (1) in the proof of Proposition 3.4.8. It follows 
that $r$ completely determines $M$. Consequently there exists a one-to-one correspondence

\{Independence spaces on $\mathrm{E}\}$

$\longleftrightarrow\left\{\right.$ Functions $r: \mathcal{P}_{\text {fin }}(\mathrm{E}) \rightarrow \omega$ satisfying $\left.(\mathrm{R} 1)-(\mathrm{R} 3)\right\}$,

and so (R4) is unnecessary for characterising independence spaces by rank. However, including (R4) as a rank axiom results in a characterisation under which infinite sets may easily be classified as having finite rank or infinite rank. This distinction is crucial for establishing results such as Proposition 5.2.7.

Lemma 3.4.9. Let $M$ be a finite-rank independence space on $E=E_{1} \sqcup E_{2} \sqcup E_{3}$, let $\mathrm{k} \in \mathrm{\omega}$ and suppose that

$$
r\left(E_{i}\right)+r\left(E-E_{i}\right) \leqslant r(M)+k
$$

for each $i=1,2,3$. Then

$$
\sum_{i=1}^{3} r\left(E-E_{i}\right)-2 r(M) \leqslant 2 k .
$$

Proof. We simply apply the hypothesis and (R3).

$$
\begin{aligned}
\sum_{i=1}^{3} r\left(E-E_{i}\right)-2 r(M)= & r\left(E_{2} \sqcup E_{3}\right)+\left(r\left(E_{1} \sqcup E_{3}\right)-r(M)\right) \\
& +\left(r\left(E_{1} \sqcup E_{2}\right)-r(M)\right) \\
\leqslant & r\left(E_{2} \sqcup E_{3}\right)+\left(k-r\left(E_{2}\right)\right) \\
& +\left(k-r\left(E_{3}\right)\right) \\
\leqslant & 2 k .
\end{aligned}
$$

A short discussion of (finite) rank functions $\mathcal{P}_{\text {fin }}(E) \rightarrow \omega$ is given 
on pp. 100-102 of [FF00]. A nice treatment of rank functions of preindependence spaces is given in [PP70].

\subsection{Closure and Flats}

Definition 3.5.1. Let $M=(E, \mathcal{J})$ be an independence space. Then the closure operator of $M$ is the operator $\mathrm{cl}_{M}: \mathcal{P}(E) \rightarrow \mathcal{P}(E)$ defined by

$$
\operatorname{cl}_{M}(X)=X \cup\{e \in E \mid(\exists I \in \mathcal{I}) \mathrm{I} \subseteq X \text { and } \mathrm{I} \sqcup\{e\} \notin \mathcal{J}\}
$$

for every $X \subseteq E$. The subscript " $M$ " is omitted when there is no ambiguity.

A flat or closed set of $\mathrm{M}$ is a subset $\mathrm{F} \subseteq \mathrm{E}$ for which $\mathrm{cl}(\mathrm{F})=\mathrm{F}$. A flat $\mathrm{F}$ is proper if $\mathrm{F} \neq \mathrm{E}$. A hyperplane of $\mathrm{M}$ is a maximal proper flat of $\mathrm{M}$. The collections of flats and hyperplanes of $M$ are denoted by $\mathcal{F}(M)$ and $\mathcal{H}(M)$ respectively. A subset $X \subseteq E$ spans $Y \subseteq E$ if $Y \subseteq \mathrm{cl}(X)$, and $X$ is spanning if it spans $E$.

Proposition 3.5.2. Let $\mathrm{M}=(\mathrm{E}, \mathrm{J})$ be an independence space. Then:

(CL1) $\mathrm{X} \subseteq \mathrm{cl}(\mathrm{X})$ for every $\mathrm{X} \subseteq \mathrm{E}$.

(CL2) $\operatorname{cl}(\mathrm{X}) \subseteq \mathrm{cl}(\mathrm{Y})$ whenever $\mathrm{X} \subseteq \mathrm{Y} \subseteq \mathrm{E}$.

(CL3) $\operatorname{cl}(\operatorname{cl}(\mathrm{X}))=\operatorname{cl}(\mathrm{X})$ for every $\mathrm{X} \subseteq \mathrm{E}$.

(CL4) Whenever $\mathrm{X}, \mathrm{Y} \subseteq \mathrm{E}$ and $\mathrm{e} \in \mathrm{cl}(\mathrm{Y})-\mathrm{cl}(\mathrm{Y}-\mathrm{X})$, there exists $\mathrm{x} \in \mathrm{X}$ such that $x \in \operatorname{cl}((Y-\{x\}) \cup\{e\})$.

(CL5) Whenever $\mathrm{X} \subseteq \mathrm{E}$ and $\mathrm{e} \in \mathrm{cl}(\mathrm{X})$, there exists finite $\mathrm{Y} \subseteq \mathrm{X}$ such that $e \in \operatorname{cl}(Y)$.

Our argument closely follows that given on pp. 83-84 of [Ox192]. 
Proof. The statements (CL1) and (CL2) follow immediately from the definition of the closure operator. In order to demonstrate (CL3), it is helpful to first establish that for every $X \subseteq E$ and every $B_{X} \in \mathcal{B}(M \mid X)$,

$$
\mathrm{cl}(\mathrm{X})= \begin{cases}\mathrm{X} \sqcup\{e \in \mathrm{E} \mid \mathrm{X} \sqcup\{\mathrm{e}\} \notin \mathcal{J}\} & \text { if } \mathrm{X} \in \mathcal{J}, \text { and } \\ \mathrm{cl}\left(\mathrm{B}_{X}\right) & \text { otherwise. }\end{cases}
$$

Clearly (3.5.1) holds when $X \in \mathcal{J}$, so suppose otherwise. It follows from (CL2) that $\mathrm{cl}\left(\mathrm{B}_{X}\right) \subseteq \mathrm{cl}(X)$. If $x \in X-\mathrm{B}_{X}$, then $\mathrm{B}_{X} \sqcup\{x\} \notin \mathcal{J}$, so $x \in \mathrm{cl}\left(\mathrm{B}_{X}\right)$. Thus $X \subseteq \operatorname{cl}\left(B_{X}\right)$. Now suppose that $e \in \operatorname{cl}(X)-X$. Then $I \sqcup\{e\} \notin \mathcal{J}$ for some J-subset I of $X$. If $\mathrm{B}_{X} \sqcup\{\boldsymbol{e}\} \notin \mathcal{J}$, then $e \in \operatorname{cl}\left(\mathrm{B}_{X}\right)$, so suppose that $\mathrm{B}_{X} \sqcup\{e\} \in \mathcal{J}$. Then $\mathrm{B}_{X} \sqcup\{e\} \in \mathcal{B}(M \mid(X \sqcup\{e\}))$.

Moreover, I is contained in some $\mathrm{B} \in \mathcal{B}(\mathrm{M} \mid(\mathrm{X} \sqcup\{\boldsymbol{e}\}))$ not containing e. Thus $e \in\left(B_{X} \sqcup\{e\}\right)-B$, so by Basis Exchange there exists $b \in B-$ $\left(B_{X} \sqcup\{e\}\right)$ such that $\left(\left(B_{X} \sqcup\{e\}\right)-\{e\}\right) \sqcup\{b\}=B_{X} \sqcup\{b\} \in \mathcal{B}(M \mid(X \sqcup\{e\}))$. As $\mathrm{B}_{X} \sqcup\{\mathrm{b}\} \subseteq \mathrm{X}$, this contradicts $\mathrm{B}_{X} \in \mathcal{B}(M \mid X)$. Hence (3.5.1) holds.

It is now easy to demonstrate (CL3): let $X \subseteq E$ and let $B_{X} \in \mathcal{B}(M \mid X)$. Then $\mathrm{cl}(\mathrm{X})=\mathrm{cl}\left(\mathrm{B}_{\mathrm{X}}\right)=\mathrm{B}_{\mathrm{X}} \sqcup\left\{e \in \mathrm{E} \mid \mathrm{B}_{\mathrm{X}} \sqcup\{e\} \notin \mathcal{J}\right\}$ by (3.5.1), so $\mathrm{B}_{\mathrm{X}} \in$ $\mathcal{B}(\mathrm{M} \mid \mathrm{cl}(\mathrm{X}))$. Consequently $\mathrm{cl}(\mathrm{cl}(\mathrm{X}))=\mathrm{cl}\left(\mathrm{B}_{\mathrm{X}}\right)=\mathrm{cl}(\mathrm{X})$, and so (CL3) holds.

Turning to (CL4), let $X, Y \subseteq E$ and let $e \in \operatorname{cl}(Y)-\operatorname{cl}(Y-X)$. Set $\mathrm{X}^{\prime}=\mathrm{X} \cap \mathrm{Y}$. Then $e \in \mathrm{cl}(\mathrm{Y})-\mathrm{cl}\left(\mathrm{Y}-\mathrm{X}^{\prime}\right)$. Clearly (CL4) holds if $e \in \mathrm{X}^{\prime}$, so suppose otherwise. Let $B_{Y-X^{\prime}} \in \mathcal{B}_{Y-X^{\prime}}$. Then $B_{Y-X^{\prime}} \subseteq B_{Y}$ for some $B_{Y} \in$ $\mathcal{B}(M \mid Y)$, so as $e \in \operatorname{cl}(Y)=\operatorname{cl}\left(B_{Y}\right), B_{Y} \sqcup\{e\} \notin \mathcal{J}$. Thus $B_{Y} \in \mathcal{B}\left(M \mid\left(B_{Y} \sqcup\{e\}\right)\right)$.

Now, $e \notin \operatorname{cl}\left(Y-X^{\prime}\right)=\operatorname{cl}\left(B_{Y-X^{\prime}}\right)$, so $B_{Y-X^{\prime}} \sqcup\{e\} \in \mathcal{J}$. Thus $B_{Y-X^{\prime}} \sqcup\{e\} \subseteq$ $\mathrm{B}_{Y}^{\prime}$ for some $\mathrm{B}_{Y}^{\prime} \in \mathcal{B}(M \mid(Y \sqcup\{e\}))$. If $X^{\prime} \subseteq \mathrm{B}_{Y}^{\prime}$, then

$$
\mathrm{B}_{Y}^{\prime} \supseteq \mathrm{B}_{Y-X^{\prime}} \sqcup \mathrm{X}^{\prime} \sqcup\{e\} \supsetneq \mathrm{B}_{Y-X^{\prime}} \sqcup \mathrm{X}^{\prime} \supseteq \mathrm{B}_{Y}
$$

—a contradiction. So there exists $x \in X^{\prime}-B_{Y}^{\prime}$. Necessarily

$$
x \in \operatorname{cl}(Y) \subseteq \operatorname{cl}(Y \cup\{e\})=\operatorname{cl}\left(B_{Y}^{\prime}\right)=\operatorname{cl}((Y-\{x\}) \cup\{e\})
$$


and so (CL4) holds.

Finally, let $X \subseteq E$ and let $e \in \operatorname{cl}(X)$. Clearly (CL5) holds if $e \in X$, so suppose not. Then there exists an J-subset I of $X$ such that $I \sqcup\{e\} \notin \mathcal{J}$. It follows from (I4) that $I \sqcup\{e\}$ contains a (finite) circuit $J \sqcup\{e\}$, whence $e \in \mathrm{cl}(\mathrm{J})$ and $\mathrm{J} \subseteq \mathrm{X}$ is finite. Hence (CL5) holds.

Lemma 3.5.3. Let $M$ be an independence space and let $X, Y \subseteq E(M)$. Then $\operatorname{cl}(X \cup \operatorname{cl}(Y))=\operatorname{cl}(X \cup Y)$.

Proof. Let $B_{Y} \in \mathcal{B}(M \mid Y)$ and let $B_{X \cup Y} \in \mathcal{B}(M \mid(X \cup Y))$ contain $B_{Y}$. Then $\mathrm{B}_{X \cup Y} \in \mathcal{B}(\mathrm{M} \mid(\mathrm{X} \cup \mathrm{cl}(\mathrm{Y})))$, for otherwise there would exist $e \in \mathrm{cl}(\mathrm{Y})-(\mathrm{X} \cup$ $Y)$ such that $B_{X \cup Y} \sqcup\{e\} \in \mathcal{J}(M)$, contradicting $B_{Y} \sqcup\{e\} \notin \mathcal{J}(M)$. It then follows from (3.5.1) that

$$
\operatorname{cl}(X \cup \operatorname{cl}(Y))=\operatorname{cl}(B X \cup Y)=\operatorname{cl}(X \cup Y) .
$$

Lemma 3.5.4. Let $M$ be an independence space and let $X \subseteq E(M)$. Then $\mathrm{r}(\mathrm{X})=\mathrm{r}(\mathrm{cl}(\mathrm{X}))$.

Proof. If $X$ contains an infinite $\mathcal{J}(M)$-subset, then so does $\mathrm{cl}(\mathrm{X})$ by (CL1). Thus $r(X)=\infty=r(c l(X))$. Otherwise, let $B_{X} \in \mathcal{B}(M \mid X)$. Then $B_{X} \in$ $\mathcal{B}(\mathrm{M} \mid \mathrm{cl}(\mathrm{X}))$ by (3.5.1), so $r(X)=\left|\mathrm{BX}_{X}\right|=r(\mathrm{cl}(X))$.

Proposition 3.5.5. Let $M$ be an independence space and let $Y \subseteq X \subseteq E(M)$. Then $\mathrm{cl}_{M \mid X}(\mathrm{Y})=\mathrm{X} \cap \mathrm{cl}_{M}(\mathrm{Y})$.

Proof.

$$
\begin{aligned}
\mathrm{cl}_{M \mid X}(Y) & =Y \cup\{x \in X \mid(\exists I \in \mathcal{J}(M \mid X)) I \subseteq Y \text { and } \mathrm{I} \sqcup\{x\} \notin \mathcal{J}(M \mid X)\} \\
& =Y \cup\{x \in X \mid(\exists I \in \mathcal{J}(M)) I \subseteq Y \text { and } \mathrm{I} \sqcup\{x\} \notin \mathcal{J}(M)\} \\
& =X \cap c_{M}(Y) .
\end{aligned}
$$

Corollary 3.5.6. Let $\mathrm{M}$ be an independence space and let $\mathrm{X}, \mathrm{F} \subseteq \mathrm{E}(\mathrm{M})$. Then $\mathrm{F} \in \mathcal{F}(\mathrm{M} \mid \mathrm{X})$ if and only if $\mathrm{X} \cap \mathrm{cl}_{\mathrm{M}}(\mathrm{F}) \subseteq \mathrm{F} \subseteq \mathrm{X}$. 
Proof. As $F \in \mathcal{F}(M \mid X)$ if and only if $\mathrm{cl}_{M \mid X}(F) \subseteq F \subseteq X$, it follows from Proposition 3.5.5 that $F \in \mathcal{F}(M \mid X)$ if and only if $X \cap \mathrm{cl}_{M}(F) \subseteq F \subseteq X$.

The next lemma follows immediately from (CL1) and (CL2).

Lemma 3.5.7. Let $M$ be an independence space and let $F, F^{\prime} \in \mathcal{F}(M)$. Then $\mathrm{F} \cap \mathrm{F}^{\prime} \in \mathcal{F}(\mathrm{M})$.

Lemma 3.5.8. Let $M$ be an independence space on $E$, let $X \subseteq E$ and let $e \in$ $\mathrm{E}-\mathrm{cl}(\mathrm{X})$. Then $\mathrm{cl}(\mathrm{X}) \in \mathcal{H}(\mathrm{M} \mid \mathrm{cl}(\mathrm{X} \sqcup\{\mathrm{e}\}))$.

Proof. It follows from Corollary 3.5.6 that $\operatorname{cl}(X) \in \mathcal{F}(M \mid \operatorname{cl}(X \sqcup\{\boldsymbol{e}\}))$. Let $f \in \operatorname{cl}(X \sqcup\{e\})-\operatorname{cl}(X)$. Then

$$
\begin{aligned}
\mathrm{X} \sqcup\{f\} & \subseteq \operatorname{cl}(X \sqcup\{\boldsymbol{e}\}) & & \text { by (CL1) } \\
\mathrm{cl}(\mathrm{X} \sqcup\{f\}) & \subseteq \operatorname{cl}(\mathrm{cl}(\mathrm{X} \sqcup\{\mathbf{e}\})) & & \text { by (CL2) } \\
& =\operatorname{cl}(\mathrm{X} \sqcup\{\mathbf{e}\}) & & \text { by (CL3). }
\end{aligned}
$$

On the other hand, $e \in \operatorname{cl}(X \sqcup\{f\})$ by (CL4), so $\operatorname{cl}(X \sqcup\{e\}) \subseteq \operatorname{cl}(X \sqcup\{f\})$ by the argument above. It follows that

$$
\begin{aligned}
\operatorname{cl}(X \sqcup\{e\}) & =\operatorname{cl}(X \sqcup\{f\}) \\
& =\operatorname{cl}(\operatorname{cl}(X) \sqcup\{f\}) \quad \text { by Lem. 3.5.3. }
\end{aligned}
$$

Hence $\operatorname{cl}(\mathrm{X}) \in \mathcal{H}(\mathrm{M} \mid \mathrm{cl}(\mathrm{X} \sqcup\{\mathrm{e}\}))$.

\subsection{Contraction}

Definition 3.6.1. Let $M=(E, \mathcal{J})$ be an independence space, let $X \subseteq E$ and let $B_{X} \in \mathcal{B}(M \mid X)$. Then the contraction of $M$ by $X$, denoted $M / X$, is $\left(E-X,\left\{Y \subseteq E-X \mid Y \sqcup B_{X} \in \mathcal{J}\right\}\right)$.

Note that this definition is independent of the choice of $B_{X}$ by Proposition 3.3.7. 
Proposition 3.6.2. Every contraction of an independence space is an independence space.

We reproduce the argument given on pp. 76-77 of [Ox192].

Proof. Let $\mathrm{M}=(\mathrm{E}, \mathrm{J})$ be an independence space, let $\mathrm{X} \subseteq \mathrm{E}$ and let $\mathrm{BX} \in$ $\mathcal{B}(M \mid X)$ be fixed. As the statements (I1) and (I2) clearly hold, we consider (I3) and (I4).

Let $\mathrm{I}_{1}, \mathrm{I}_{2} \in \mathcal{J}(\mathrm{M} / \mathrm{X})$ be such that $\left|\mathrm{I}_{1}\right|<\left|\mathrm{I}_{2}\right| \in \omega$. Then $\mathrm{I}_{1} \sqcup \mathrm{B}_{\mathrm{X}}, \mathrm{I}_{2} \sqcup \mathrm{B}_{X} \in$ J. For every finite $B_{0} \subseteq B_{X}$, it follows from (I3) for $M \mid\left(I_{1} \cup I_{2} \cup B_{0}\right)$ that there exists $e \in I_{2}-I_{1}$ such that $I_{1} \sqcup\{e\} \sqcup B_{0} \in \mathcal{J}$, so

$$
\mathrm{X}_{\mathrm{B}_{0}}:=\left\{e \in \mathrm{I}_{2}-\mathrm{I}_{1} \mid \mathrm{I}_{1} \sqcup\{e\} \sqcup \mathrm{B}_{0} \in \mathcal{J}\right\}
$$

is both non-empty and finite. Let $J \subseteq \mathcal{P}_{\text {fin }}\left(B_{X}\right)$ be finite, let $B^{\prime}=\bigcup J$ and let $e_{J} \in X_{B^{\prime}}$. Then $e_{J} \in \bigcap_{j \in J} X_{j}$ by (I2). Define a choice function $f_{J}$ for $\left\{X_{j} \mid\right.$ $j \in J\}$ by $f_{J}(j)=e_{J}$ for every $j \in J$. Let $f$ be as per Rado's Selection Lemma.

Let $J=\{i, j\} \subseteq \mathcal{P}_{\text {fin }}\left(B_{X}\right)$. Then $\left.f\right|_{J}=\left.f_{K}\right|_{J}$ for some finite $K \subseteq \mathcal{P}_{\text {fin }}\left(B_{X}\right)$ containing $J$. As $f_{K}(i)=f_{K}(j)$ by definition, it follows that $f(i)=f(j)$. Consequently $\operatorname{im}(f)=\{e\}$ for some $e \in I_{2}-I_{1}$. But then $I_{1} \sqcup\{e\} \sqcup B_{0} \in \mathcal{J}$ for every finite $B_{0} \subseteq B_{X}$, so $Z \in \mathcal{J}$ for every finite $Z \subseteq I_{1} \sqcup\{e\} \sqcup B_{X}$. It follows from (I4) that $\mathrm{I}_{1} \sqcup\{\mathfrak{e}\} \sqcup \mathrm{B}_{X} \in \mathcal{J}$, so $\mathrm{I}_{1} \sqcup\{e\} \in \mathcal{J}(M / X)$. Hence (I3) holds.

Turning to (I4), let $Y \subseteq E-X$ such that $Z \in \mathcal{J}(M / X)$ for every finite $Z \subseteq Y$. Then $Z \sqcup B_{X} \in \mathcal{J}$ for every finite $Z \subseteq Y$, so $Z \in \mathcal{J}$ for every finite $Z \subseteq Y \sqcup B_{X}$ by (I2). Thus $Y \sqcup B_{X} \in \mathcal{J}$ by (I4), so $Y \in \mathcal{J}(M / X)$. Consequently (I4) holds.

Proposition 3.6.3. Let $\mathrm{M}$ be an independence space and let $\mathrm{X}, \mathrm{Y} \subseteq \mathrm{E}(\mathrm{M})$ be disjoint. Then

(1) $(M \backslash X) \backslash Y=M \backslash(X \sqcup Y)=(M \backslash Y) \backslash X$,

(2) $(M / X) / Y=M /(X \sqcup Y)=(M / Y) / X$, and 
(3) $(M \backslash X) / Y=(M / Y) \backslash X$.

Proof. Write $E=E(M)$. As (1) is trivial, we consider (2) and (3). In order to establish (2), it suffices by symmetry to demonstrate that $(M / X) / Y=$ $M /(X \sqcup Y)$. Clearly each of these independence spaces has ground set $E-(X \sqcup Y)$. Let $B_{X} \in \mathcal{B}(M \mid X)$ and let $B \in \mathcal{B}((M / X) \mid Y)$. Then

$$
\mathcal{J}((M / X) / Y)=\left\{Z \subseteq E-(X \sqcup Y) \mid Z \sqcup B_{X} \sqcup B \in \mathcal{J}(M)\right\}
$$

Clearly $B_{X} \sqcup B \in \mathcal{J}(M \mid(X \sqcup Y))$. Suppose that $B_{X} \sqcup B \notin \mathcal{B}(M \mid(X \sqcup Y))$. Then there exists $B^{\prime} \subseteq(X \sqcup Y)-B_{X}$ such that $B_{X} \sqcup B \subsetneq B_{X} \sqcup B^{\prime}$ and $\mathrm{B}_{X} \sqcup \mathrm{B}^{\prime} \in \mathcal{J}(M \mid(X \sqcup \mathrm{X}))$. So $\mathrm{B} \subsetneq \mathrm{B}^{\prime} \subseteq \mathrm{Y} \subseteq \mathrm{E}-\mathrm{X}$ and $\mathrm{B}_{\mathrm{X}} \sqcup \mathrm{B}^{\prime} \in \mathcal{J}(M)$, whence $B^{\prime} \in \mathcal{J}(M / X)$, contradicting the choice of $B$. It follows that $B_{X} \sqcup$ $\mathrm{B} \in \mathcal{B}(\mathrm{M} \mid(\mathrm{X} \sqcup \mathrm{Y}))$.

Consequently $\mathcal{J}((M / X) / Y) \subseteq \mathcal{J}(M /(X \sqcup Y))$. Proposition 3.3.7 shows that the anti-containment also holds, and so (2) follows.

We now turn to (3). Clearly $E((M \backslash X) / Y)=E((M / Y) \backslash X)=E-(X \sqcup Y)$. Let $\mathrm{B} \in \mathcal{B}((M \backslash X) \mid Y)$ and let $B_{Y} \in \mathcal{B}(M \mid Y)$. Then

$$
\begin{aligned}
& \mathcal{J}((M \backslash X) / Y)=\{Z \subseteq E-(X \sqcup Y) \mid Z \sqcup B \in \mathcal{J}(M)\}, \text { and } \\
& \mathcal{J}((M / Y) \backslash X)=\left\{Z \subseteq E-(X \sqcup Y) \mid Z \sqcup B_{Y} \in \mathcal{J}(M)\right\} .
\end{aligned}
$$

So it suffices to show that $\mathcal{B}((M \backslash X) \mid Y)=\mathcal{B}(M \mid Y)$. We simply note that

$$
\mathrm{B} \in \mathcal{B}((M \backslash X) \mid Y)
$$

$\Longleftrightarrow B$ is a maximal $\mathcal{J}(M \backslash X)$-subset of $Y$

$\Longleftrightarrow \mathrm{B}$ is a maximal $\mathcal{J}(M)$-subset of $Y \quad$ as $X \cap Y=\varnothing$

$\Longleftrightarrow \mathrm{B} \in \mathcal{B}(M \mid \mathrm{Y})$.

Hence (3) holds.

Corollary 3.6.4. Let $M$ be an independence space and let $X_{1}, \ldots, X_{m}, Y_{1}$, $\ldots, Y_{n} \subseteq \mathrm{E}(\mathrm{M})$ be pairwise disjoint. Then any sequence starting with $\mathrm{M}$ and 
involving the contraction of each $X_{i}$ and the deletion of each $Y_{j}$ has final term $M / \bigsqcup_{i=1}^{m} X_{i} \backslash \bigsqcup_{j=1}^{n} Y_{j}$.

Definition 3.6.5. Let $M$ and $N$ be independence spaces. Then $N$ is a minor of $M$, denoted $N \preccurlyeq M$, if $N=M \backslash X / Y$ for some disjoint $X, Y \subseteq E(M)$.

Proposition 3.6.6. Let $M$ be an independence space and let $X \subseteq E(M)$. Then the circuits of $\mathrm{M} / \mathrm{X}$ are precisely the minimal non-empty elements of $\{\mathrm{C}-\mathrm{X}$ $\mathrm{C} \in \mathcal{C}(\mathrm{M})\}$.

Our argument follows that given on p. 107 of [Ox104].

Proof. The result is trivial if $X$ is empty, so suppose otherwise. Let

$$
y=\{C-X \mid C \in \mathcal{C}(M), C-X \neq \varnothing\}
$$

We establish two claims.

(1) $\mathcal{C}(M / X) \subseteq y$.

Let $C^{\prime} \in \mathcal{C}(M / X)$ and let $B_{X} \in \mathcal{B}(M \mid X)$. Then $C^{\prime} \sqcup B_{X} \notin \mathcal{J}(M)$ and $\left(C^{\prime}-\{e\}\right) \sqcup B_{X} \in \mathcal{J}(M)$ for each $e \in C^{\prime}$. Consequently there exists $\mathrm{C} \in \mathcal{C}(M)$ such that $\mathrm{C}^{\prime} \subseteq \mathrm{C} \subseteq \mathrm{C}^{\prime} \sqcup \mathrm{B}_{\mathrm{X}}$, whence $\mathrm{C}^{\prime}=\mathrm{C}-\mathrm{X}$.

(2) $\left\{Y \in y \mid\left(\forall Y^{\prime} \in y-\{Y\}\right) Y^{\prime} \nsubseteq Y\right\} \subseteq \mathcal{C}(M / X)$.

Let $Y$ be a minimal element of $y$. Then $Y=C-X$ for some $C \in \mathcal{C}(M)$. As $\mathrm{Y}$ is non-empty, $\mathrm{C} \cap \mathrm{X} \subsetneq \mathrm{C}$, so $\mathrm{C} \cap \mathrm{X} \in \mathcal{J}(\mathrm{M} \mid \mathrm{X})$. Consequently there exists $\mathrm{B}_{X} \in \mathcal{B}(M \mid X)$ such that $C \cap X \subseteq B_{X}$. As $C \subseteq C \cup B_{X}$, $C \cup B_{X} \notin \mathcal{J}(M)$, so $Y \notin \mathcal{J}(M / X)$. Thus there exists $C^{\prime} \in \mathcal{C}(M / X)$ contained in $Y$. It follows from (1) that $C^{\prime} \in \mathcal{y}$, so the minimality of $Y$ forces $C^{\prime}=\mathrm{Y}$.

Now let $C^{\prime} \in \mathcal{C}(M / X)$. Then $C^{\prime} \in y$ by (1). Moreover, there exists minimal $Y \in y$ contained in $C^{\prime}$. It follows from (2) that $Y \in \mathcal{C}(M / X)$, so $C^{\prime}=Y$ by (C2). 
Proposition 3.6.7. Let $M$ be an independence space, let $X \subseteq E(M)$ be such that $\mathrm{r}_{\mathrm{M}}(\mathrm{X}) \in \omega$ and let $\mathrm{Y} \subseteq \mathrm{E}-\mathrm{X}$. Then

$$
r_{M / X}(Y)=r_{M}(X \sqcup Y)-r_{M}(X)
$$

Proof. If $r_{M / X}(Y)=\infty$, then $Y$ contains an infinite $\mathcal{J}(M / X)$-subset, so $X \sqcup Y$ contains an infinite $\mathcal{J}(M)$-subset. As $r_{M}(X) \in \omega$, it follows that $r_{M / X}(X \sqcup$ $\mathrm{Y})-\mathrm{r}_{\mathrm{M}}(\mathrm{X})=\infty$.

Otherwise, let $\mathrm{B}_{X} \in \mathcal{B}(M \mid X)$ and let $\mathrm{B} \in \mathcal{B}((M / X) \mid Y)$. Then $\mathrm{B}_{X} \sqcup \mathrm{B} \in$ $\mathcal{B}(M \mid(X \sqcup Y))$, so

$$
\begin{aligned}
r_{M / X}(Y) & =|B| \\
& =\left|B_{X} \sqcup B\right|-\left|B_{X}\right| \\
& =r_{M}(X \sqcup Y)-r_{M}(X) .
\end{aligned}
$$

The next two results respectively characterise the closure operators and flats of contractions of independence spaces.

Proposition 3.6.8. Let $M$ be an independence space, let $X \subseteq E(M)$ and let $\mathrm{Y} \subseteq \mathrm{E}(\mathrm{M})-\mathrm{X}$. Then $\mathrm{cl}_{M / X}(\mathrm{Y})=\mathrm{cl}_{\mathrm{M}}(\mathrm{X} \sqcup \mathrm{Y})-\mathrm{X}$.

Proof. Write $M=(E, \mathcal{J})$, let $B_{X} \in \mathcal{B}(M \mid X)$ and let $B \in \mathcal{B}((M / X) \mid Y)$. Then $\mathrm{B}_{X} \sqcup \mathrm{B} \in \mathcal{B}(M \mid(X \sqcup Y))$, so by (3.5.1),

$$
\begin{aligned}
\mathrm{cl}_{M / X}(\mathrm{Y}) & =\mathrm{cl}_{M / X}(\mathrm{~B}) \\
& =\mathrm{B} \sqcup\{e \in \mathrm{E}-\mathrm{X} \mid \mathrm{B} \sqcup\{e\} \notin \mathcal{J}(\mathrm{M} / \mathrm{X})\} \\
& =\left(\mathrm{B}_{X} \sqcup \mathrm{B} \sqcup\left\{e \in \mathrm{E} \mid \mathrm{B} \mathrm{B}_{\mathrm{X}} \sqcup \mathrm{B} \sqcup\{e\} \notin \mathcal{J}\right\}\right)-\mathrm{X} \\
& =\mathrm{cl}_{M}\left(\mathrm{~B}_{\mathrm{X}} \sqcup \mathrm{B}\right)-\mathrm{X} \\
& =\mathrm{cl}_{M}(\mathrm{X} \sqcup \mathrm{Y})-\mathrm{X} .
\end{aligned}
$$

Corollary 3.6.9. Let $\mathrm{M}$ be an independence space and let $\mathrm{X}, \mathrm{F} \subseteq \mathrm{E}(\mathrm{M})$. Then $\mathrm{F} \in \mathcal{F}(M / X)$ if and only if $\mathrm{X} \sqcup \mathrm{F} \in \mathcal{F}(M)$. 
Proof.

$$
\begin{aligned}
& F \in \mathcal{F}(M / X) \\
\Longleftrightarrow & \mathrm{cl}_{M / X}(F) \subseteq F \subseteq E(M)-X \\
\Longleftrightarrow & c_{M}(X \sqcup F)-X \subseteq F \quad \text { by Prop. 3.6.8 } \\
\Longleftrightarrow & X \sqcup F \in \mathcal{F}(M) .
\end{aligned}
$$

\subsection{Simplification}

Definition 3.7.1. Let $M$ be an independence space on $E$. Define $\rho_{M}: E-$ $\mathcal{L}(M) \rightarrow \mathcal{P}(E-\mathcal{L}(M))$ by

$$
\rho_{M}(e)=\{e\} \sqcup\{f \in E-\mathcal{L}(M) \mid\{e, f\} \text { is a parallel pair of } M\}
$$

for every $e \in E-\mathcal{L}(M)$. Then $\rho_{M}(\cdot)$ induces a relation $\rho_{M}$ on $E$, given by $e \rho_{M} f$ if and only if $e \in \rho_{M}(f)$. The subscript " $M$ " is omitted when there is no ambiguity.

Proposition 3.7.2. Let $M$ be an independence space. Then $\rho_{M}$ is an equivalence relation on $E(M)-\mathcal{L}(M)$.

Proof. Clearly $\rho$ is reflexive and symmetric. So suppose that $e \in \rho(f)$ and $f \in \rho(g)$, where $e, f, g \in E(M)-\mathcal{L}(M)$ are distinct. Then $\{e, f\},\{f, g\} \in$ $\mathcal{C}(M)$, so by Weak Circuit Elimination there exists $C \in \mathcal{C}(M)$ contained in $\{e, g\}$. As $e, g \notin \mathcal{L}(M)$, necessarily $C=\{e, g\}$. Hence $e \in \rho(g)$, and so $\rho$ is transitive.

Definition 3.7.3. Let $M$ be an independence space. Then a parallel class of $M$ is a $\rho_{M}$-equivalence class.

Recall that a transversal of a partition $\left\{Y_{i} \mid i \in I\right\}$ of a set $Y$ is a set $X \subseteq Y$ meeting each $X_{i}$ at precisely one element. 
Definition 3.7.4. Let $M$ be an independence space. Then the simplification of $M$, denoted $\widetilde{M}$, is $M \mid X$ where $X$ is a transversal of the parallel classes of $M$.

Clearly simplifications are simple, and an independence space $M$ is simple if and only if $M=\widetilde{M}$ for some $\widetilde{M}$.

Definition 3.7.5. Let $M=(E, \mathcal{J})$ and $M^{\prime}=\left(E^{\prime}, J^{\prime}\right)$ be independence spaces. Then $M$ and $M^{\prime}$ are isomorphic, denoted $M \cong M^{\prime}$, if there exists a bijection $\chi: E \rightarrow E^{\prime}$ such that $X \in \mathcal{J}$ if and only if $\chi[X] \in \mathcal{J}^{\prime}$ for every $X \subseteq E$.

Proposition 3.7.6. Isomorphism of independence spaces is an equivalence relation.

We omit the easy proof of Proposition 3.7.6.

Proposition 3.7.7. The simplification of an independence space is defined uniquely up to isomorphism.

Proof. Let $M$ be an independence space on $E$ and let $X, X^{\prime} \subseteq E$ determine two simplifications of $M$. Let $\left\{\rho_{i} \mid i \in I\right\}$ be the collection of parallel classes of $M$. For every $i \in I$, let $x_{i} \in \rho_{i} \cap X$ and let $x_{i}^{\prime} \in \rho_{i} \cap X^{\prime}$. Define $\chi: X \rightarrow X^{\prime}$ by $\chi\left(x_{i}\right)=x_{i}^{\prime}$ for every $i \in I$. Clearly $\chi$ is a bijection. Moreover, for every $Y \subseteq X$,

$$
\mathrm{Y} \in \mathcal{J}(M \mid X) \Longleftrightarrow \mathrm{Y} \in \mathcal{J}(M) \Longleftrightarrow \chi[Y] \in \mathcal{J}(M) \Longleftrightarrow \chi[Y] \in \mathcal{J}\left(M \mid X^{\prime}\right)
$$

Hence $M|X \cong M| X^{\prime}$.

\subsection{Geometric Representations}

Geometric representations provide a useful means of visualising matroids of rank at most 4 . A geometric representation of a matroid $M$ having rank at most 4 is a diagram having the following properties. 
(1) Each element $e$ of $M$ is represented by a single point, which is labelled by $e$.

(2) All loops of $M$ are placed in a box to one side of the diagram.

(3) Elements in the same parallel class are represented by a single point, which is labelled by the elements of the parallel class.

(4) Elements of a triangle are collinear.

(5) Elements of a 4-circuit are coplanar.

For example, consider the geometric representation given in Figure 3.2. The matroid depicted has ground set $\{a, b, c, d, e, f, g, h\}$. The elements $g$ and $h$ are loops, and $\{e, f\}$ is a parallel pair. The set $\{a, b, c\}$ is a circuit, as is $\{a, b, d, e\}$. As every set of four points is coplanar, this matroid has rank at most 3. Moreover, $\{a, b, d\}$ is independent, so the matroid in fact has rank 3. Its flats include $\{a, g, h\},\{a, b, c, g, h\}$ and $\{b, d, g, h\}$.

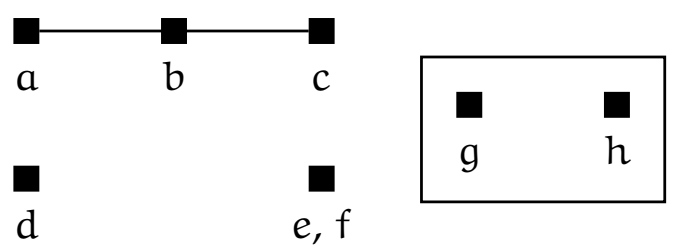

Figure 3.2: A geometric representation of a rank-3 matroid.

Geometric representations of matroids having rank 4 are viewed 3-dimensionally, reflecting the representation of each basis by a set of 4 noncoplanar points. For example, consider the geometric representation of the Escher matroid given in Figure 3.3. This is intended to be viewed such that the convex hull of the points shown is a tetrahedron in $\mathbb{R}^{3}$.

As the Escher matroid has no loops, the box for loops has been omitted from Figure 3.3. We follow the convention that when this box is omitted, 


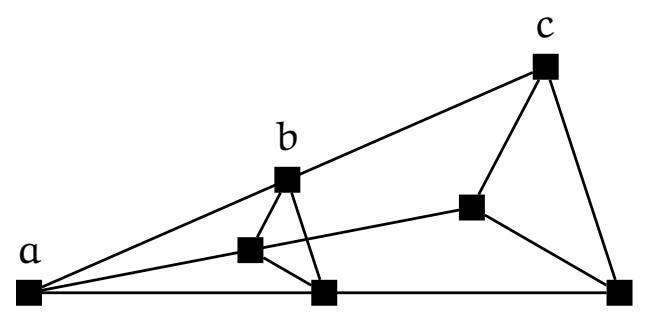

Figure 3.3: A geometric representation of the Escher matroid.

it is to be understood that there are no loops. Similarly, when labels are omitted, it is to be understood that there are no non-trivial parallel classes (so the Escher matroid has no parallel pairs).

The Escher matroid demonstrates an important property of these diagrams: not every diagram of this type is a geometric representation of a matroid. For example, suppose that Figure 3.3 is redrawn so that the points $a, b$ and $c$ no longer colline, but no other collineations are altered. Then the resulting diagram is not a geometric representation of a matroid, for if it were, then the dependencies shown, together with the matroid axioms and rule (4) for geometric representations would force $a, b$ and $c$ to colline.

Further discussion of geometric representations is given on pp. $36-46$ of [Ox104].

\subsection{Connectivity}

In general, the connectivity between two subobjects $X^{\prime}$ and $Y^{\prime}$ of an object $Z$ (such as a graph, independence space or point configuration) is the least order of a separation of $X^{\prime}$ and $Y^{\prime}$ in $Z$. This section examines connectivity in independence spaces, which is similar to connectivity in graphs. To motivate the definition given, we first comment on connectivity in graphs. 
Let $G$ be a graph having edge set $E$ and let $X^{\prime}, Y^{\prime} \subseteq E$. Note that $X^{\prime} \cap Y^{\prime}$ may be non-empty. Then the vertex connectivity between $X^{\prime}$ and $Y^{\prime}$ in $G$, denoted $\kappa_{G}\left(X^{\prime}, Y^{\prime}\right)$, is

$$
\mathrm{K}_{\mathrm{G}}\left(\mathrm{X}^{\prime}, \mathrm{Y}^{\prime}\right)=\inf \left\{\left|\mathrm{V}_{\mathrm{G}}(\mathrm{X}) \cap \mathrm{V}_{\mathrm{G}}(\mathrm{Y})\right|: \mathrm{X}^{\prime} \subseteq X, \mathrm{Y}^{\prime} \subseteq \mathrm{Y}, \mathrm{X} \cup \mathrm{Y}=\mathrm{E}\right\}
$$

This is easily visualised, as indicated in Figure 3.4.

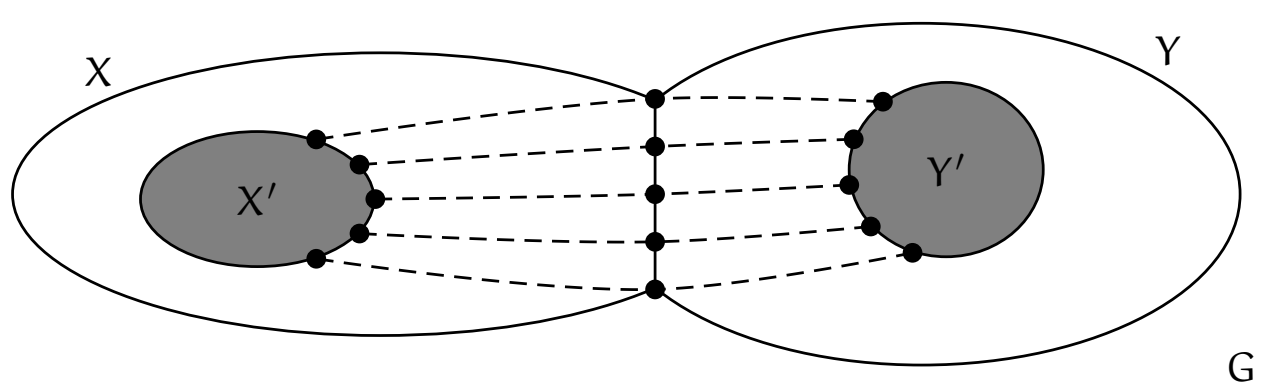

Figure 3.4: A graph $G$ having $\kappa_{G}\left(X^{\prime}, Y^{\prime}\right)=5$. This connectivity is concretely realised as both a vertex cut $\mathrm{V}(\mathrm{X}) \cap \mathrm{V}(\mathrm{Y})$ and a set of vertex-disjoint $X^{\prime}-Y^{\prime}$ paths, each of size 5 .

Any set of paths associated with a minimum-order vertex cut as in Figure 3.4 may be thought of as representing "units of dependency" between $X^{\prime}$ and $Y^{\prime}$ in $G$. In an independence space, dependencies correspond to circuits. Given a matroid $M$, a circuit $C \in \mathcal{C}(M)$ and $X, Y \in \mathcal{F}(M)$ such that $X \cup Y=E(M)$ and $X \nsupseteq C \nsubseteq Y$, it is possible for $C \cap X \cap Y$ to be empty, as shown in Figure 3.5.

In the example of Figure 3.5, the connectivity between $X$ and $Y$ should be 1 . If $X \cap Y$ contained a non-loop element, the value 1 could be obtained as $r(X \cap Y)$-an analogue of the size of a minimum-order vertex cut in a graph. However, there is no such element (unlike the graph case, in which vertices are always present). This difficulty can be negotiated by taking a difference of ranks, as in the following definition. 
Definition 3.9.1. Let $M$ be a finite-rank independence space on $E$. Define $\lambda_{M}:\{(X, Y) \mid X \cup Y=E\} \rightarrow \omega$ by

$$
\lambda_{M}(X, Y)=r_{M}(X)+r_{M}(Y)-r(M)
$$

whenever $X, Y \subseteq E$ are such that $X \cup Y=E$, and define $\lambda_{M}: \mathcal{P}(E) \rightarrow \omega$ by

$$
\lambda_{M}(X)=\lambda_{M}(X, E-X)
$$

for every $X \subseteq E$. Then define $\kappa_{M}: \mathcal{P}(E) \times \mathcal{P}(E) \rightarrow \omega \sqcup\{\infty\}$ by

$$
\mathrm{K}_{M}\left(X^{\prime}, Y^{\prime}\right)=\inf \left\{\lambda_{M}(X, Y) \mid X^{\prime} \subseteq X, Y^{\prime} \subseteq Y, X \cup Y=E\right\}
$$

for every $X^{\prime}, Y^{\prime} \subseteq E$. The subscript " $M$ " is omitted when there is no ambiguity. Note that some authors define $\lambda_{M}(X, Y)$ to be $r_{M}(X)+r_{M}(Y)-$ $r(M)+1$.

The following proposition notes some elementary properties of the connectivity functions $\lambda$ and $\kappa$.

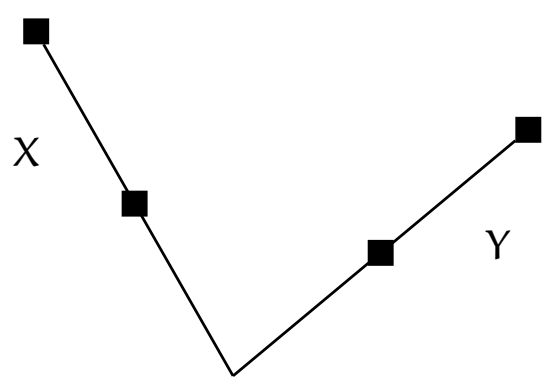

Figure 3.5: A geometric representation of a 4-circuit, which is a union of two proper flats $X$ and $Y$ having empty intersection.

Proposition 3.9.2. For every finite-rank independence space,

(1) $\lambda(\cdot, \cdot), \lambda(\cdot)$ and $\mathrm{\kappa}(\cdot, \cdot)$ are each symmetric, and 
(2) $\mathrm{k}(\cdot, \cdot)$ is increasing in each of its arguments.

The next proposition follows readily from equality of the ranks of a set and its closure.

Proposition 3.9.3. For every finite-rank independence space on $\mathrm{E}$,

(1) $\lambda(X, Y)=\lambda(\mathrm{cl}(\mathrm{X}), \mathrm{Y})$ whenever $\mathrm{X}, \mathrm{Y} \subseteq \mathrm{E}$ are such that $\mathrm{X} \cup \mathrm{Y}=\mathrm{E}$,

(2) $\lambda(X)=\lambda(\mathrm{cl}(\mathrm{X}))$ for every $\mathrm{X} \subseteq \mathrm{E}$, and

(3) $\mathrm{k}(\mathrm{X}, \mathrm{Y})=\mathrm{k}(\mathrm{cl}(\mathrm{X}), \mathrm{Y})$ for every $\mathrm{X}, \mathrm{Y} \subseteq \mathrm{E}$.

Proof. Clearly it suffices to demonstrate (1). So let $M$ be a finite-rank independence space on $E$ and let $X, Y \subseteq E$ be such that $X \cup Y=E$. Then

$$
\begin{aligned}
\lambda(X, Y) & =r(X)+r(Y)-r(M) \\
& =r(c l(X))+r(Y)-r(M) \quad \text { by Lem. 3.5.4 } \\
& =\lambda(c l(X), Y) .
\end{aligned}
$$

The result follows.

We close this section by observing a result that is well known for matroids, namely that the connectivity function $\lambda_{M}$ of a finite-rank independence space $M$ inherits submodularity from the rank function $r_{M}$.

Proposition 3.9.4. $\lambda(\cdot)$ is submodular for every finite-rank independence space. Proof. Let $M$ be a finite-rank independence space on $E$ and let $X, Y \subseteq E$. Then

$$
\begin{aligned}
\lambda(X)+\lambda(Y)= & r(X)+r(E-X)+r(Y)+r(E-Y)-2 r(M) \\
\geqslant & r(X \cap Y)+r(X \cup Y)+r(E-(X \cup Y)) \\
& +r(E-(X \cap Y))-2 r(M) \\
= & \lambda(X \cap Y)+\lambda(X \cup Y) .
\end{aligned}
$$




\section{Chapter 4}

\section{Configurations}

The term "configuration" is used in many different, usually geometric, contexts in mathematics. The configurations examined in this thesis are sometimes called point configurations to distinguish them from other types of configuration. As point configurations are the only type of configuration concerning us, we hereafter write "configuration" to mean "point configuration."

Our focus is predominantly on the matroidal structure of configurations. This is reflected in the purely matroidal formulation of many results, those of Chapters 3 and 5 in particular.

The main advantages of working with configurations instead of independence spaces in general are

- the ability to harness the methods of linear algebra for arguments, and

- the ability to (sometimes implicitly) use any points in the ambient vector space.

This chapter is devoted to developing configuration-specific formulations of ideas introduced for independence spaces in Chapter 3. The presence of a vector space embedding introduces some minor technical- 
ities, notably the requirement that some definitions be made only up to an equivalence of configurations.

\subsection{Definitions}

Definition 4.1.1. Let $V$ be a vector space. Then a $V$-configuration is a triple $\mathcal{D}=(D, E, \ell)$, where $E$ and $D \subseteq V$ are sets and $\ell: E \rightarrow D$ is a surjective function. We shall often write "DD is a configuration" to mean that $\mathcal{D}$ is a $\mathrm{V}$-configuration for some vector space $\mathrm{V}$.

Given a $V$-configuration $\mathcal{D}=(D, E, \ell)$, the point set of $\mathcal{D}$ is $D$, while the ground set of $\mathcal{D}$, denoted $E(\mathcal{D})$, is $E$. The order of $\mathcal{D}$, denoted $|\mathcal{D}|$, is $|D|$, and the dimension of $\mathcal{D}$, denoted $\operatorname{dim}(\mathcal{D})$, is $\operatorname{dim}(\langle\mathrm{D}\rangle)$. A configuration is finite if its ground set is finite.

Clearly $|\mathcal{D}| \leqslant|E(\mathcal{D})|$ for every configuration $\mathcal{D}$. In particular, every finite configuration has finite order. As a point may be labelled by two distinct elements of the ground set, it is possible for this inequality to be strict.

The following classical proposition establishes that configurations give rise to independence spaces, justifying the earlier use of the phrase "represented independence space."

Proposition 4.1.2. Let $\mathcal{D}=(\mathrm{D}, \mathrm{E}, \ell)$ be a configuration. Define $\mathcal{J} \subseteq \mathcal{P}(\mathrm{E})$ by $\mathrm{X} \in \mathcal{J}$ if and only if $\left.\ell\right|_{X}$ is injective and $\ell[X]$ is linearly independent. Then $(E, J)$ is an independence space.

For completeness, we reproduce the argument given on p. 8 of [Ox104].

Proof. The statements (I1) and (I2) are clear. Finiteness of linear combinations gives (I4), so we demonstrate (I3).

Let $I_{1}, I_{2} \in \mathcal{J}$ be such that $\left|I_{1}\right|<\left|I_{2}\right| \in \omega$ and let $V=\left\langle\ell\left[I_{1} \cup I_{2}\right]\right\rangle$. Then $\operatorname{dim}(V) \geqslant \operatorname{dim}\left(\left\langle\ell\left[I_{2}\right]\right\rangle\right)=\left|I_{2}\right|$. Suppose that $I_{1} \sqcup\{e\} \notin \mathcal{J}$ for every $e \in I_{2}-I_{1}$. 
Then $\mathrm{V} \subseteq\left\langle\ell\left[\mathrm{I}_{1}\right]\right\rangle$, so

$$
\left|\mathrm{I}_{2}\right| \leqslant \operatorname{dim}(\mathrm{V}) \leqslant \operatorname{dim}\left(\left\langle\ell\left[\mathrm{I}_{1}\right]\right\rangle\right)=\left|\mathrm{I}_{1}\right|<\left|\mathrm{I}_{2}\right|
$$

- a contradiction. Hence there exists $e \in \mathrm{I}_{2}-\mathrm{I}_{1}$ such that $\mathrm{I}_{1} \sqcup\{e\} \in \mathcal{J}$, and so (I3) holds.

It is now possible to make the following formal definition.

Definition 4.1.3. Let $\mathcal{D}$ be a configuration. Then the vector independence space of $\mathcal{D}$, denoted $M[\mathcal{D}]$, is the independence space derived from $\mathcal{D}$ via Proposition 4.1.2. An independence space $M$ is represented by a configuration $\mathcal{D}$ if $M=M[\mathcal{D}]$.

The dependent sets of $\mathcal{D}$ are precisely the dependent sets of $M[\mathcal{D}]$. The notions of independent set, circuit, basis, loop, parallel pair, triangle and flat are then inherited from independence spaces (see pp. 18 and 33 for definitions of these concepts for independence spaces). The collections of independent sets, circuits, bases, loops and flats of $\mathcal{D}$ are denoted by $\mathcal{J}(\mathcal{D}), \mathcal{C}(\mathcal{D}), \mathcal{B}(\mathcal{D}), \mathcal{L}(\mathcal{D})$ and $\mathcal{F}(\mathcal{D})$ respectively.

A simple graph can always be obtained from a graph by deleting all loops and then identifying all edges incident on the same pair of vertices. This idea does not transfer directly to configurations: identifying all labels of a point may still leave non-trivial parallel classes, for two distinct nonzero points may be linearly dependent.

Example 4.1.4. Let $\mathcal{D}_{1}$ and $\mathcal{D}_{2}$ be the $\mathbb{R}^{2}$-configurations on ground set $\{a, b, c, d\}$ given by

$$
\ell_{1}(a)=\ell_{1}(b)=\ell_{1}(c)=(2,0), \ell_{1}(d)=(0,1)
$$

and

$$
\ell_{2}(a)=(1,0), \ell_{2}(b)=(2,0), \ell_{2}(c)=(3,0), \ell_{2}(d)=(0,1)
$$

respectively. These are illustrated in Figure 4.1. 

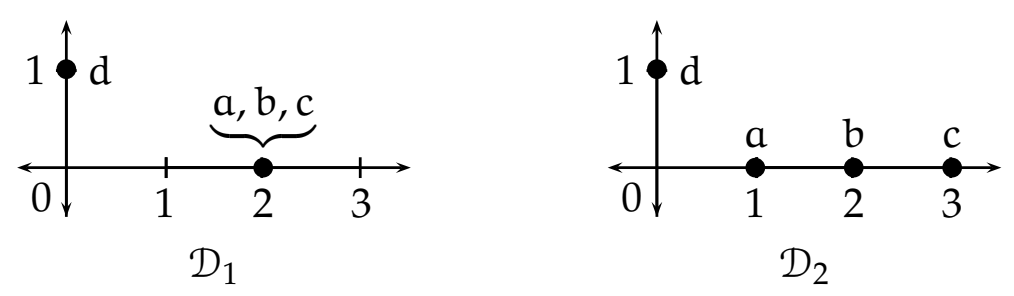

Figure 4.1: The configurations $\mathcal{D}_{1}$ and $\mathcal{D}_{2}$ of Example 4.1.4.

Both $\mathcal{D}_{1}$ and $\mathcal{D}_{2}$ consist of a parallel class of size 3 (i.e., $\{a, b, c\}$ ) and a non-loop point (i.e., d), from which it is clear that they have the same matroidal structure (i.e., $M\left[\mathcal{D}_{1}\right]=M\left[\mathcal{D}_{2}\right]$ ). Despite this, identifying the labels of each point in each configuration eliminates the parallel class $\{a, b, c\}$ from $\mathcal{D}_{1}$, yet leaves it unchanged in $\mathcal{D}_{2}$.

\subsection{Equivalence}

Example 4.1.4 shows that different configurations may share the same matroidal structure. In particular, configurations only differing "within" parallel classes are matroidally indistinguishable. It is convenient to identify such configurations, so we introduce the following definition.

Definition 4.2.1. Let $\mathcal{D}=(D, E, \ell)$ and $\mathcal{D}^{\prime}=\left(D^{\prime}, E^{\prime}, \ell^{\prime}\right)$ be configurations. Then $\mathcal{D}$ and $\mathcal{D}^{\prime}$ are equivalent if there exist an invertible linear transformation $\phi:\langle\mathrm{D}\rangle \rightarrow\left\langle\mathrm{D}^{\prime}\right\rangle$ and a bijection $\chi: E \rightarrow \mathrm{E}^{\prime}$ such that $\langle\phi(\ell(e))\rangle=$ $\left\langle\ell^{\prime}(\chi(e))\right\rangle$ for every $e \in E$. In this case, we say that $(\phi, \chi)$ is an equivalence of $\mathcal{D}$ and $\mathcal{D}^{\prime}$, denoted by $(\phi, \chi): \mathcal{D} \rightarrow \mathcal{D}^{\prime}$ or $\mathcal{D} \stackrel{(\phi, \chi)}{\longrightarrow} \mathcal{D}^{\prime}$.

Proposition 4.2.2. Equivalence of configurations is an equivalence relation.

Proof. We omit functional parentheses from this argument for clarity. Let $\mathcal{D}=(D, E, \ell) \stackrel{(\phi, x)}{\longrightarrow} \mathcal{D}^{\prime}=\left(D^{\prime}, E^{\prime}, \ell^{\prime}\right) \stackrel{\left(\phi^{\prime}, \chi^{\prime}\right)}{\longrightarrow} \mathcal{D}^{\prime \prime}=\left(D^{\prime \prime}, E^{\prime \prime}, \ell^{\prime \prime}\right)$. Then for every $e \in E$,

$$
\left\langle\operatorname{id}_{\langle D} \ell e\right\rangle=\langle\ell e\rangle=\left\langle\ell \operatorname{id}_{E} e\right\rangle .
$$


Thus $\left(\mathrm{id}_{\langle\mathrm{D}\rangle}, \mathrm{id}_{\mathrm{E}}\right): \mathcal{D} \rightarrow \mathcal{D}$, and so reflexivity holds. Moreover, for every $e^{\prime} \in E^{\prime}$,

$$
\begin{aligned}
\left\langle\ell^{\prime} e^{\prime}\right\rangle=\left\langle\ell^{\prime} \chi \chi^{-1} e^{\prime}\right\rangle & =\left\langle\phi \ell \chi^{-1} e^{\prime}\right\rangle \\
\phi^{-1}\left[\left\langle\ell^{\prime} e^{\prime}\right\rangle\right] & =\phi^{-1}\left[\left\langle\phi \ell \chi^{-1} e^{\prime}\right\rangle\right] \\
\left\langle\phi^{-1} \ell^{\prime} e^{\prime}\right\rangle & =\left\langle\ell \chi^{-1} e^{\prime}\right\rangle .
\end{aligned}
$$

Thus $\left(\phi^{-1}, \chi^{-1}\right): \mathcal{D}^{\prime} \rightarrow \mathcal{D}$, and so symmetry holds. Finally, for every $e \in E$,

$$
\begin{aligned}
\langle\phi \ell e\rangle & =\left\langle\ell^{\prime} \chi e\right\rangle \\
\phi^{\prime}[\langle\phi \ell e\rangle] & =\phi^{\prime}\left[\left\langle\ell^{\prime} \chi e\right\rangle\right] \\
\left\langle\left(\phi^{\prime} \circ \phi\right) \ell e\right\rangle & =\left\langle\phi^{\prime} \ell^{\prime} \chi e\right\rangle=\left\langle\ell^{\prime \prime}\left(\chi^{\prime} \circ \chi\right) e\right\rangle .
\end{aligned}
$$

Hence $\left(\phi^{\prime} \circ \phi, \chi^{\prime} \circ \chi\right): \mathcal{D} \rightarrow \mathcal{D}^{\prime \prime}$, and so transitivity holds.

Proposition 4.2.3. Equivalent configurations have isomorphic vector independence spaces.

Proof. Let $(\phi, \chi): \mathcal{D}=(D, E, \ell) \rightarrow \mathcal{D}^{\prime}=\left(D^{\prime}, E^{\prime}, \ell^{\prime}\right)$ be an equivalence of configurations, and define a set of lines in a vector space to be independent if none is contained in the span of the others. Then

$$
X \in \mathcal{J}(M[\mathcal{D}])
$$

$\left.\Longleftrightarrow \ell\right|_{X}$ is injective and $\ell[X]$ is linearly independent

$\Longleftrightarrow \psi: X \rightarrow \mathcal{S}(\langle D\rangle): x \mapsto\langle\ell(x)\rangle$ is injective and $\operatorname{im}(\psi)$ is independent

$\Longleftrightarrow \psi: X \rightarrow \mathcal{S}\left(\left\langle D^{\prime}\right\rangle\right): x \mapsto\langle(\phi \circ \ell)(x)\rangle$ is injective and $\operatorname{im}(\psi)$ is independent as $\phi$ is bijective and linear

$\Longleftrightarrow \psi: X \rightarrow \mathcal{S}\left(\left\langle D^{\prime}\right\rangle\right): x \mapsto\left\langle\left(\ell^{\prime} \circ \chi\right)(x)\right\rangle$ is injective and $\operatorname{im}(\psi)$ is independent by equivalence

$\left.\Longleftrightarrow \ell^{\prime}\right|_{\chi[X]}$ is injective and $\ell^{\prime}[\chi[X]]$ is linearly independent 
$\Longleftrightarrow \chi[X] \in \mathcal{J}\left(M\left[\mathcal{D}^{\prime}\right]\right)$

Hence $M[\mathcal{D}] \cong M\left[\mathcal{D}^{\prime}\right]$.

The converse of Proposition 4.2.3 does not hold, as configurations differing "between" parallel classes may be inequivalent but nonetheless have isomorphic vector independence spaces. For example, consider Figure 4.2. Each of the configurations shown has 8 elements and a collection of circuits consisting of a pair of disjoint triangles and all 4-element sets containing neither triangle. Consequently the configurations have isomorphic vector matroids. However, as linear transformations preserve incidence, there is no linear transformation mapping the triangles of the first configuration onto those of the second that also maps the remaining pair of points in the first configuration onto those of the second. Thus the configurations are inequivalent.
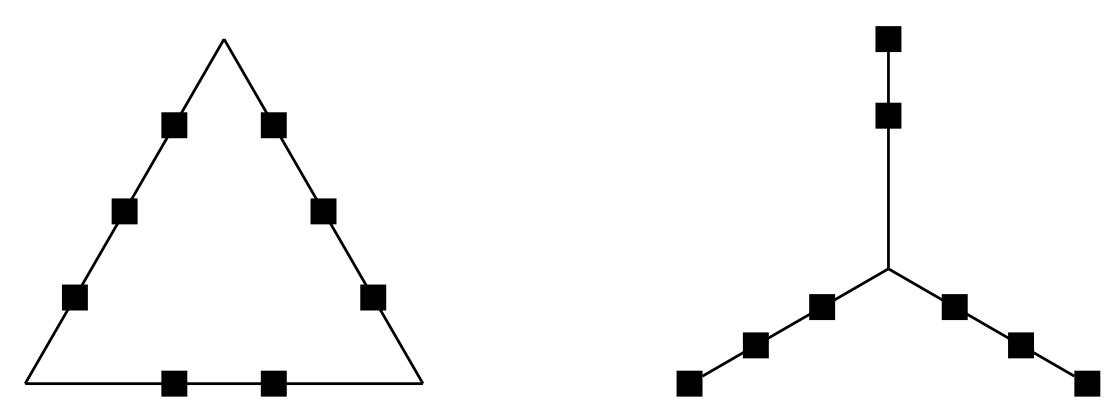

Figure 4.2: Geometric representations of a pair of inequivalent configurations having isomorphic vector matroids.

The following definition is the first of several made only up to an equivalence of configurations.

Definition 4.2.4. Let $\mathcal{D}$ be a configuration having point set $\mathrm{D}$. Then the simplification of $\mathcal{D}$, denoted $\widetilde{\mathcal{D}}$, is $\left(\widetilde{\mathrm{D}}, \widetilde{\mathrm{D}}, \mathrm{id}_{\widetilde{\mathrm{D}}}\right)$ where $\widetilde{\mathrm{D}}$ is a transversal of

$$
\{\langle d\rangle \cap(D-\{0\}) \mid d \in D-\{0\}\}
$$


We say that $\mathcal{D}$ is simple if $\mathcal{D}=\widetilde{\mathcal{D}}$ for some $\widetilde{\mathcal{D}}$.

Proposition 4.2.5. The simplification of a configuration is defined uniquely up to an equivalence of configurations.

Proof. Let $\widetilde{\mathcal{D}}_{1}=\left(\widetilde{\mathrm{D}}_{1}, \widetilde{\mathrm{D}}_{1}, \mathrm{id}_{\widetilde{\mathrm{D}}_{1}}\right)$ and $\widetilde{\mathcal{D}}_{2}=\left(\widetilde{\mathrm{D}}_{2}, \widetilde{\mathrm{D}}_{2}, \mathrm{id}_{\widetilde{\mathrm{D}}_{2}}\right)$ be simplifications of a configuration $\mathcal{D}$ having point set $D$. Write

$$
\{\langle d\rangle \cap(D-\{\mathbf{0}\}) \mid d \in D-\{\mathbf{0}\}\}=\left\{\rho_{i} \mid i \in I\right\}
$$

Let $\phi=\mathrm{id}_{\langle\mathrm{D}\rangle}$ and define $\chi: \widetilde{\mathrm{D}}_{1} \rightarrow \widetilde{\mathrm{D}}_{2}$ by $\chi\left(\mathrm{d}_{1 i}\right)=\mathrm{d}_{2 i}$ for every $i \in \mathrm{I}$, where $\rho_{i} \cap \widetilde{D}_{j}=\left\{d_{j i}\right\}$ for every $i \in I$ and each $j=1$, 2. Then for every $\mathrm{d} \in \widetilde{\mathrm{D}}_{1}$

$$
\begin{aligned}
\left\langle\phi\left(\operatorname{id}_{\widetilde{D}_{1}}(\mathrm{~d})\right)\right\rangle & =\langle\phi(\mathrm{d})\rangle \\
& =\langle\mathrm{d}\rangle \\
& =\langle\chi(\mathrm{d})\rangle \\
& =\left\langle\mathrm{id}_{\widetilde{\mathrm{D}}_{2}}(\chi(\mathrm{d}))\right\rangle .
\end{aligned}
$$

Hence $(\phi, \chi): \widetilde{\mathcal{D}}_{1} \rightarrow \widetilde{\mathcal{D}}_{2}$.

The next proposition verifies that simplification in configurations corresponds to simplification in independence spaces.

Proposition 4.2.6. Let $\mathcal{D}$ be a configuration. Then $\widetilde{M[\mathcal{D}]} \cong M[\widetilde{\mathcal{D}}]$.

Proof. Let $\mathcal{D}=(\mathrm{D}, \mathrm{E}, \ell)$ be a configuration. Then $\widetilde{\mathcal{D}}=\left(\widetilde{\mathrm{D}}, \widetilde{\mathrm{D}}, \mathrm{id}_{\widetilde{\mathrm{D}}}\right)$, where $\widetilde{\mathrm{D}}$ is a transversal of

$$
\{\langle\mathrm{d}\rangle \cap(\mathrm{D}-\{\mathbf{0}\}) \mid \mathrm{d} \in \mathrm{D}-\{\mathbf{0}\}\}
$$

and $M[\widetilde{\mathcal{D}}]=(\widetilde{\mathrm{D}}, \mathcal{J})$, where

$$
\mathcal{J}=\{\mathrm{Y} \subseteq \widetilde{\mathrm{D}} \mid \mathrm{Y} \text { is linearly independent }\} .
$$


Moreover, $M[\mathcal{D}]=\left(E, J^{\prime}\right)$, where

$\mathcal{J}^{\prime}=\left\{\mathrm{Y} \subseteq \mathrm{E}:\left.\ell\right|_{\mathrm{Y}}\right.$ is injective and $\ell[\mathrm{Y}]$ is linearly independent $\}$

Let $\chi$ be a choice function for $\left\{\ell^{-1}\{d\} \mid d \in \widetilde{D}\right\}$. Then $X:=\operatorname{im}(\chi)$ is a transversal of the parallel classes of $M[\mathcal{D}]$. Consequently $\widehat{M[D}] \cong$ $(\mathrm{M}[\mathcal{D}]) \mid \mathrm{X}$ by Proposition 3.7.7. Moreover,

$$
Y \in \mathcal{J}
$$

$\Longleftrightarrow \mathrm{Y}$ is linearly independent

$\left.\Longleftrightarrow \ell\right|_{X[Y]}$ is injective and $\ell[X[Y]]$ is linearly independent $\Longleftrightarrow \chi[Y] \in \mathcal{J}^{\prime}$.

Hence $M[\widetilde{\mathcal{D}}] \cong(M[\mathcal{D}]) \mid X$, so $\widetilde{M[\mathcal{D}]} \cong M[\widetilde{\mathcal{D}}]$ by Proposition 3.7.6.

We close this section by deriving a result for configurations from Lemma 3.4.9. This will later provide the width bound required in Theorem 6.2.5.

Proposition 4.2.7. Let $\mathcal{D}=(\mathrm{D}, \mathrm{E}, \ell)$ be a finite-dimensional configuration and let $\mathrm{E}=\mathrm{E}_{1} \sqcup \mathrm{E}_{2} \sqcup \mathrm{E}_{3}$. Let $\mathrm{k} \in \mathrm{\omega}$ and suppose that

$$
\operatorname{dim}\left(\left\langle\ell\left[E_{i}\right]\right\rangle\right)+\operatorname{dim}\left(\left\langle\ell\left[E-E_{i}\right]\right\rangle\right) \leqslant \operatorname{dim}(\mathcal{D})+k
$$

for each $i=1,2,3$. Then

$$
\sum_{i=1}^{3} \operatorname{dim}\left(\left\langle\ell\left[E-E_{i}\right]\right\rangle\right)-2 \operatorname{dim}(\mathcal{D}) \leqslant 2 k .
$$

Proof. We simply observe that for each $i$,

$$
r_{M}\left(E_{i}\right)+r_{M}\left(E-E_{i}\right) \leqslant r(M)+k
$$


where $M:=M[\mathcal{D}]$. It then follows from Lemma 3.4.9 that

$$
\begin{aligned}
2 k & \geqslant \sum_{i=1}^{3} r_{M}\left(E-E_{i}\right)-2 r(M) \\
& =\sum_{i=1}^{3} \operatorname{dim}\left(\left\langle\ell\left[E-E_{i}\right]\right\rangle\right)-2 \operatorname{dim}(\mathcal{D}) .
\end{aligned}
$$

\subsection{Restriction}

This brief section is devoted to formally defining some elementary concepts encountered frequently later.

Definition 4.3.1. Let $\mathcal{D}=(D, E, \ell)$ be a configuration and let $X \subseteq E$. Then the restriction of $\mathcal{D}$ to $X$, denoted $\mathcal{D} \mid X$, is $\left(\ell[X], X,\left.\ell\right|_{X}\right)$. The deletion of $X$ from $\mathcal{D}$, denoted $\mathcal{D} \backslash X$, is $\mathcal{D} \mid(E-X)$.

The following definition makes use of the formal set-theoretical definition of the functions $\ell_{i}$.

Definition 4.3.2. Let $\left\{\mathcal{D}_{i}=\left(D_{i}, E_{i}, \ell_{i}\right) \mid i \in I\right\}$ be a collection of $V$-configurations such that $\ell_{i}\left|\left(E_{i} \cap E_{j}\right)=\ell_{j}\right|\left(E_{i} \cap E_{j}\right)$ for every $i, j \in I$. Then the union of $\left\{\mathcal{D}_{i} \mid i \in I\right\}$, denoted $\bigcup_{i \in I} \mathcal{D}_{i}$, is $\left(\bigcup_{i \in I} D_{i}, \bigcup_{i \in I} E_{i}, \bigcup_{i \in I} \ell_{i}\right)$.

Definition 4.3.3. Let $\left\{\mathcal{D}_{i}=\left(D_{i}, E_{i}, \ell_{i}\right) \mid i \in I\right\}$ be a collection of $V$-configurations such that $\ell_{j}\left|\bigcap_{i \in I} E_{i}=\ell_{k}\right| \bigcap_{i \in I} E_{i}$ for every $j, k \in I$. Then the intersection of $\left\{\mathcal{D}_{i} \mid i \in I\right\}$, denoted $\bigcap_{i \in I} \mathcal{D}_{i}$, is $\mathcal{D}_{j} \mid \bigcap_{i \in I} E_{i}$, where $j \in I$ is arbitrary.

Clearly

- the class of V-configurations is closed under the restriction, deletion, union and intersection operations, and

- every intersection of V-configurations is independent of the choice of the index $j$. 
As the class of V-configurations is closed under restriction, it is sensible to define a notion of subconfiguration.

Definition 4.3.4. Let $\mathcal{D}$ and $\mathcal{D}^{\prime}$ be configurations. Then $\mathcal{D}^{\prime}$ is a subconfiguration of $\mathcal{D}$, denoted $\mathcal{D}^{\prime} \leqslant \mathcal{D}$, if $\mathcal{D}^{\prime}=\mathcal{D} \mid X$ for some $X \subseteq \mathrm{E}(\mathcal{D})$.

Proposition 4.3.5. The subconfiguration relation $\leqslant$ is a partial order on the set of all subconfigurations of a given configuration.

Proof. Let $\mathcal{D}_{i}=\left(D_{i}, E_{i}, \ell_{i}\right) \leqslant \mathcal{D}$ for each $i=1,2,3$. Clearly $\leqslant$ is reflexive, so we consider symmetry and transitivity.

Suppose that $\mathcal{D}_{1} \leqslant \mathcal{D}_{2} \leqslant \mathcal{D}_{1}$. Then $\mathrm{E}_{1} \subseteq \mathrm{E}_{2} \subseteq \mathrm{E}_{1}$, so $\mathrm{E}_{1}=\mathrm{E}_{2}$. Consequently $\ell_{2}=\left.\ell_{1}\right|_{E_{2}}=\left.\ell_{1}\right|_{E_{1}}=\ell_{1}$. Thus $\mathcal{D}_{1}=\mathcal{D}_{2}$, and so $\leqslant$ is symmetric.

Now suppose that $\mathcal{D}_{1} \leqslant \mathcal{D}_{2} \leqslant \mathcal{D}_{3}$. Then $\mathrm{E}_{1} \subseteq \mathrm{E}_{2} \subseteq \mathrm{E}_{3}$, so $\mathrm{E}_{1} \subseteq \mathrm{E}_{3}$. Consequently $\ell_{1}=\left.\ell_{2}\right|_{\mathrm{E}_{1}}=\left.\left(\ell_{3} \mid \mathrm{E}_{2}\right)\right|_{\mathrm{E}_{1}}=\left.\ell_{3}\right|_{\mathrm{E}_{1}}$. Hence $\mathcal{D}_{1} \leqslant \mathcal{D}_{3}$, and so $\leqslant$ is transitive.

Definition 4.3.6. Let $\mathrm{D}$ be a subset of a vector space. Then the configuration induced by $\mathrm{D}$ is $\left(\mathrm{D}, \mathrm{D}, \mathrm{id}_{\mathrm{D}}\right)$.

We shall frequently identify D with the configuration that it induces, saying "the configuration D," etc. This simplifies the statements and proofs of a number of results.

Proposition 4.3.7. Let $\mathcal{D}$ be a configuration having point set $\mathrm{D}$ containing $\mathrm{D}^{\prime}$. Then $\mathrm{D}^{\prime} \leqslant \mathcal{D}$ up to an equivalence of configurations. In particular, $\widetilde{\mathcal{D}} \leqslant \mathcal{D}$ up to an equivalence of configurations.

Proof. Write $\mathcal{D}=(D, E, \ell)$. Let $\chi$ be a choice function for $\left\{\ell^{-1}\left\{d^{\prime}\right\} \mid d^{\prime} \in\right.$ $\left.D^{\prime}\right\}$ and let $E^{\prime}=\operatorname{im}(\chi)$. Then $\left(D^{\prime}, E^{\prime},\left.\ell\right|_{E^{\prime}}\right) \leqslant \mathcal{D}$ and $\left(\mathrm{id}_{\left\langle\mathrm{D}^{\prime}\right\rangle}, \chi\right): \mathrm{D}^{\prime} \rightarrow$ $\left(D^{\prime}, E^{\prime},\left.\ell\right|_{E^{\prime}}\right)$ is an equivalence of configurations. Consequently $D^{\prime} \leqslant \mathcal{D}$ up to an equivalence of configurations. As the point set of $\widetilde{\mathcal{D}}$ is a subset of $D$ by definition, it follows that $\widetilde{\mathcal{D}} \leqslant \mathcal{D}$ up to an equivalence of configurations also.

Some elementary properties of subconfigurations include 
- $\mathcal{D}_{j} \leqslant \bigcup_{i \in I} \mathcal{D}_{i}$ for every $j \in I$ whenever $\left\{\mathcal{D}_{\mathfrak{i}} \mid i \in I\right\}$ is a collection of configurations for which $\bigcup_{i \in I} \mathcal{D}_{i}$ is defined,

- $\bigcap_{i \in I} \mathcal{D}_{i} \leqslant \mathcal{D}_{j}$ for every $j \in I$ whenever $\left\{\mathcal{D}_{i} \mid i \in I\right\}$ is a collection of configurations for which $\bigcap_{i \in \mathrm{I}} \mathcal{D}_{i}$ is defined, and

- $\mathcal{D} \leqslant \bigcap_{i \in I} \mathcal{D}_{i}$ whenever $\{\mathcal{D}\} \sqcup\left\{\mathcal{D}_{i} \mid i \in I\right\}$ is a collection of configurations such that $\mathcal{D} \leqslant \mathcal{D}_{i}$ for every $i \in I$ and $\bigcap_{i \in I} \mathcal{D}_{i}$ is defined.

We close this section by noting that restriction commutes with $M[\cdot]$.

Proposition 4.3.8. Let $\mathcal{D}$ be a configuration and let $X \subseteq E(\mathcal{D})$. Then

$$
(M[\mathcal{D}]) \mid X=M[\mathcal{D} \mid X]
$$

and

$$
(M[\mathcal{D}]) \backslash X=M[\mathcal{D} \backslash X]
$$

Proof. Write $\mathcal{D}=(\mathrm{D}, \mathrm{E}, \ell)$. Clearly is suffices to demonstrate the first equality. We observe that

$$
\begin{aligned}
& Y \in \mathcal{J}((M[\mathcal{D}]) \mid X) \\
\Longleftrightarrow & Y \subseteq X \text { and } Y \in \mathcal{J}(M[\mathcal{D}]) \\
\Longleftrightarrow & Y \subseteq X \text { and }\left.\ell\right|_{Y} \text { is injective and } \ell[Y] \text { is linearly independent } \\
\Longleftrightarrow & Y \subseteq X \text { and }\left.\left(\left.\ell\right|_{X}\right)\right|_{Y} \text { is injective and }\left.\ell\right|_{X}[Y] \text { is linearly independent } \\
\Longleftrightarrow & Y \in \mathcal{J}(M[\mathcal{D} \mid X]) .
\end{aligned}
$$

\subsection{Contraction}

While restrictions are frequently encountered in Chapters 5 and 6, contractions play a more important role in the theory developed. This section gives a definition of contraction for configurations and derives some of its 
properties. Loops are kept rather than deleted in the definition, in contrast with contraction for independence spaces. In this regard, we follow [GGW02].

Definition 4.4.1. Let $\mathcal{D}=(D, E, \ell)$ be a configuration and let $X \subseteq E$. Then the contraction of $\mathcal{D}$ by $X$, denoted $\mathcal{D} / X$, is $(\pi[D], E, \pi \circ \ell)$, where $\pi$ is a projection of $\langle D\rangle$ along $\langle\ell[X]\rangle$ onto a complement of $\langle\ell[X]\rangle$ in $\langle D\rangle$.

The use of $\langle\mathrm{D}\rangle$ in this definition avoids explicit reference to the configuration's ambient vector space.

Whenever $\mathrm{V}$ is a vector space, $\mathrm{W} \leqslant \mathrm{V}$ and $v, v^{\prime} \in \mathrm{V}$, we say that $v$ and $v^{\prime}$ are equivalent modulo $W$, denoted $v \equiv v^{\prime} \bmod W$, if $v=v^{\prime}+w$ for some $w \in W$. It is easily shown that equivalence modulo a subspace is an equivalence relation that respects linear combinations.

Proposition 4.4.2. Every contraction of a $\mathrm{V}$-configuration is a $\mathrm{V}$-configuration that is defined uniquely up to an equivalence of configurations.

Proof. Let $\mathcal{D}=(D, E, \ell)$ be a $V$-configuration and let $X \subseteq E$. If $X$ is empty, the result is trivial, so suppose otherwise. Clearly every contraction of $\mathcal{D}$ by $X$ is a $V$-configuration, so let $\mathcal{D}_{1}=\left(D_{1}, E, \ell_{1}\right)$ and $\mathcal{D}_{2}=\left(D_{2}, E, \ell_{2}\right)$ be two contractions of $\mathcal{D}$ by $X$, where $\mathcal{D}_{i}$ given by $\pi_{i}:\langle D\rangle \rightarrow U_{i}$ for each $i$. Taking $X=\mathrm{id}_{\mathrm{E}}$, we only need to demonstrate the existence of a suitable isomorphism $\phi$.

Let $\left\{\pi_{1}\left(d_{i}\right) \mid i \in I\right\}$ be a basis for $\left\langle\pi_{1}[D]\right\rangle$ contained in $\pi_{1}[D]$. We establish two claims.

(1) $\left\{\pi_{2}\left(\mathrm{~d}_{\mathrm{i}}\right) \mid i \in \mathrm{I}\right\}$ is linearly independent.

For suppose otherwise. Then there exist distinct $\pi_{2}\left(d_{1}\right), \ldots, \pi_{2}\left(d_{n}\right)$ and scalars $\mu_{1}, \ldots, \mu_{n}$ not all zero such that $\sum_{i=1}^{n} \mu_{i} \pi_{2}\left(d_{i}\right)=\mathbf{0}$. Consequently $\sum_{i=1}^{n} \mu_{i} \pi_{2}\left(d_{i}\right) \equiv 0 \bmod \langle\ell[X]\rangle$. As $\pi_{2}\left(d_{i}\right) \equiv d_{i} \equiv \pi_{1}\left(d_{i}\right)$ $\bmod \langle\ell[X]\rangle$ for each $i$, it follows that $\sum_{i=1}^{n} \mu_{i} \pi_{1}\left(d_{i}\right) \equiv 0 \bmod \langle\ell[X]\rangle$ also. So as $\pi_{1}[\mathrm{D}] \subseteq \mathrm{U}_{1}$ and $\mathrm{U}_{1} \cap\langle\ell[\mathrm{X}]\rangle=\{\mathbf{0}\}$, clearly $\sum_{i=1}^{n} \mu_{i} \pi_{2}\left(\mathrm{~d}_{\mathfrak{i}}\right)=\mathbf{0}$, 
contradicting the linear independence of $\left\{\pi_{1}\left(d_{i}\right) \mid i \in I\right\}$. Consequently $\left\{\pi_{2}\left(d_{i}\right) \mid i \in I\right\}$ is linearly independent.

(2) $\left\{\pi_{2}\left(d_{i}\right) \mid i \in I\right\}$ is a basis for $\left\langle\pi_{2}[D]\right\rangle$.

Let $d \in D$. As $\left\{\pi_{1}\left(d_{i}\right) \mid i \in I\right\}$ is a basis for $\left\langle\pi_{1}[D]\right\rangle$, there exist $\pi_{1}\left(d_{1}\right)$, $\ldots, \pi_{1}\left(d_{n}\right)$ and scalars $\mu_{1}, \ldots, \mu_{n}$ such that $\pi_{1}(d)=\sum_{i=1}^{n} \mu_{i} \pi_{1}\left(d_{i}\right)$. Repeating the argument of (1) shows that $\pi_{2}(d)=\sum_{i=1}^{n} \mu_{i} \pi_{2}\left(d_{i}\right)$. Hence $\left\{\pi_{2}\left(d_{i}\right) \mid i \in I\right\}$ is a basis for $\left\langle\pi_{2}[D]\right\rangle$.

It follows from (2) that $\phi: \pi_{1}\left(d_{i}\right) \mapsto \pi_{2}\left(d_{i}\right)$ is a bijection from a basis of $\left\langle\pi_{1}[\mathrm{D}]\right\rangle$ to a basis of $\left\langle\pi_{2}[\mathrm{D}]\right\rangle$. Extend $\phi$ linearly to an isomorphism $\left\langle\pi_{1}[\mathrm{D}]\right\rangle \rightarrow\left\langle\pi_{2}[\mathrm{D}]\right\rangle$ and let $e \in E$. Then there exist $\pi_{1}\left(\mathrm{~d}_{1}\right), \ldots, \pi_{1}\left(\mathrm{~d}_{n}\right)$ and scalars $\mu_{1}, \ldots, \mu_{n}$ such that

$$
\begin{aligned}
\ell_{1}(e)=\pi_{1}(\ell(e)) & =\sum_{i=1}^{n} \mu_{i} \pi_{1}\left(d_{i}\right) \\
\phi\left(\ell_{1}(e)\right) & =\phi\left(\sum_{i=1}^{n} \mu_{i} \pi_{1}\left(d_{i}\right)\right) \\
& =\sum_{i=1}^{n} \mu_{i} \phi\left(\pi_{1}\left(d_{i}\right)\right) \\
& =\sum_{i=1}^{n} \mu_{i} \pi_{2}\left(d_{i}\right) .
\end{aligned}
$$

Thus $\phi\left(\ell_{1}(e)\right) \equiv \ell_{1}(e) \bmod \langle\ell[X]\rangle$. As $\ell_{1}(e) \equiv \ell_{2}(e) \bmod \langle\ell[X]\rangle$ also, it follows that $\phi\left(\ell_{1}(e)\right) \equiv \ell_{2}(e) \bmod \langle\ell[X]\rangle$. So as $\phi\left(\ell_{1}(e)\right), \ell_{2}(e) \in \mathrm{U}_{2}$ and $\mathrm{U}_{2} \cap\langle\ell[X]\rangle=\{\boldsymbol{0}\}$, necessarily $\phi\left(\ell_{1}(e)\right)=\ell_{2}(e)$. Hence $(\phi, \chi): \mathcal{D}_{1} \rightarrow \mathcal{D}_{2}$ is an equivalence of configurations.

The following proposition is an obvious consequence of the definition of contraction. 
Proposition 4.4.3. Let $\mathcal{D}$ be a configuration and let $X \subseteq E(\mathcal{D})$. Then $\mathcal{D} / X=$ $\mathcal{D} / \mathrm{B}_{\mathrm{X}}$ up to an equivalence of configurations for every $\mathrm{B}_{\mathrm{X}} \in \mathcal{B}(\mathcal{D} \mid \mathrm{X})$.

The next proposition is the analogue of Proposition 3.6.3 for configurations.

Proposition 4.4.4. Let $\mathcal{D}$ be a configuration and let $\mathrm{X}, \mathrm{Y} \subseteq \mathrm{E}(\mathcal{D})$ be disjoint. Then

(1) $(\mathcal{D} \backslash X) \backslash Y=\mathcal{D} \backslash(X \sqcup Y)=(\mathcal{D} \backslash Y) \backslash X$,

(2) $(\mathcal{D} / \mathrm{X}) / \mathrm{Y}=\mathcal{D} /(\mathrm{X} \sqcup \mathrm{Y})=(\mathcal{D} / \mathrm{Y}) / \mathrm{X}$ up to an equivalence of configurations, and

(3) $(\mathcal{D} \backslash X) / Y=(\mathcal{D} / Y) \backslash X$ up to an equivalence of configurations.

Proof. Write $\mathcal{D}=(\mathrm{D}, \mathrm{E}, \ell)$. The statement (1) clearly holds, so we demonstrate (2) and (3).

In order to establish (2), it suffices by symmetry to demonstrate that $(\mathcal{D} / \mathrm{X}) / \mathrm{Y}=\mathcal{D} /(\mathrm{X} \sqcup \mathrm{Y})$ up to an equivalence of configurations. Clearly each has ground set $E$. Let $\pi$ be a projection of $\langle D\rangle$ along $W:=\langle\ell[X]\rangle$ onto some complement $\mathrm{U}$ of $\mathrm{W}$ in $\langle\mathrm{D}\rangle$.

Clearly $W^{\prime}:=\langle\pi[\ell[\mathrm{Y}]]\rangle \leqslant \mathrm{U}$. Let $\pi^{\prime}$ be a projection of $\mathrm{U}$ along $\mathrm{W}^{\prime}$ onto some complement $\mathrm{U}^{\prime}$ of $\mathrm{W}^{\prime}$ in $\mathrm{U}$. Then $\pi$ determines a contraction $\mathcal{D}^{\prime}$ of $\mathcal{D}$ by $\mathrm{X}$, and $\pi^{\prime}$ determines a contraction of $\mathcal{D}^{\prime}$ by $\mathrm{Y}$.

Moreover, $\operatorname{ker}\left(\pi^{\prime} \circ \pi\right)=\mathrm{W} \oplus \mathrm{W}^{\prime}=\langle\ell[\mathrm{X} \sqcup \mathrm{Y}]\rangle$ and $\operatorname{im}\left(\pi^{\prime} \circ \pi\right)=\mathrm{U}^{\prime}$. Consequently $\pi^{\prime} \circ \pi$ determines a contraction of $\mathcal{D}$ by $X \sqcup \mathrm{Y}$, and so (2) holds.

We now consider (3). Clearly $(\mathcal{D} \backslash X) / Y$ and $(\mathcal{D} / Y) \backslash X$ each have ground set $E-X$. Let $\pi$ be a projection of $\langle D\rangle$ along $W:=\langle\ell[Y]\rangle$ onto some complement $\mathrm{U}$ of $\mathrm{W}$ in $\langle\mathrm{D}\rangle$. Then $\pi$ determines a contraction of $\mathcal{D}$ by $\mathrm{Y}$.

Moreover, it follows from the Modular Law that $\pi^{\prime}:=\left.\pi\right|_{\langle\ell[E-X]\rangle}$ is the projection of $\mathrm{V}:=\langle\ell[E-X]\rangle$ along $W$ onto $U \cap V$. So $\pi^{\prime}$ determines a contraction of $\mathcal{D} \backslash X$ by $Y$. Finally, we observe that $\left.(\pi \circ \ell)\right|_{E-X}=\pi^{\prime} \circ\left(\left.\ell\right|_{E-X}\right)$. Hence (3) holds. 
As contraction in configurations does not involve the deletion of loops, contraction "almost commutes" with $M[\cdot]$.

Proposition 4.4.5. Let $\mathcal{D}$ be a configuration and let $\mathrm{X} \subseteq \mathrm{E}(\mathcal{D})$. Then

$$
(M[\mathcal{D} / X]) \backslash X=(M[\mathcal{D}]) / X
$$

Proof. Write $\mathcal{D}=(\mathrm{D}, \mathrm{E}, \ell)$, let $\pi$ determine a contraction of $\mathcal{D}$ by $X$ and let $\mathrm{B}_{\mathrm{X}} \in \mathcal{B}(\mathcal{D} \mid \mathrm{X})$. Then

$$
\begin{aligned}
& Y \in \mathcal{J}((M[\mathcal{D}]) / X) \\
\Longleftrightarrow & Y \sqcup B_{X} \in \mathcal{J}(M[\mathcal{D}]) \\
\Longleftrightarrow & \left.\ell\right|_{Y} \sqcup B_{X} \text { is injective and } \ell\left[Y \sqcup B_{X}\right] \text { is linearly independent } \\
\Longleftrightarrow & \left.\ell\right|_{Y} \text { and }\left.\ell\right|_{B_{X}} \text { are injective, and } \ell[Y] \sqcup \ell\left[B_{X}\right] \text { is linearly independent } \\
\Longleftrightarrow & \left.(\pi \circ \ell)\right|_{Y} \text { is injective and }(\pi \circ \ell)[Y] \text { is linearly independent } \\
\Longleftrightarrow & Y \in \mathcal{J}((M[\mathcal{D} / X]) \backslash X) .
\end{aligned}
$$

The next proposition is a restatement of Claim 5.3 of [HW06]. It is used in Section 6.2 for establishing the finite case of the main theorem.

Proposition 4.4.6. Let $\mathcal{D}$ be a finite-dimensional configuration having point set $\mathrm{D}=\bigsqcup_{i=0}^{\mathrm{n}} \mathrm{D}_{\mathrm{i}}$ and let

$$
S=\left\langle D_{0}\right\rangle+\sum_{i=1}^{n}\left(\left\langle D_{i}\right\rangle \cap\left\langle D-D_{i}\right\rangle\right)
$$

Then

$$
\operatorname{dim}(S)=\sum_{i=1}^{n} \operatorname{dim}\left(\left\langle D-D_{i}\right\rangle\right)-(n-1) \operatorname{dim}(\mathcal{D})
$$

Our argument is a restatement of that given on p. 1125 of [HW06]. 
Proof. We demonstrate by induction that

$$
\operatorname{dim}(S)=\sum_{i=1}^{n} r_{M}\left(D-D_{i}\right)-(n-1) r(M)
$$

where $M:=M[\widetilde{\mathcal{D}}]$. The result then follows from the correspondence between rank in independence spaces and dimension in configurations.

When $n=1, S=\left\langle D_{0}\right\rangle=\left\langle D-D_{1}\right\rangle$, so $\operatorname{dim}(S)=\operatorname{dim}\left(\left\langle D-D_{1}\right\rangle\right)=$ $r_{M}\left(D-D_{1}\right)$, and (4.4.1) holds. Now suppose that $n>1$ and that (4.4.1) holds for $n-1$. Then

$$
\begin{aligned}
\operatorname{dim}(S)= & \operatorname{dim}\left(S /\left\langle D_{n}\right\rangle\right)+\operatorname{dim}\left(S \cap\left\langle D_{n}\right\rangle\right) \\
= & \sum_{i=1}^{n-1} r_{M\left[\widetilde{\mathcal{D}} / D_{n}\right]}\left(\left(D-D_{n}\right)-D_{i}\right)-(n-2) r\left(M\left[\widetilde{\mathcal{D}} / D_{n}\right]\right) \\
& +\operatorname{dim}\left(\left\langle D-D_{n}\right\rangle \cap\left\langle D_{n}\right\rangle\right) \\
= & \sum_{i=1}^{n-1} r_{M / D_{n}}\left(\left(D-D_{n}\right)-D_{i}\right)-(n-2) r\left(M / D_{n}\right) \\
& +\operatorname{dim}\left(\left\langle D-D_{n}\right\rangle\right)+\operatorname{dim}\left(\left\langle D_{n}\right\rangle\right)-\operatorname{dim}(\langle D\rangle) \\
& \sum_{i=1}^{n-1} r_{M}\left(D-D_{i}\right)-(n-1) r_{M}\left(D_{n}\right)-(n-2) r(M) \\
& +(n-2) r_{M}\left(D_{n}\right)+r_{M}\left(D-D_{n}\right)+r_{M}\left(D_{n}\right)-r(M) \\
= & \sum_{i=1}^{n} r_{M}\left(D-D_{i}\right)-(n-1) r(M) .4 .5
\end{aligned}
$$

Hence (4.4.1) holds.

The following technical proposition allows the "local" replacement of a contraction by a "finite" contraction. This result is applied in Sec- 
tions 5.3 and 6.4 and Cahpter 7.

Proposition 4.4.7. Let $\mathcal{D}=(\mathrm{D}, \mathrm{E}, \ell)$ be a configuration, let $\pi$ determine a contraction of $\mathcal{D}$ by $\mathrm{X} \subseteq \mathrm{E}$ and let $\mathrm{Y} \subseteq \pi[\mathrm{D}]$ be finite. Then there exist finite $\mathrm{X}_{0} \subseteq \mathrm{X}$ and $\pi^{\prime}$ determining a contraction of $\mathcal{D}$ by $\mathrm{X}_{0}$ such that $\mathrm{Y} \subseteq \pi^{\prime}[\mathrm{D}]$ and $\pi=\pi^{\prime \prime} \circ \pi^{\prime}$ for some projection $\pi^{\prime \prime}$ fixing $\operatorname{im}(\pi)$.

Proof. Write $Y=\left\{y_{1}, \ldots, y_{n}\right\}$. Then for each $i$, there exist $d_{i} \in D$ and finite $X_{i} \subseteq X$ such that $y_{i} \equiv d_{i} \bmod \left\langle\ell\left[X_{i}\right]\right\rangle$. Let $X_{0}=\bigcup_{i=1}^{n} X_{i}$ and let $\pi^{\prime}$ be the projection of $\langle\mathrm{D}\rangle$ along $W:=\left\langle\ell\left[X_{0}\right]\right\rangle$ onto $\operatorname{im}(\pi) \oplus \mathrm{U}$, where $\langle\ell[X]\rangle=\mathrm{U} \oplus \mathrm{W}$. Then $\pi^{\prime}$ determines a contraction of $\mathcal{D}$ by $\mathrm{X}_{0}$ such that $Y \subseteq \pi^{\prime}[D]$. Moreover, $\pi=\pi^{\prime \prime} \circ \pi^{\prime}$, where $\pi^{\prime \prime}$ is the projection of $\langle\mathrm{D}\rangle$ along U onto $\operatorname{im}(\pi) \oplus W$.

\subsection{Connectivity}

This section examines connectivity in configurations. Like many concepts for configurations, it corresponds closely to its analogue for general independence spaces. However, it may be defined without taking a difference of dimensions (ranks in general), making it applicable even to infinite-dimensional configurations. This is because a suitable subspace can always be induced, even if the configuration itself has a paucity of points in the region whose dimension is to be determined (see Figure 4.3).

Definition 4.5.1. Let $\mathcal{D}=(D, E, \ell)$ be a configuration. Define $\lambda_{\mathcal{D}}:\{(X, Y) \mid$ $\mathrm{X} \cup \mathrm{Y}=\mathrm{E}\} \rightarrow \omega \sqcup\{\infty\}$ by

$$
\lambda_{\mathcal{D}}(X, Y)=\operatorname{dim}(\langle\ell[X]\rangle \cap\langle\ell[Y]\rangle)
$$

whenever $X, Y \subseteq E$ are such that $X \cup Y=E$, and define $\lambda_{\mathcal{D}}: \mathcal{P}(E) \rightarrow$ $\omega \sqcup\{\infty\}$ by

$$
\lambda_{\mathcal{D}}(X)=\lambda_{\mathcal{D}}(X, E-X)
$$




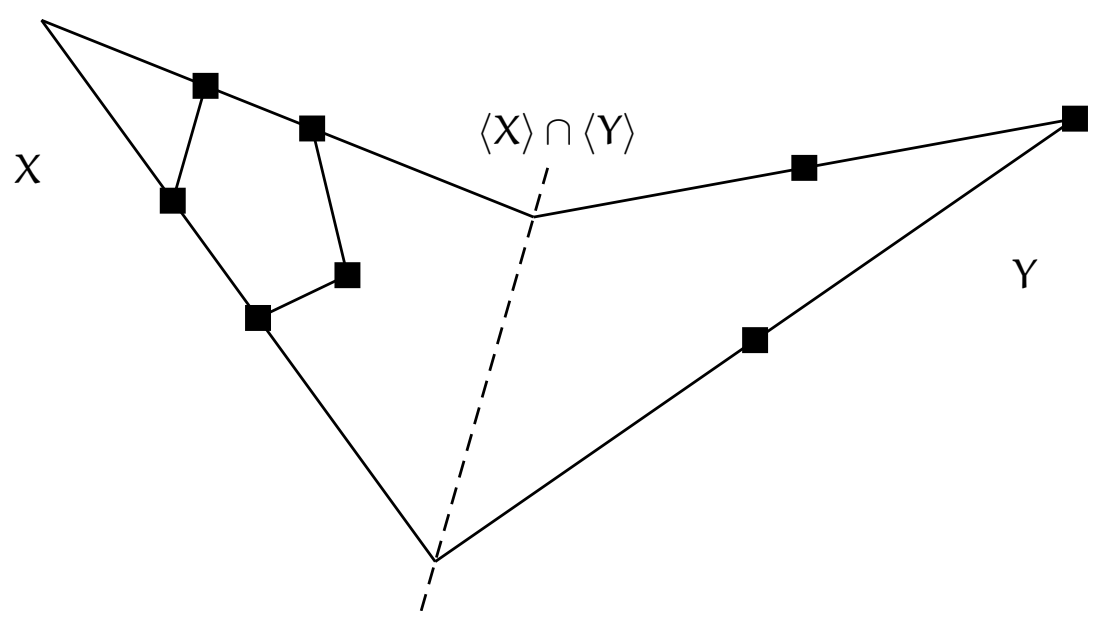

Figure 4.3: A geometric representation of a configuration $D$ exhibiting a "split" into two flats $X$ and $Y$. Even though $\langle X\rangle \cap\langle Y\rangle \cap D$ is empty, $\langle X\rangle \cap\langle Y\rangle$ still exists as a set of points in the ambient vector space.

for every $X \subseteq E$. Then define $\kappa_{\mathcal{D}}: \mathcal{P}(E) \times \mathcal{P}(E) \rightarrow \omega \sqcup\{\infty\}$ by

$$
\kappa_{\mathcal{D}}\left(X^{\prime}, Y^{\prime}\right)=\inf \left\{\lambda_{\mathcal{D}}(X, Y) \mid X^{\prime} \subseteq X, Y^{\prime} \subseteq Y, X \cup Y=E\right\}
$$

for every $X^{\prime}, Y^{\prime} \subseteq E$. The subscript " $\mathcal{D}$ " is omitted when there is no ambiguity.

The following proposition lists some natural observations concerning these connectivity functions.

Proposition 4.5.2. Let $\mathcal{D}=(\mathrm{D}, \mathrm{E}, \ell)$ be a configuration. Then

(1) $\lambda_{\mathcal{D}}(X, Y)=\lambda_{D}(\ell[X], \ell[Y])=\lambda_{\widetilde{D}}(X \cap E(\widetilde{D}), Y \cap E(\widetilde{D}))$ whenever $X, Y \subseteq E$ are such that $\mathrm{X} \cup \mathrm{Y}=\mathrm{E}$,

(2) $\lambda_{\mathcal{D}}(X)=\lambda_{D}(\ell[X])=\lambda_{\widetilde{\mathcal{D}}}(X \cap E(\widetilde{D}))$ for every $X \subseteq E$, and

(3) $\kappa_{\mathcal{D}}(X, Y)=\kappa_{D}(\ell[X], \ell[Y])=\kappa_{\widetilde{\mathcal{D}}}(X \cap E(\widetilde{D}), Y \cap E(\widetilde{D}))$ for every $X, Y \subseteq E$. 
So it suffices in the context of connectivity to simply view configurations as subsets of their ambient vector spaces.

The next proposition is an analogue of Proposition 3.9.2 for configurations.

Proposition 4.5.3. For every configuration,

(1) $\lambda(\cdot, \cdot), \lambda(\cdot)$ and $\mathrm{\kappa}(\cdot, \cdot)$ are each symmetric, and

(2) $\mathrm{\kappa}(\cdot, \cdot)$ is increasing in each of its arguments.

The following proposition says that a subset of a configuration may be replaced by its closure without affecting either $\lambda$ or $\kappa$.

Proposition 4.5.4. Let $\mathrm{D}$ be a configuration. Then

(1) $\lambda(X, Y)=\lambda(D \cap\langle X\rangle, Y)$ whenever $X, Y \subseteq D$ are such that $X \cup Y=D$,

(2) $\lambda(X)=\lambda(D \cap\langle X\rangle)$ for every $X \subseteq D$, and

(3) $\kappa(X, Y)=\kappa(D \cap\langle X\rangle, Y)$ for every $X, Y \subseteq D$.

We now confirm that $\lambda$ and $\kappa$ for configurations coincide with their analogues for general independence spaces in the finite-dimensional case (that is, when the latter are defined).

Proposition 4.5.5. Let $\mathcal{D}$ be a finite-dimensional configuration. Then $\lambda_{\mathcal{D}}=$ $\lambda_{M[\mathcal{D}]}$ and $\mathrm{K}_{\mathcal{D}}=\mathrm{\kappa}_{\mathrm{M}[\mathcal{D}]}$.

Proof. Write $\mathcal{D}=(\mathrm{D}, \mathrm{E}, \ell)$ and let $\mathrm{X}, \mathrm{Y} \subseteq \mathrm{E}$ be such that $\mathrm{X} \cup \mathrm{Y}=\mathrm{E}$. Then

$$
\begin{aligned}
\lambda_{\mathcal{D}}(X, Y) & =\operatorname{dim}(\langle\ell[X]\rangle \cap\langle\ell[Y]\rangle) \\
& =\operatorname{dim}(\langle\ell[X]\rangle)+\operatorname{dim}(\langle\ell[Y]\rangle)-\operatorname{dim}(\mathcal{D}) \\
& =r_{M[\mathcal{D}]}(X)+r_{M[\mathcal{D}]}(Y)-r(M[\mathcal{D}]) \\
& =\lambda_{M[\mathcal{D}]}(X, Y) .
\end{aligned}
$$

Hence $\lambda_{\mathcal{D}}=\lambda_{M[\mathcal{D}]}$. The result for $\kappa$ is then clear. 
Submodularity then follows from Proposition 3.9.4.

Corollary 4.5.6. $\lambda(\cdot)$ is submodular for every finite-dimensional configuration. 


\section{Chapter 5}

\section{Further Notions}

This chapter encompasses a trio of lesser-known topics. Each arose as an appropriate tool for the solution of a particular problem for tree-decompositions of configurations (see Chapter 6). While this was the original motivation, the ideas involved also have their own intrinsic merit. Consequently some further development has been given.

\subsection{Bridges}

The aim of this section is to develop an analogue of a concept from graph theory. Given a graph $G$ and a subgraph $X$ of $G$, the bridges of $X$ in $G$ (or the components mod $X$ of $G$ ) are the connected components of $G-X$ (see Figure 5.1). Some theory of bridges in graphs may be found on pp. 27-30 of [Tut01]. Note that some authors use "bridge" to refer to an isthmus, an edge that separates its endvertices.

The-perhaps initially surprising-difference in the matroidal setting is the replacement of deletion by contraction. This can be understood by considering graph $G$ and its cycle independence space $M(G)$. Deleting $X$ from $G$ is equivalent to

(1) contracting $E_{G}[X]$ in $G$, and then 
(2) deleting $V\left[X / E_{G}[X]\right]$ from $G / E_{G}[X]$.

As connectivity in $\mathrm{M}(\mathrm{G})$ corresponds to 2-connectivity in $\mathrm{G}$ (see p. 127 of [Ox104]), step (2) is unimportant from a matroidal perspective. So deleting $X$ from $G$ effectively corresponds to contracting $E_{G}[X]$ in $M(G)$.

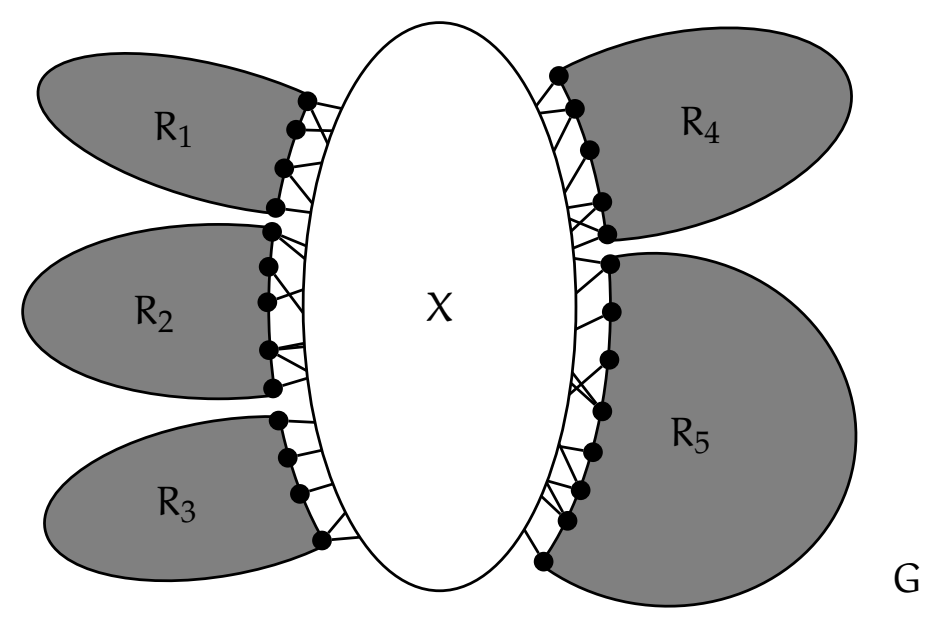

Figure 5.1: Bridges of $X$ in a graph $G$.

Definition 5.1.1. Let $M$ be an independence space on $E$. Define $\gamma_{M}: E \rightarrow$ $\mathcal{P}(\mathrm{E})$ by

$$
\gamma_{M}(e)=\{e\} \cup\{f \in E \mid(\exists C \in \mathcal{C}(M))\{e, f\} \subseteq C\}
$$

for every $e \in E$. Then $\gamma_{M}(\cdot)$ induces a relation $\gamma_{M}$ on $E$, given by $e \gamma_{M} f$ if and only if $e \in \gamma_{M}(f)$. As usual, the subscript " $M$ " is omitted when there is no ambiguity.

Proposition 5.1.2. Let $M$ be an independence space. Then $\gamma_{M}$ is an equivalence relation on $\mathrm{E}(\mathrm{M})$.

For completeness, we reproduce the argument given for matroids on pp. 124-125 of [Ox104]. 
Proof. Clearly $\gamma$ is reflexive and symmetric. So we demonstrate transitivity: suppose that $e \in \gamma(f)$ and $f \in \gamma(g)$, where $e, f, g \in E(M)$ are distinct. Then $g \in \gamma(f)$ by symmetry, so there exist $C_{1}, C_{2} \in \mathcal{C}(M)$ such that $e \in C_{1}$, $g \in C_{2}$ and $C_{1} \cap C_{2}$ is non-empty. Without loss of generality, $\left|C_{1} \cup C_{2}\right|$ is minimal. We wish to show that $M$ has a circuit containing $\{e, g\}$. Aiming for a contradiction, suppose otherwise.

Then $\mathrm{C}_{1} \neq \mathrm{C}_{2}$. Let $\mathrm{h} \in \mathrm{C}_{1} \cap \mathrm{C}_{2}$. By Strong Circuit Elimination, there exists $C_{3} \in \mathcal{C}(M)$ such that $e \in C_{3} \subseteq\left(C_{1} \cup C_{2}\right)-\{h\}$. By hypothesis, $g \notin C_{3}$. As $C_{3} \nsubseteq C_{1}$, there exists $i \in\left(C_{2}-C_{1}\right) \cap C_{3}$. Applying Strong Circuit Elimination again, we see that there exists $C_{4} \in \mathcal{C}(M)$ such that $\mathrm{g} \in \mathrm{C}_{4} \subseteq\left(\mathrm{C}_{2} \cup \mathrm{C}_{3}\right)-\{i\}$. As $\mathrm{C}_{4} \nsubseteq \mathrm{C}_{2}$, necessarily $\mathrm{C}_{4} \cap\left(\mathrm{C}_{3}-\mathrm{C}_{2}\right) \neq \varnothing$, so $\mathrm{C}_{1} \cap \mathrm{C}_{4} \neq \varnothing$. But $\mathrm{C}_{1} \cup \mathrm{C}_{4} \subseteq\left(\mathrm{C}_{1} \cup \mathrm{C}_{2}\right)-\{i\}$, so $\left|\mathrm{C}_{1} \cup \mathrm{C}_{4}\right|<\left|\mathrm{C}_{1} \cup \mathrm{C}_{2}\right|$, contradicting the choice of $\left(C_{1}, C_{2}\right)$. Hence $M$ has a circuit containing $\{e, g\}$, and so $\gamma$ is transitive.

Definition 5.1.3. Let $M$ be an independence space and let $X \subseteq E(M)$. Then an $\mathrm{X}$-bridge of $\mathrm{M}$ is a $\gamma_{M / X}$-equivalence class. A connected component of $M$ is a $\varnothing$-bridge of $M$. A separator of $M$ is a union of connected components of $M$. The empty separator of $M$ is $\varnothing$.

Let $\mathcal{D}$ be a configuration and let $X \subseteq \mathrm{E}(\mathcal{D})$. Then an $X$-bridge of $\mathcal{D}$ is an $X$-bridge of $M[\mathcal{D}]$. The notions of connected component, separator and empty separator are then inherited from independence spaces.

It follows immediately from the definition that the connected components of $M$ are precisely its $\gamma$-equivalence classes.

We now give some useful characterisations of unions of $X$-bridges, which are separators when $X$ is empty. We first consider "combining bases."

Proposition 5.1.4. Let $M$ be an independence space on $E$, let $X \subseteq E$, let $\Re \subseteq$ $\mathrm{E}-\mathrm{X}$ and let $\overline{\mathfrak{R}}=(\mathrm{E}-\mathrm{X})-\mathfrak{R}$. Then $\mathfrak{R}$ is a union of $\mathrm{X}$-bridges of $\mathrm{M}$ if and only if $\mathrm{B}_{1} \sqcup \mathrm{B}_{\mathrm{X}} \sqcup \mathrm{B}_{2} \in \mathcal{B}(\mathrm{M})$ whenever $\mathrm{B}_{\mathrm{X}} \in \mathcal{B}(\mathrm{M} \mid \mathrm{X})$ is extended by $\mathrm{B}_{1}$ to a basis of $\mathrm{M} \mid(\Re \sqcup \mathrm{X})$ and (independently) extended by $\mathrm{B}_{2}$ to a basis of $\mathrm{M} \mid(\mathrm{X} \sqcup \overline{\mathfrak{R}})$. 
Proof. Suppose that $\Re$ is a union of $X$-bridges of $M$ and let $B_{X} \in \mathcal{B}(M \mid X)$. Extend $B_{X}$ by $B_{1}$ to a basis of $M \mid(\Re \sqcup X)$ and (independently) by $B_{2}$ to a basis of $M \mid(X \sqcup \bar{\Re})$. Let $B=B_{1} \sqcup B_{X} \sqcup B_{2}$ and suppose that $B \notin \mathcal{J}(M)$. Then $B$ contains some $C \in \mathcal{C}(M)$. In light of $(C 4)$, we may assume that $|C-X|$ is minimal without loss of generality. As $\Re$ is a union of $X$-bridges, $C$ cannot properly meet both $\mathfrak{R}$ and $\bar{\Re}$. So either $C \subseteq \mathrm{B}_{1} \sqcup \mathrm{B}_{\mathrm{X}}$ or $\mathrm{C} \subseteq \mathrm{B}_{\mathrm{X}} \sqcup \mathrm{B}_{2}$, which is impossible. Thus $B \in \mathcal{J}(M)$. As $B_{1} \sqcup B_{X}$ and $B_{X} \sqcup B_{2}$ are maximal $\mathcal{J}(M)$-subsets of $\Re \sqcup X$ and $X \sqcup \bar{\Re}$ respectively, $B$ is a maximal $\mathcal{J}(M)$-subset of $E$, that is, $B \in \mathcal{B}(M)$.

Now suppose that $B_{1} \sqcup B_{X} \sqcup B_{2} \in \mathcal{B}(M)$ whenever $B_{X} \in \mathcal{B}(M \mid X)$ is extended by $B_{1}$ to a basis of $M \mid(\Re \sqcup X)$ and (independently) extended by $B_{2}$ to a basis of $M \mid(X \sqcup \bar{\Re})$, and that $\Re$ is not a union of X-bridges of $M$. Then there exist $e \in \Re$ and $f \in \bar{\Re}$ such that $f \in \gamma_{M / X}(e)$. But then there exists $C \in \mathcal{C}(M)$ containing $\{e, f\}$. Each of $C \cap \Re$ and $C \cap \bar{\Re}$ is independent, so there exist (independent) extensions $B_{1} \supseteq C \cap \Re$ and $\mathrm{B}_{2} \supseteq \mathrm{C} \cap \bar{\Re}$ of some $\mathrm{B}_{\mathrm{X}} \in \mathcal{B}(\mathrm{M} \mid \mathrm{X})$ containing $\mathrm{C} \cap \mathrm{X}$ to bases of $\mathrm{M} \mid(\Re \sqcup \mathrm{X})$ and $M \mid(X \sqcup \bar{\Re})$ respectively. It follows that $C \subseteq B_{1} \sqcup B_{X} \sqcup B_{2}$, contradicting the independence of $B_{1} \sqcup B_{X} \sqcup B_{2}$. Hence $\mathfrak{R}$ is a union of X-bridges of M.

Corollary 5.1.5. Let $M$ be an independence space on $E$ and let $\mathfrak{K} \subseteq E$. Then $\mathfrak{K}$ is a separator of $M$ if and only if $\mathrm{B}_{\mathfrak{K}} \sqcup \mathrm{B}_{\mathrm{E}-\mathfrak{K}} \in \mathcal{B}(M)$ whenever $\mathrm{B}_{\mathfrak{K}} \in \mathcal{B}(M \mid \mathfrak{K})$ and $\mathrm{B}_{\mathrm{E}-\mathfrak{K}} \in \mathcal{B}(\mathrm{M} \mid(\mathrm{E}-\mathfrak{K}))$.

In the finite-rank case we have the following rank characterisation.

Proposition 5.1.6. Let $M$ be a finite-rank independence space on $E$, let $X \subseteq E$ and let $\mathfrak{R} \subseteq \mathrm{E}-\mathrm{X}$. Then $\mathfrak{R}$ is a union of X-bridges of $\mathrm{M}$ if and only if

$$
r(\Re \sqcup X)-r(X)+r(E-\Re)=r(M) .
$$

Proof. Suppose that $\mathfrak{R}$ is a union of $X$-bridges of $M$. Let $B_{X} \in \mathcal{B}(M \mid X)$. Extend $B_{X}$ by $B_{1}$ to a basis of $M \mid(\Re \sqcup X)$ and (independently) by $B_{2}$ to 
a basis of $M \mid(E-\Re)$. Then $B_{1} \sqcup B_{X} \sqcup B_{2} \in \mathcal{B}(M)$ by Proposition 5.1.4. Consequently

$$
\begin{aligned}
\left|B_{1} \sqcup B_{X}\right|-\left|B_{X}\right|+\left|B_{X} \sqcup B_{2}\right| & =r(M) \\
r(\Re \sqcup X)-r(X)+r(E-\Re) & =r(M) .
\end{aligned}
$$

Now suppose that $r(\Re \sqcup X)-r(X)+r(E-\Re)=r(M)$. Let $B_{X} \in \mathcal{B}(M \mid X)$. Extend $B_{X}$ by $B_{1}$ to a basis of $M \mid(\Re \sqcup X)$ and (independently) by $B_{2}$ to a basis of $M \mid(E-\mathfrak{R})$. Let $B=B_{1} \sqcup B_{X} \sqcup B_{2}$. Then

$$
\begin{aligned}
\left|\mathrm{B}_{1} \sqcup \mathrm{B}_{X}\right|-\left|\mathrm{B}_{X}\right|+\left|\mathrm{B}_{X} \sqcup \mathrm{B}_{2}\right| & =\mathrm{r}(\mathrm{M}) \\
|\mathrm{B}| & =\mathrm{r}(\mathrm{M}) .
\end{aligned}
$$

Moreover, $\Re \sqcup \mathrm{X} \subseteq \mathrm{cl}\left(\mathrm{B}_{1} \sqcup \mathrm{B}_{\mathrm{X}}\right) \subseteq \mathrm{cl}(\mathrm{B})$ and $(\mathrm{E}-\mathfrak{R})-\mathrm{X} \subseteq \mathrm{cl}\left(\mathrm{B}_{\mathrm{X}} \sqcup \mathrm{B}_{2}\right) \subseteq$ $\mathrm{cl}(\mathrm{B})$, so $B$ spans $E$. Hence $B \in \mathcal{B}(M)$, and so $\mathfrak{R}$ is a union of $X$-bridges of $M$ by Proposition 5.1.4.

Corollary 5.1.7. Let $M$ be a finite-rank independence space on $E$ and let $\mathfrak{K} \subseteq \mathrm{E}$. Then $\mathfrak{K}$ is a separator of $M$ if and only if

$$
r(\mathfrak{K})+r(E-\mathfrak{K})=r(M) .
$$

Similar to Corollary 5.1.5, "partitioning a basis yields bases."

Proposition 5.1.8. Let $M$ be an independence space on $E$ and let $\mathfrak{K} \subseteq \mathrm{E}$. Then $\mathfrak{K}$ is a separator of $M$ if and only if $B \cap \mathfrak{K} \in \mathcal{B}(M \mid \mathfrak{K})$ and $B-\mathfrak{K} \in \mathcal{B}(M \mid(E-\mathfrak{K}))$ for every $\mathrm{B} \in \mathcal{B}(M)$.

Proof. Recall that whenever $B \in \mathcal{B}(M)$ and $e \in E(M)-B$, the unique circuit of $M$ containing $e$ and contained in $B \sqcup\{e\}$ is denoted by $C(e, B)$ (see Proposition 3.2.3).

Suppose that $\mathfrak{K}$ is a separator of $M$. Let $B \in \mathcal{B}(M)$. Then $B \cap \mathfrak{K} \subseteq \mathfrak{K}$ and $B-\mathfrak{K} \subseteq E-\mathfrak{K}$ are each independent. Suppose that $B \cap \mathfrak{K} \notin \mathcal{B}(M \mid \mathfrak{K})$. Then there exists $e \in \mathfrak{K}-B$ such that $(B \cap \mathfrak{K}) \sqcup\{e\} \in \mathcal{J}(M)$. Consequently 
$\mathrm{C}(e, \mathrm{~B}) \nsubseteq \mathfrak{K}$, so $\mathrm{C}(e, \mathrm{~B})-\mathfrak{K}$ is non-empty, contradicting $\mathfrak{K}$ being a separator. Thus $B \cap \mathfrak{K} \in \mathcal{B}(M \mid \mathfrak{K})$. By symmetry, $B-\mathfrak{K} \in \mathcal{B}(M \mid(E-\mathfrak{K}))$.

Now suppose that $B \cap \mathfrak{K} \in \mathcal{B}(M \mid \mathfrak{K})$ and $B-\mathfrak{K} \in \mathcal{B}(M \mid(E-\mathfrak{K}))$ whenever $B \in \mathcal{B}(M)$ and that $\mathfrak{K}$ is not a separator of $M$. Then there exists $C \in \mathcal{C}(M)$ such that $C \cap \mathfrak{K}$ and $C-\mathfrak{K}$ are each non-empty. Let $e \in C \cap \mathfrak{K}$ and extend $C-\{e\}$ to a basis $B$ of $M$. Then $B \cap \mathfrak{K} \in \mathcal{B}(M \mid \mathfrak{K})$, so $C(e, B \cap \mathfrak{K})$ is the unique circuit containing $e$ that is contained in $(B \cap \mathfrak{K}) \sqcup\{e\}$. But $\mathrm{C}$ is the unique circuit containing $e$ that is contained in $\mathrm{B} \sqcup\{\boldsymbol{e}\}$, so $C=C(e, B \cap \mathfrak{K}) \subseteq \mathfrak{K}$, contradicting $C-\mathfrak{K} \neq \varnothing$. Hence $\mathfrak{K}$ is a separator of M.

In the finite-rank case, this result could easily have been derived from Corollary 5.1.7. Combining the preceding results gives the following characterisations of $\mathrm{X}$-bridges.

Corollary 5.1.9. Let $\mathrm{M}$ be an independence space on $\mathrm{E}$, let $\mathrm{X} \subseteq \mathrm{E}$ and let $\mathrm{R} \subseteq \mathrm{E}-\mathrm{X}$. Then the following are equivalent:

(1) $\mathrm{R}$ is an $\mathrm{X}$-bridge of $\mathrm{M}$.

(2) $\mathrm{R}$ is a minimal non-empty union of $\mathrm{X}$-bridges of $\mathrm{M}$.

(3) $R$ is non-empty and is minimal with respect to $B_{1} \sqcup B_{X} \sqcup B_{2} \in \mathcal{B}(M)$ whenever $\mathrm{B}_{\mathrm{X}} \in \mathcal{B}(\mathrm{M} \mid \mathrm{X})$ is extended by $\mathrm{B}_{1}$ to a basis of $\mathrm{M} \mid(\mathrm{R} \sqcup \mathrm{X})$ and (independently) extended by $\mathrm{B}_{2}$ to a basis of $\mathrm{M} \mid(\mathrm{E}-\mathrm{R})$.

If $M$ has finite rank, these conditions are also equivalent to:

(4) $\mathrm{R}$ is non-empty and is minimal with respect to $r(\mathrm{R} \sqcup \mathrm{X})-\mathrm{r}(\mathrm{X})+\mathrm{r}(\mathrm{E}-\mathrm{R})=$ $r(M)$.

The next corollary is a special case of Corollary 5.1.9.

Corollary 5.1.10. Let $\mathrm{M}$ be an independence space on $\mathrm{E}$ and let $\mathrm{K} \subseteq \mathrm{E}$. Then the following are equivalent: 
(1) $\mathrm{K}$ is a connected component of $\mathrm{M}$.

(2) $\mathrm{K}$ is a minimal non-empty separator of $\mathrm{M}$.

(3) $\mathrm{K}$ is non-empty and is minimal with respect to $\mathrm{B}_{\mathrm{K}} \sqcup \mathrm{B}_{\mathrm{E}-\mathrm{K}} \in \mathcal{B}(M)$ for every $\mathrm{B}_{\mathrm{K}} \in \mathcal{B}(\mathrm{M} \mid \mathrm{K})$ and every $\mathrm{B}_{\mathrm{E}-\mathrm{K}} \in \mathcal{B}(\mathrm{M} \mid(\mathrm{E}-\mathrm{K}))$.

(4) $\mathrm{K}$ is non-empty and is minimal with respect to $\mathrm{B} \cap \mathrm{K} \in \mathcal{B}(\mathrm{M} \mid \mathrm{K})$ and $\mathrm{B}-\mathrm{K} \in$ $\mathcal{B}(M \mid(E-K))$ for every $B \in \mathcal{B}(M)$.

If $\mathrm{M}$ has finite rank, these conditions are also equivalent to:

(5) $\mathrm{K}$ is non-empty and is minimal with respect to $\mathrm{r}(\mathrm{K})+\mathrm{r}(\mathrm{E}-\mathrm{K})=\mathrm{r}(\mathrm{M})$.

As $B \in \mathcal{B}(M[\mathcal{D}])$ if and only if $\ell[B] \in \mathcal{B}(D)$ for every configuration $\mathcal{D}=(D, E, \ell)$, we have the following consequence of Corollary 5.1.9.(3).

Proposition 5.1.11. Let $\mathcal{D}=(\mathrm{D}, \mathrm{E}, \mathrm{\ell})$ be a configuration, let $\mathrm{X} \subseteq \mathrm{E}$ and let $\mathrm{R} \subseteq \mathrm{E}-\mathrm{X}$. Then $\mathrm{R}$ is an $\mathrm{X}$-bridge of $\mathcal{D}$ if and only if $\ell[\mathrm{R}]$ is an $\ell[\mathrm{X}]$-bridge of $\mathrm{D}$.

So it suffices in the context of bridges to simply view configurations as subsets of their ambient vector spaces. The next result is used in Section 6.1 .

Proposition 5.1.12. Let $\mathrm{D}$ be a configuration, let $\mathrm{X} \subseteq \mathrm{D}$ and let $\Re \subseteq \mathrm{D}-\mathrm{X}$. Then $\mathfrak{R}$ is a union of $\mathrm{X}$-bridges of $\mathrm{D}$ if and only if

$$
\langle\Re \sqcup X\rangle \cap\langle\mathrm{D}-\mathfrak{R}\rangle=\langle\mathrm{X}\rangle .
$$

Proof. Suppose that $\mathfrak{R}$ is a union of X-bridges of D. Clearly $\langle\mathrm{X}\rangle \subseteq\langle\mathfrak{R} \sqcup$ $X\rangle \cap\langle D-\mathfrak{R}\rangle$. If this containment were strict and $B_{X} \in \mathcal{B}(X)$ were extended by $B_{1}$ to a basis of $\Re \sqcup X$ and (independently) extended by $B_{2}$ to a basis of $D-\Re$, then $B_{1} \sqcup B_{X} \sqcup B_{2}$ would be linearly dependent, contradicting Proposition 5.1.4. Hence equality holds.

Noe suppose that $\langle\mathfrak{R} \sqcup \mathrm{X}\rangle \cap\langle\mathrm{D}-\mathfrak{R}\rangle=\langle\mathrm{X}\rangle$. Let $\mathrm{B}_{\mathrm{X}} \in \mathcal{B}(\mathrm{X})$. Extend $\mathrm{B}_{\mathrm{X}}$ by $B_{1}$ to a basis of $\Re \sqcup X$ and (independently) by $B_{2}$ to a basis of $D-\Re$. 
Clearly $B:=B_{1} \sqcup B_{X} \sqcup B_{2}$ spans $D$. If $B \notin \mathcal{B}(D)$, then there would exist non-zero $y \in\left\langle B_{X} \sqcup B_{1}\right\rangle \cap\left\langle B_{2}\right\rangle$. But then $y \in\left\langle B_{X}\right\rangle$, contradicting the linear independence of $B_{X} \sqcup B_{2}$. Hence $B \in \mathcal{B}(D)$.

Combining this result with Corollaries 5.1.9 and 5.1.10 and Proposition 5.1.11 yields the next two corollaries.

Corollary 5.1.13. Let $\mathrm{D}$ be a configuration, let $\mathrm{X} \subseteq \mathrm{D}$ and let $\mathrm{R} \subseteq \mathrm{D}-\mathrm{X}$. Then the following are equivalent:

(1) $\mathrm{R}$ is an $\mathrm{X}$-bridge of $\mathrm{D}$.

(2) $\mathrm{R}$ is a minimal non-empty union of $\mathrm{X}$-bridges of $\mathrm{D}$.

(3) $\mathrm{R}$ is non-empty and is minimal with respect to $\mathrm{B}_{1} \sqcup \mathrm{B}_{\mathrm{X}} \sqcup \mathrm{B}_{2} \in \mathcal{B}(\mathrm{D})$ whenever $\mathrm{B}_{\mathrm{X}} \in \mathcal{B}(\mathrm{X})$ is extended by $\mathrm{B}_{1}$ to a basis of $\mathrm{R} \sqcup \mathrm{X}$ and (independently) extended by $\mathrm{B}_{2}$ to a basis of $\mathrm{D}-\mathrm{R}$.

(4) $\mathrm{R}$ is non-empty and is minimal with respect to $\langle\mathrm{R} \sqcup \mathrm{X}\rangle \cap\langle\mathrm{D}-\mathrm{R}\rangle=\langle\mathrm{X}\rangle$.

If $\mathrm{D}$ is finite-dimensional, these conditions are also equivalent to:

(5) $R$ is non-empty and is minimal with respect to $\operatorname{dim}(R \sqcup X)-\operatorname{dim}(X)+$ $\operatorname{dim}(D-R)=\operatorname{dim}(D)$.

Corollary 5.1.14. Let $\mathrm{D}$ be a configuration and let $\mathrm{K} \subseteq \mathrm{D}$. Then the following are equivalent:

(1) $\mathrm{K}$ is a connected component of $\mathrm{D}$.

(2) $\mathrm{K}$ is a minimal non-empty separator of $\mathrm{D}$.

(3) $\mathrm{K}$ is non-empty and is minimal with respect to $\mathrm{B}_{\mathrm{K}} \sqcup \mathrm{B}_{\mathrm{D}-\mathrm{K}} \in \mathcal{B}(\mathrm{D})$ for every $\mathrm{B}_{\mathrm{K}} \in \mathcal{B}(\mathrm{K})$ and every $\mathrm{B}_{\mathrm{D}-\mathrm{K}} \in \mathcal{B}(\mathrm{D}-\mathrm{K})$.

(4) $\mathrm{K}$ is non-empty and is minimal with respect to $\mathrm{B} \cap \mathrm{K} \in \mathcal{B}(\mathrm{K})$ and $\mathrm{B}-\mathrm{K} \in$ $\mathcal{B}(\mathrm{D}-\mathrm{K})$ for every $\mathrm{B} \in \mathcal{B}(\mathrm{D})$. 
(5) $\mathrm{K}$ is non-empty and is minimal with respect to $\langle\mathrm{D}\rangle=\langle\mathrm{K}\rangle \oplus\langle\mathrm{D}-\mathrm{K}\rangle$.

If $\mathrm{D}$ is finite-dimensional, these conditions are also equivalent to:

(6) $\mathrm{K}$ is non-empty and is minimal with respect to $\operatorname{dim}(\mathrm{K})+\operatorname{dim}(\mathrm{D}-\mathrm{K})=$ $\operatorname{dim}(\mathrm{D})$.

We now derive some further results concerning bridges, the first of which follows immediately from Proposition 5.1.2.

Corollary 5.1.15. Let $\mathrm{M}$ be an independence space on $\mathrm{E}$ and let $\mathrm{X} \subseteq \mathrm{E}$. Then the $\mathrm{X}$-bridges of $\mathrm{M}$ partition $\mathrm{E}-\mathrm{X}$. In particular, the connected components of $M$ partition $\mathrm{E}$.

Corollary 5.1.16. Let $\mathrm{D}$ be a configuration and let $\mathrm{X} \subseteq \mathrm{D}$. Then the $\mathrm{X}$-bridges of $\mathrm{D}$ partition $\mathrm{D}-\mathrm{X}$. In particular, the connected components of $\mathrm{D}$ partition $\mathrm{D}$.

Proposition 5.1.17. Let $M$ be an independence space and let $X \subseteq \mathrm{E}(\mathrm{M})$. Then each X-bridge $\mathrm{R}$ of $\mathrm{M}$ either has the form $\mathrm{cl}(\mathrm{R})-\mathrm{cl}(\mathrm{X})$ or is a singleton containing an element of $\mathrm{cl}(\mathrm{X})-\mathrm{X}$. In particular, each connected component $\mathrm{K}$ of $\mathrm{M}$ either has the form $\mathrm{cl}(\mathrm{K})-\mathcal{L}(\mathrm{M})$ or is a singleton consisting of a loop of $\mathrm{M}$.

Proof. Let $e \in E(M)-X$. If $e \in \mathcal{L}(M / X)$, then $\gamma_{M / X}(e)=\{e\}$ and $e \in$ $\mathrm{cl}_{M}(X)-X$. So suppose not. Clearly $\gamma_{M / X}(e) \subseteq \operatorname{cl}_{M}\left(\gamma_{M / X}(e)\right)-\operatorname{cl}_{M}(X)$. Let $f \in \mathrm{cl}_{M}\left(\gamma_{M / X}(e)\right)-\mathrm{cl}_{M}(X)$. Then $f \in \mathrm{cl}_{M / X}\left(\gamma_{M / X}(e)\right)$ by (CL2) and Proposition 3.6.8. Consequently either $f \in \gamma_{M / X}(e)$, or by (3.5.1) there exists $C \in \mathcal{C}(M / X)$ containing both $f$ and some $g \in \gamma_{M / X}(e)$. In the latter case, $f \in \gamma_{M / X}(g)$ by definition, so $f \in \gamma_{M / X}(e)$ by Proposition 5.1.2. Hence $\gamma_{M / X}(e)=\mathrm{cl}_{M}\left(\gamma_{M / X}(e)\right)-\mathrm{cl}_{M}(X)$. The observation for connected components of $M$ follows on setting $X=\varnothing$.

Corollary 5.1.18. Let $\mathrm{D}$ be a configuration and let $\mathrm{X} \subseteq \mathrm{D}$. Then each $\mathrm{X}$-bridge $\mathrm{R}$ of $\mathrm{D}$ either has the form $\mathrm{D} \cap(\langle\mathrm{R}\rangle-\langle\mathrm{X}\rangle)$ or is a singleton containing an element of $\mathrm{D} \cap(\langle\mathrm{X}\rangle-\mathrm{X})$. In particular, each connected component $\mathrm{K}$ of $\mathrm{D}$ either has the form $\mathrm{D} \cap(\langle\mathrm{K}\rangle-\{\mathbf{0}\})$ or is $\{\mathbf{0}\}$. 
The following results involving closures are worth noting.

Proposition 5.1.19. Let $M$ be an independence space, let $X \subseteq E(M)$ and let $\Re$ be a union of $\mathrm{X}$-bridges of $\mathrm{M}$. Then

$$
\mathrm{cl}(\mathfrak{R} \sqcup \mathrm{X})=\mathfrak{R} \cup \mathrm{cl}(\mathrm{X})
$$

Proof. By (CL1) and Lemma 3.5.3,

$$
\Re \cup \operatorname{cl}(\mathrm{X}) \subseteq \operatorname{cl}(\Re \cup \operatorname{cl}(\mathrm{X}))=\operatorname{cl}(\Re \sqcup \mathrm{X})
$$

So let $e \in \operatorname{cl}(\Re \sqcup X)$ and suppose that $e \notin \Re \cup \operatorname{cl}(X)$. Then there exists $\mathrm{C} \in \mathcal{C}(M)$ such that $e \in C \subseteq \Re \sqcup X \sqcup\{e\}$. In light of (C4), we may assume that $|C-X|$ is minimal without loss of generality. If $C \subseteq X \sqcup\{e\}$, then $e \in \operatorname{cl}(X)$, so $C \cap \Re$ is non-empty. Let $f \in C \cap \Re$. Then $e \in \gamma_{M / X}(f)$ by Proposition 3.6.6, contradicting $e \notin \Re$. Hence $\operatorname{cl}(\Re \sqcup X) \subseteq \Re \cup \operatorname{cl}(X)$.

Corollary 5.1.20. Let $M$ be an independence space and let $\mathfrak{K}$ be a separator of M. Then

$$
\operatorname{cl}(\mathfrak{K})=\mathfrak{K} \cup \mathcal{L}(M) .
$$

Corollary 5.1.21. Let $\mathrm{D}$ be a configuration and let $\mathrm{X} \subseteq \mathrm{D}$. Then for every union $\Re$ of $\mathrm{X}$-bridges of $\mathrm{D}$,

$$
\mathrm{D} \cap\langle\mathfrak{R} \sqcup \mathrm{X}\rangle=\mathfrak{R} \cup(\mathrm{D} \cap\langle\mathrm{X}\rangle)
$$

In particular, for every separator $\mathfrak{K}$ of $\mathrm{D}$,

$$
\mathrm{D} \cap\langle\mathfrak{K}\rangle=\mathfrak{K} \cup(\mathrm{D} \cap\{\mathbf{0}\})
$$

Proposition 5.1.22. Let $M$ be an independence space, let $X \subseteq E(M)$ and let $\mathfrak{R}_{1}$ and $\mathfrak{R}_{2}$ be unions of $\mathrm{X}$-bridges of $\mathrm{M}$ containing no common $\mathrm{X}$-bridge of $\mathrm{M}$. Then

$$
\mathrm{cl}\left(\Re_{1} \sqcup \mathrm{X}\right) \cap \mathrm{cl}\left(\Re_{2} \sqcup \mathrm{X}\right)=\mathrm{cl}(\mathrm{X})
$$


Proof.

$$
\begin{array}{rlrl}
\operatorname{cl}\left(\Re_{1} \sqcup \mathrm{X}\right) \cap \operatorname{cl}\left(\Re_{2} \sqcup \mathrm{X}\right) & =\left(\Re_{1} \cup \operatorname{cl}(\mathrm{X})\right) \cap\left(\Re_{2} \cup \mathrm{cl}(\mathrm{X})\right) & & \text { by Prop. 5.1.19 } \\
& =\left(\Re_{1} \cap \mathfrak{R}_{2}\right) \cup \mathrm{cl}(\mathrm{X}) & \\
& =\operatorname{cl}(\mathrm{X}) & & \text { by Coroll. 5.1.15. }
\end{array}
$$

Corollary 5.1.23. Let $M$ be an independence space and let $\mathfrak{K}_{1}$ and $\mathfrak{K}_{2}$ be separators of $M$ containing no common connected component of $M$. Then

$$
\operatorname{cl}\left(\mathfrak{K}_{1}\right) \cap \operatorname{cl}\left(\mathfrak{K}_{2}\right)=\mathcal{L}(M) .
$$

Corollary 5.1.24. Let $\mathrm{D}$ be a configuration and let $\mathrm{X} \subseteq \mathrm{D}$. Then whenever $\mathfrak{R}_{1}$ and $\mathfrak{R}_{2}$ are unions of $\mathrm{X}$-bridges of $\mathrm{D}$ containing no common $\mathrm{X}$-bridge of $\mathrm{D}$,

$$
\mathrm{D} \cap\left\langle\mathfrak{R}_{1} \sqcup \mathrm{X}\right\rangle \cap\left\langle\mathfrak{R}_{2} \sqcup \mathrm{X}\right\rangle=\mathrm{D} \cap\langle\mathrm{X}\rangle
$$

In particular, whenever $\mathfrak{K}_{1}$ and $\mathfrak{K}_{2}$ are separators of $\mathrm{D}$ containing no common connected component of $\mathrm{D}$,

$$
\mathrm{D} \cap\left\langle\mathfrak{K}_{1}\right\rangle \cap\left\langle\mathfrak{K}_{2}\right\rangle=\mathrm{D} \cap\{\mathbf{0}\}
$$

Proposition 5.1.25. Let $\mathrm{M}$ be an independence space on $\mathrm{E}$, let $\mathrm{X} \subseteq \mathrm{E}$ and let $\Re$ be a union of $\mathrm{X}$-bridges of $\mathrm{M}$. Then

$$
\operatorname{cl}(\Re) \cap \operatorname{cl}(E-\Re) \subseteq \operatorname{cl}(X)
$$

Proof. Immediate from Corollary 5.1.15 and Proposition 5.1.22.

Corollary 5.1.26. Let $M$ be an independence space on $\mathrm{E}$ and let $\mathfrak{K}$ be a separator of $M$. Then

$$
\operatorname{cl}(\mathfrak{K}) \cap \operatorname{cl}(E-\mathfrak{K}) \subseteq \mathcal{L}(M)
$$


Note that the converse of the last result does not hold (consider a partition of a 4-circuit into two 2-element sets).

Corollary 5.1.27. Let $\mathrm{D}$ be a configuration and let $\mathrm{X} \subseteq \mathrm{D}$. Then for every union $\Re$ of $\mathrm{X}$-bridges of $\mathrm{D}$,

$$
\mathrm{D} \cap\langle\mathfrak{R}\rangle \cap\langle\mathrm{D}-\mathfrak{R}\rangle \subseteq \mathrm{D} \cap\langle\mathrm{X}\rangle
$$

In particular, for every separator $\mathfrak{K}$ of $\mathrm{D}$,

$$
\mathrm{D} \cap\langle\mathfrak{K}\rangle \cap\langle\mathrm{D}-\mathfrak{K}\rangle \subseteq \mathrm{D} \cap\{\mathbf{0}\}
$$

\subsection{Roundness}

This section treats round independence spaces, which generalise complete graphs. While complete graphs vary only in size, round independence spaces also vary in form-a consequence of the greater generality of the matroidal setting.

Definition 5.2.1. A split of an independence space $M$ on $E$ is a set $\left\{F, F^{\prime}\right\}$ where $F, F^{\prime} \in \mathcal{F}(M)-\{E\}$ are such that $F \cup F^{\prime}=E$. The order of a split $\left\{F, F^{\prime}\right\}$ is $\lambda_{M}\left(F, F^{\prime}\right)$ if $M$ has finite rank, and undefined otherwise. A split is a k-split if it has order $k$. An independence space splits if it has a split, and is non-splitting or round otherwise.

A split of a configuration $\mathcal{D}$ is a split of $M[\mathcal{D}]$. The order of a split $\left\{F, F^{\prime}\right\}$ of $\mathcal{D}$ is $\lambda_{\mathcal{D}}\left(F, F^{\prime}\right)$. The notions of $k$-split, splits, non-splitting and round are then defined as for independence spaces.

The terminology "splits" first appears in [KK82], while the terminology "round" first appears in [GW03]. As our interest is in those independence spaces and configurations that do not split, we shall predominantly use the positively-phrased "round."

Clearly every independence space (configuration) having rank (dimension) at most 1 is trivially round. Round independence spaces may 
be characterised as in the next proposition, the first equivalence of which was proven for matroids on pp. 11-12 of [Kab06].

Proposition 5.2.2. Let $\mathrm{M}$ be an independence space on $\mathrm{E}$. Then the following are equivalent:

(1) $M$ is round.

(2) $\widetilde{M}$ is round.

(3) $\mathrm{E}-\mathrm{H}$ is spanning for every $\mathrm{H} \in \mathcal{H}(\mathrm{M})$.

Proof. The equivalence of (1) and (2) follows from the fact that $E=F^{\prime} \cup F$ for some $F, F^{\prime} \in \mathcal{F}(M)-\{E\}$ if and only if $E(\widetilde{M})=F \cup F^{\prime}$ for some $F$, $F^{\prime} \in \mathcal{F}(\widetilde{M})-E(\widetilde{M})$. So we demonstrate the equivalence of (1) and (3).

Suppose that $M$ is round. Let $H \in \mathcal{H}(M)$ and let $F=\operatorname{cl}(E-H)$. Then $E=H \cup F$, so as $H \neq E$ and $M$ is round, $F=E$. Consequently $E-H$ is spanning.

Now suppose that $E-H$ is spanning for every $H \in \mathcal{H}(M)$. Let $F$, $F^{\prime} \in \mathcal{F}(M)$ be such that $F \cup F^{\prime}=E$ and suppose that $F$ is proper. Then there exists $H \in \mathcal{H}(M)$ containing $F$. But then $E-H \subseteq E-F \subseteq F^{\prime}$ is spanning, so $\mathrm{E}=\mathrm{cl}(\mathrm{E}-\mathrm{H}) \subseteq \mathrm{cl}\left(\mathrm{F}^{\prime}\right)=\mathrm{F}^{\prime} \subseteq \mathrm{E}$. Hence $\mathrm{F}^{\prime}=\mathrm{E}$, and so $\mathrm{M}$ is round.

If $\mathrm{M}$ were a matroid, $\mathrm{E}-\mathrm{H}$ in Proposition 5.2.2.(3) would be a cocircuit-that is, a circuit of the dual matroid $M^{*}$ of $M$ (see pp. 68-70 of [Ox104]). We cannot make such an identification for arbitrary $M$, as the class of independence spaces does not possess a reasonable notion of duality (see pp. 260-261 of [Ox178]).

Recall that a hyperplane of a vector space $\mathrm{U}$ is a maximal proper subspace of $U$. Then we have the following characterisation of round configurations.

Corollary 5.2.3. Let $\mathcal{D}$ be a configuration having point set $\mathrm{D}$. Then the following are equivalent: 
(1) $\mathcal{D}$ is round.

(2) D is round.

(3) $\widetilde{\mathcal{D}}$ is round.

(4) $\mathrm{D}$ is not contained in the union of any pair of proper subspaces of $\langle\mathrm{D}\rangle$.

(5) $\langle\mathrm{D}-\mathrm{H}\rangle=\langle\mathrm{D}\rangle$ for every hyperplane $\mathrm{H}$ of $\langle\mathrm{D}\rangle$.

As a consequence of the first equivalence in Corollary 5.2.3, without loss of generality we may simply view configurations as subsets of their ambient vector spaces for the remainder of this section.

The next corollary follows immediately from the fact that no vector space is contained in the union of a pair of its proper subspaces.

Corollary 5.2.4. Vector spaces are round. Additionally, $\mathrm{M}[\mathrm{V}]$ is round for every vector space $\mathrm{V}$.

The following proposition provides a sufficient condition for when roundness of a minor certifies roundness. It was proved for matroids on pp. 11-12 of [Kab06].

Proposition 5.2.5. Every independence space having a round spanning restriction is round.

Proof. Let $M$ be an independence space on $E$ and let $X \subseteq E$ be spanning and such that $M \mid X$ is round. Suppose that $M$ splits. Then $E=F \cup F^{\prime}$ for some $F, F^{\prime} \in \mathcal{F}(M)-\{E\}$. Now,

$$
X \cap \operatorname{cl}(F \cap X) \subseteq X \cap \operatorname{cl}(F)=X \cap F \subseteq X
$$

So $F \cap X \in \mathcal{F}(M \mid X)$ by Corollary 3.5.6. If $F \cap X=X$, then

$$
\mathrm{F} \subseteq \mathrm{E}=\mathrm{cl}(\mathrm{X})=\mathrm{cl}(\mathrm{F} \cap \mathrm{X}) \subseteq \mathrm{cl}(\mathrm{F})=\mathrm{F}
$$


contradicting $F \neq E$. Thus $F \cap X \neq X$. By symmetry, $F^{\prime} \cap X \in \mathcal{F}(M \mid X)-$ $\{X\}$ also. As $X=(F \cap X) \cup\left(F^{\prime} \cap X\right)$, it follows that $M \mid X$ splits, which is impossible. Hence $M$ is round.

Corollary 5.2.6. Every configuration D containing round $\mathrm{N}$ such that $\langle\mathrm{N}\rangle=$ $\langle\mathrm{D}\rangle$ is round.

In light of Proposition 5.2.5, the next result provides a condition under which roundness of an independence space can be certified by roundness of a submatroid. This is particularly useful when a finite certificate is necessary.

Proposition 5.2.7. Every finite-rank round independence space has a finite round spanning restriction.

Proof. Let $M$ be a finite-rank round independence space on $E$ and let $\mathrm{X} \subseteq \mathrm{E}$ be finite, spanning and lexicographically minimal with respect to

$$
s(X)=\left(s_{0}(X), \ldots, s_{r(M)-2}(X)\right)
$$

where $s_{k}(X)$ is the number of $k$-splits of $M \mid X$ for each $k$.

Suppose that $M \mid X$ splits. Let $k$ be the least order for which $M \mid X$ has a split and let $\left\{X_{1}, X_{2}\right\}$ be a k-split of $M \mid X$.

As $M$ is round, $\left\{\operatorname{cl}_{M}\left(X_{1}\right), \operatorname{cl}_{M}\left(X_{2}\right)\right\}$ is not a split of $M$, so there exists $e \in E-\left(\operatorname{cl}_{M}\left(X_{1}\right) \cup \mathrm{cl}_{M}\left(X_{2}\right)\right)$. Let $X^{\prime}=X \sqcup\{e\}$. Clearly $X^{\prime}$ spans $M$ and is finite. We argue that $s\left(X^{\prime}\right)<s(X)$, thus establishing the result by contradiction.

(1) $\left\{\mathrm{F}_{1}^{\prime}-\{e\}, \mathrm{F}_{2}^{\prime}-\{e\}\right\}$ is a split of $\mathrm{M} \mid \mathrm{X}$ having order at most $\mathrm{k}$ whenever $\left\{\mathrm{F}_{1}^{\prime}, \mathrm{F}_{2}^{\prime}\right\}$ is a split of $\mathrm{M} \mid \mathrm{X}^{\prime}$ having order at most $\mathrm{k}$.

Let $\left\{F_{1}^{\prime}, F_{2}^{\prime}\right\}$ be a split of $M \mid X^{\prime}$ having order at most $k$. As $F_{1}^{\prime} \in \mathcal{F}\left(M \mid X^{\prime}\right)$, 
it follows from Corollary 3.5.6 that

$$
\begin{aligned}
\mathrm{X}^{\prime} \cap \mathrm{cl}_{M}\left(\mathrm{~F}_{1}^{\prime}\right) & \subseteq \mathrm{F}_{1}^{\prime} \subseteq \mathrm{X} \\
\left(\mathrm{X}^{\prime}-\{e\}\right) \cap \mathrm{cl}_{M}\left(\mathrm{~F}_{1}^{\prime}-\{e\}\right) & \subseteq \mathrm{F}_{1}^{\prime}-\{e\} \subseteq \mathrm{X}^{\prime}-\{e\} \quad \text { by }(\mathrm{CL} 2) \\
\mathrm{X} \cap \mathrm{cl}_{M}\left(\mathrm{~F}_{1}^{\prime}-\{e\}\right) & \subseteq \mathrm{F}_{1}^{\prime}-\{e\} \subseteq \mathrm{X} .
\end{aligned}
$$

Thus $F_{1}^{\prime}-\{e\} \in \mathcal{F}(M \mid X)$ by Corollary 3.5.6. If $F_{1}^{\prime}-\{e\}=X$, then

$$
\begin{array}{rlrl}
X^{\prime} & =X^{\prime} \cap \mathrm{cl}_{M}(X) & \\
& =X^{\prime} \cap \operatorname{cl}_{M}\left(F_{1}^{\prime}-\{e\}\right) & \\
& =\operatorname{cl}_{M \mid X^{\prime}}\left(F_{1}^{\prime}-\{e\}\right) & & \text { by Prop. 3.5.5 } \\
& \subseteq \mathrm{cl}_{M \mid X^{\prime}}\left(F_{1}^{\prime}\right) & & \text { by (CL2) } \\
& =F_{1}^{\prime} & & \\
& \subseteq X^{\prime} &
\end{array}
$$

-a contradiction. Thus $\mathrm{F}_{1}^{\prime}-\{\mathrm{e}\} \neq \mathrm{X}$. It follows by symmetry that $\mathrm{F}_{2}^{\prime}-\{e\} \in \mathcal{F}(\mathrm{M} \mid \mathrm{X})-\{\mathrm{X}\}$ also, and so $\left\{\mathrm{F}_{1}^{\prime}-\{e\}, \mathrm{F}_{2}^{\prime}-\{e\}\right\}$ is a split of $M \mid X$. Finally,

$$
\begin{aligned}
\lambda_{M \mid X}\left(F_{1}^{\prime}-\{e\}, F_{2}^{\prime}-\{e\}\right)= & r_{M \mid X}\left(F_{1}^{\prime}-\{e\}\right)+r_{M \mid X}\left(F_{2}^{\prime}-\{e\}\right) \\
& -r(M \mid X) \\
= & r_{M}\left(F_{1}^{\prime}-\{e\}\right)+r_{M}\left(F_{2}^{\prime}-\{e\}\right)-r_{M}(X) \\
\leqslant & r_{M}\left(F_{1}^{\prime}\right)+r_{M}\left(F_{2}^{\prime}\right)-r_{M}\left(X^{\prime}\right) \\
= & r_{M \mid X^{\prime}}\left(F_{1}^{\prime}\right)+r_{M \mid X^{\prime}}\left(F_{2}^{\prime}\right)-r\left(M \mid X^{\prime}\right) \\
= & \lambda_{M \mid X^{\prime}}\left(F_{1}^{\prime}, F_{2}^{\prime}\right) .
\end{aligned}
$$

The claim follows.

So as $M \mid X$ has no splits having order at most $k-1$, neither does $M \mid X^{\prime}$. If $M \mid X^{\prime}$ has no $k$-splits, then clearly $s\left(X^{\prime}\right)<s(X)$. So suppose otherwise.

(2) Whenever $\left\{\mathrm{F}_{1}, \mathrm{~F}_{2}\right\}$ is a $\mathrm{k}$-split of $\mathrm{M} \mid \mathrm{X}$, there exists at most one $\mathrm{k}$-split $\left\{\mathrm{F}_{1}^{\prime}, \mathrm{F}_{2}^{\prime}\right\}$ 
of $M \mid X^{\prime}$ such that $\left\{F_{1}^{\prime}-\{e\}, F_{2}^{\prime}-\{e\}\right\}=\left\{F_{1}, F_{2}\right\}$.

For suppose that there are two distinct $k$-splits of $M \mid X^{\prime}$ satisfying the hypothesis of (2). Then these are some pair of $\left\{F_{1}, F_{2} \sqcup\{e\}\right\},\left\{F_{1} \sqcup\{e\}, F_{2}\right\}$ and $\left\{F_{1} \sqcup\{e\}, F_{2} \sqcup\{e\}\right\}$. Consequently there exists $i$ for which $F_{i}, F_{i} \sqcup$ $\{e\} \in \mathcal{F}\left(M \mid X^{\prime}\right)$, and so $r_{M}\left(F_{i} \sqcup\{e\}\right)=r_{M}\left(F_{i}\right)+1$. But then there exists $j$ such that $r_{M}\left(F_{j}^{\prime}\right)=r_{M}\left(F_{j}^{\prime}-\{e\}\right)+1$, where $\left\{F_{1}^{\prime}, F_{2}^{\prime}\right\}$ is one of the pair of $k$-splits of $M \mid X^{\prime}$. Thus

$$
\begin{aligned}
k & =\lambda_{M \mid X}\left(F_{1}, F_{2}\right) \\
& =r_{M \mid X}\left(F_{1}\right)+r_{M \mid X}\left(F_{2}\right)-r(M \mid X) \\
& =r_{M}\left(F_{1}\right)+r_{M}\left(F_{2}\right)-r_{M}(X) \quad \text { as } X \text { spans } M \\
& =r_{M}\left(F_{1}\right)+r_{M}\left(F_{2}\right)-r_{M}\left(X^{\prime}\right) \quad \\
& <r_{M}\left(F_{1}\right)+r_{M}\left(F_{2}\right)-r_{M}\left(X^{\prime}\right)+1 \\
& \leqslant r_{M}\left(F_{1}^{\prime}\right)+r_{M}\left(F_{2}^{\prime}\right)-r_{M}\left(X^{\prime}\right) \\
& =r_{M \mid X^{\prime}}\left(F_{1}^{\prime}\right)+r_{M \mid X^{\prime}}\left(F_{2}^{\prime}\right)-r\left(M \mid X^{\prime}\right) \\
& =\lambda_{M \mid X^{\prime}}\left(F_{1}^{\prime}, F_{2}^{\prime}\right) \\
& =k
\end{aligned}
$$

—a contradiction. The claim follows.

Consequently $M \mid X^{\prime}$ has at most as many $k$-splits as $M \mid X$. So as $\left\{X_{1}, X_{2}\right\}$ is a k-split of $M \mid X$ not obtainable from a k-split of $M \mid X^{\prime}$ by deleting e, $M \mid X^{\prime}$ has strictly fewer $k$-splits than $M \mid X$. As both have no splits of order at most $k-1$, it follows that $s\left(X^{\prime}\right)<s(X)$, completing the proof.

Corollary 5.2.8. Every finite-dimensional round configuration $\mathrm{D}$ contains finite round $\mathrm{N}$ such that $\langle\mathrm{N}\rangle=\langle\mathrm{D}\rangle$.

The following result was proved for matroids on pp. 11-12 of [Kab06].

Proposition 5.2.9. Every contraction of a round independence space is round. 
Proof. Let $M$ be a round independence space on $E$, let $X \subseteq E$ and suppose that $M / X$ splits. Then $E-X=F \cup F^{\prime}$ for some $F, F^{\prime} \in \mathcal{F}(M / X)-\{E-X\}$. Consequently $E=(X \sqcup F) \cup\left(X \sqcup F^{\prime}\right)$. It then follows from Corollary 3.6.9 that $X \sqcup F, X \sqcup F^{\prime} \in \mathcal{F}(M)-\{E\}$, contradicting the roundness of $M$. Hence $M / X$ is round.

Corollary 5.2.10. Every contraction of a round configuration is round.

Proof. Let $\mathrm{D}$ be a round configuration and let $\mathrm{X} \subseteq \mathrm{D}$. As $\mathrm{D}$ is round, $\mathrm{M}[\mathrm{D}]$ is round. Proposition 5.2.9 shows that $(M[D]) / X$ is round, so $(M[D / X]) \backslash X$ is round by Proposition 4.4.5. But then $M[D / X]$ is round by Proposition 5.2.5, so $\mathrm{D} / \mathrm{X}$ is round.

Recall that a triangle is a circuit of size 3, and for every set I, the collection of all 2-element subsets of I is denoted by $[\mathrm{I}]^{2}$.

It is reasonable to ask which frequently encountered classes of independence spaces are round. One such class are the Dowling cliques, which were introduced for matroids in [GK06].

Definition 5.2.11. A Dowling clique is an independence space $M$ having $E(M)=B \sqcup X$, where $B=\left\{b_{i} \mid i \in I\right\} \in \mathcal{B}(M)$ and $X=\left\{x_{J} \mid J \in[I]^{2}\right\}$ are such that $\left\{b_{i}, b_{j}, x_{\{i, j\}}\right\}$ is a triangle whenever $i, j \in I$ are distinct. The joints of $M$ are the elements of $B$.

A configuration $D$ is a Dowling clique if $M[D]$ is a Dowling clique. The joints of $\mathrm{D}$ are the joints of $\mathrm{M}[\mathrm{D}]$.

The next proposition was proved for finite Dowling cliques on p. 13 of [Kab06].

Proposition 5.2.12. Dowling cliques are round.

Proof. Let $\mathrm{M}$ be a Dowling clique on $\mathrm{E}$ with set of joints $\mathrm{B}$ and let $\mathrm{X}=\mathrm{E}-$ B. Suppose that $M$ splits. Then $E=F_{1} \cup F_{2}$ for some $F_{1}, F_{2} \in \mathcal{F}(M)-\{E\}$. For each $i$, let $B_{i}=B \cap F_{i}$. Then as $F_{i} \neq E$, necessarily $B_{i} \neq B$. So $B_{i}-B_{3-i}$ is non-empty. Let $b_{i} \in B_{i}-B_{3-i}$. Then there exists $x \in X$ such that 
$\left\{b_{1}, b_{2}, x\right\}$ is a triangle. If $x \in F_{i}$, then $b_{3-i} \in F_{i}$, and thus $b_{3-i} \in B_{i}$, which is impossible. Consequently $x \notin F_{1} \cup F_{2}$, contradicting $F_{1} \cup F_{2}=E$. Hence $M$ is round.

Dowling cliques arise from complete graphs. Recall that a graph is complete if every distinct pair of its vertices are linked by exactly one edge. A clique is a complete subgraph of a graph. On considering the set of edges incident on some fixed vertex of a complete graph, it is easily seen that:

Proposition 5.2.13. Let $\mathrm{K}$ be a complete graph. Then $\mathrm{M}(\mathrm{K})$ is a Dowling clique. In particular, it is round.

Consequently (graph) cliques give rise to Dowling cliques, and so round subindependence spaces generalise (graph) cliques. This relationship later helps motivate the formulation chosen for a matroidal notion of chordality in Section 5.3.

Another class of round independence spaces is a subclass of the uniform independence spaces, which are introduced below.

Definition 5.2.14. Let $m \in \omega$, let $\beta \geqslant m$ be a cardinal and let $E$ be any set having cardinality $\beta$. Then the uniform independence space of rank $m$ on $\beta$ elements, denoted $U_{m, \beta}$, is $(E,\{X \subseteq E:|X| \leqslant m\})$.

Proposition 5.2.15. Let $m \in \omega$ and let $\beta \geqslant m$ be a cardinal. Then $U_{m, \beta}$ is an independence space that is defined uniquely up to isomorphism.

Proof. Let $\mathrm{U}_{\mathrm{m}, \mathrm{E}}=(\mathrm{E},\{\mathrm{X} \subseteq \mathrm{E}:|\mathrm{X}| \leqslant \mathrm{m}\})$ for every set $\mathrm{E}$ having cardinality $\beta$. Clearly $U_{m, E}$ satisfies (I1)-(I3), so we establish (I4). Let $X \subseteq E$ be such that $Y \in \mathcal{J}_{\mathfrak{m}, E}$ for every finite $Y \subseteq X$. Then $X$ contains no $(m+1)$-element subsets, so $X \in \mathcal{J}_{\mathfrak{m}, E}$ and (I4) holds.

Whenever $E$ and $E^{\prime}$ are two sets having cardinality $\beta$, there exists a bijection $\phi: E \rightarrow E^{\prime}$, and clearly $X \in \mathcal{J}_{\mathfrak{m}, \mathrm{E}}$ if and only if $\phi[X] \in \mathcal{J}_{\mathfrak{m}, E^{\prime}}$. Consequently $\mathrm{U}_{\mathrm{m}, \mathrm{E}} \cong \mathrm{U}_{\mathrm{m}, \mathrm{E}^{\prime}}$. 
On allowing infinite $m$ in Definition 5.2.14, two possibilities arise. If $m=\beta$, a class of isomorphic free independence spaces is obtained, while if $m<\beta$, the definition of independence fails to satisfy (I4). Consequently only finite values of $m$ are considered in Definition 5.2.14.

The next result was noted for matroids in [GGW03].

Proposition 5.2.16. Let $\mathrm{m} \in \omega$ and let $\beta \geqslant m$ be a cardinal. Then $\mathrm{U}_{\mathrm{m}, \beta}$ is round if and only if $\beta \geqslant 2 m-1$.

Proof. If $m=0$, then $U_{m, \beta}$ is trivially round and clearly $\beta \geqslant 2 m-1$. So suppose that $m \geqslant 1$. The hyperplanes of $U_{m, \beta}$ are precisely its ( $m-$ $1)$-element sets. If $\beta \geqslant 2 m-1$, the complements of these each contain at least $m$ elements and thus span, while if $\beta<2 m-1$, the complements each contain at most $m-1$ elements, and thus do not span. The result then follows from Proposition 5.2.2.(3).

Considering $\left\{U_{m+1,2 m+1} \mid m \in \omega\right\}$, we see that there exist round matroids of arbitrarily high rank having no non-trivial proper round restrictions - a consequence of the sparsity of these matroids.

Further material on round matroids may be found in [Kun86].

\subsection{Chordal Saturation}

This section develops an analogue of another concept from graph theory. Given a graph $G$ and a cycle $C$ of $G$, a chord of $C$ is an edge $e \in E(G)-E(C)$ such that $e \subseteq V(C)$ (see Figure 5.2). A graph is chordal (or triangulated) if each of its cycles has a chord.

Chordality plays a fundamental role in Kříž and Thomas' proof of Theorem 1.1.6 in [KT91]. They use it to formulate a locally verifiable property certifying when a graph is globally tree-like. This is necessary for a subsequent application of Rado's Selection Lemma. As our aim is to establish an analogue of Theorem 1.1.6 for configurations (namely Theorem 7.1.1), it is natural to investigate chordality for configurations. 


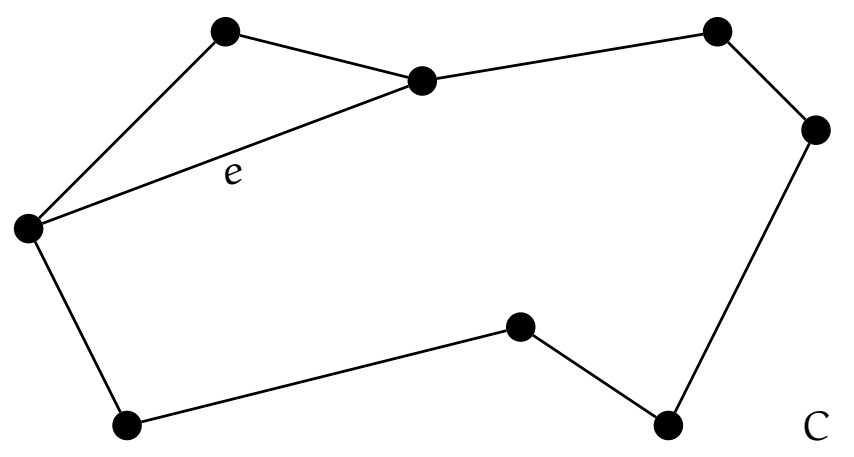

Figure 5.2: A cycle C having chord e.

While chordality is relatively well-understood for graphs, little work has been done on matroidal chordality. This may be partly due to the difficulty of formulating a universally applicable definition: different objectives have resulted in different definitions-see [BG86, CFK04], [dMV03, BdM04] and [Hli05] for three of these. The approach taken here is closest to that of [Hli05], which is also motivated by tree-decompositions.

Recall that the clique number of a graph $\mathrm{G}$, denoted $\omega(\mathrm{G})$, is

$$
\omega(G)=\sup \{|K|: K \text { is a finite clique of } G\} .
$$

A chordal graph having finite clique number is a tree-like union of cliques. That is, it has a tree-decomposition in which each bag induces a clique (see pp. 88-89 of [KT91]). As round subindependence spaces generalise cliques, this suggests that a reasonable notion of "chordal configuration" might be "a tree-like union of round subconfigurations." Formally, we would define a configuration to be "chordal" if its restriction to any bag of some fixed tree-decomposition was round.

The difficulty with this is a potential lack of density: two round subconfigurations $N$ and $N^{\prime}$ having $\langle N\rangle \cap\left\langle N^{\prime}\right\rangle \neq\{\mathbf{0}\}$ may share few points, or even be disjoint (see Figure 5.3). In contrast, the intersection of two cliques $K$ and $K^{\prime}$ in a chordal graph is another clique on $V(K) \cap V\left(K^{\prime}\right)$ - 
there is no drop in "dimension."

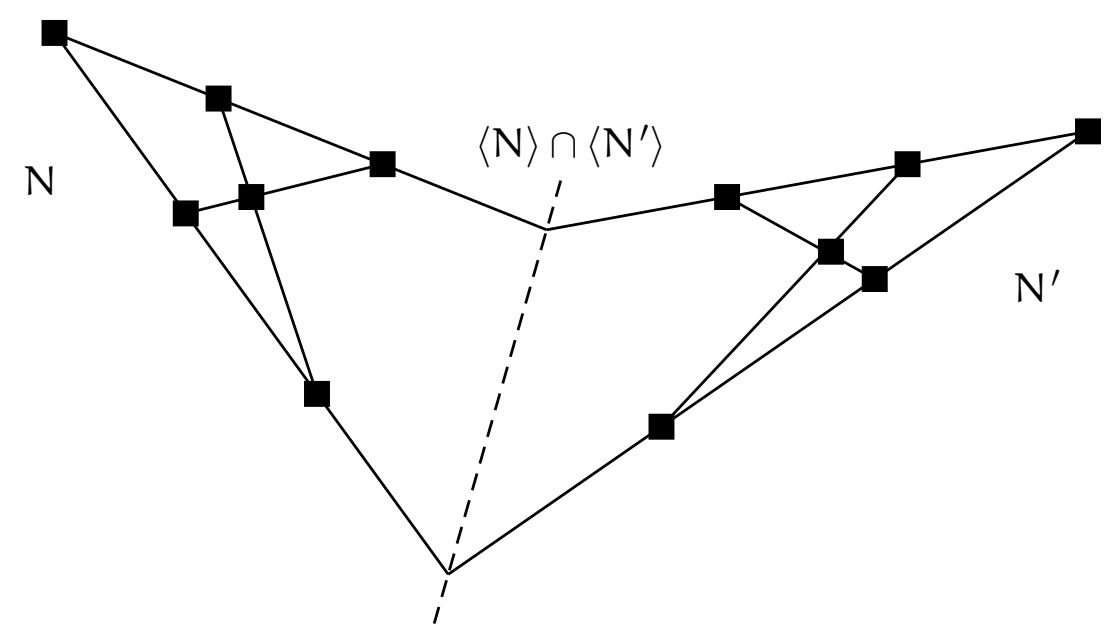

Figure 5.3: A geometric representation of a configuration containing disjoint copies $N$ and $N^{\prime}$ of $M\left(K_{4}\right)$ for which $\operatorname{dim}\left(\langle N\rangle \cap\left\langle N^{\prime}\right\rangle\right)=2$.

The configuration's ambient vector space can be exploited to overcome this difficulty by imposing the requirement that all maximal round subconfigurations be entire subspaces.

Definition 5.3.1. Let $\mathcal{D}=(D, E, \ell)$ be a configuration and let:

(CS1) (Chordality) Whenever $\mathrm{C} \in \mathcal{C}(\mathcal{D})$ has size at least 4, there exist distinct $c, c^{\prime} \in C$ and $e \in E$ such that $\left\{c, c^{\prime}, e\right\},\left(C-\left\{c, c^{\prime}\right\}\right) \sqcup\{e\} \in \mathcal{C}(\mathcal{D})$.

(CS2) (Saturation) $\langle\mathrm{N}\rangle \subseteq \mathrm{D}$ for every round $\mathrm{N} \subseteq \mathrm{D}$.

Then $\mathcal{D}$ is chordal if Chordality holds, saturated if Saturation holds and chordally saturated if both hold.

The following proposition is easy to establish.

Proposition 5.3.2. Let $\mathcal{D}$ be a configuration having point set $\mathrm{D}$. Then

(1) $\mathcal{D}$ is saturated if and only if $\mathrm{D}$ is saturated, and 
(2) $\mathcal{D}$ is chordal if and only if $\mathrm{D}$ is chordal.

Consequently there is no loss of generality in simply viewing configurations as subsets of their ambient vector spaces for the remainder of this section.

Proposition 5.3.3. Every chordally saturated configuration is a non-empty union of subspaces. In particular, it contains $\mathbf{0}$.

Proof. Let $\mathrm{D}$ be a chordally saturated configuration and let $\mathrm{d} \in \mathrm{D}$. Then $\{\mathrm{d}\}$ is round, so $\langle\mathrm{d}\rangle \subseteq \mathrm{D}$ by Saturation. Consequently $\mathrm{D}$ is a union of subspaces. To see that this union is non-empty, observe that $\varnothing$ is round, so $\langle\varnothing\rangle=\{\mathbf{0}\} \subseteq \mathrm{D}$ by Saturation.

Chordal saturation is not usually preserved by unions or intersections.

Example 5.3.4. Let $\left\{b_{1}, b_{2}, b_{3}, b_{4}\right\}$ be a basis for a 4 -dimensional vector space.

(1) Let $\mathrm{D}=\left\langle\mathrm{b}_{1}\right\rangle \cup\left\langle\mathrm{b}_{2}\right\rangle \cup\left\langle\mathrm{b}_{1}+\mathrm{b}_{2}\right\rangle \cup\left\langle\mathrm{b}_{3}\right\rangle \cup\left\langle\mathrm{b}_{4}\right\rangle \cup\left\langle\mathrm{b}_{1}+\mathrm{b}_{3}+\mathrm{b}_{4}\right\rangle$. The maximal subspaces contained in $\mathrm{D}$ are trivially chordally saturated. While $\mathrm{D}$ contains a Dowling clique having joints $\mathrm{b}_{1}$ and $\mathrm{b}_{2},\left\langle\mathrm{~b}_{1}, \mathrm{~b}_{2}\right\rangle \nsubseteq \mathrm{D}$, so $D$ is not saturated. The circuit $\left\{b_{1}, b_{3}, b_{4}, b_{1}+b_{3}+b_{4}\right\}$ certifies that $D$ is not chordal.

(2) Let $D_{1}=\left\langle b_{1}, b_{2}\right\rangle \cup\left\langle b_{1}+b_{2}, b_{3}\right\rangle$ and let $D_{2}=\left\langle b_{1}, b_{3}\right\rangle \cup\left\langle b_{1}+b_{3}, b_{2}\right\rangle$. Clearly each $D_{i}$ is chordally saturated. However, $D_{1} \cap D_{2}=\left\langle b_{1}\right\rangle \cup$ $\left\langle b_{2}\right\rangle \cup\left\langle b_{3}\right\rangle \cup\left\langle b_{1}+b_{2}+b_{3}\right\rangle$ is not chordal for the same reason as $D$ in (1).

However, Saturation is easily seen to be preserved under intersection.

Proposition 5.3.5. Every intersection of saturated V-configurations is saturated.

Chordal saturation is preserved on "restricting to a vector space." 
Proposition 5.3.6. Let $\mathrm{D}$ be a chordally saturated $\mathrm{V}$-configuration and let $\mathrm{U} \leqslant$ $\mathrm{V}$. Then $\mathrm{D} \cap \mathrm{U}$ is chordally saturated.

Proof. Let $\mathrm{C} \in \mathcal{C}(\mathrm{D} \cap \mathrm{U})$ have size at least 4 . Then $\mathrm{C} \in \mathcal{C}(\mathrm{D})$, so by Chordality for $D$ there exist distinct $c, c^{\prime} \in C$ and $d \in D$ such that $\left\{c, c^{\prime}, d\right\}$, $\left(C-\left\{c, c^{\prime}\right\}\right) \sqcup\{d\} \in \mathcal{C}(D)$. As $d \in\left\langle\left\{c, c^{\prime}\right\}\right\rangle$, clearly $d \in U$. Consequently $\left\{c, c^{\prime}, d\right\},\left(C-\left\{c, c^{\prime}\right\}\right) \sqcup\{d\} \in \mathcal{C}(D \cap U)$, and so Chordality holds for $D \cap U$.

Now let $\mathrm{N} \subseteq \mathrm{D} \cap \mathrm{U}$ be round. Then $\langle\mathrm{N}\rangle \subseteq \mathrm{D}$ by Saturation for $\mathrm{D}$, so $\langle\mathrm{N}\rangle \subseteq \mathrm{D} \cap \mathrm{U}$. Hence Saturation holds for $\mathrm{D} \cap \mathrm{U}$.

It is well known that finite chordal graphs may be characterised as those graphs that can be constructed recursively by pasting along cliques, starting from complete graphs (see p. 127 of [Die06]). We now work towards a similar characterisation for finite-dimensional chordally saturated configurations, starting with the following proposition.

Proposition 5.3.7. Let $\mathrm{D}$ be a chordally saturated configuration and let $\mathrm{V}, \mathrm{W} \leqslant$ $\langle\mathrm{D}\rangle$ be such that $\mathrm{D} \subseteq \mathrm{V} \cup \mathrm{W}, \mathrm{V}=\langle\mathrm{D} \cap(\mathrm{V}-\mathrm{W})\rangle$ and $\mathrm{W}=\langle\mathrm{D} \cap(\mathrm{W}-\mathrm{V})\rangle$. Then $\mathrm{V} \cap \mathrm{W} \subseteq \mathrm{D}$.

Proof. It follows from Proposition 5.3.3 that $\mathbf{0} \in \mathrm{D}$. Consequently the result holds if $\mathrm{V} \cap \mathrm{W}=\{\boldsymbol{0}\}$. So suppose otherwise. Let $z \in \mathrm{V} \cap \mathrm{W}$ and suppose that $z \notin D$. Then $z$ is non-zero and there exists minimum-sized $\mathrm{X}=\left\{\mathrm{x}_{1}, \ldots, \mathrm{x}_{\mathrm{m}}\right\} \subseteq \mathrm{D} \cap(\mathrm{V}-\mathrm{W})$ such that $\mathrm{X} \sqcup\{z\}$ is linearly dependent. Similarly, there exists minimum-sized $Y=\left\{y_{1}, \ldots, y_{n}\right\} \subseteq D \cap(W-V)$ such that $\mathrm{Y} \sqcup\{z\}$ is linearly dependent. So there exist non-zero scalars $\mu_{1}$, $\ldots, \mu_{m}, \xi_{1}, \ldots, \xi_{n}$ such that

$$
z=\sum_{i=1}^{m} \mu_{i} x_{i}=\sum_{j=1}^{n} \xi_{j} y_{j} .
$$

It follows that $X \sqcup Y$ is linearly dependent, and so it contains some $C \in$ $\mathcal{C}(D)$. As $X$ is independent, some $y_{j} \in C$. If $|C \cap Y|=1$, then $y_{j} \in$ 
$\left\langle C-\left\{y_{j}\right\}\right\rangle \subseteq V$, contradicting $y_{j} \notin V$. Consequently $|C \cap Y| \geqslant 2$. Similarly, $|\mathrm{C} \cap X| \geqslant 2$.

It follows that $|C| \geqslant 4$. So by Chordality, $D$ contains a linear combination of a pair of distinct elements of $C$. The choices of $V, W, X$ and $Y$ ensure that these elements both belong to $V$ without loss of generality. It follows from Saturation that their span is contained in $\mathrm{D}$, contradicting the minimality of $\mathrm{m}$. Hence $z \in \mathrm{D}$, and so $\mathrm{V} \cap \mathrm{W} \subseteq \mathrm{D}$.

Corollary 5.3.8. Let $\mathrm{D}$ be a chordally saturated configuration and let $\mathrm{V}, \mathrm{W} \leqslant$ $\langle\mathrm{D}\rangle$ be such that $\mathrm{D} \subseteq \mathrm{V} \cup \mathrm{W}$ and $\mathrm{V} \cap \mathrm{W}$ is minimal. Then $\mathrm{V} \cap \mathrm{W} \subseteq \mathrm{D}$.

Proof. Let $\mathrm{V}^{\prime}=\langle\mathrm{D} \cap \mathrm{V}\rangle$ and let $\mathrm{W}^{\prime}=\langle\mathrm{D} \cap \mathrm{W}\rangle$. Then $\mathrm{V}^{\prime}, \mathrm{W}^{\prime} \leqslant\langle\mathrm{D}\rangle$ are such that $\mathrm{D} \subseteq \mathrm{V}^{\prime} \cup \mathrm{W}^{\prime}$ and $\mathrm{V}^{\prime} \cap \mathrm{W}^{\prime} \subseteq \mathrm{V} \cap \mathrm{W}$. It follows from the minimality of $\mathrm{V} \cap \mathrm{W}$ that $\mathrm{V}^{\prime} \cap \mathrm{W}^{\prime}=\mathrm{V} \cap \mathrm{W}$.

Let $V_{1}^{\prime}=\left\langle D \cap\left(V^{\prime}-W^{\prime}\right)\right\rangle$ and suppose that $V^{\prime} \neq V_{1}^{\prime}$. Then there exists non-zero $V_{2}^{\prime} \leqslant\left\langle D \cap V^{\prime} \cap W^{\prime}\right\rangle$ such that $V^{\prime}=V_{1}^{\prime} \oplus V_{2}^{\prime}$. But then $V_{1}^{\prime}$, $W^{\prime} \leqslant\langle D\rangle$ are such that $\mathrm{D} \subseteq \mathrm{V}_{1}^{\prime} \cup \mathrm{W}^{\prime}$ and $\mathrm{V}_{1}^{\prime} \cap \mathrm{W}^{\prime} \subsetneq \mathrm{V}^{\prime} \cap \mathrm{W}^{\prime}=\mathrm{V} \cap \mathrm{W}$, contradicting the minimality of $\mathrm{V} \cap \mathrm{W}$. Thus $\mathrm{V}^{\prime}=\mathrm{V}_{1}^{\prime}$. Similarly, $\mathrm{W}^{\prime}=$ $\left\langle\mathrm{D} \cap\left(\mathrm{W}^{\prime}-\mathrm{V}^{\prime}\right)\right\rangle$, and so $\mathrm{V} \cap \mathrm{W}=\mathrm{V}^{\prime} \cap \mathrm{W}^{\prime} \subseteq \mathrm{D}$ by Proposition 5.3.7.

Definition 5.3.9. Let $\mathcal{D}, \mathcal{D}_{1}$ and $\mathcal{D}_{2}$ be configurations and let $D_{i}$ be the point set of $\mathcal{D}_{i}$ for each $i$. Then $\mathcal{D}$ arises by pasting $\mathcal{D}_{1}$ and $\mathcal{D}_{2}$ together along $\mathrm{D}_{1} \cap \mathrm{D}_{2}$ if $\mathcal{D}=\mathcal{D}_{1} \cup \mathcal{D}_{2}$ and $\left\langle\mathrm{D}_{1}\right\rangle \cap\left\langle\mathrm{D}_{2}\right\rangle \subseteq \mathrm{D}_{1} \cap \mathrm{D}_{2}$.

Note that whenever $\mathcal{D}$ arises by pasting $\mathcal{D}_{1}$ and $\mathcal{D}_{2}$ together along $D_{1} \cap D_{2}$, it is necessarily the case that $0 \in D_{1} \cap D_{2}$.

Proposition 5.3.10. Every finite-dimensional chordally saturated configuration can be constructed recursively by pasting along subspaces, starting from subspaces.

Proof. Let D be a finite-dimensional chordally saturated configuration. Suppose that $\mathrm{D}$ is not a subspace and that all chordally saturated configurations having strictly smaller dimension can be constructed recursively as stated. Then by Saturation there exist $\mathrm{V}, \mathrm{W} \lessgtr\langle\mathrm{D}\rangle$ such that 
$\mathrm{D} \subseteq \mathrm{V} \cup \mathrm{W}$. Without loss of generality, $\mathrm{V} \cap \mathrm{W}$ is minimal. It follows from Proposition 5.3.6 that each of $\mathrm{D} \cap \mathrm{V}$ and $\mathrm{D} \cap \mathrm{W}$ is chordally saturated, and so can be constructed recursively as stated. Moreover, $\mathrm{V} \cap \mathrm{W} \subseteq \mathrm{D}$ by Corollary 5.3.8, so $\mathrm{D}$ arises by pasting $\mathrm{D} \cap \mathrm{V}$ and $\mathrm{D} \cap \mathrm{W}$ together along the subspace $\mathrm{V} \cap \mathrm{W}$.

The converse of this result also holds. It is derived in Proposition 6.4.3. We now examine the preservation of chordal saturation under contraction.

Proposition 5.3.11. Every contraction of a chordal configuration is chordal.

Proof. Let $\mathrm{D}$ be a chordal configuration, let $\mathrm{X} \subseteq \mathrm{D}$ and let $\pi$ determine a contraction of $\mathrm{D}$ by $X$. Let $C=\left\{c_{1}, \ldots, c_{m}\right\} \subseteq D$ having $m \geqslant 4$ be such that $\pi[C] \in \mathcal{C}(\pi[D])$ and $\pi\left(c_{i}\right) \neq \pi\left(c_{j}\right)$ whenever $i \neq j$. Then there exist non-zero scalars $\mu_{1}, \ldots, \mu_{m}$ such that

$$
\begin{aligned}
\sum_{i=1}^{m} \mu_{i} \pi\left(c_{i}\right) & =0 \\
\sum_{i=1}^{m} \mu_{i} c_{i} & \in\langle X\rangle .
\end{aligned}
$$

So there exists $C^{\prime}=\left\{c_{1}^{\prime}, \ldots, c_{n}^{\prime}\right\} \subseteq X$ such that $C \sqcup C^{\prime} \in \mathcal{C}(D)$ and

$$
\sum_{i=1}^{m} \mu_{i} c_{i}+\sum_{j=1}^{n} \xi_{j} c_{j}^{\prime}=\mathbf{0}
$$

for some non-zero scalars $\xi_{1}, \ldots, \xi_{n}$. Re-indexing without loss of generality, either $c^{*}:=\mu_{1} c_{1}+\mu_{2} c_{2} \in D$ or $c_{1}^{\prime \prime}:=\mu_{1} c_{1}+\xi_{1} c_{1}^{\prime} \in D$ by Chordality. In the first case, $\pi\left[\left\{c_{1}, c_{2}, c^{*}\right\}\right], \pi\left[\left\{c^{*}, c_{3}, \ldots, c_{m}\right\}\right] \in \mathcal{C}(\pi[D])$, so Chordality holds. In the second, as $\pi\left(c_{1}^{\prime \prime}\right)=\pi\left(c_{1}\right)$, we may redefine $c_{1}:=c_{1}^{\prime \prime}$, effectively reducing $\left|C^{\prime}\right|$ by 1 . As $\left|C^{\prime}\right| \in \omega$ initially, it follows by induction that the first case must be realised. 
Proposition 5.3.12. Let $\pi$ determine a contraction of a chordally saturated configuration $\mathrm{D}$ some by finite-dimensional $\mathrm{X}$. Then for every round $\mathrm{N}^{\prime} \subseteq \pi[\mathrm{D}]$, there exists round $\mathrm{N} \subseteq \mathrm{D}$ such that $\mathrm{N}^{\prime}=\pi[\mathrm{N}]$. In particular, $\pi[\mathrm{D}]$ is saturated.

Proof. In light of Proposition 4.4.4.(2), it suffices to establish the result for $X=\{x\} \in \mathcal{J}(D)$. So let $U=\left\langle N^{\prime}\right\rangle \oplus\langle x\rangle$. It follows from Proposition 5.3.6 that $\mathrm{D} \cap \mathrm{U}$ is chordally saturated. If $\mathrm{D} \cap \mathrm{U}$ is round, then the result follows on setting $\mathrm{N}=\mathrm{D} \cap \mathrm{U}$. So suppose otherwise.

Then there exist $\mathrm{V}, \mathrm{W} \subseteq \mathrm{U}$ such that $\mathrm{D} \cap \mathrm{U} \subseteq \mathrm{V} \cup \mathrm{W}$. Without loss of generality, $\mathrm{V}=\langle\mathrm{D} \cap(\mathrm{V}-\mathrm{W})\rangle$ and $\mathrm{W}=\langle\mathrm{D} \cap(\mathrm{W}-\mathrm{V})\rangle$. So as $\mathrm{D} \cap \mathrm{U}$ is chordally saturated, $\mathrm{V} \cap \mathrm{W} \subseteq \mathrm{D} \cap \mathrm{U}$ by Proposition 5.3.7.

If $x \in \mathrm{V} \cap \mathrm{W}$, then $\mathrm{N}^{\prime}$ would split. Thus $x \notin \mathrm{V} \cap \mathrm{W}$. Without loss of generality, $x \in V-W$. As $N^{\prime}$ does not split, $W$ is a hyperplane of $\mathrm{U}$. Consequently both $W$ and $\left\langle\mathrm{N}^{\prime}\right\rangle$ are complements of $\langle x\rangle$ in $\mathrm{U}$ (see Figure 5.4).

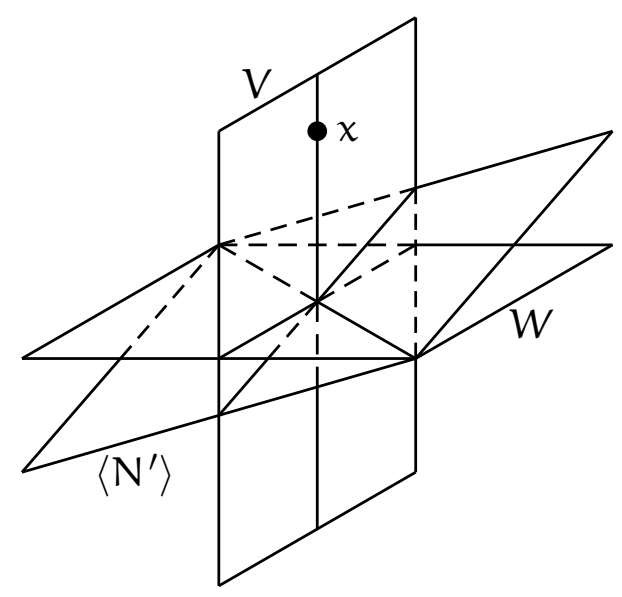

Figure 5.4: Proof of Proposition 5.3.12.

As $\mathrm{V} \cap W \subseteq \mathrm{D}$, it follows that every point of $\mathrm{N}^{\prime}$ is the $\pi$-image of some point in $\mathrm{D} \cap \mathrm{W}$. So there exists round $\mathrm{N} \subseteq \mathrm{D} \cap \mathrm{W}$ such that $\mathrm{N}^{\prime}=\pi[\mathrm{N}]$. Finally, $\langle\mathrm{N}\rangle \subseteq \mathrm{D}$ by Saturation, so $\left\langle\mathrm{N}^{\prime}\right\rangle \subseteq \pi[\mathrm{D}]$. 
Combining Propositions 5.3.11 and 5.3.12 gives the next corollary.

Corollary 5.3.13. Every contraction of a chordally saturated configuration by a finite-dimensional set is chordally saturated.

Proposition 5.3.14. Let $\pi$ determine a contraction of a chordally saturated configuration $\mathrm{D}$. Then for every finite-dimensional round $\mathrm{N}^{\prime} \subseteq \pi[\mathrm{D}]$, there exists round $\mathrm{N} \subseteq \mathrm{D}$ such that $\mathrm{N}^{\prime}=\pi[\mathrm{N}]$. In particular, $\left\langle\mathrm{N}^{\prime}\right\rangle \subseteq \pi[\mathrm{D}]$.

Proof. Let $\pi$ determine a contraction of $D$ by $X$. If $\operatorname{dim}(X) \in \omega$, then the result follows from Proposition 5.3.12. So suppose that $\operatorname{dim}(X)=\infty$. Then by Corollary 5.2.8 there exists finite round $N_{0}^{\prime} \subseteq N^{\prime}$ such that $\left\langle N_{0}^{\prime}\right\rangle=$ $\left\langle N^{\prime}\right\rangle$. It follows from Proposition 4.4.7 that there exists $\pi^{\prime}$ determining a contraction of $\mathrm{D}$ by some finite $\mathrm{X}_{0} \subseteq \mathrm{X}$ such that $\mathrm{N}_{0}^{\prime} \subseteq \pi^{\prime}[\mathrm{D}]$ and $\pi=\pi^{\prime \prime} \circ \pi^{\prime}$ for some projection $\pi^{\prime \prime}$ fixing $\operatorname{im}(\pi)$. So by Proposition 5.3.12, there exists round $\mathrm{N}_{0} \subseteq \mathrm{D}$ such that $\mathrm{N}_{0}^{\prime}=\pi^{\prime}\left[\mathrm{N}_{0}\right]$. As $\pi^{\prime \prime}$ fixes $\operatorname{im}(\pi) \supseteq \mathrm{N}_{0}^{\prime}$, it follows that $\mathrm{N}_{0}^{\prime}=\pi\left[\mathrm{N}_{0}\right]$. So by Saturation,

$$
\begin{aligned}
\mathrm{D} & \supseteq\left\langle\mathrm{N}_{0}\right\rangle \\
\pi[\mathrm{D}] & \supseteq \pi\left[\left\langle\mathrm{N}_{0}\right\rangle\right] \\
& =\left\langle\pi\left[\mathrm{N}_{0}\right]\right\rangle \\
& =\left\langle\mathrm{N}_{0}^{\prime}\right\rangle \\
& =\left\langle\mathrm{N}^{\prime}\right\rangle .
\end{aligned}
$$

Hence there exists $N \subseteq\left\langle N_{0}\right\rangle$ containing $N_{0}$ such that $N^{\prime}=\pi[N]$. Corollary 5.2.6 shows that $\mathrm{N}$ is round.

Combining Propositions 5.3.11 and 5.3.14 gives the following corollary.

Corollary 5.3.15. Let $\mathrm{D}$ be a chordally saturated configuration and let $\mathrm{X} \subseteq \mathrm{D}$ be such that every round subconfiguration of $\mathrm{D} / \mathrm{X}$ is finite-dimensional. Then $\mathrm{D} / \mathrm{X}$ is chordally saturated. 


\section{Chapter 6}

\section{Tree-Decompositions of Configurations}

This chapter discusses tree-decompositions of configurations. Some elementary results are given first, and then the main theorem is established for the special case of finite configurations. This result is lifted to infinite configurations after appropriate development of the relationships between round and chordally saturated configurations and tree-decompositions.

\subsection{Definitions and Elementary Results}

Intuitively, a tree-decomposition of an object (such as a graph, hypergraph, matroid or configuration) is a decomposition of that object into parts of bounded size such that the parts fit together in a tree-like way. The size of the largest part-called the width of the decompositionindicates how tree-like the decomposition is. When the width is small, the decomposition is very tree-like, and vice versa.

Given a notion of tree-decomposition for a particular class of objects, it can be determined how tree-like an object of that class is by finding the least width among its tree-decompositions (if any). This is the object's 
tree-width.

Tree-decompositions and tree-width were first introduced in this sense as part of the Graph Minors Project of Robertson and Seymour. Robertson and Seymour defined both for finite graphs in [RS84], the third article in a series of 23 . These definitions were subsequently generalised to finite rooted hypergraphs in [RS90]. It was only a matter of time until tree-decompositions of infinite graphs were considered. They were first treated in [RST91] and have subsequently been investigated by Thomas in particular.

Given the tremendous success of the theory of tree-decompositions of graphs, it was natural to try to develop a similar theory for matroids. It is not immediately apparent that this is possible, as the definition of treedecomposition given in [RS84] relies heavily on vertices, which have no obvious matroidal analogue. However, Geelen observed that an equivalent definition could be formulated using only edges, thus opening the way forward. As a result, definitions for both matroids and finite configurations were given by Hliněný and Whittle in [HW06].

The latter of these gives rise to Definition 6.1.2. It is convenient to first introduce some further terminology. Recall that we write $t t^{\prime}$ for the edge of a tree incident on vertices $t$ and $t^{\prime}$ (if it exists).

Definition 6.1.1. Let $U$ be a vector space and let $T$ be a tree. Whenever $\sigma: \mathrm{V}(\mathrm{T}) \rightarrow \mathcal{S}(\mathrm{U})$, we shall extend $\sigma$ to $\mathrm{V}(\mathrm{T}) \sqcup \mathrm{E}(\mathrm{T})$ by defining

$$
\sigma(e)=\sigma(t) \cap \sigma\left(t^{\prime}\right)
$$

for every $e=t t^{\prime} \in E(T)$. The subspaces displayed by $t \in V(T)$ are

$$
\sum_{t^{\prime} \in V\left(T_{i}\right)} \sigma\left(t^{\prime}\right) \quad(i \in I)
$$

where $\left\{T_{i} \mid i \in I\right\}$ are the connected components of $T-\{t\}$. Similarly, the 
subspaces displayed by $e \in \mathrm{E}(\mathrm{T})$ are

$$
\sum_{t \in V\left(T_{i}\right)} \sigma(t) \quad(i=1,2)
$$

where $T_{1}$ and $T_{2}$ are the connected components of $T-\{e\}$. Finally, the subspace associated with $\mathrm{G} \leqslant \mathrm{T}$ is

$$
\sum_{t \in V(G)} \sigma(t)
$$

Replacing these sums by unions yields the sets displayed by $\mathrm{t}$, the sets displayed by $e$ and the set associated with $G$ respectively.

Definition 6.1.2. Let $\mathcal{D}=(\mathrm{D}, \mathrm{E}, \ell)$ be a U-configuration. Then a tree-decomposition of $\mathcal{D}$ is a pair $(T, \sigma)$, where $\mathrm{T}$ is a tree and $\sigma: \mathrm{V}(\mathrm{T}) \rightarrow \mathcal{S}(\mathrm{U})$, satisfying:

(TD1) $\mathrm{D} \subseteq \bigcup_{\mathrm{t} \in \mathrm{V}(\mathrm{T})} \sigma(\mathrm{t})$.

(TD2) (Interpolation) $S_{1} \cap S_{2} \subseteq \sigma(e)$ for every e $\in E(T)$, where $S_{1}$ and $S_{2}$ are the subspaces displayed by $e$.

The vertex bags of $(T, \sigma)$ are

$$
\sigma(t) \quad(t \in V(T))
$$

and the width of $\mathrm{t} \in \mathrm{V}(\mathrm{T})$, denoted $w(\mathrm{t})$, is $\operatorname{dim}(\sigma(\mathrm{t}))$. Similarly, the edge bags of $(T, \sigma)$ are

$$
\sigma(e) \quad(e \in \mathrm{E}(\mathrm{T}))
$$

and the width of $e \in \mathrm{E}(\mathrm{T})$, denoted $w(e)$, is $\operatorname{dim}(\sigma(e))$. The width of $(\mathrm{T}, \sigma)$ is

$$
\sup \{w(t) \mid t \in V(T)\}
$$

and the tree-width of $\mathcal{D}$, denoted $\operatorname{tw}(\mathcal{D})$, is the infimum of the widths of the tree-decompositions of $\mathcal{D}$. A tree-decomposition of $\mathcal{D}$ is optimal if it 
has width $\operatorname{tw}(\mathcal{D})$, and finite if its tree is finite. We denote the collection of all tree-decompositions of $\mathcal{D}$ by $\mathcal{T} \mathcal{D}(\mathcal{D})$.

Given a configuration $\mathcal{D}$ having point set $\mathrm{D}$, it is always possible to form a trivial tree-decomposition $(T, \sigma)$ of $\mathcal{D}$ by setting

(1) $\mathrm{T}=(\{t\}, \varnothing)$, and

(2) $\sigma:\{t\} \rightarrow \mathcal{S}(\langle\mathrm{D}\rangle): t \mapsto\langle\mathrm{D}\rangle$.

In contrast, some definitions of tree-decomposition for other classes of object admit the possibility that an object may not have a tree-decomposition. For example, the uncountable complete graph $K_{\boldsymbol{N}_{1}}$ has no (graph) tree-decomposition (see p. 5 of [Ad106]).

The statement (TD1) is a non-triviality axiom requiring that a configuration be covered by each of its tree-decompositions. The Interpolation axiom forces all of the connectivity between the "sides" of the configuration displayed by an edge to "pass through" that edge's bag (see Figure 6.1). This ensures that the decomposition has a tree-like structure. Note that this requirement frequently results in a bag $\sigma(x)$ being strictly larger than the span $\langle\sigma(x) \cap D\rangle$ of those points of the configuration that it contains.

As a configuration's point set contains each of its subconfigurations' point sets, we have the following unsurprising result.

Proposition 6.1.3. Let $\mathcal{D}$ be a configuration and let $\mathcal{D}^{\prime} \leqslant \mathcal{D}$. Then $\mathcal{T} \mathcal{D}(\mathcal{D}) \subseteq$ $\mathcal{T} \mathcal{D}\left(\mathcal{D}^{\prime}\right)$. In particular, $\operatorname{tw}(\mathcal{D}) \geqslant \operatorname{tw}\left(\mathcal{D}^{\prime}\right)$.

Alternately taking $\mathcal{D}^{\prime}=\mathrm{D}$ and $\mathcal{D}^{\prime}=\widetilde{\mathcal{D}}$ in Proposition 6.1.3 (where D is the point set of $\mathcal{D}$ ) gives the next corollary.

Corollary 6.1.4. Let $\mathcal{D}$ be a configuration having point set $\mathrm{D}$. Then $\mathcal{T} \mathcal{D}(\mathcal{D})=$ $\mathcal{T D}(\mathrm{D})=\mathcal{T} \mathcal{D}(\widetilde{\mathcal{D}})$. In particular, $\operatorname{tw}(\mathcal{D})=\operatorname{tw}(\mathrm{D})=\operatorname{tw}(\widetilde{\mathcal{D}})$. 


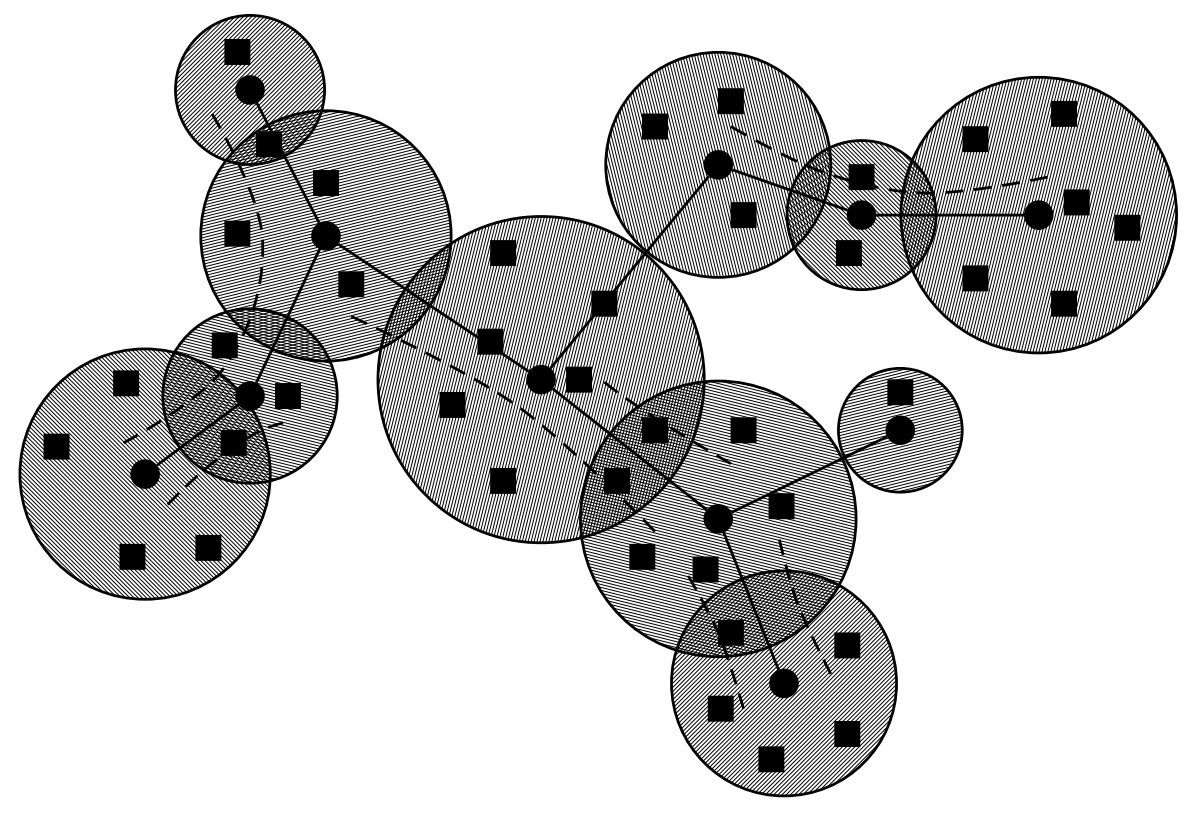

Figure 6.1: A sketch of a tree-decomposition of a configuration. Large discs, squares and dashed lines are intended to suggest vertex bags, configuration points and connectivity respectively.

In light of Corollary 6.1.4, without loss of generality we may simply view configurations as subsets of their ambient vector spaces for the remainder of this section.

Motivated by an observation regarding tree-decompositions of graphs, we now derive an alternative characterisation of Interpolation. Like a tree-decomposition of a configuration, a tree-decomposition of a graph has an interpolation property requiring that all of the graph's connectivity $^{1}$ "pass through" the decomposition's bags in a manner consistent with the decomposition's tree structure. In particular, deleting a (vertex) bag separates the "branches" of the decomposition (see Figure 6.2). Moreover, if it did not, there would be a path between two distinct "branches" of the decomposition not passing through the bag of interest-that is, interpo-

\footnotetext{
${ }^{1}$ Now concretely embodied by paths.
} 
lation would fail. So interpolation corresponds precisely to the property of "cracking" a tree-decomposition of a graph on a (vertex) bag always separating the decomposition.
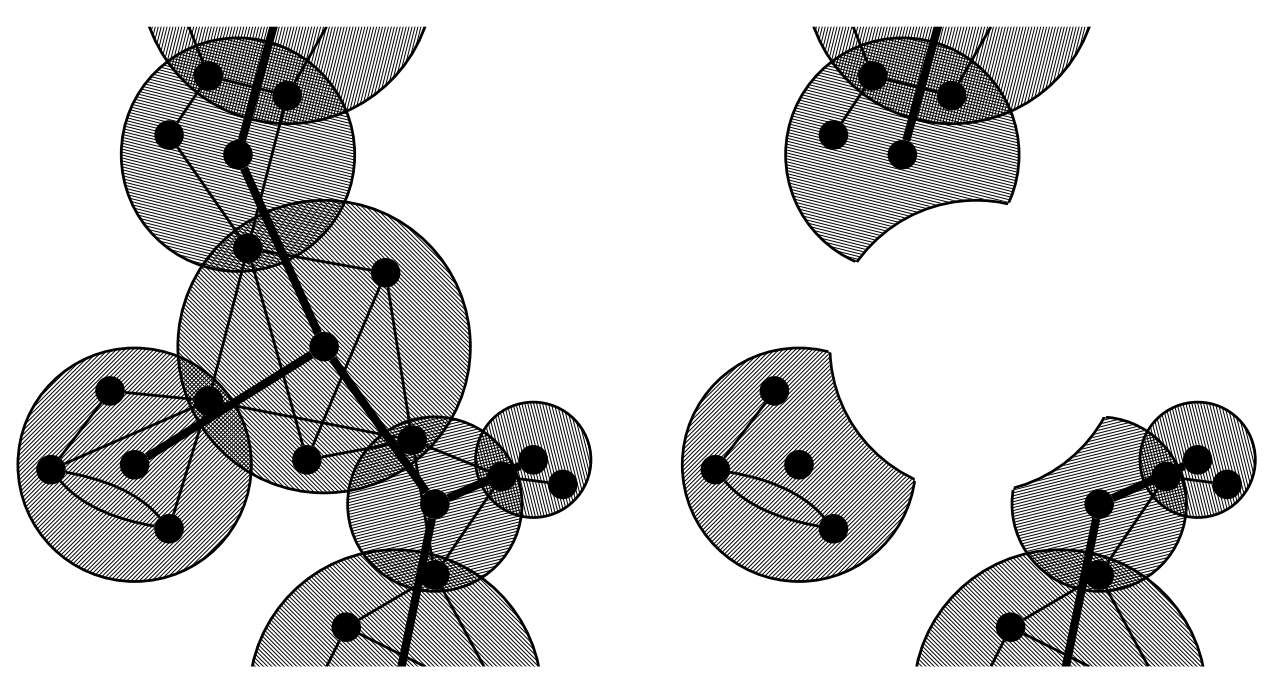

Figure 6.2: Deleting a (vertex) bag from a tree-decomposition of a graph separates the decomposition.

The key difference in the configuration context is use of contraction instead of deletion. This is necessary in order to preserve the desired separation property (see Figure 6.3). Some further explanation of this possibly striking correspondence is given in Section 5.1 .

We first require a definition.

Definition 6.1.5. Let $D$ be a configuration, let $X \subseteq D$ and let $\left\{\Re_{i} \mid i \in I\right\}$ be a collection of subsets of $D$. Then $\left\{\Re_{i} \mid i \in I\right\}$ meet properly at $X$ if $\left\{\mathfrak{R}_{i}-(\mathrm{D} \cap\langle\mathrm{X}\rangle) \mid \boldsymbol{i} \in \mathrm{I}\right\}$ are pairwise disjoint unions of $X$-bridges of $\mathrm{D} .^{2}$

Proposition 6.1.6. Let $\sigma: \mathrm{V}(\mathrm{T}) \rightarrow \mathcal{S}(\mathrm{U})$, where $\mathrm{T}$ is a tree and $\mathrm{U}$ is a vector space. Then $(\mathrm{T}, \sigma)$ satisfies Interpolation if and only if it satisfies:

${ }^{2}$ Bridges are discussed in Section 5.1. 


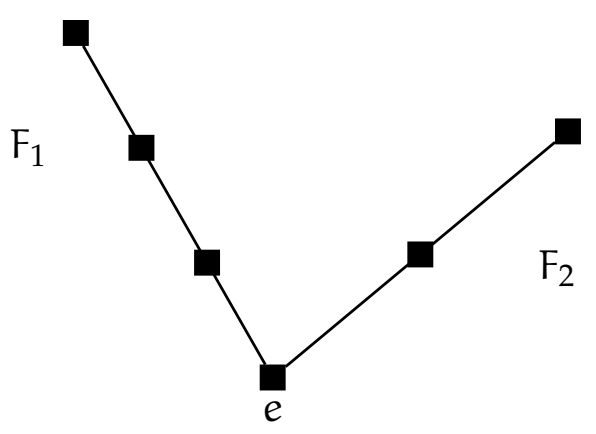

Figure 6.3: A geometric representation of a configuration in which contracting e separates $F_{1}$ and $F_{2}$, while deleting $e$ fails to do so.

(TD2') (Proper Meeting) For every $t \in V(T)$, the subspaces $\left\{S_{i} \mid i \in I\right\}$ displayed by $\mathrm{t}$ meet properly at $\sigma(\mathrm{t})$ in $\sigma(\mathrm{t}) \cup \bigcup_{i \in \mathrm{I}} S_{i}$.

Proof. Suppose that $(T, \sigma)$ satisfies Interpolation. Let $t \in V(T)$, let $\left\{S_{i} \mid i \in\right.$ I\} be the subspaces displayed by $t$ and let $j \in I$. Then

$$
\begin{aligned}
\sigma(t) \subseteq & \left\langle\left(S_{j}-\sigma(t)\right) \sqcup \sigma(t)\right\rangle \\
& \cap\left\langle\left(\sigma(t) \cup \bigcup_{i \in I} S_{i}\right)-\left(S_{j}-\sigma(t)\right)\right\rangle \\
& \subseteq\left\langle\sigma(t) \cup \bigcup_{i \neq j} S_{i}\right\rangle \cap\left(\sigma(t)+S_{j}\right) \\
= & \sigma(t)+\left(S_{j} \cap\left\langle\sigma(t) \cup \bigcup_{i \neq j} S_{i}\right\rangle\right) \\
= & \sigma(t)
\end{aligned}
$$$$
\text { by the Modular Law }
$$$$
\text { by Interpolation. }
$$

It follows from Proposition 5.1.12 that $S_{j}-\sigma(t)$ is a union of $\sigma(t)$-bridges of $\sigma(t) \cup \bigcup_{i \in I} S_{i}$. Hence Proper Meeting holds.

Now suppose that Proper Meeting holds. Let $t \in e \in E(T)$ and let $S_{1}$ and $S_{2}$ be the subspaces displayed by e. Without loss of generality, $S_{1}$ is a subspace displayed by $t$. Let $\left\{S^{i} \mid i \in I\right\}$ be the other subspaces displayed 
by t. It follows from Proper Meeting that $\left\{S^{i}-\sigma(t) \mid i \in I\right\} \sqcup\left\{S_{1}-\sigma(t)\right\}$ are pairwise disjoint unions of $\sigma(t)$-bridges of $D:=\sigma(t) \cup S_{1} \cup \bigcup_{i \in I} S^{i}$. So by Proposition 5.1.12,

$$
\begin{aligned}
\sigma(t) & =\left\langle\left(S_{1}-\sigma(t)\right) \sqcup \sigma(t)\right\rangle \cap\left\langle D-\left(S_{1}-\sigma(t)\right)\right\rangle \\
& \supseteq S_{1} \cap\left\langle\sigma(t) \cup \bigcup_{i \in I} S^{i}\right\rangle \\
& =S_{1} \cap S_{2} .
\end{aligned}
$$

It follows by symmetry that $S_{1} \cap S_{2} \subseteq \sigma(e)$. Hence Interpolation holds.

Of course, it is also possible to examine the effect of contracting an edge bag. This leads to the following separation result. Its well-known analogue for tree-decompositions of graphs is given on p. 320 of [Die06].

Proposition 6.1.7. Let $(\mathrm{T}, \sigma)$ be a tree-decomposition of a configuration, let $\mathrm{V}=\sum_{\mathrm{t} \in \mathrm{V}(\mathrm{T})} \sigma(\mathrm{t})$ and let $\mathrm{e} \in \mathrm{E}(\mathrm{T})$. Write $\mathrm{V}=\sigma(e) \oplus \mathrm{U}_{1}=\sigma(e) \oplus \mathrm{U}_{2}$ and let $\pi_{i}$ be the projection of $\mathrm{V}$ onto $\mathrm{U}_{i}$ along $\sigma(e)$ for each $i=1,2$. Then if $S_{1}$ and $S_{2}$ are the subspaces displayed by e,

$$
\pi_{1}\left[S_{1}\right] \cap \pi_{2}\left[S_{2}\right]=\{\boldsymbol{0}\}
$$

Proof. Let $x \in \pi_{1}\left[S_{1}\right] \cap \pi_{2}\left[S_{2}\right]$. Then for each $i$ there exist $s_{i} \in S_{i}$ and $s_{i}^{e} \in \sigma(e)$ such that $s_{i}=s_{i}^{e}+x$. Consequently $s_{1}=s_{2}+\left(s_{1}^{e}-s_{2}^{e}\right) \in S_{2}$, and so $s_{1} \in \sigma(e)$ by Interpolation. As $\pi_{1}\left(s_{1}\right)=x$, it follows that $x=0$.

The freedom to use any contractions by $\sigma(e)$ in the last proposition reflects its fundamentally matroidal character.

The next proposition provides a means of obtaining a tree-decomposition of a minor from a tree-decomposition of its ambient configuration. More prosaically, it also allows us to restrict our attention to the subspace spanned by a configuration. 
Proposition 6.1.8. Let $\mathrm{D}$ be a configuration, let $\mathrm{X}, \mathrm{Y} \subseteq \mathrm{D}$ be disjoint and let $(\mathrm{T}, \sigma)$ be a tree-decomposition of $\mathrm{D}$. Let $\mathrm{V}=\langle\mathrm{D}-\mathrm{X}\rangle$, let $\mathrm{W}=\langle\mathrm{Y}\rangle$ and write $\mathrm{V}=\mathrm{W} \oplus \mathrm{U}$. Let $\pi$ be the projection of $\mathrm{V}$ onto $\mathrm{U}$ along $\mathrm{W}$ and define $\sigma^{\prime}: \mathrm{V}(\mathrm{T}) \rightarrow \mathcal{S}(\mathrm{U}) b y$

$$
\sigma^{\prime}(\mathrm{t})=\pi[\mathrm{V} \cap \sigma(\mathrm{t})]
$$

for every $\mathrm{t} \in \mathrm{V}(\mathrm{T})$. Then $\left(\mathrm{T}, \mathrm{\sigma}^{\prime}\right)$ is a tree-decomposition of $\mathrm{D} \backslash \mathrm{X} / \mathrm{Y}$ having $\sigma^{\prime}(e)=\pi[V \cap \sigma(e)]$ for every $e \in E(T)$ and $w^{\prime}(x) \leqslant w(x)$ for every $x \in$ $\mathrm{V}(\mathrm{T}) \sqcup \mathrm{E}(\mathrm{T})$. In particular, the width of $\left(\mathrm{T}, \mathrm{\sigma}^{\prime}\right)$ is at most the width of $(\mathrm{T}, \sigma)$.

Proof. Clearly $\pi$ determines a contraction of $\mathrm{D} \backslash \mathrm{X}$ by $\mathrm{Y}$. It follows from (TD1) for $(T, \sigma)$ that $D-X \subseteq V \cap \bigcup_{t \in V(T)} \sigma(t)$, so $\pi[D-X] \subseteq \bigcup_{t \in V(T)} \sigma^{\prime}(t)$. Thus (TD1) holds for $\left(T, \sigma^{\prime}\right)$, so we verify Interpolation.

Let $e \in E(T)$, let $Z_{1}$ and $Z_{2}$ be the sets displayed by $e$ in $(T, \sigma)$ and let $S_{1}^{\prime}$ and $S_{2}^{\prime}$ be the corresponding subspaces displayed by $e$ in $\left(T, \sigma^{\prime}\right)$. Write $W=W_{1}+W_{2}$, where $W_{i} \leqslant\left\langle V \cap Z_{i}\right\rangle$ for each $i$. Then

$$
\begin{array}{rlrl}
\mathrm{S}_{1}^{\prime} \cap \mathrm{S}_{2}^{\prime} & =\left\langle\pi\left[\mathrm{V} \cap \mathrm{Z}_{1}\right]\right\rangle \cap\left\langle\pi\left[\mathrm{V} \cap \mathrm{Z}_{2}\right]\right\rangle & & \\
& =\pi\left[\left\langle\mathrm{V} \cap \mathrm{Z}_{1}\right\rangle\right] \cap \pi\left[\left\langle\mathrm{V} \cap \mathrm{Z}_{2}\right\rangle\right] & & \text { as } \pi \text { is linear } \\
& =\mathrm{U} \cap\left(\mathrm{W}+\left\langle\mathrm{V} \cap \mathrm{Z}_{1}\right\rangle\right) \cap\left(\mathrm{W}+\left\langle\mathrm{V} \cap \mathrm{Z}_{2}\right\rangle\right) & & \text { by Prop. 2.2.5.(4) } \\
& =\mathrm{U} \cap\left(\mathrm{W}+\left(\left(\mathrm{W}+\left\langle\mathrm{V} \cap \mathrm{Z}_{1}\right\rangle\right) \cap\left\langle\mathrm{V} \cap \mathrm{Z}_{2}\right\rangle\right)\right) & & \text { by the Modular Law } \\
& =\mathrm{U} \cap\left(\mathrm{W}+\left(\left(\mathrm{W}_{2}+\left\langle\mathrm{V} \cap \mathrm{Z}_{1}\right\rangle\right) \cap\left\langle\mathrm{V} \cap \mathrm{Z}_{2}\right\rangle\right)\right) & & \\
& =\mathrm{U} \cap\left(\mathrm{W}+\mathrm{W}_{2}+\left(\left\langle\mathrm{V} \cap \mathrm{Z}_{1}\right\rangle \cap\left\langle\mathrm{V} \cap \mathrm{Z}_{2}\right\rangle\right)\right) & & \text { by the Modular Law } \\
& \subseteq \mathrm{U} \cap(\mathrm{W}+(\mathrm{V} \cap \sigma(e))) & & \text { by Interpolation } \\
& =\pi[\mathrm{V} \cap \sigma(e)] & & \text { by Prop. 2.2.5.(4) } \\
& \subseteq \sigma^{\prime}(e) . &
\end{array}
$$

Hence Interpolation holds for $\left(\mathrm{T}, \sigma^{\prime}\right)$. In particular, $\sigma^{\prime}(e)=\pi[\mathrm{V} \cap \sigma(e)]$ for every $e \in E(T)$. The remaining observations are then clear.

The next corollary is an immediate consequence of Proposition 6.1.8. 
Corollary 6.1.9. Let $\mathrm{D}$ be a configuration and let $\mathrm{D}^{\prime} \preccurlyeq \mathrm{D}$. Then $\operatorname{tw}\left(\mathrm{D}^{\prime}\right) \leqslant$ $\operatorname{tw}(D) \leqslant \operatorname{dim}(D)$.

One property that may be desirable of a tree-decomposition is the absence of redundant bags. This idea leads to the following definition.

Definition 6.1.10. A tree-decomposition $(T, \sigma)$ is irredundant if $\sigma(t) \nsubseteq$ $\sigma\left(t^{\prime}\right)$ whenever $t, t^{\prime} \in V\left(T^{\prime}\right)$ are distinct.

The idea of irredundancy appears elsewhere in different contexts, sometimes under a different name. For example, Adler calls irredundant tree-decompositions of graphs "small" (see p. 9 of [Ad106]), while Heule and Kullmann define irredundancy more generally for forest decompositions of finite hypergraphs (see pp.15-16 of [HK06]).

The next proposition says that finite tree-decompositions can always be made irredundant. This is later extended to tree-decompositions having finite width in Proposition 6.4.12.

Proposition 6.1.11. Whenever a configuration has a finite tree-decomposition $(\mathrm{T}, \sigma)$ of width $w$, it has a finite irredundant tree-decomposition $\left(\mathrm{T}^{\prime}, \sigma^{\prime}\right)$ of width $w$ for which $\operatorname{im}\left(\sigma^{\prime}\right) \subseteq \operatorname{im}(\sigma)$.

Proof. Let D be a configuration having a finite tree-decomposition $(\mathrm{T}, \sigma)$ of width $w$ and let $\mathcal{T}$ be the collection of all finite tree-decompositions $\left(\mathrm{T}^{\prime}, \sigma^{\prime}\right)$ of $\mathrm{D}$ having width $w$ and $\operatorname{im}\left(\sigma^{\prime}\right) \subseteq \operatorname{im}(\sigma)$. Clearly $\mathcal{T}$ is non-empty. For every $\left(T^{\prime}, \sigma^{\prime}\right) \in \mathcal{T}$, let

$$
\mathrm{J}\left(\mathrm{T}^{\prime}, \sigma^{\prime}\right)=\sum_{\text {Distinct } \mathrm{t}, \mathrm{t}^{\prime} \in \mathrm{V}\left(\mathrm{T}^{\prime}\right)} \mathbf{1}\left(\sigma(\mathrm{t}), \sigma\left(\mathrm{t}^{\prime}\right)\right)
$$

where

$$
\mathbf{I}(X, Y):= \begin{cases}1 & \text { if } X \subseteq Y, \text { and } \\ 0 & \text { otherwise. }\end{cases}
$$

Then $J$ counts the number of times that one bag of $\left(T^{\prime}, \sigma^{\prime}\right)$ is contained in another. Clearly $\mathrm{J}\left(\mathrm{T}^{\prime}, \sigma^{\prime}\right) \in \omega$. 
Recall that whenever $T$ is a tree and $t, t^{\prime} \in V(T)$, the unique path in $T$ linking $t$ and $t^{\prime}$ is denoted $t T t^{\prime}$.

Let $\left(T^{\prime}, \sigma^{\prime}\right) \in \mathcal{T}$ have $J\left(T^{\prime}, \sigma^{\prime}\right)$ minimal. Suppose that $J\left(T^{\prime}, \sigma^{\prime}\right)>0$. Then there exist distinct $t, t^{\prime} \in V(T)$ such that $\sigma(t) \subseteq \sigma\left(t^{\prime}\right)$. Write $N(t)=$ $\left\{t_{1}, \ldots, t_{n}\right\}$, where $t_{1}$ is the neighbour of $t$ in $t T t^{\prime}$. Let

$$
T^{\prime \prime}=\left(V\left(T^{\prime}\right)-\{t\},\left(E\left(T^{\prime}\right)-\left\{t_{1}, \ldots, t t_{n}\right\}\right) \sqcup\left\{t_{1} t_{2}, \ldots, t_{1} t_{n}\right\}\right)
$$

as shown in Figure 6.4 and set $\sigma^{\prime \prime}=\left.\sigma\right|_{V\left(T^{\prime \prime}\right)}$.
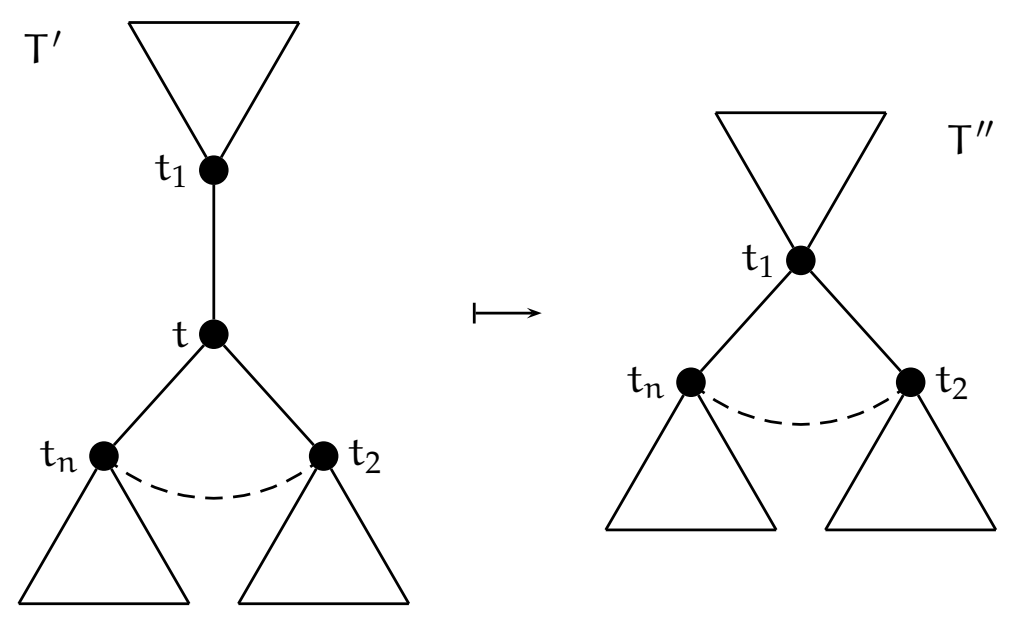

Figure 6.4: Surgery on the tree-decomposition. The redundant vertex $t$ is replaced by edges incident on its neighbour $t_{1}$.

Clearly $\left(\mathrm{T}^{\prime \prime}, \sigma^{\prime \prime}\right) \in \mathcal{T}$. Moreover, $\mathrm{J}\left(\mathrm{T}^{\prime \prime}, \sigma^{\prime \prime}\right)<\mathrm{J}\left(\mathrm{T}^{\prime}, \sigma^{\prime}\right)$, contradicting the minimality of the latter. Hence $J\left(T^{\prime}, \sigma^{\prime}\right)=0$, and so $\left(T^{\prime}, \sigma^{\prime}\right)$ is irredundant.

The next proposition shows that it is possible to induce a tree-decomposition of a configuration whenever its points label the vertices of a tree. 
Proposition 6.1.12. Let $\mathrm{T}$ be a tree, let $\mathrm{D}$ be a configuration and let $\tau: \mathrm{D} \rightarrow$ $\mathrm{V}(\mathrm{T})$. Define $\sigma: \mathrm{E}(\mathrm{T}) \rightarrow \mathcal{S}(\langle\mathrm{D}\rangle)$ by

$$
\sigma(e)=\left\langle\tau^{-1} V\left(T_{1}\right)\right\rangle \cap\left\langle\tau^{-1} V\left(T_{2}\right)\right\rangle
$$

for every $e \in E(T)$, where $T_{1}$ and $T_{2}$ are the connected components of $\mathrm{T}-\{e\}$. Extend $\sigma$ to $\mathrm{V}(\mathrm{T}) \sqcup \mathrm{E}(\mathrm{T})$ by setting

$$
\sigma(t)=\left\langle\tau^{-1}\{t\}\right\rangle+\sum_{t^{\prime} \in N(t)} \sigma\left(t t^{\prime}\right)
$$

for every $\mathrm{t} \in \mathrm{V}(\mathrm{T})$. Then $\left(\mathrm{T},\left.\mathrm{\sigma}\right|_{\mathrm{V}(\mathrm{T})}\right)$ is a tree-decomposition of $\mathrm{D}$.

Proof. The statement (TD1) clearly holds, so we consider Interpolation. Let $e \in E(T)$, let $T_{1}$ and $T_{2}$ be the connected components of $T-\{e\}$ and let $S_{1}$ and $S_{2}$ be the corresponding subspaces displayed by e. Then as $\tau^{-1} \mathrm{~V}\left(\mathrm{~T}_{i}\right) \subseteq \bigcup_{\mathrm{t} \in \mathrm{V}\left(\mathrm{T}_{i}\right)} \sigma(\mathrm{t})$ for each $i$, clearly

$$
\begin{aligned}
\left\langle\tau^{-1} V\left(T_{1}\right)\right\rangle \cap\left\langle\tau^{-1} V\left(T_{2}\right)\right\rangle & \subseteq\left\langle\bigcup_{t \in V\left(T_{1}\right)} \sigma(t)\right\rangle \cap\left\langle\bigcup_{t^{\prime} \in V\left(T_{2}\right)} \sigma\left(t^{\prime}\right)\right\rangle \\
\sigma(e) & \subseteq S_{1} \cap S_{2} .
\end{aligned}
$$

If $t \in V\left(T_{i}\right)$, then $\sigma\left(t t^{\prime}\right) \subseteq\left\langle\tau^{-1} V\left(T_{i}\right)\right\rangle$ for every $t^{\prime} \in N(t)$, so $\sigma(t) \subseteq$ $\left\langle\tau^{-1} \mathrm{~V}\left(\mathrm{~T}_{\mathrm{i}}\right)\right\rangle$. Consequently

$$
\begin{gathered}
\left\langle\bigcup_{t \in V\left(T_{1}\right)} \sigma(t)\right\rangle \cap\left\langle\bigcup_{t^{\prime} \in \mathrm{V}\left(\mathrm{T}_{2}\right)} \sigma\left(\mathrm{t}^{\prime}\right)\right\rangle \subseteq\left\langle\tau^{-1} \mathrm{~V}\left(\mathrm{~T}_{1}\right)\right\rangle \cap\left\langle\tau^{-1} \mathrm{~V}\left(\mathrm{~T}_{2}\right)\right\rangle \\
\mathrm{S}_{1} \cap \mathrm{S}_{2} \subseteq \sigma(e) .
\end{gathered}
$$

Hence $S_{1} \cap S_{2}=\sigma(e)$, and so Interpolation holds.

This result suggests the following definition.

Definition 6.1.13. Let $T$ be a tree, let $D$ be a configuration and let $\tau: D \rightarrow$ 
$\mathrm{V}(\mathrm{T})$. Then the tree-decomposition of $\mathrm{D}$ induced by $\tau$ is the tree-decomposition of $\mathrm{D}$ derived from $\tau$ via Proposition 6.1.12. Moreover, a tree-decomposition $(T, \sigma)$ of $D$ is leaf-induced if there exists $\tau: D \rightarrow L(T)$ such that $(T, \sigma)$ is the tree-decomposition of $D$ induced by $\tau$.

The results below investigate some properties of induced tree-decompositions. Several of these are used in Section 6.2 to obtain a tree-decomposition of a finite configuration that "looks like" a branch-decomposition. This permits the easy adaptation to tree-decompositions of an argument for the existence of linked branch-decompositions.

Recall that a tree is cubic if each of its non-leaf vertices has degree 3.

Formally, a branch-decomposition of a matroid $M$ is a pair $(T, \tau)$, where $T$ is a finite cubic tree and $\tau: E(M) \rightarrow L(T)$. Each edge $e \in E(T)$ is associated with a partition of $E(M)$, namely $\left\{\tau^{-1} V\left(T_{1}\right), \tau^{-1} V\left(T_{2}\right)\right\}$, where $T_{1}$ and $T_{2}$ are the connected components of $T-\{e\}$. The width of $e$, denoted $w(e)$, is $\lambda_{M}\left(\tau^{-1} V\left(T_{1}\right)\right)+1=\lambda_{M}\left(\tau^{-1} V\left(T_{2}\right)\right)+1$. The width of $(T, \tau)$ is

$$
\max \{w(e) \mid e \in \mathrm{E}(\mathrm{T})\}
$$

and the branch-width of $M$ is the maximum of the widths of its branchdecompositions.

The next result provides the first step towards a "branch-decomposition-like" tree-decomposition by allowing the parallel classes of a configuration to be "injected into" the leaves of a tree-decomposition.

Proposition 6.1.14. Every tree-decomposition $(\mathrm{T}, \sigma)$ of a configuration $\mathrm{D}$ may be extended to a tree-decomposition $\left(\mathrm{T}^{\prime}, \sigma^{\prime}\right)$ of $\mathrm{D}$ of the same width for which

$$
\{\langle\mathrm{d}\rangle \mid \mathrm{d} \in \mathrm{D}\} \subseteq\left\{\sigma^{\prime}(\mathrm{t}) \mid \mathrm{t} \in \mathrm{L}\left(\mathrm{T}^{\prime}\right)\right\} .
$$

Moreover, if $(\mathrm{T}, \sigma)$ and $\widetilde{\mathrm{D}}$ are finite, then so is $\left(\mathrm{T}^{\prime}, \sigma^{\prime}\right)$.

Proof. Let $I=\{\langle d\rangle \mid d \in D\}$ and let $f$ be a choice function for $\{\{t \in V(T) \mid$ 
$i \subseteq \sigma(t)\} \mid i \in I\}$. Then set

$$
\mathrm{T}^{\prime}=\mathrm{T} \cup(\psi[\mathrm{I}],\{\{\mathrm{f}(\mathrm{i}), \psi(i)\} \mid i \in \mathrm{I}\})
$$

where $\psi$ is any bijection having domain I for which $\mathrm{V}(\mathrm{T}) \cap \psi[\mathrm{I}]$ is empty. Define $\sigma^{\prime}$ by $\left.\sigma^{\prime}\right|_{V(T)}=\sigma$ and $\sigma^{\prime} \circ \psi=\mathrm{id}_{\mathrm{I}}$. Then (TD1) clearly holds and Interpolation follows from $\left(\sigma^{\prime} \circ \psi\right)(i)=i \subseteq(\sigma \circ f)(i)$ for every $i \in$ I. Consequently $\left(T^{\prime}, \sigma^{\prime}\right)$ is a tree-decomposition of D. The remaining observations are then clear.

The next result says that a finite tree-decomposition can always be altered so that its underlying tree has maximum degree three (in this case the tree is said to be subcubic).

Proposition 6.1.15. Whenever a configuration has a finite tree-decomposition $(T, \sigma)$ of width $w$, it has a finite tree-decomposition $\left(T^{\prime}, \sigma^{\prime}\right)$ of width $w$ such that

(1) $\Delta\left(\mathrm{T}^{\prime}\right) \leqslant 3$, and

(2) $\left.\sigma\right|_{\mathrm{L}(\mathrm{T})}=\sigma^{\prime} \circ \psi$ for some bijection $\psi: \mathrm{L}(\mathrm{T}) \rightarrow \mathrm{L}\left(\mathrm{T}^{\prime}\right)$.

Proof. Let D be a configuration having a finite tree-decomposition $(T, \sigma)$ of width $w$. Let $\mathcal{T}$ be the collection of all finite tree-decompositions $\left(T^{\prime}, \sigma^{\prime}\right)$ of $\mathrm{D}$ having width $w$ for which (2) holds. Clearly $\mathcal{T}$ is non-empty. For every finite tree $\mathrm{T}^{\prime}$, let

$$
J\left(T^{\prime}\right)=\sum_{t \in V\left(T^{\prime}\right)} \max \{0, d(t)-3\}
$$

Then $J$ counts the number of neighbours beyond 3 across the vertices of $\mathrm{T}^{\prime}$. Clearly $\mathrm{J}\left(\mathrm{T}^{\prime}\right) \in \omega$.

Let $\left(T^{\prime}, \sigma^{\prime}\right) \in \mathcal{T}$ have $J\left(T^{\prime}\right)$ minimal. Suppose that $J\left(T^{\prime}\right)>0$. Then there exists $t \in V\left(T^{\prime}\right)$ having $d(t)>3$. Write $N(t)=\left\{t_{1}, \ldots, t_{n}\right\}$ and let $T^{\prime \prime}$ 
be the tree having

$$
\begin{aligned}
V\left(T^{\prime \prime}\right)= & \left(V\left(T^{\prime}\right)-\{t\}\right) \sqcup \operatorname{im}(\psi), \text { and } \\
E\left(T^{\prime \prime}\right)= & \left(E\left(T^{\prime}\right)-\left\{t_{1}, \ldots, t t_{n}\right\}\right) \\
& \sqcup\left\{\operatorname{im}(\psi),\left\{\psi(1), t_{1}\right\},\left\{\psi(1), t_{2}\right\},\left\{\psi(2), t_{3}\right\}, \ldots,\left\{\psi(2), t_{n}\right\}\right\}
\end{aligned}
$$

as shown in Figure 6.5, where $\psi$ is any bijection having domain $\{1,2\}$ for which $V\left(T^{\prime}\right) \cap \operatorname{im}(\psi)$ is empty.
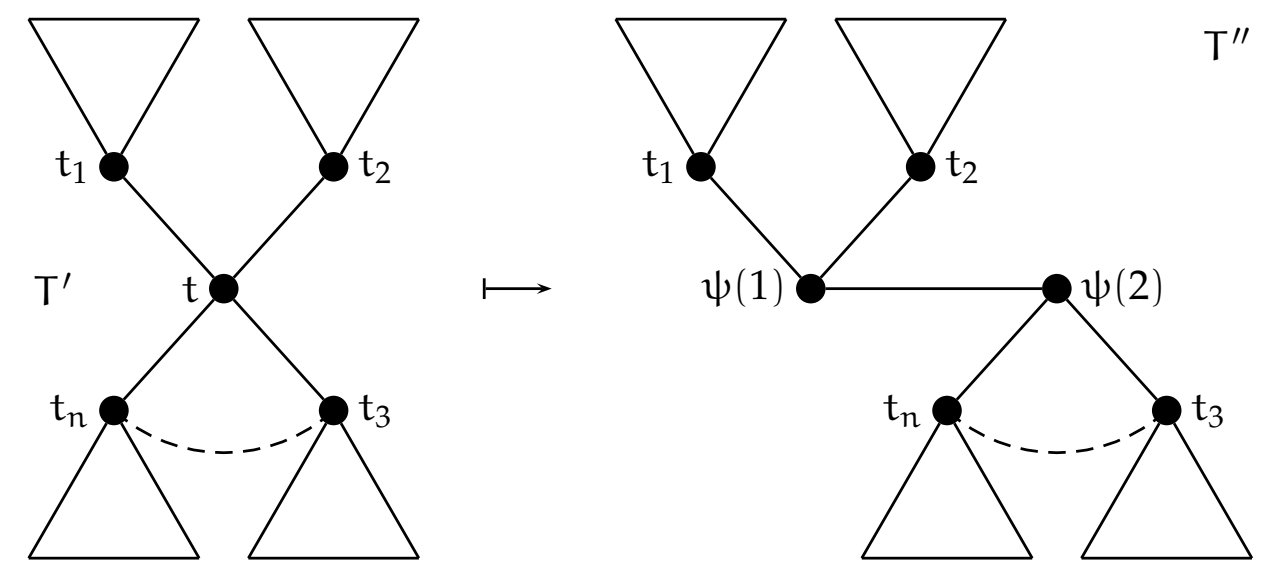

Figure 6.5: Surgery on the tree decomposition. The high-degree vertex $t$ is replaced by an edge $\operatorname{im}(\psi)$ such that $d(\psi(1))=3$.

Define $\sigma^{\prime \prime}$ by $\left.\sigma^{\prime \prime}\right|_{\mathrm{V}\left(\mathrm{T}^{\prime}\right)-\{\mathrm{t}\}}=\left.\sigma^{\prime}\right|_{\mathrm{V}\left(\mathrm{T}^{\prime}\right)-\{\mathrm{t}\}}$ and $\left(\sigma^{\prime \prime} \circ \psi\right)(1)=\left(\sigma^{\prime \prime} \circ \psi\right)(2)=$ $\sigma^{\prime}(t)$. Clearly $\left(T^{\prime \prime}, \sigma^{\prime \prime}\right) \in \mathcal{T}$. Moreover, $J\left(T^{\prime \prime}\right)<J\left(T^{\prime}\right)$, contradicting the minimality of the latter. Hence $\mathrm{J}\left(\mathrm{T}^{\prime}\right)=0$, and so $\Delta\left(\mathrm{T}^{\prime}\right) \leqslant 3$.

Given a tree-decomposition of a configuration, the following result establishes the existence of "smaller" induced tree-decompositions of subconfigurations.

Proposition 6.1.16. Let $(\mathrm{T}, \sigma)$ be a tree-decomposition of a configuration $\mathrm{D}$ and let $\mathrm{D}^{\prime} \leqslant \mathrm{D}$. Then whenever $\tau: \widetilde{\mathrm{D}^{\prime}} \rightarrow \mathrm{V}(\mathrm{T})$ has $\mathrm{d} \in(\sigma \circ \tau)(\mathrm{d})$ for every $\mathrm{d} \in \widetilde{\mathrm{D}^{\prime}}$ 
and

$$
T^{\prime}:=\bigcup_{d \in \widetilde{D^{\prime}}} \tau(d) T \tau\left(d^{\prime}\right)
$$

for some fixed $\mathrm{d}^{\prime} \in \widetilde{\mathrm{D}^{\prime}}, \tau$ induces a tree-decomposition $\left(\mathrm{T}^{\prime}, \sigma^{\prime}\right)$ of $\mathrm{D}^{\prime}$ such that

(1) $\sigma^{\prime}(t) \leqslant \sigma(t)$ for every $t \in V\left(T^{\prime}\right)$, and

(2) $\sigma^{\prime}(e) \leqslant \sigma(e)$ for every $e \in E\left(T^{\prime}\right)$.

Moreover, the width of $\left(\mathrm{T}^{\prime}, \sigma^{\prime}\right)$ is at most the width of $(\mathrm{T}, \sigma)$, and $\left(\mathrm{T}^{\prime}, \sigma^{\prime}\right)$ is finite if $\widetilde{\mathrm{D}^{\prime}}$ is finite.

Proof. The definition of $\mathrm{T}^{\prime}$ ensures that $\tau$ is into $\mathrm{V}\left(\mathrm{T}^{\prime}\right)$, so $\tau$ induces a tree-decomposition $\left(\mathrm{T}^{\prime}, \sigma^{\prime}\right)$ of $\widetilde{\mathrm{D}^{\prime}}$, which is a tree-decomposition of $\mathrm{D}^{\prime}$ by Corollary 6.1.4. We demonstrate (2) and then use this to establish (1).

Let $e \in E\left(T^{\prime}\right)$, let $T_{1}^{\prime}$ and $T_{2}^{\prime}$ are the connected components of $T^{\prime}-\{e\}$, and let $T_{1}$ and $T_{2}$ be the corresponding connected components of $T-\{e\}$. Then

$$
\begin{aligned}
\sigma^{\prime}(e) & =\left\langle\tau^{-1} V\left(T_{1}^{\prime}\right)\right\rangle \cap\left\langle\tau^{-1} V\left(T_{2}^{\prime}\right)\right\rangle \\
& \leqslant\left\langle\bigcup_{t \in V\left(T_{1}\right)} \sigma(t)\right\rangle \cap\left\langle\bigcup_{t^{\prime} \in V\left(T_{2}\right)} \sigma\left(t^{\prime}\right)\right\rangle \\
& =\sigma(e) .
\end{aligned}
$$

Thus (2) holds. Now let $t \in V\left(T^{\prime}\right)$. Then

$$
\begin{aligned}
\sigma^{\prime}(t) & =\left\langle\tau^{-1}\{t\}\right\rangle+\sum_{t^{\prime} \in N_{T^{\prime}}(t)} \sigma^{\prime}\left(t t^{\prime}\right) \\
& \leqslant\left\langle\tau^{-1}\{t\}\right\rangle+\sum_{t^{\prime} \in N_{T}(t)} \sigma\left(t t^{\prime}\right) \\
& \leqslant \sigma(t) .
\end{aligned}
$$

Hence (1) holds. The remaining observations are then clear. 
Taking $\mathrm{D}^{\prime}=\mathrm{D}$ in Proposition 6.1.16 gives the next corollary, which shows that we may often assume that a tree-decomposition is induced without loss of generality.

Corollary 6.1.17. Let $(T, \sigma)$ be a tree-decomposition of a configuration $\mathrm{D}$. Then whenever $\tau: \widetilde{\mathrm{D}} \rightarrow \mathrm{V}(\mathrm{T})$ has $\mathrm{d} \in(\sigma \circ \tau)(\mathrm{d})$ for every $\mathrm{d} \in \widetilde{\mathrm{D}}$ and

$$
T^{\prime}:=\bigcup_{d \in \widetilde{D}} \tau(d) T \tau\left(d^{\prime}\right)
$$

for some fixed $\mathrm{d}^{\prime} \in \widetilde{\mathrm{D}}, \tau$ induces a tree-decomposition $\left(\mathrm{T}^{\prime}, \sigma^{\prime}\right)$ of $\mathrm{D}$ such that

(1) $\sigma^{\prime}(t) \leqslant \sigma(t)$ for every $t \in V\left(T^{\prime}\right)$, and

(2) $\sigma^{\prime}(e) \leqslant \sigma(e)$ for every $e \in \mathrm{E}\left(\mathrm{T}^{\prime}\right)$.

Moreover, the width of $\left(\mathrm{T}^{\prime}, \sigma^{\prime}\right)$ is at most the width of $(\mathrm{T}, \sigma)$, and $\left(\mathrm{T}^{\prime}, \sigma^{\prime}\right)$ is finite if $\widetilde{\mathrm{D}}$ is finite.

The next corollary is a useful consequence of Corollary 6.1.17.

Corollary 6.1.18. Every configuration having finite simplification and a treedecomposition of width $w$ has a finite tree-decomposition of width at most $w$.

\subsection{Linked Decompositions: The Finite Case}

We open this section with a simple consequence of Interpolation. Say that a path is non-trivial if it contains at least one edge. Then we have the following proposition.

Proposition 6.2.1. Let $(T, \sigma)$ be a tree-decomposition of a configuration $\mathcal{D}=$ $(\mathrm{D}, \mathrm{E}, \ell)$ and let $\mathrm{P}$ be a non-trivial path in $\mathrm{T}$ linking $\mathrm{t}_{1}$ and $\mathrm{t}_{2}$. Then

$$
\kappa\left(\ell^{-1} X_{1}, \ell^{-1} X_{2}\right) \leqslant \min \{w(e) \mid e \in E(P)\}
$$


where $X_{i}$ is the set associated with the component of $T-E(P)$ containing $t_{i}$ for each $i$.

Proof. Let $\mathrm{D}$ be the point set of $\mathcal{D}$. Then simply observe that the set of subspaces displayed by each $e \in E(P)$ induces a split of $D$, one element of which contains $D \cap X_{1}$ and the other of which contains $D \cap X_{2}$.

Recall that whenever $T$ is a tree and $t_{1}, t_{2} \in V(T)$, the unique path in $T$ linking $t_{1}$ and $t_{2}$ is denoted $t_{1} T t_{2}$.

It is possible that (6.2.1) may be strict somewhere (or even everywhere) in a tree-decomposition. However, if it holds with equality everywhere in a tree-decomposition, then the decomposition can be used to certify minimum levels of connectivity between different "branches" of its configuration. This important possibility that motivates the following definition.

Definition 6.2.2. Let $(T, \sigma)$ be a tree-decomposition of a configuration $\mathcal{D}=$ $(D, E, \ell)$ and let $e_{1}=t_{1} t_{1}^{\prime}, e_{2}=t_{2}^{\prime} t_{2} \in E(P)$, where $P=t_{1} T t_{2}$. Then $e_{1}$ and $e_{2}$ are $\mathcal{D}$-linked if

$$
\kappa\left(\ell^{-1} X_{1}, \ell^{-1} X_{2}\right)=\min \{w(e) \mid e \in E(P)\}
$$

where $X_{i}$ is the set associated with the component of $T-E(P)$ containing $t_{i}$ for each $i$. A tree-decomposition $(T, \sigma)$ is $\mathcal{D}$-linked or a linked tree-decomposition of $\mathcal{D}$ if every pair of edges of $T$ is $\mathcal{D}$-linked. The collection of all linked tree-decompositions of a configuration $\mathcal{D}$ is denoted by $\mathcal{L} \mathcal{T} \mathcal{D}(\mathcal{D})$.

This definition differs in three ways from that given for graphs on (for example) p. 87 of [KT91], namely

- it uses the connectivity function $\mathrm{k}$ instead of vertex-disjoint paths,

- it uses the associated set $X_{i}$ instead of the bag $\sigma\left(t_{i}\right)$, and

- it involves a minimum over edge bags instead of a minimum over vertex bags. 
The use of $k$ is unsurprising, as vertex-disjoint paths are a concrete realisation of connectivity in graphs. It is necessary to use the entire associated set $X_{i}$ instead of $\sigma\left(t_{i}\right)$ to allow for the possibility that $\sigma\left(t_{i}\right)$ may be entirely or partially "induced"-that is, not spanned by the points of the configuration that it contains. Finally, the use of edge bags is a convenience that eliminates the need to subdivide tree edges to work with vertex bags. It has the added advantage of making the definition more closely resemble that of branch-decompositions. This last property is exploited in the proof of Theorem 6.2.5.

The next proposition is a consequence of Corollary 6.1.4 and Proposition 4.5.2.

Proposition 6.2.3. Let $\mathcal{D}$ be a configuration with point set $\mathrm{D}$. Then $\mathcal{L} \mathcal{T} \mathcal{D}(\mathcal{D})=$ $\mathcal{L} \mathcal{T} \mathcal{D}(\mathrm{D})=\mathcal{L} \mathcal{T} \mathcal{D}(\widetilde{\mathcal{D}})$.

So it suffices in the context of linked tree-decompositions to simply view configurations as subsets of their ambient vector spaces.

Linked tree-decompositions were first introduced by Thomas for finite graphs in [Tho90], where Theorem 1.1.5 was proven. Their importance arises from their use in well-quasi-ordering proofs (see [Tho89a, RS90]): by certifying minimum levels of connectivity between the "branches" of a tree-like object, they demonstrate that certain minors can be formed. These can subsequently be used to obtain a finite certificate disproving the existence of a minimal bad sequence.

Theorem 6.2.5 below is an analogue of Theorem 1.1.5 for configurations. Its proof adapts an argument of Geelen, Gerards and Whittle given in [GGW02].

A connectivity function is a symmetric submodular function $\mathcal{P}(E) \rightarrow \omega$, where $E$ is a finite set. It is easy to define branch-decompositions for such functions. These generalise both branch-decompositions of finite graphs and branch-decompositions of matroids. Geelen, Gerards and Whittle proved the following theorem. 
Theorem 6.2.4 (Geelen, Gerards and Whittle). Every connectivity function having branch-width $w$ has a linked branch-decomposition of width $w$.

Our proof manipulates a tree-decomposition so that it "looks like" a branch-decomposition, allowing the application of Geelen, Gerards and Whittle's argument. Working primarily with edge bags in this way avoids some technicalities intrinsic to tree-decompositions, but leads to a width bound that, while good, is probably not tight.

Theorem 6.2.5. Every finite configuration $\mathrm{D}$ has a finite linked leaf-induced tree-decomposition $(\mathrm{T}, \sigma)$ of width at most $2 \mathrm{tw}(\mathrm{D})$ for which $\Delta(\mathrm{T}) \leqslant 3$.

Proof. Let $\mathcal{T}$ be the collection of all finite leaf-induced tree-decompositions $(T, \sigma)$ of $D$ for which $\Delta(T) \leqslant 3$.

(1) $\mathcal{T}$ contains a tree-decomposition of width $\mathrm{tw}(\mathrm{D})$.

$D$ has a tree-decomposition of width $\operatorname{tw}(D)$, so it has a finite treedecomposition of width $\operatorname{tw}(\mathrm{D})$ by Corollary 6.1.18. It follows from Proposition 6.1.14 that $\mathrm{D}$ has a finite tree-decomposition $(\mathrm{T}, \sigma)$ of width $\operatorname{tw}(D)$ for which $D \subseteq \bigcup_{t \in L(T)} \sigma(t)$. In light of Proposition 6.1.15, $\Delta(T) \leqslant 3$ without loss of generality. It follows from Corollary 6.1.17 that $\mathrm{D}$ has a finite leaf-induced tree-decomposition $(T, \sigma)$ of width $\operatorname{tw}(\mathrm{D})$ for which $\Delta(T) \leqslant 3$.

Let $(T, \sigma)$ be an element of $\mathcal{T}$ that is minimal with respect to the partial order $\leqslant_{E}$ defined on p. 9. Such an element exists because $\mathcal{T}$ is non-empty by (1). Suppose that $(T, \sigma)$ is induced by $\tau$.

(2) $\max \{w(e) \mid e \in E(T)\} \leqslant \operatorname{tw}(D)$.

For suppose otherwise. Let $k=\max \{w(e) \mid e \in E(T)\}$. It follows from (1) that $\mathcal{T}$ contains a tree-decomposition $\left(T^{\prime}, \sigma^{\prime}\right)$ of width $t w(D)$. Clearly $e\left(T_{\geqslant l}^{\prime}\right)=e\left(T_{\geqslant l}\right)$ and $c\left(T_{\geqslant l}^{\prime}\right)=c\left(T_{\geqslant l}\right)$ for every $l \in \mathbb{Z}_{>k}$. Moreover, $e\left(T_{\geqslant k}^{\prime}\right)<e\left(T_{\geqslant k}\right)$, so $\left(T^{\prime}, w^{\prime}\right)<_{E}(T, w)$, contradicting the minimality of $(T, \sigma)$. 
For every subgraph $G$ of $T$, let the set displayed by $G$ be $\tau^{-1} V(G)$. Then let the sets displayed by $e \in E(T)$ be the sets displayed by the connected components of $\mathrm{T}-\{e\}$.

(3) $(T, \sigma)$ has width at most $2 \mathrm{tw}(\mathrm{D})$.

Let $t \in V(T)$. If $t \in L(T)$, then $w(t)=\operatorname{dim}(\langle d\rangle)$ for some $d \in D$, so necessarily $w(t) \leqslant 2 \operatorname{tw}(D)$. If $d(t)=2$, then $\tau^{-1}\{t\}=\varnothing$, so $\sigma(t)=\sigma\left(t^{\prime}\right)$ for each $t^{\prime} \in N(t)$. But then $w(t) \leqslant 2 t w(D)$ by (2). Lastly, suppose that $d(t)=3$. Then $\sigma(t)=\sum_{i=1}^{3} \sigma\left(t t_{i}\right)$, where $N(t)=\left\{t_{1}, t_{2}, t_{3}\right\}$. Then by Proposition 4.4.6,

$$
w(t)=\sum_{i=1}^{3} \operatorname{dim}\left(\left\langle D-D_{i}\right\rangle\right)-2 \operatorname{dim}(D)
$$

where $D_{i}$ is the set displayed by the component of $T-\left\{t_{i}\right\}$ not containing $t$ for each $i$. It follows from (2) that for each $i$,

$$
\begin{aligned}
\operatorname{tw}(D) & \geqslant w\left(t_{i}\right) \\
& =\operatorname{dim}\left(\left\langle D_{i}\right\rangle \cap\left\langle D-D_{i}\right\rangle\right) \\
& =\operatorname{dim}\left(\left\langle D_{i}\right\rangle\right)+\operatorname{dim}\left(\left\langle D-D_{i}\right\rangle\right)-\operatorname{dim}(D) \\
\operatorname{dim}\left(\left\langle D_{i}\right\rangle\right)+\operatorname{dim}\left(\left\langle D-D_{i}\right\rangle\right) & \leqslant \operatorname{dim}(D)+\operatorname{tw}(D) .
\end{aligned}
$$

Thus $w(t) \leqslant 2 \operatorname{tw}(D)$ by Proposition 4.2.7. In this case, the factor of 2 allows for the possibility of non-trivial interactions between the edge bags $\sigma\left(t_{1}\right), \sigma\left(t_{2}\right)$ and $\sigma\left(t_{3}\right)$.

The remainder of our argument is the same as that given on pp. 273-275 of [GGW02].

Suppose that $(T, \sigma)$ is not D-linked. Then there exist $e_{1}, e_{2} \in E(T)$ that are not D-linked. Necessarily $e_{1} \neq e_{2}$. For each $i$, let $T_{i}$ be the component of $T-\left\{e_{i}\right\}$ not containing $e_{3-i}$, let $D_{i}$ be the set it displays and let $t_{i}$ be the endvertex of $e_{i}$ such that $e_{i} \notin \mathrm{E}\left(\mathrm{t}_{1} \mathrm{Tt}_{2}\right)$. 
Say that $\mathrm{X} \subseteq \mathrm{D}$ splits $\mathrm{Y} \subseteq \mathrm{D}$ if both $\mathrm{X} \cap \mathrm{Y}$ and $\mathrm{Y}-\mathrm{X}$ are non-empty. Note that it is possible that $X$ splits $Y$ but not vice versa. Let $A \subseteq D-D_{2}$ contain $D_{1}$ and be such that $\lambda(A)=\kappa\left(D_{1}, D_{2}\right)$ and such that $A$ splits as few sets displayed by edges of $\mathrm{T}$ as possible.

Define a new tree $\hat{T}$ as follows: for each $i$, let $T^{i}$ be a copy of the component of $T-\left\{e_{3-i}\right\}$ containing $e_{i}$. We adopt a convention of denoting the copy of $x \in V(T) \sqcup E(T)$ appearing in $T^{i}$ (if any) by $x^{i}$. Connect $T^{1}$ with $T^{2}$ by a new edge $t_{2}^{1} t_{1}^{2}$ joining $t_{2}^{1}$ to $t_{1}^{2}$ as shown in Figure 6.6. Clearly $\Delta(\hat{\top}) \leqslant 3$.

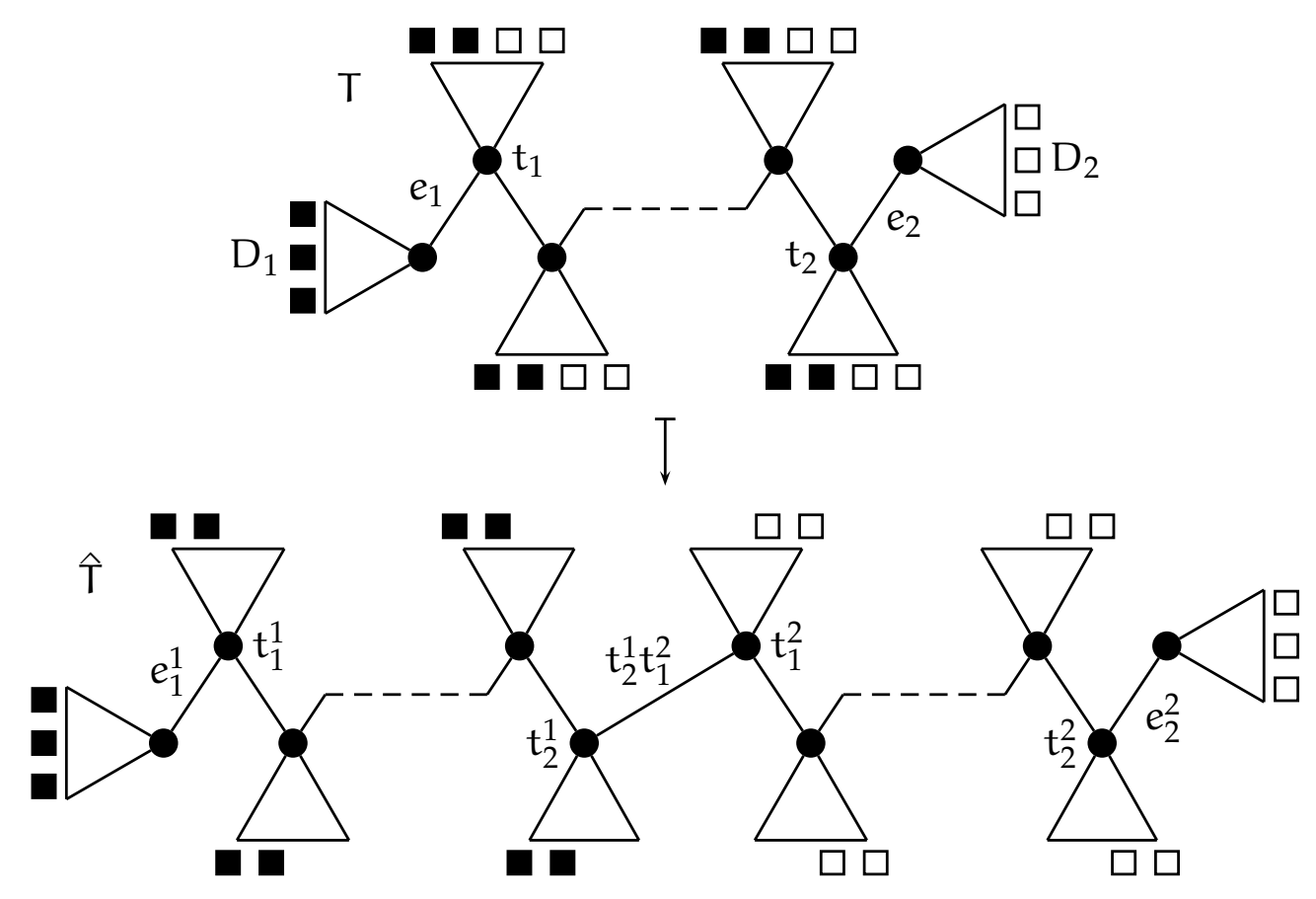

Figure 6.6: Surgery on the tree-decomposition. Black squares and white squares are intended to suggest points in $A$ and $D-A$ respectively. 
Define $\hat{\tau}: \mathrm{D} \rightarrow \mathrm{L}(\hat{\mathrm{T}})$ by

$$
\hat{\tau}(d)= \begin{cases}(\tau(d))^{1} & \text { if } d \in A, \text { and } \\ (\tau(d))^{2} & \text { otherwise }\end{cases}
$$

for every $d \in D$. Then $\hat{\tau}$ induces a tree-decomposition $(\hat{T}, \hat{\sigma}) \in \mathcal{T}$. We shall argue that $(\hat{T}, \hat{\sigma})<_{E}(T, \sigma)$, contradicting the choice of $(T, \sigma)$. To do so, we first establish the following claim.

(4) Let $e \in \mathrm{E}(\hat{\mathrm{T}})$. Then $\hat{w}\left(e^{i}\right) \leqslant w(e)$ for each $i$, with equality holding only if e has at most one copy in $\hat{\mathrm{T}}_{\geqslant \lambda(A)+1}$.

We may assume that $i=1$ by symmetry. Let $B$ be the set displayed by the component of $T-\{e\}$ not containing $t_{2}$. Then

$$
\mathcal{w}(e)=\operatorname{dim}(\langle\mathrm{B}\rangle \cap\langle\mathrm{D}-\mathrm{B}\rangle)=\lambda(\mathrm{B})
$$

and

$$
\widehat{w}\left(e^{1}\right)=\operatorname{dim}(\langle A \cap B\rangle \cap\langle D-(A \cap B)\rangle)=\lambda(A \cap B) .
$$

So by the submodularity of $\lambda(\cdot)$,

$$
\begin{aligned}
\hat{w}\left(e^{1}\right)+\lambda(A \cup B) & \leqslant w(e)+\lambda(A) \\
& =w(e)+\kappa\left(D_{1}, D_{2}\right) \\
& \leqslant w(e)+\lambda(A \cup B) \\
\hat{w}\left(e^{1}\right) & \leqslant w(e)
\end{aligned}
$$

with equality holding only if $\lambda(A \cup B)=\lambda(A)$. Now suppose that $\hat{w}\left(e^{1}\right)=w(e)$. Then $\lambda(A \cup B)=\lambda(A)$ and $\lambda(A \cap B)=\lambda(B)$.

We argue that $A$ does not split $B$. Suppose otherwise. Then the choice of $A$ ensures that $A \cup B$ splits at least as many sets displayed by edges of $T$ as $A$ does. As $A \cup B$ does not split $B$, there exists a set $C$ displayed by an edge of $T$ that is split by $A \cup B$ but not by $A$. It follows that $B$ 
splits $C$ and $A \cap C$ is empty. As $A$ splits $B$, clearly $B-C$ is nonempty. As $B$ and $C$ are both displayed by edges of $T$ and $B$ splits $C$, necessarily $B \cup C=D$. So as $A \cap C$ is non-empty, $A \subseteq B$. Thus $\mathrm{D}_{1} \subseteq \mathrm{B}$. Moreover, $\mathrm{B} \subseteq \mathrm{D}-\mathrm{D}_{2}$ as $i=1$. So as $e_{1}$ and $e_{2}$ are not D-linked,

$$
\begin{aligned}
\kappa\left(D_{1}, D_{2}\right) & <\lambda(B) & & \\
& =\lambda(A \cap B) & & \\
& =\lambda(A) & & \text { as } A \subseteq B \\
& =\kappa\left(D_{1}, D_{2}\right) & & \text { by the choice of } A
\end{aligned}
$$

- a contradiction. Hence A does not split B.

So at least one of $A \cap B$ and $B-A$ is empty. As $\lambda(\varnothing)=0$, either $\lambda(A \cap B) \leqslant \lambda(A)$ or $\lambda(B-A) \leqslant \lambda(A)$. Now, $\hat{w}\left(e^{1}\right)=\lambda(A \cap B)$, and if $e^{2}$ exists, then

$$
\begin{aligned}
\hat{w}\left(e^{2}\right) & = \begin{cases}\lambda(A \cup B) & \text { if } e \in E\left(t_{1} T t_{2}\right), \text { and } \\
\lambda(B-A) & \text { otherwise }\end{cases} \\
& = \begin{cases}\lambda(A) & \text { if } e \in E\left(t_{1} T t_{2}\right), \text { and } \\
\lambda(B-A) & \text { otherwise. }\end{cases}
\end{aligned}
$$

Consequently $e$ has at most one copy in $\hat{T}_{\geqslant \lambda(A)+1}$.

Let

$$
K=\min \left\{k \in \mathbb{Z}_{>\lambda(A)} \mid\left(\forall l \in \mathbb{Z}_{>k}\right) e\left(T_{\geqslant l}\right)=e\left(\hat{T}_{\geqslant l}\right)\right\} .
$$

It follows from (4) that for every $l \in \mathbb{Z}_{\geqslant k}$, each edge of $T_{\geqslant l}$ has at most one copy in $\hat{T}_{\geqslant l}$. As $\hat{w}\left(t_{2}^{1} t_{1}^{2}\right)=\lambda(A)$, clearly $t_{2}^{1} t_{1}^{2} \notin E\left(\hat{T}_{\geqslant K}\right)$, so $e\left(T_{\geqslant k}\right) \geqslant e\left(\hat{T}_{\geqslant k}\right)$. The choice of $K$ ensures that $e\left(T_{\geqslant l}\right)=e\left(\hat{T}_{\geqslant l}\right)$ for every $l \in \mathbb{Z}_{>K}$. Moreover, $c\left(T_{\geqslant l}\right) \leqslant c\left(\hat{T}_{\geqslant l}\right)$ whenever $l \in \mathbb{Z}_{\geqslant k}$ has $e\left(T_{\geqslant l}\right)=e\left(\hat{T}_{\geqslant l}\right)$.

As $(\hat{T}, \hat{w}) \nless_{E}(T, w)$, it follows that $e\left(T_{\geqslant l}\right)=e\left(\hat{T}_{\geqslant l}\right)$ and $c\left(T_{\geqslant l}\right)=$ $c\left(\hat{T}_{\geqslant l}\right)$ for every $l \in \mathbb{Z}_{\geqslant k}$. In particular, $e\left(T_{\geqslant k}\right)=e\left(\hat{T}_{\geqslant k}\right)$, so $K=\lambda(A)+1$ 
by definition.

As $c\left(T_{\geqslant \lambda(A)+1}\right)=c\left(\hat{T}_{\geqslant \lambda(A)+1}\right)$, each connected component of $T_{\geqslant \lambda(A)+1}$ is copied entirely and as one connected component in $\hat{T}_{\geqslant \lambda(A)+1}$. In particular, this is true of the connected component of $T_{\geqslant \lambda(A)+1}$ containing $t_{1} T t_{2}$. But this is impossible, as $e_{i}$ has a copy only in $T^{i}$ for each $i$, and $t_{2}^{1} t_{1}^{2} \notin E\left(T_{\geqslant \lambda(A)+1}\right)$. Hence $(T, \sigma)$ is D-linked.

Although this theorem would provide an adequate foundation upon which to well- (or even better-) quasi-order matroids representable over a fixed finite field, it seems unlikely that it is "best possible." As the property of being linked concerns edge bags, while width concerns vertex bags, it is possible that every finite configuration has an optimal linked tree-decomposition (Conjecture 8.1.1). In particular, a more direct proof than that given (perhaps similar to those of [Tho90, BD02]) should be obtainable.

\subsection{Roundness and Tree-Decompositions}

This section focuses on the interactions between round configurations and tree-decompositions. Its results represent the first steps towards the lifting of Theorem 6.2.5 to infinite configurations in Chapter 7. The structure of our argument coincides with that of the proof of Theorem 1.1.6 given in [KT91]. Indeed, the next result is an analogue of (1.4) in [KT91].

Proposition 6.3.1. Let $\mathrm{D}$ be a configuration, let $\mathrm{N}$ be a finite-dimensional round subconfiguration of $\mathrm{D}$ and let $(\mathrm{T}, \sigma)$ be a tree-decomposition of $\mathrm{D}$. Then $\langle\mathrm{N}\rangle \leqslant$ $\sigma(\mathrm{t})$ for some $\mathrm{t} \in \mathrm{V}(\mathrm{T})$.

Proof. Let $\left\{\mathrm{b}_{1}, \ldots, \mathrm{b}_{\mathrm{n}}\right\} \subseteq \mathrm{N}$ be a basis for $\langle\mathrm{N}\rangle$. Define $\sigma^{\prime}: \mathrm{V}(\mathrm{T}) \rightarrow \mathcal{S}(\langle\mathrm{N}\rangle)$ by $\sigma^{\prime}(t)=\sigma(t) \cap\langle N\rangle$ for every $t \in V(T)$. Then $\left(T, \sigma^{\prime}\right)$ is a tree-decomposition of $\mathrm{N}$ by Proposition 6.1.8. Let

$$
\mathrm{T}_{i}=\mathrm{T}\left[\left\{\mathrm{t} \in \mathrm{V}(\mathrm{T}) \mid \mathrm{b}_{1}, \ldots, \mathrm{b}_{i} \in \sigma^{\prime}(\mathrm{t})\right\}\right]
$$


for each $i$. Interpolation ensures that each $T_{i}$ is a (possibly empty) subtree of $\mathrm{T}$.

We argue inductively that $\left|T_{n}\right| \geqslant 1$. As $V\left(T_{1}\right)=\left\{t \in V(T) \mid b_{1} \in \sigma^{\prime}(t)\right\}$, (TD1) ensures that $\left|T_{1}\right| \geqslant 1$. Suppose that $\left|T_{n-1}\right| \geqslant 1$. Then if $\left|T_{n}\right|=0$, $\mathrm{V}\left(\mathrm{T}_{\mathrm{n}-1}\right) \cap \mathrm{V}\left(\mathrm{T}^{\prime}\right)$ is empty, where $\mathrm{T}^{\prime}=\mathrm{T}\left[\left\{\mathrm{t} \in \mathrm{V}(\mathrm{T}) \mid \mathrm{b}_{\mathrm{n}} \in \mathrm{S}^{\prime}(\mathrm{t})\right\}\right]$. Let $e$ be the edge incident on $V\left(T^{\prime}\right)$ in the (unique) shortest path in $T$ between $\mathrm{V}\left(\mathrm{T}_{\mathrm{n}-1}\right)$ and $\mathrm{V}\left(\mathrm{T}^{\prime}\right)$, as shown in Figure 6.7.

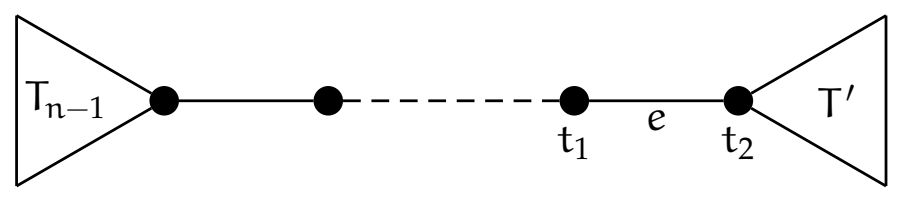

Figure 6.7: Proof of Proposition 6.3.1.

Let $T^{1} \geqslant T_{n-1}$ and $T^{2} \geqslant T^{\prime}$ be the connected components of $T-\{e\}$. Let $t_{j} \in e \cap V\left(T_{j}\right)$ and let $S_{j}=\sum_{t \in V\left(T^{j}\right)} \sigma^{\prime}(t)$ for each $j$.

Clearly $N \subseteq S_{1} \cup S_{2}$. If $b_{n} \in S_{1}$, then Interpolation would imply that $b_{n} \in \sigma^{\prime}\left(t_{1}\right)$, contradicting $t_{1} \notin V\left(T^{\prime}\right)$. Thus $b_{n} \notin S_{1}$, and so $S_{1} \lesseqgtr\langle N\rangle$. Similarly, if $b_{1}, \ldots, b_{n-1} \in S_{2}$, then Interpolation would imply that $b_{1}$, $\ldots, b_{n-1} \in \sigma^{\prime}\left(t_{2}\right)$, contradicting $t_{2} \notin V\left(T_{n-1}\right)$. Thus $b_{i} \notin S_{2}$ for some $i<n$, and so $S_{2} \lesseqgtr\langle N\rangle$, contradicting the roundness of $N$. It follows that $\left|T_{n}\right| \geqslant 1$, and so $\langle N\rangle \leqslant \sigma^{\prime}(t) \leqslant \sigma(t)$ for some $t \in V(T)$.

The next corollary is a special case of Proposition 6.3.1 that follows from Corollary 5.2.4.

Corollary 6.3.2. Let $\mathrm{D}$ be a configuration, let $\mathrm{U} \subseteq \mathrm{D}$ be a finite-dimensional vector space and let $(\mathrm{T}, \sigma)$ be a tree-decomposition of $\mathrm{D}$. Then $\mathrm{U} \leqslant \sigma(\mathrm{t})$ for some $\mathrm{t} \in \mathrm{V}(\mathrm{T})$.

The following example shows that Proposition 6.3.1 does not extend to infinite-dimensional round subconfigurations. 
Example 6.3.3. Let $V=\{\psi \mid(\exists n \in \omega) \psi: n \rightarrow \mathbb{R}\}$, let $B=\left\{b_{i} \mid i \in \omega\right\}$ be a basis for $V$ and let $D=B \sqcup X$, where $X=\left\{b_{i}+b_{j} \mid i, j \in \omega, i \neq j\right\}$. Then $\mathrm{D}$ is an infinite-dimensional Dowling clique, and thus round by Proposition 5.2.12.

Let $T$ be the ray $(\omega,\{\{i, i+1\} \mid i \in \omega\})$ and define $\sigma: \omega \rightarrow \mathcal{S}(V)$ by $\sigma(i)=\left\langle b_{0}, \ldots, b_{i}\right\rangle$ for every $i \in \omega$. Then $(T, \sigma)$ is clearly a tree-decomposition of $D$ having $D \nsubseteq \sigma(t)$ for every $t \in V(T)$.

Proposition 6.3.1 gives rise to following description of the tree-width of round configurations.

Proposition 6.3.4. Let $\mathrm{D}$ be a round configuration. Then $\operatorname{tw}(\mathrm{D})=\operatorname{dim}(\mathrm{D})$.

Proof. Corollary 6.1 .9 shows that $\operatorname{tw}(D) \leqslant \operatorname{dim}(D)$. Let $B \subseteq D$ be a basis for $\langle D\rangle$. It follows from Proposition 5.2.10 that $D /\left(B-B_{0}\right) \preccurlyeq D$ is round for every finite $\mathrm{B}_{0} \subseteq \mathrm{B}$, so

$$
\begin{aligned}
\operatorname{tw}(D) & \geqslant \operatorname{tw}\left(D /\left(B-B_{0}\right)\right) & & \text { by Coroll. 6.1.9 } \\
& \geqslant \operatorname{dim}\left(D /\left(B-B_{0}\right)\right) & & \text { by Prop. 6.3.1 } \\
& =\left|B_{0}\right| . & &
\end{aligned}
$$

Hence:

$$
\operatorname{tw}(D) \geqslant \sup \left\{\left|B_{0}\right|: B_{0} \subseteq B \text { is finite }\right\}=\#(B)=\operatorname{dim}(D) .
$$

Proposition 6.3.4 provides a natural lower bound on the tree-width of a configuration:

Proposition 6.3.5. Let $\mathrm{D}$ be a configuration. Then

$$
\operatorname{tw}(D) \geqslant \sup \{\operatorname{dim}(N) \mid N \preccurlyeq D \text { is round }\} .
$$

In particular, $\mathrm{tw}(\mathrm{D})=\infty$ if $\mathrm{D}$ has an infinite-dimensional round minor.

Proof. Combine Corollary 6.1.9 and Proposition 6.3.4. 
The following corollary is a special case of Proposition 6.3.5.

Corollary 6.3.6. Let D be a configuration. Then

$$
\operatorname{tw}(\mathrm{D}) \geqslant \sup \{\operatorname{dim}(\mathrm{U}) \mid \mathrm{U} \subseteq \mathrm{D} \text { is a subspace }\}
$$

In particular, $\mathrm{tw}(\mathrm{D})=\infty$ if $\mathrm{D}$ contains an infinite-dimensional vector space.

The next proposition is an analogue of (1.5) in [KT91] (see also Proposition 6.4.6).

Proposition 6.3.7. Let $(\mathrm{T}, \sigma)$ be a finite-width tree-decomposition of a configuration and let $\mathrm{D}=\bigcup_{\mathrm{t} \in \mathrm{V}(\mathrm{T})} \sigma(\mathrm{t})$. Let $\mathrm{U}, \mathrm{U}^{\prime} \subseteq \mathrm{D}$ be vector spaces and let $\mathrm{k} \in \mathrm{\omega}$. Then the following are equivalent:

(1) $\kappa_{\mathrm{D}}\left(\mathrm{U}, \mathrm{U}^{\prime}\right) \geqslant \mathrm{k}$.

(2) There exists a finite sequence $\mathrm{U}=\mathrm{U}_{1}, \ldots, \mathrm{U}_{\mathrm{n}}=\mathrm{U}^{\prime} \subseteq \mathrm{D}$ of finite-dimensional subspaces such that $\operatorname{dim}\left(\mathrm{U}_{i} \cap \mathrm{U}_{i+1}\right) \geqslant k$ for each $i=1, \ldots, n-1$.

Proof. Suppose that $(1)$ holds. As $(T, \sigma)$ has finite width, $U$ is finite-dimensional by Corollary 6.3.6. It follows from Corollary 6.3.2 that $U \leqslant \sigma(t)$ for some $t \in V(T)$. Similarly, $\mathrm{U}^{\prime} \leqslant \sigma\left(\mathrm{t}^{\prime}\right)$ for some $\mathrm{t}^{\prime} \in \mathrm{V}(\mathrm{T})$.

As $k\left(U, U^{\prime}\right) \geqslant k$,

$$
\min \left(\left\{\operatorname{dim}(U), \operatorname{dim}\left(U^{\prime}\right)\right\} \cup\left\{w(e) \mid e \in E\left(t T t^{\prime}\right)\right\}\right) \geqslant k
$$

Write $t T t^{\prime}=t, e_{1}, t_{1}, e_{2}, \ldots, e_{m}, t^{\prime}$. For each $i$, choose $S_{i} \leqslant \sigma\left(e_{i}\right)$ having dimension $k$. Applying Lemma 2.2.1 to each of the pairs $\left(U, S_{1}\right)$, $\left(S_{1}, S_{2}\right), \ldots,\left(S_{m}, U^{\prime}\right)$ yields $m+1$ finite sequences of subspaces, whose concatenation clearly satisfies (2).

Now suppose that (2) holds. Aiming for a contradiction, suppose that $\mathrm{K}\left(\mathrm{U}, \mathrm{U}^{\prime}\right)<\mathrm{k}$. Then there is a split $\left\{\mathrm{F}, \mathrm{F}^{\prime}\right\}$ of $\mathrm{D}$ having $\mathrm{U} \subseteq \mathrm{F}, \mathrm{U}^{\prime} \subseteq \mathrm{F}^{\prime}$ and $\operatorname{dim}\left(\langle F\rangle \cap\left\langle F^{\prime}\right\rangle\right)<k$. Consequently there is a least $i<n$ such that $\mathrm{U}_{i} \subseteq \mathrm{F}$ and $\mathrm{U}_{i+1} \subseteq \mathrm{F}^{\prime}$. But then $\mathrm{U}_{i} \cap \mathrm{U}_{i+1} \leqslant\langle\mathrm{~F}\rangle \cap\left\langle\mathrm{F}^{\prime}\right\rangle$, contradicting $\operatorname{dim}\left(U_{i} \cap U_{i+1}\right) \geqslant k$. Hence (1) holds. 


\subsection{Chordal Saturation and Tree-Decompositions}

This section investigates the relationships between chordally saturated configurations and tree-decompositions. In particular, it establishes a correspondence (Theorem 6.4.10) used in Chapter 7 to "locally encode" tree structure. This is a key ingredient in the proof of the main theorem.

Lemma 6.4.1. Let D be a finite-dimensional configuration that can be constructed recursively by pasting across subspaces, starting from subspaces. Then $\mathrm{D}$ has a finite tree-decomposition $(\mathrm{T}, \sigma)$ such that

(1) $\bigcup_{t \in V(T)} \sigma(t)=D$, and

(2) Width of $(\mathrm{T}, \sigma)=\operatorname{tw}(\mathrm{D})=\max \{\operatorname{dim}(\mathrm{U}) \mid \mathrm{U} \subseteq \mathrm{D}$ is a subspace $\}$.

Proof. It suffices to demonstrate that the following algorithm terminates and yields a finite tree-decomposition $(T, \sigma)$ of D satisfying (1) and (2).

Input: A finite-dimensional configuration D which can be constructed recursively by pasting across subspaces, starting from subspaces.

\section{Algorithm:}

$\langle 1\rangle$ Let $\mathrm{T}=\left(\left\{\mathrm{t}_{0}\right\}, \varnothing\right)$, let $\sigma: \mathrm{V}(\mathrm{T}) \rightarrow \mathcal{S}(\langle\mathrm{D}\rangle): \mathrm{t}_{0} \mapsto\langle\mathrm{D}\rangle$ and let $\psi: \mathrm{V}(\mathrm{T}) \rightarrow$ 2: $t_{0} \mapsto 0$.

$\langle 2\rangle$ If $\psi^{-1}\{0\}$ is non-empty, choose $t \in V(T)$ such that $\psi(t)=0$. Otherwise stop.

$\langle 3\rangle$ If $\sigma(t) \subseteq D$, redefine $\psi(t)=1$ and go to $\langle 2\rangle$. Otherwise, $D \cap \sigma(t)$ can be obtained by pasting some $\mathrm{D}_{1}$ and $\mathrm{D}_{2}$ together across the subspace $\mathrm{D}_{1} \cap \mathrm{D}_{2}$. In this case, modify $(T, \sigma, \psi)$ as follows.

(1) $\mathrm{V}(\mathrm{T}):=\mathrm{V}(\mathrm{T}) \sqcup\left\{\mathrm{t}_{1}, \mathrm{t}_{12}, \mathrm{t}_{2}\right\}$ 
(2) For every $t t^{\prime} \in E(T)$,

(2.1) if $\left\langle D_{1}\right\rangle \supseteq \sigma\left(t t^{\prime}\right) \nsubseteq\left\langle D_{2}\right\rangle$, redefine $E(T)=E(T) \sqcup\left\{t_{1} t^{\prime}\right\}$. Otherwise, if $\sigma\left(t t^{\prime}\right) \subseteq\left\langle D_{1}\right\rangle \cap\left\langle D_{2}\right\rangle$, redefine $E(T)=E(T) \sqcup\left\{t_{12} t^{\prime}\right\}$. Otherwise, redefine $E(T)=E(T) \sqcup\left\{t_{2} t^{\prime}\right\}$.

(2.2) $\mathrm{E}(\mathrm{T}):=\mathrm{E}(\mathrm{T})-\left\{\mathrm{tt}^{\prime}\right\}$

(3) $\mathrm{V}(\mathrm{T}):=\mathrm{V}(\mathrm{T})-\{\mathrm{t}\}$

(4) $E(T):=E(T) \sqcup\left\{t_{1} t_{12}, t_{12} t_{2}\right\}$

(5) $\sigma\left(t_{1}\right):=\left\langle D_{1}\right\rangle$ and $\sigma\left(t_{12}\right):=D_{1} \cap D_{2}$ and $\sigma\left(t_{2}\right):=\left\langle D_{2}\right\rangle$.

(6) $\psi\left(t_{1}\right):=0$ and $\psi\left(t_{12}\right):=1$ and $\psi\left(t_{2}\right):=0$.

Output: A finite tree-decomposition ( $T, \sigma)$ of D satisfying (1) and (2).

It is easy to see that if the algorithm terminates, then $T$ is a finite tree, $\sigma: \mathrm{V}(\mathrm{T}) \rightarrow \mathcal{S}(\langle\mathrm{D}\rangle)$ and (TD1) holds. Given that the algorithm terminates and that Interpolation holds, (1) and (2) clearly hold. So we demonstrate termination of the algorithm and Interpolation.

The algorithm begins with a single finite-dimensional subspace labelled by 0 . At each iteration, it either changes a 0 -label to 1 or replaces a 0-labelled subspace by two 0-labelled subspaces each having strictly smaller dimension. Consequently the algorithm terminates.

Clearly it suffices to demonstrate that Interpolation is preserved in $\langle 3\rangle$. So suppose that $\mathrm{T}$ is modified in $\langle 3\rangle$ as shown in Figure 6.8, the left-hand side of which represents a tree-decomposition $(T, \sigma)$ of $D$.

Then with the possible exceptions of $d_{1}$ and $d_{2}$, Interpolation holds for every edge of the new pair $(T, \sigma)$ represented by the right-hand side of Figure 6.8. It suffices by symmetry to demonstrate that Interpolation holds for $d_{1}$.

Let $A_{i}$ be the subspace associated with $T_{i}^{a}$ for each $i=1, \ldots, l$. Define 

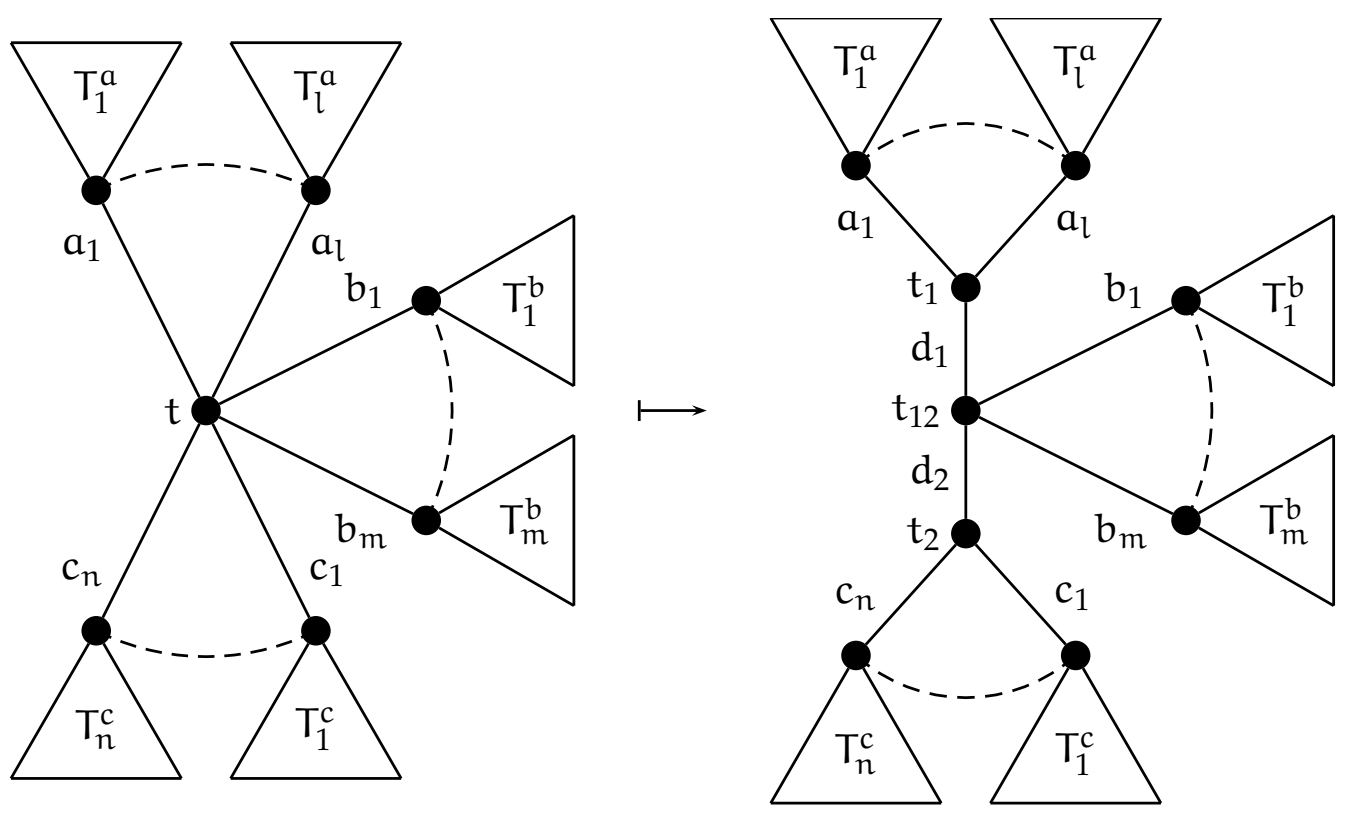

Figure 6.8: Surgery on the tree decomposition. The vertex $t$ is replaced by a 2-path, thus revealing a split of $D \cap \sigma(t)$.

$B_{1}, \ldots, B_{m}$ and $C_{1}, \ldots, C_{n}$ similarly, and let

$$
x \in\left\langle\bigcup_{i=1}^{l} A_{i} \cup D_{1}\right\rangle \cap\left\langle\bigcup_{j=1}^{m} B_{j} \cup \bigcup_{k=1}^{n} C_{k} \cup D_{2}\right\rangle .
$$

Then

$$
x=\sum_{i=1}^{l} x_{i}+w_{1}=\sum_{j=1}^{m} y_{j}+\sum_{k=1}^{n} z_{k}+w_{2}
$$

where $x_{i} \in A_{i}, y_{j} \in B_{j}, z_{k} \in C_{k}$ and $w_{h} \in\left\langle D_{h}\right\rangle$ for every $i, j, k$ and $h$. As Interpolation holds for each $b_{j}$, (6.4.1) shows that $y_{j} \in \sigma\left(b_{j}\right) \subseteq$ $\left\langle D_{1}\right\rangle \cap\left\langle D_{2}\right\rangle \subseteq\left\langle D_{2}\right\rangle$ for each $j$.

Moreover, as Interpolation holds for each $a_{i}$, (6.4.1) shows that $x_{i} \in$ 
$\sigma\left(a_{i}\right) \subseteq\left\langle D_{1}\right\rangle$ for each $i$. Symmetrically, $z_{k} \in\left\langle D_{2}\right\rangle$ for each $k$. Consequently $x \in\left\langle D_{1}\right\rangle \cap\left\langle D_{2}\right\rangle=D_{1} \cap D_{2} \subseteq \sigma\left(d_{1}\right)$, and so Interpolation holds for $d_{1}$.

Lemma 6.4.2. The configuration $\bigcup_{\mathrm{t} \in \mathrm{V}(\mathrm{T})} \sigma(\mathrm{t})$ is chordal for every tree-decomposition $(\mathrm{T}, \sigma)$.

Proof. Let $(T, \sigma)$ be a tree-decomposition, let $D=\bigcup_{t \in V(T)} \sigma(t)$ and let $\mathrm{C} \in \mathcal{C}(\mathrm{D})$ have size at least 4 . Let $\mathcal{T}$ be the collection of all finite subtrees of $T$ whose associated sets each contain $C$. Then whenever $f$ is a choice function for $\{\{t \in V(T) \mid c \in \sigma(t)\} \mid c \in C\}$,

$$
\bigcup_{\text {Distinct } c, c^{\prime} \in C} f(c) \operatorname{Tf}\left(c^{\prime}\right) \in \mathcal{T} \text {. }
$$

Consequently $\mathcal{T}$ is non-empty. Let $T^{\prime} \in \mathcal{T}$ be such that $\left|T^{\prime}\right|$ is minimal.

In order to establish the result, it suffices to show that $|C \cap \sigma(t)| \geqslant 2$ for some $t \in V\left(T^{\prime}\right)$. Clearly this is true if $T^{\prime}$ has only one vertex, so suppose otherwise. Then $L\left(T^{\prime}\right)$ is non-empty. Let $t \in L\left(T^{\prime}\right)$. If $|C \cap \sigma(t)| \leqslant 1$, then $\mathrm{T}^{\prime}-\{\mathrm{t}\} \in \mathcal{T}$ by Interpolation, contradicting the minimality of $\left|\mathrm{T}^{\prime}\right|$. Hence $|C \cap \sigma(t)| \geqslant 2$.

Proposition 6.4.3. Let $\mathrm{D}$ be a finite-dimensional configuration and let $w \in \omega$. Then the following are equivalent:

(1) $\mathrm{D}$ is chordally saturated and $w=\max \{\operatorname{dim}(\mathrm{U}) \mid \mathrm{U} \subseteq \mathrm{D}$ is a subspace $\}$.

(2) D can be constructed recursively by pasting along subspaces, starting from subspaces, and $w=\max \{\operatorname{dim}(\mathrm{U}) \mid \mathrm{U} \subseteq \mathrm{D}$ is a subspace $\}$.

(3) $\mathrm{D}$ has a finite tree-decomposition $(\mathrm{T}, \sigma)$ of width $w$ for which $\bigcup_{\mathrm{t} \in \mathrm{V}(\mathrm{T})} \sigma(\mathrm{t})=$ D.

Proof. Proposition 5.3.10 shows that (1) implies (2). Lemma 6.4.1 shows that (2) implies (3). Finally, Lemma 6.4.2 and Proposition 6.3.1 show that (3) implies (1). 
Lemma 6.4.4. Let $\mathrm{D}$ be a chordally saturated configuration, let $\pi$ determine a contraction of $\mathrm{D}$ by a finite linearly independent $\mathrm{I} \subseteq \mathrm{D}$ and let $\mathrm{U}, \mathrm{U}^{\prime} \subseteq \mathrm{D}$ be subspaces such that $\operatorname{dim}\left(\pi[\mathrm{U}] \cap \pi\left[\mathrm{U}^{\prime}\right]\right) \geqslant k \in \omega$. Then there exist subspaces $\mathrm{U}=\mathrm{U}_{1}, \ldots, \mathrm{U}_{\mathrm{n}}=\mathrm{U}^{\prime} \subseteq \mathrm{D}$ such that $\operatorname{dim}\left(\mathrm{U}_{\mathrm{i}} \cap \mathrm{U}_{\mathrm{i}+1}\right) \geqslant \mathrm{k}$ for each $\mathrm{i}=1$, $\ldots, n-1$.

Proof. Let $\mathrm{V}^{\prime} \leqslant \pi[\mathrm{U}] \cap \pi\left[\mathrm{U}^{\prime}\right]$ have dimension $\mathrm{k}$ and let $\mathrm{V}=\mathrm{V}^{\prime} \oplus\langle\mathrm{I}\rangle$. Then $\operatorname{dim}(\mathrm{V}) \leqslant \mathrm{k}+|\mathrm{I}| \in \omega$, so $\mathrm{D}^{\prime}:=\mathrm{D} \cap \mathrm{V}$ is a finite-dimensional chordally saturated configuration by Proposition 5.3.6.

Let $(T, \sigma)$ be a tree-decomposition of $\mathrm{D}^{\prime}$ as per Proposition 6.4.3.(3). Let $W_{1}=\mathrm{V} \cap \mathrm{U}$ and let $\mathrm{W}_{2}=\mathrm{V} \cap \mathrm{U}^{\prime}$. Then

$$
\begin{aligned}
\pi\left[\mathrm{W}_{1}\right] & =\operatorname{im}(\pi) \cap\left(\langle\mathrm{I}\rangle+\mathrm{W}_{1}\right) & & \text { by Prop. 2.2.5.(4) } \\
& =\operatorname{im}(\pi) \cap\left(\langle\mathrm{I}\rangle+\left(\left(\mathrm{V}^{\prime} \oplus\langle\mathrm{I}\rangle\right) \cap \mathrm{U}\right)\right) & & \\
& =\operatorname{im}(\pi) \cap\left(\left(\mathrm{V}^{\prime} \oplus\langle\mathrm{I}\rangle\right) \cap(\langle\mathrm{I}\rangle+\mathrm{U})\right) & & \text { by the Modular Law } \\
& =\pi\left[\mathrm{V}^{\prime}\right] \cap \pi[\mathrm{U}] & & \\
& =\mathrm{V}^{\prime} & & \text { as } \mathrm{V}^{\prime} \subseteq \pi[\mathrm{U}] .
\end{aligned}
$$

Similarly, $\pi\left[W_{2}\right]=V^{\prime}$.

As $W_{j} \subseteq D^{\prime}$ for each $j$, there exists $t_{j} \in V(T)$ such that $W_{j} \leqslant \sigma\left(t_{j}\right)$ by Corollary 6.3.2. Let $e \in E\left(t_{1} T t_{2}\right)$ and for each $j$ let $S_{j}$ be the subspace associated with the connected component of $T-\{e\}$ containing $t_{j}$. Then $W_{j} \leqslant S_{j}$, so $\pi\left[W_{j}\right] \leqslant \pi\left[S_{j}\right]$. Consequently $V^{\prime} \leqslant \pi\left[S_{1}\right] \cap \pi\left[S_{2}\right]$, and so $k \leqslant \operatorname{dim}\left(\pi\left[S_{1}\right] \cap \pi\left[S_{2}\right]\right) \leqslant w(e)$ by Proposition 6.1.8.

Thus every edge of $t_{1} T t_{2}$ has weight at least $k$. Write $t_{1} T t_{2}=t_{2}^{\prime}, t_{2}^{\prime} t_{3}^{\prime}$, $\ldots, t_{n-1}^{\prime}$. Let $U_{1}=U$, let $U_{i}=\sigma\left(t_{i}\right)$ for each $i=2, \ldots, n-1$ and let $U_{n}=$ $U^{\prime}$. Then $U_{1}, \ldots, U_{n} \subseteq D$ are subspaces such that $\operatorname{dim}\left(U_{i} \cap U_{i+1}\right) \geqslant k$ for each $i<n$.

Lemma 6.4.5. Let $\mathrm{D}$ be a chordally saturated configuration, let $\mathrm{k} \in \omega$ and let $\mathrm{U}_{1}, \ldots, \mathrm{U}_{\mathrm{n}} \subseteq \mathrm{D}$ be subspaces such that $\operatorname{dim}\left(\mathrm{U}_{\mathrm{i}} \cap \mathrm{U}_{\mathrm{i}+1}\right) \geqslant \mathrm{k}$ for each $\mathrm{i}=1$, 
$\ldots, \mathrm{n}-1$. Then there exists $\pi$ determining a contraction of $\mathrm{D}$ by a finite linearly independent $\mathrm{I} \subseteq \mathrm{D}$ such that $\operatorname{dim}\left(\pi\left[\mathrm{U}_{1}\right] \cap \pi\left[\mathrm{U}_{n}\right]\right) \geqslant \mathrm{k}$.

Proof. Let $\mathrm{u}_{i}^{\prime} \leqslant \mathrm{U}_{\mathrm{i}} \cap \mathrm{U}_{\mathrm{i}+1}$ have dimension $\mathrm{k}$ for each $\mathrm{i}<\mathrm{n}$. Let $\mathrm{V}_{1}=\mathrm{u}_{1}^{\prime}$, let $V_{n}=u_{n-1}^{\prime}$ and let $V_{i}=u_{i-1}^{\prime}+u_{i}^{\prime}$ for each $i=2, \ldots, n-1$. Set $\mathrm{V}=\sum_{i=1}^{n} V_{i}$. Then $\operatorname{dim}(\mathrm{V}) \leqslant 2 k n \in \omega$, so $\mathrm{D}^{\prime}:=\mathrm{D} \cap \mathrm{V}$ is a finite-dimensional chordally saturated configuration by Proposition 5.3.6.

Let $(T, \sigma)$ be a tree-decomposition of $\mathrm{D}^{\prime}$ as per Proposition 6.4.3.(3). Then for each $i$, as $V_{i} \subseteq D^{\prime}$, there exists $t_{i} \in V(T)$ such that $V_{i} \leqslant \sigma\left(t_{i}\right)$ by Corollary 6.3.2. If $t_{1}=t_{n}$, then Lemma 2.2.7 shows that there exists a $\pi$ as described. So suppose that $t_{1} \neq t_{n}$.

Let $e \in E\left(t_{1} T t_{n}\right)$ and let $S_{1}$ and $S_{2}$ be the subspaces displayed by $e$. Clearly each $V_{i}$ is contained in at least one $S_{j}$. Suppose that $w(e)<k$. Then each $V_{i}$ is contained in exactly one $S_{j}$ by Interpolation. As $V_{1} \leqslant S_{1}$ and $V_{n} \leqslant S_{2}$, there exists a least $i$ for which $V_{i} \leqslant S_{1}$ and $V_{i+1} \leqslant S_{2}$. But then $\operatorname{dim}\left(V_{i} \cap V_{i+1}\right) \leqslant \operatorname{dim}\left(S_{1} \cap S_{2}\right)=w(e)<k$, contradicting $\operatorname{dim}\left(V_{i} \cap\right.$ $\left.V_{i+1}\right) \geqslant k$. Consequently every edge of $t_{1} T t_{n}$ has weight at least $k$.

We argue inductively that there exists a finite sequence of projections whose application reduces this case to that of $t_{1}=t_{n}$. Let $e=t_{1} t_{1}^{\prime} \in$ $E\left(t_{1} T t_{n}\right)$. If $V_{1} \leqslant \sigma(e)$, simply replace $t_{1}$ by $t_{1}^{\prime}$ and let $\pi=i d_{\langle D\rangle}$ determine a contraction of $\mathrm{D}$ by $\varnothing$.

Otherwise, let $T_{1}$ be the component of $T-\{e\}$ containing $t_{1}$ and let $T_{2}$ be the other component of $T-\{e\}$. Let $S_{j}=\sum_{t \in V\left(T_{j}\right)} \sigma(t)$ for each $j$ and let $V_{1}^{\prime} \leqslant \sigma(e)$ contain $V_{1} \cap \sigma(e)$ and have dimension $k$. Then $\left(S_{1}, S_{2}, V_{1}, V_{1}^{\prime}\right)$ satisfies the hypotheses of Lemma 2.2.7, so there exist finite $X \subseteq D \cap \sigma\left(t_{1}\right)$ and a complement $\mathrm{U} \geqslant \mathrm{S}_{2}$ of $\langle\mathrm{X}\rangle$ in $\langle\mathrm{D}\rangle$ such that $\pi\left[\mathrm{V}_{1}\right] \leqslant \sigma(e)$ and $\operatorname{dim}\left(\pi\left[V_{1}\right]\right)=k$, where $\pi$ is projection of $\langle D\rangle$ onto $U$ along $\langle X\rangle$. Consequently we may replace $t_{1}$ by $t_{1}^{\prime}$.

It follows that there exists $\pi$ determining a contraction of $\mathrm{D}$ by some finite $X \subseteq D$ such that $\pi\left[V_{1}\right]=\pi\left[V_{n}\right] \leqslant \sigma\left(t_{n}\right) \subseteq \pi[D]$ and $\operatorname{dim}\left(\pi\left[V_{1}\right]\right)=k$. Finally, we note that contracting a finite set $X$ is the same as contracting a maximal independent subset of $X$, and that as $V_{1} \leqslant U_{1}$ and $V_{n} \leqslant U_{n}$, 
necessarily $\operatorname{dim}\left(\pi\left[\mathrm{U}_{1}\right] \cap \pi\left[\mathrm{u}_{n}\right]\right) \geqslant k$.

Combining Lemmas 6.4.4 and 6.4.5 yields the next proposition, an analogue of (1.5) in [KT91] (see also Proposition 6.3.7).

Proposition 6.4.6. Let $\mathrm{D}$ be a chordally saturated configuration, let $\mathrm{k} \in \mathrm{\omega}$ and let $\mathrm{U}, \mathrm{U}^{\prime} \subseteq \mathrm{D}$ be subspaces. Then the following are equivalent:

(1) There exist subspaces $\mathrm{U}=\mathrm{U}_{1}, \ldots, \mathrm{U}_{\mathrm{n}}=\mathrm{U}^{\prime} \subseteq \mathrm{D}$ such that $\operatorname{dim}\left(\mathrm{U}_{i} \cap\right.$ $\left.\mathrm{u}_{i+1}\right) \geqslant \mathrm{k}$ for each $\mathrm{i}=1, \ldots, \mathrm{n}-1$.

(2) There exists $\pi$ determining a contraction of $\mathrm{D}$ by a finite independent set such that $\operatorname{dim}\left(\pi[\mathrm{U}] \cap \pi\left[\mathrm{U}^{\prime}\right]\right) \geqslant \mathrm{k}$.

We now require a weaker form of interpolation that behaves more like that of tree-decompositions of graphs. Consequently we introduce the following notion.

Definition 6.4.7. Let $\mathrm{T}$ be a tree, let $\mathrm{U}$ be a vector space and let $\sigma: \mathrm{V}(\mathrm{T}) \rightarrow$ $\mathcal{S}(\mathrm{U})$. Set:

(PI) (Path Interpolation) $\sigma\left(t_{1}\right) \cap \sigma\left(t_{2}\right) \subseteq \sigma(t)$ for every $t_{1}, t_{2} \in V(T)$ and each $t \in V\left(t_{1} T t_{2}\right)$.

Clearly Interpolation is (strictly) stronger than Path Interpolation. The next lemma is a direct analogue of (2.1) in [KT91].

Lemma 6.4.8. Let D be an infinite-dimensional chordally saturated configuration for which $\sup \{\operatorname{dim}(\mathrm{U}) \mid \mathrm{U} \subseteq \mathrm{D}$ is a subspace $\} \in \omega$. Then there exist a tree $\mathrm{T}$ and a bijection $\sigma: \mathrm{V}(\mathrm{T}) \rightarrow\{$ Maximal subspaces contained in $\mathrm{D}\}$ such that $(\mathrm{T}, \sigma)$ satisfies Path Interpolation.

Proof. Let $V=\left\{S_{i} \mid i \in I\right\}$ be the collection of maximal subspaces contained in $\mathrm{D}$ and let $\mathrm{K}$ be the complete graph having vertex set $\mathrm{V}$. Define the weight of $S_{i} S_{j} \in E(K)$ to be $w\left(S_{i} S_{j}\right)=\operatorname{dim}\left(S_{i} \cap S_{j}\right)$. We construct a maximum-weight spanning tree $\mathrm{T}$ of $\mathrm{K}$ using the Axiom of Choice.

Let $w=\sup \{\operatorname{dim}(\mathrm{U}) \mid \mathrm{U} \subseteq \mathrm{D}$ is a subspace $\}$ and let $\mathrm{E}_{w}=\varnothing$. Given $\mathrm{E}_{w} \subseteq \cdots \subseteq \mathrm{E}_{\mathrm{i}+1}$, choose $\mathrm{E}_{\mathrm{i}} \subseteq \mathrm{E}(\mathrm{K})$ such that 
(1) $E_{i}$ is obtained from $E_{i+1}$ by adding a (possibly empty) set of edges of weight $i$,

(2) $E_{i}$ contains no subset inducing a cycle, and

(3) $E_{i}$ is maximal with respect to (1) and (2).

As every edge of $K$ has weight at most $w-1$, it follows that $E_{0}$ is the edge set of a spanning tree $T$ of $K$.

Let $\sigma=\mathrm{id}_{V}$ and suppose that Path Interpolation fails for $(T, \sigma)$. Then it fails for a minimal path $P=t_{1}, t_{1} t_{2}, t_{2}, \ldots, t_{m}$. Let $x \in\left(\sigma\left(t_{1}\right) \cap \sigma\left(t_{m}\right)\right)-$ $\bigcup_{i=2}^{m-1} \sigma\left(t_{i}\right)$ and let $k=\min \{w(e) \mid e \in E(P)\}$.

Let $\pi$ be a projection of $\langle D\rangle$ along $\langle x\rangle$ onto some complement $U$ of $\langle x\rangle$ in $\langle D\rangle$. Then $\pi$ determines a contraction of $D$ by $x$. As $x \notin \bigcup_{i=2}^{m-1} \sigma\left(t_{i}\right)$,

$$
\min \left\{\operatorname{dim}\left(\pi\left[\sigma\left(t_{i}\right)\right] \cap \pi\left[\sigma\left(t_{i+1}\right)\right]\right) \mid i<m\right\}=k
$$

It follows from Proposition 6.4.6 that there exists finite linearly independent $\mathrm{I} \subseteq \pi[\mathrm{D}]$ such that if $\pi^{\prime}$ is a projection of $\langle\mathrm{D}\rangle$ along $\langle\mathrm{I}\rangle$ onto $\mathrm{U}^{\prime} \oplus\langle x\rangle$ for some complement $\mathrm{U}^{\prime}$ of $\langle\mathrm{I}\rangle$ in $\mathrm{U}$, then

$$
\begin{aligned}
k & \leqslant \operatorname{dim}\left(\left(\pi^{\prime} \circ \pi\right)\left[\sigma\left(t_{1}\right)\right] \cap\left(\pi^{\prime} \circ \pi\right)\left[\sigma\left(t_{m}\right)\right]\right) \\
& =\operatorname{dim}\left(\left(\pi \circ \pi^{\prime}\right)\left[\sigma\left(t_{1}\right)\right] \cap\left(\pi \circ \pi^{\prime}\right)\left[\sigma\left(t_{m}\right)\right]\right) \\
& =\operatorname{dim}\left(\pi\left[\pi^{\prime}\left[\sigma\left(t_{1}\right)\right] \cap \pi^{\prime}\left[\sigma\left(t_{m}\right)\right]\right]\right)
\end{aligned}
$$

where the last step follows from $x \in \pi^{\prime}\left[\sigma\left(t_{1}\right)\right] \cap \pi^{\prime}\left[\sigma\left(t_{m}\right)\right]$. Consequently

$$
\operatorname{dim}\left(\pi^{\prime}\left[\sigma\left(t_{1}\right)\right] \cap \pi^{\prime}\left[\sigma\left(t_{m}\right)\right]\right) \geqslant k+1
$$

Now, $\pi^{\prime}$ determines a contraction of $\mathrm{D}$ by a finite linearly independent subset of $\left.\pi\right|_{\mathrm{D}} ^{-1} \mathrm{I}$. So by Proposition 6.4.6, there exists a sequence of subspaces $\sigma\left(t_{1}\right)=U_{1}, \ldots, U_{n}=\sigma\left(t_{m}\right) \subseteq D$ such that $\operatorname{dim}\left(U_{i} \cap U_{i+1}\right) \geqslant k+1$ for each $i<n$, contradicting the choice of $T$. Hence Path Interpolation holds. 
The properties of chordal saturation can be harnessed to strengthen this result, as shown by the next proposition.

Proposition 6.4.9. Let $\mathrm{D}$ be an infinite-dimensional chordally saturated configuration for which $\sup \{\operatorname{dim}(\mathrm{U}) \mid \mathrm{U} \subseteq \mathrm{D}$ a subspace $\} \in \omega$. Then $\mathrm{D}$ has an irredundant tree-decomposition whose vertex bags are (precisely) the maximal subspaces contained in $\mathrm{D}$.

Proof. Let $(T, \sigma)$ be as per Lemma 6.4.8. Suppose that Interpolation fails for $(T, \sigma)$. Then as linear combinations are finite, it fails for $\left(T^{\prime},\left.\sigma\right|_{V\left(T^{\prime}\right)}\right)$ for some finite subtree $T^{\prime}$ of $T$. We may assume without loss of generality that $\left|T^{\prime}\right|$ is minimal.

Write $L\left(T^{\prime}\right)=\left\{t_{1}, \ldots, t_{n}\right\}$ and let $t_{1}^{\prime}$ be the neighbour of $t_{1}$ in $T^{\prime}$. As Path Interpolation holds, necessarily $n \geqslant 3$ (see Figure 6.9).

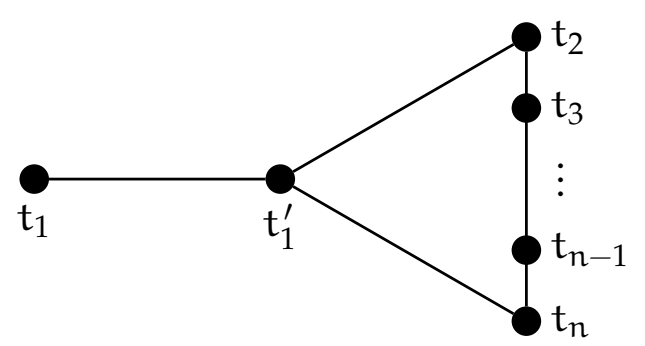

Figure 6.9: Proof of Proposition 6.4.9.

For each $i$, there exists $x_{i} \in \sigma\left(t_{i}\right)$ such that

$$
x_{1}=\sum_{i=2}^{n} \mu_{i} x_{i} \notin \sigma\left(t_{1}^{\prime}\right)
$$

for some non-zero scalars $\mu_{2}, \ldots, \mu_{n}$. Without loss of generality, $\mu_{2} x_{2}+$ $\mu_{3} x_{3} \in \mathrm{D}$ by Chordality. It follows by Saturation that $\mathrm{U}:=\left\langle x_{2}, x_{3}\right\rangle \subseteq \mathrm{D}$, so $\mathrm{U} \subseteq \sigma(\mathrm{t})$ for some $\mathrm{t} \in \mathrm{V}(\mathrm{T})$.

If $t \in V\left(T^{\prime}\right)$, then Path Interpolation in $T^{\prime}$ shows that Interpolation fails for $\left(T^{\prime \prime},\left.\sigma\right|_{V\left(T^{\prime \prime}\right)}\right)$ for some proper subtree $T^{\prime \prime}$ of $T^{\prime}$, contradicting the 
minimality of $\left|T^{\prime}\right|$. Consequently $t \notin V\left(T^{\prime}\right)$, so there exists a minimal path $P$ in $T$ linking $t$ to some $t^{\prime} \in V\left(T^{\prime}\right)$. But then Path Interpolation in $T^{\prime} \cup P$ shows that Interpolation fails for $\left(T^{\prime \prime},\left.\sigma\right|_{V\left(T^{\prime \prime}\right)}\right)$ for some proper subtree $\mathrm{T}^{\prime \prime}$ of $\mathrm{T}^{\prime}$, again contradicting the minimality of $\left|\mathrm{T}^{\prime}\right|$. Hence Interpolation holds for $(T, \sigma)$.

The following theorem establishs that chordally saturated configurations having finite tree-width have a "natural" tree structure. It is this ability to "locally encode" the global tree structure of a configuration that makes the argument of Theorem 7.1.5 possible. This role is fulfilled by (2.1) in [KT91].

Theorem 6.4.10. Let $\mathrm{D}$ be a configuration and let $w \in \omega$. Then the following are equivalent:

(1) D is chordally saturated and $w=\sup \{\operatorname{dim}(\mathrm{U}) \mid \mathrm{U} \subseteq \mathrm{D}$ is a subspace $\}$.

(2) $\mathrm{D}$ is chordally saturated and $w=\operatorname{tw}(\mathrm{D})$.

(3) D has a tree-decomposition $(\mathrm{T}, \sigma)$ of width $w$ for which $\bigcup_{\mathrm{t} \in \mathrm{V}(\mathrm{T})} \sigma(\mathrm{t})=\mathrm{D}$.

(4) D has an optimal irredundant tree-decomposition of width $w$ whose vertex bags are (precisely) the maximal subspaces contained in $\mathrm{D}$.

If $\mathrm{D}$ is finite-dimensional, these conditions are also equivalent to:

(5) D can be constructed recursively by pasting along subspaces, starting from subspaces, and $w=\sup \{\operatorname{dim}(\mathrm{U}) \mid \mathrm{U} \subseteq \mathrm{D}$ is a subspace $\}$.

(6) D can be constructed recursively by pasting along subspaces, starting from subspaces, and $w=\mathrm{tw}(\mathrm{D})$.

Proof. Let D be a configuration and let $w \in \omega$. We first show that $(1) \Rightarrow$ $(4) \Rightarrow(3) \Rightarrow(1)$. 
Suppose that (1) holds and that $\operatorname{dim}(D) \in \omega$. Then $D$ has a finite treedecomposition $(T, \sigma)$ of width $w$ for which $\bigcup_{t \in V(T)} \sigma(t)=D$ by Proposition 6.4.3. It follows from Proposition 6.1.11 that $\mathrm{D}$ has a finite irredundant tree-decomposition $\left(T^{\prime}, \sigma^{\prime}\right)$ of width $w$ for which $\bigcup_{t \in V\left(T^{\prime}\right)} \sigma^{\prime}(t)=D$. Clearly $\operatorname{tw}(\mathrm{D}) \leqslant w$, and $\operatorname{tw}(\mathrm{D}) \geqslant w$ by Corollary 6.3.6, so $\left(\mathrm{T}^{\prime}, \sigma^{\prime}\right)$ is optimal. As every vertex bag of $\left(T^{\prime}, \sigma^{\prime}\right)$ is a subspace contained in $D$, Corollary 6.3.2 shows that the vertex bags of $\left(\mathrm{T}^{\prime}, \sigma^{\prime}\right)$ are precisely the maximal subspaces contained in D.

Now suppose that $\operatorname{dim}(D)=\infty$. Then $D$ has an irredundant treedecomposition $(T, \sigma)$ whose vertex bags are (precisely) the maximal subspaces contained in D by Proposition 6.4.9. Necessarily $(T, \sigma)$ has width $w \geqslant \operatorname{tw}(D)$, and $\operatorname{tw}(D) \geqslant w$ by Corollary 6.3 .6$, so $\left(T^{\prime}, \sigma^{\prime}\right)$ is optimal. Thus (4) holds.

It is obvious that (4) $\Rightarrow(3)$, so suppose that (3) holds. Let $N \subseteq D$ be round. Then $\operatorname{dim}(N) \in \omega$ by Proposition 6.3.5, so $\langle N\rangle \subseteq \sigma(t) \subseteq D$ for some $t \in V(T)$ by Proposition 6.3.1. Consequently $D$ is saturated. Proposition 6.4.2 shows that $\mathrm{D}$ is chordal.

As every vertex bag of $(T, \sigma)$ is a subspace contained in D, necessarily $w \leqslant \sup \{\operatorname{dim}(\mathrm{U}) \mid \mathrm{U} \subseteq \mathrm{D}$ is a subspace $\}$. Corollary 6.3.6 shows that $\sup \{\operatorname{dim}(\mathrm{U}) \mid \mathrm{U} \subseteq \mathrm{D}$ is a subspace $\} \leqslant \operatorname{tw}(\mathrm{D})$. It follows that $w=$ $\sup \{\operatorname{dim}(\mathrm{U}) \mid \mathrm{U} \subseteq \mathrm{D}$ is a subspace $\}$, and so (1) holds.

We now demonstrate the equivalence of (1) and (2). First, suppose that (1) holds. Then D has an optimal tree-decomposition of width $w$ by the equivalence (1) $\Leftrightarrow(4)$, so $w=\operatorname{tw}(D)$. Thus (2) holds.

Now suppose that (2) holds. Then it follows from Corollary 6.3.6 that $\sup \{\operatorname{dim}(\mathrm{U}) \mid \mathrm{U} \subseteq \mathrm{D}$ is a subspace $\} \in \omega$. So D has an optimal tree-decomposition of width $\sup \{\operatorname{dim}(\mathrm{U}) \mid \mathrm{U} \subseteq \mathrm{D}$ is a subspace $\}$ by the equivalence $(1) \Leftrightarrow(4)$, and thus $w=\sup \{\operatorname{dim}(\mathrm{U}) \mid \mathrm{U} \subseteq \mathrm{D}$ is a subspace $\}$. Consequently (1) holds.

Now additionally suppose that $\mathrm{D}$ is finite-dimensional. Then the equivalence (1) $\Leftrightarrow$ (5) follows immediately from Proposition 6.4.3, and the 
equivalence (5) $\Leftrightarrow$ (6) follows from the equivalences $(2) \Leftrightarrow(1) \Leftrightarrow(5)$.

It is now easy to describe the tree-width of chordally saturated configurations.

Corollary 6.4.11. Let D be a chordally saturated configuration. Then

$$
\operatorname{tw}(\mathrm{D})=\sup \{\operatorname{dim}(\mathrm{U}) \mid \mathrm{U} \subseteq \mathrm{D} \text { is a subspace }\}
$$

Proof. If $\mathrm{tw}(\mathrm{D}) \in \omega$, this is immediate from Theorem 6.4.10. If $\mathrm{tw}(\mathrm{D})=$ $\infty$, then $\operatorname{dim}(\mathrm{D})=\infty$ by Corollary 6.1.9. It then follows from Proposition 6.4.9 that $\sup \{\operatorname{dim}(\mathrm{U}) \mid \mathrm{U} \subseteq \mathrm{D}$ is a subspace $\}=\infty$ also.

The following proposition extends Proposition 6.1.11 to tree-decompositions having finite width.

Proposition 6.4.12. Whenever a configuration has a tree-decomposition $(\mathrm{T}, \sigma)$ of width $w \in \omega$, it has an irredundant tree-decomposition $\left(\mathrm{T}^{\prime}, \sigma^{\prime}\right)$ of width $w$ for which $\operatorname{im}\left(\sigma^{\prime}\right) \subseteq \operatorname{im}(\sigma)$. Moreover, if $(T, \sigma)$ is finite, then so is $\left(T^{\prime}, \sigma^{\prime}\right)$.

Proof. If $(T, \sigma)$ is finite, this is precisely Proposition 6.1.11. So let $D$ be a configuration having an infinite tree-decomposition $(T, \sigma)$ of width $w \in$ $\omega$. Clearly $(T, \sigma)$ is also a tree-decomposition of $D^{\prime}:=\bigcup_{t \in V(T)} \sigma(t)$, so it follows from Theorem 6.4.10 that $\mathrm{D}^{\prime}$ has an irredundant tree-decomposition $\left(T^{\prime}, \sigma^{\prime}\right)$ of width $w$ whose bags are (precisely) the maximal subspaces contained in $\mathrm{D}^{\prime}$. Necessarily $\operatorname{im}\left(\sigma^{\prime}\right) \subseteq \operatorname{im}(\sigma)$, so the result follows from Proposition 6.1.3.

We close this section with an analogue of a compactness result for graphs discovered by Thomas, a proof of which is given by Thomassen on p. 410 of [Tho89b].

Theorem 6.4.13. Let $\mathrm{D}$ be a configuration and let $w \in \omega$. Then $\operatorname{tw}(\mathrm{D}) \leqslant w$ if and only if $\mathrm{tw}\left(\mathrm{D}_{0}\right) \leqslant w$ for every finite $\mathrm{D}_{0} \subseteq \mathrm{D}$. 
Proof. The forward implication follows immediately from Corollary 6.1.9, so we consider the reverse implication. Let $\mathrm{D}$ be a configuration for which every finite subconfiguration has tree-width at most $w \in \omega$ and let

$$
x=\left\{D^{\prime} \subseteq\langle D\rangle \mid D \subseteq D^{\prime} \text { and } \operatorname{tw}\left(D_{0}^{\prime}\right) \leqslant w \text { for every finite } D_{0}^{\prime} \subseteq D^{\prime}\right\} .
$$

Clearly $X$ is partially ordered by set inclusion. Let $y \subseteq X$ be totally ordered by set inclusion and let $\mathrm{D}^{\prime}=\bigcup y$. Suppose that $\mathrm{D}^{\prime} \notin X$. Then there exists finite $\mathrm{D}_{0}^{\prime} \subseteq \mathrm{D}^{\prime}$ such that $\operatorname{tw}\left(\mathrm{D}_{0}^{\prime}\right)>w$. As $\mathrm{D}_{0}^{\prime}$ is finite and $y$ is totally ordered, there exists $\mathrm{D}^{\prime \prime} \in \mathcal{y}$ containing $\mathrm{D}_{0}^{\prime}$, contradicting $\mathrm{D}^{\prime \prime} \in X$. Thus $\mathrm{D}^{\prime} \in X$, and so $y$ has an upper bound in $X$. It then follows from Zorn's Lemma that $X$ has a maximal element $\bar{D}$.

(1) $\overline{\mathrm{D}}$ is chordally saturated.

Let $\mathrm{N} \subseteq \overline{\mathrm{D}}$ be round and at most $w$-dimensional, let $x \in\langle\mathrm{N}\rangle$ and let $\mathrm{D}^{\prime}=\overline{\mathrm{D}} \cup\{x\}$. Clearly $\mathrm{D} \subseteq \mathrm{D}^{\prime} \subseteq\langle\mathrm{D}\rangle$. Let $\mathrm{D}_{0}^{\prime} \subseteq \mathrm{D}^{\prime}$ be finite. There exists finite round $\mathrm{N}_{0} \subseteq \mathrm{N}$ such that $\left\langle\mathrm{N}_{0}\right\rangle=\langle\mathrm{N}\rangle$ by Corollary 5.2.8. As $\mathrm{N}_{0} \cup\left(\mathrm{D}_{0}^{\prime}-\{x\}\right) \subseteq \overline{\mathrm{D}}$ is finite, clearly $\mathrm{tw}\left(\mathrm{N}_{0} \cup\left(\mathrm{D}_{0}^{\prime}-\{x\}\right)\right) \leqslant w$. It follows from Proposition 6.3.1 that $\operatorname{tw}\left(\mathrm{N}_{0} \cup \mathrm{D}_{0}^{\prime}\right) \leqslant w$ also, so tw $\left(\mathrm{D}_{0}^{\prime}\right) \leqslant$ $w$ by Corollary 6.1.9. Hence $\mathrm{D}^{\prime} \in \mathcal{X}$, so $\overline{\mathrm{D}}=\mathrm{D}^{\prime} \ni \mathrm{x}$ by the maximality of $\overline{\mathrm{D}}$. As the choice of $x$ was arbitrary, $\langle\mathrm{N}\rangle \subseteq \overline{\mathrm{D}}$.

Now suppose that $\mathrm{N} \subseteq \overline{\mathrm{D}}$ is round and at least $(w+1)$-dimensional. Let $\mathrm{B}_{1} \subseteq \mathrm{N}$ be linearly independent with $\left|\mathrm{B}_{1}\right|=w+1$. Extend $\mathrm{B}_{1}$ by $\mathrm{B}_{2} \subseteq \overline{\mathrm{D}}$ to a basis for $\langle\overline{\mathrm{D}}\rangle$ and let $\pi$ be the projection of $\langle\overline{\mathrm{D}}\rangle$ onto $\left\langle\mathrm{B}_{1}\right\rangle$ along $\left\langle\mathrm{B}_{2}\right\rangle$. Then $\pi$ determines a contraction of $\overline{\mathrm{D}}$ by $\mathrm{B}_{2}$.

$\pi[\mathrm{N}]$ is round by Corollary 5.2.10 and $(w+1)$-dimensional. It follows from Corollary 5.2.8 that there exists finite round $\mathrm{N}_{0} \subseteq \pi[\mathrm{N}]$ having dimension $w+1$. So by Proposition 4.4.7, $\mathrm{N}_{0} \subseteq \pi^{\prime}[\mathrm{N}]$ for some projection $\pi^{\prime}$ determining a contraction of $\overline{\mathrm{D}}$ by some finite $\mathrm{B}_{2}^{\prime} \subseteq \mathrm{B}_{2}$.

Let $X \subseteq N$ be finite and such that $\pi^{\prime}[X]=N_{0}$. Then $X \cup B_{2}^{\prime}$ is a finite subset of $\overline{\mathrm{D}}$. Corollary 6.1.9 and Proposition 6.3.4 imply that 
$\operatorname{tw}\left(X \cup B_{2}^{\prime}\right) \geqslant \operatorname{tw}\left(N_{0}\right)=\operatorname{dim}\left(N_{0}\right)=w+1$, contradicting $\bar{D} \in X$. Consequently $\overline{\mathrm{D}}$ contains no round subsets having dimension at least $w+1$, and so Saturation holds.

Suppose that $\bar{D}$ is not chordal. Then there exists $C=\left\{c_{1}, \ldots, c_{n}\right\} \in$ $\mathcal{C}(\bar{D})$ having size at least 4 such that $c_{i}+c_{j} \notin \bar{D}$ whenever $i \neq j$. It follows from the maximality of $\bar{D}$ that $\bar{D} \sqcup\left\{c_{i}+c_{j}\right\} \notin X$, so there exists finite $\mathrm{D}_{\mathfrak{i j}} \subseteq \overline{\mathrm{D}} \sqcup\left\{\mathrm{c}_{\mathfrak{i}}+\mathrm{c}_{\mathfrak{j}}\right\}$ such that $\operatorname{tw}\left(\mathrm{D}_{\mathfrak{i j}}\right)>\boldsymbol{w}$. Necessarily $c_{i}+c_{j} \in D_{i j}$. Consequently $D_{i j}^{\prime}:=D_{i j} \cup C \subseteq \bar{D} \sqcup\left\{c_{i}+c_{j}\right\}$ is finite and has tree-width at least $w+1$ by Corollary 6.1.9.

Let $D^{\prime}=\bigcup_{i \neq j}\left(D_{i j}^{\prime}-\left\{c_{i}+c_{j}\right\}\right)$. It follows from $D^{\prime}$ being a finite subset of $\overline{\mathrm{D}}$ that $\operatorname{tw}\left(\mathrm{D}^{\prime}\right) \leqslant w$. So there exists chordally saturated $\mathrm{D}^{\prime \prime} \supseteq \mathrm{D}^{\prime}$ such that $\operatorname{tw}\left(\mathrm{D}^{\prime \prime}\right) \leqslant w$ by Proposition 6.4.3. As $\mathrm{C} \subseteq \mathrm{D}_{i j}^{\prime}$ whenever $i \neq j, C \subseteq D^{\prime} \subseteq D^{\prime \prime}$, so $C \in \mathcal{C}\left(D^{\prime \prime}\right)$. It follows from the Chordality of $D^{\prime \prime}$ that $\mu c_{i}+\xi c_{j} \in D^{\prime \prime}$ for some $i \neq j$ and some non-zero scalars $\mu$ and $\xi$. So $c_{i}+c_{j} \in\left\langle c_{i}, c_{j}\right\rangle \subseteq D^{\prime \prime}$ by the Saturation of $D^{\prime \prime}$. Thus $\mathrm{D}_{i j}^{\prime} \subseteq \mathrm{D}^{\prime \prime}$, so $\operatorname{tw}\left(\mathrm{D}^{\prime \prime}\right) \geqslant \operatorname{tw}\left(\mathrm{D}_{\mathfrak{i j}}^{\prime}\right)>w$ by Corollary 6.1.9, contradicting $\operatorname{tw}\left(D^{\prime \prime}\right) \leqslant w$. Hence Chordality holds.

As $\bar{D}$ contains no round subsets having dimension at least $w+1$, it contains no subspaces having dimension at least $w+1$ by Corollary 5.2.4. Hence $\operatorname{tw}(\mathrm{D}) \leqslant \operatorname{tw}(\overline{\mathrm{D}}) \leqslant w$ by Corollaries 6.1.9 and 6.4.11.

Combining Corollary 6.1.9 with this result gives the following corollary.

Corollary 6.4.14. For every configuration D,

$$
\operatorname{tw}(\mathrm{D})=\sup \left\{\operatorname{tw}\left(\mathrm{D}_{0}\right) \mid \mathrm{D}_{0} \subseteq \mathrm{D} \text { is finite }\right\}
$$




\section{Chapter 7}

\section{Linked Decompositions: The Infinite Case}

In this chapter we finally establish the following theorem, which corresponds to (1.2) in [KT91].

Theorem 7.1.1. Every configuration having tree-width $w \in \omega$ has a linked tree-decomposition of width at most $2 w$.

We prove Theorem 7.1.1 by using Rado's Selection Lemma to "paste together" linked tree-decompositions of finite subconfigurations, yielding a linked tree-decomposition of the ambient configuration. As this approach cannot be applied directly to linked tree-decompositions, we begin by defining an equivalent object whose structure is "determined locally." This definition is an analogue of that given on p. 89 of [KT91].

Definition 7.1.2. Let $\mathcal{D}=(D, E, \ell)$ be a configuration having tree-width $w \in \omega$. Then an M-closure of $\mathcal{D}$ is a configuration $\widehat{\mathcal{D}}=(\widehat{\mathrm{D}}, \widehat{\mathrm{E}}, \widehat{\ell}) \geqslant \mathcal{D}$ satisfying:

(MC1) $\widehat{\mathcal{D}}$ is chordally saturated.

(MC2) $\widehat{D}$ contains no $(2 w+1)$-dimensional subspace. 
(MC3) Whenever $S_{1}$ and $S_{2}$ are distinct maximal subspaces contained in $\widehat{D}$ and $\Re_{i}$ is the union of those $\widehat{\ell}^{-1} S_{i}$-bridges of $\widehat{\mathcal{D}}$ disjoint from $\widehat{\ell}^{-1} S_{3-i}$ for each $i$,

$$
\kappa_{\mathcal{D}}\left(\ell^{-1}\left(S_{1} \sqcup \widehat{\ell}\left[\Re_{1}\right]\right), \ell^{-1}\left(S_{2} \sqcup \widehat{\ell}\left[\Re_{2}\right]\right)\right) \geqslant \kappa_{\widehat{\mathcal{D}}}\left(\widehat{\ell}^{-1} S_{1}, \widehat{\ell}^{-1} S_{2}\right) .
$$

In this definition, $(\mathrm{MC} 1)$ encodes tree structure, $(\mathrm{MC} 2)$ bounds width and (MC3) encodes the property of being linked. Note that (MC2) is chosen to correspond to the width bound in Theorem 6.2.5-were Conjecture 8.1.1 known to be true, " $w+1$ " would be used.

Unsurprisingly, it suffices in the context of M-closures to simply view configurations as subsets of their ambient spaces.

Proposition 7.1.3. Let $\mathcal{D}$ be a configuration having finite tree-width and point set $\mathrm{D}$. Then $\widehat{\mathcal{D}}$ having point set $\widehat{\mathrm{D}}$ is an M-closure of $\mathcal{D}$ if and only if $\widehat{\mathrm{D}}$ is an $\mathrm{M}$-closure of $\mathrm{D}$.

Proof. Combine Propositions 5.3.2, 5.1.11 and 4.5.2.

Of course, the last proposition does not encompass simplifications because (MC1) in principle forces the existence of subspaces having nontrivial parallel classes.

The next proposition establishes the equivalence of finite-width linked tree-decompositions and $M$-closures for configurations having finite treewidth. It corresponds to (2.2) in [KT91].

Proposition 7.1.4. Let D be a configuration having tree-width $w \in \omega$. Then $\mathrm{D}$ has a linked tree-decomposition of width at most $2 w$ if and only if it has an M-closure.

Proof. Let $(\mathrm{T}, \sigma)$ be a linked tree-decomposition of $\mathrm{D}$ having width at most $2 w$ and let $\widehat{D}=\bigcup_{t \in V(T)} \sigma(t) \geqslant D$. It follows from Theorem 6.4.10 that $\widehat{D}$ is chordally saturated and contains no $(2 w+1)$-dimensional subspace. Consequently statements (MC1) and (MC2) hold. 
Let $S_{1}$ and $S_{2}$ be distinct maximal subspaces contained in $\widehat{D}$. Then $S_{1}=\sigma\left(t_{1}\right)$ and $S_{2}=\sigma\left(t_{2}\right)$ for some distinct $t_{1}, t_{2} \in V(T)$ by Corollary 6.3.2. Let $P=t_{1} T t_{2}$. For each $i$, let $\Re_{i}$ be the union of the $S_{i}$-bridges of $\widehat{D}$ disjoint from $S_{3-i}$ and let $X_{i}$ be the set associated with the connected component of $T-E(P)$ containing $t_{i}$. Then it follows from Corollary 5.1.13 and Interpolation that $\mathfrak{R}_{i}=X_{i}-S_{i}$. Consequently

$$
\begin{aligned}
\mathrm{\kappa}_{\mathrm{D}}\left(\mathrm{D} \cap\left(\mathrm{S}_{1} \sqcup \Re_{1}\right), \mathrm{D} \cap\left(\mathrm{S}_{2} \sqcup \mathfrak{R}_{2}\right)\right) & =\mathrm{\kappa}_{\mathrm{D}}\left(\mathrm{D} \cap \mathrm{X}_{1}, \mathrm{D} \cap \mathrm{X}_{2}\right) \\
& =\min \{w(e) \mid e \in \mathrm{E}(\mathrm{P})\} \\
& \text { as }(\mathrm{T}, \sigma) \text { is D-linked } \\
& \geqslant \mathrm{\kappa}_{\widehat{\mathrm{D}}}\left(\mathrm{S}_{1}, \mathrm{~S}_{2}\right)
\end{aligned}
$$

where the last step follows from the fact that each $e \in E(P)$ displays subspaces that form a split of $S_{1}$ and $S_{2}$ in $\widehat{D}$. Hence (MC3) holds, and so $\widehat{\mathrm{D}}$ is an $M$-closure of $\mathrm{D}$.

Now let $\widehat{D}$ be an M-closure of $D$. Then $\widehat{D}$ has a tree-decomposition $(T, \sigma)$ of width at most $2 w$ for which $\bigcup_{t \in V(T)} \sigma(t)=\widehat{D}$ by Theorem 6.4.10. Moreover, $(T, \sigma)$ is a tree-decomposition of $D$ by Proposition 6.1.3. We verify that it is D-linked.

Let $t_{1}, t_{2} \in V(T)$ be distinct, let $P=t_{1} T t_{2}$ and let $X_{i}$ be the set associated with the component of $T-E(P)$ containing $t_{i}$ for each $i$. Write $P=t^{1}, t^{1} t^{2}, t^{2}, \ldots, t^{n}$ and let $k=\min \{w(e) \mid e \in E(P)\}$. Then $\sigma\left(t^{1}\right)$, $\ldots, \sigma\left(t^{n}\right)$ is a sequence of finite-dimensional subspaces contained in $\widehat{D}$ such that $\operatorname{dim}\left(\sigma\left(t^{j}\right) \cap \sigma\left(t^{j+1}\right)\right) \geqslant k$ for each $j<n$. It follows from Proposition 6.3 .7 that

$$
\kappa_{\widehat{D}}\left(\sigma\left(t_{1}\right), \sigma\left(t_{n}\right)\right) \geqslant k \text {. }
$$

So as $\widehat{D}$ is an M-closure of $D$,

$$
\mathrm{\kappa}_{\mathrm{D}}\left(\mathrm{D} \cap\left(\sigma\left(\mathrm{t}_{1}\right) \sqcup \Re_{1}\right), \mathrm{D} \cap\left(\sigma\left(\mathrm{t}_{2}\right) \sqcup \Re_{2}\right)\right) \geqslant \mathrm{k}
$$

where $\Re_{i}$ is the union of the $\sigma\left(t_{i}\right)$-bridges of $\widehat{D}$ disjoint from $\sigma\left(t_{3-i}\right)$ 
for each i. As $\bigcup_{t \in V(T)} \sigma(t)=\widehat{D}$, it follows from Corollary 5.1 .13 and Interpolation that $\sigma\left(t_{i}\right) \sqcup \Re_{i}=X_{i}$ for each $i$, so in fact

$$
\kappa_{D}\left(X_{1}, X_{2}\right) \geqslant k
$$

Hence $(T, \sigma)$ is D-linked.

The next theorem establishes the existence of $M$-closures for configurations having finite tree-width. It corresponds to (2.4) in [KT91].

Theorem 7.1.5. Every configuration having finite tree-width has an M-closure.

Proof. Let $\mathrm{D}$ be a configuration having tree-width $w \in \omega$. For every finite $X \subseteq\langle D\rangle$, let $\widehat{D}_{X}$ be an $M$-closure of $D_{X}:=D \cap X$. Define $f_{X}: X \rightarrow 2$ by

$$
f_{X}(x)= \begin{cases}1 & \text { if } x \in \widehat{D}_{X}, \text { and } \\ 0 & \text { otherwise }\end{cases}
$$

for every $x \in X$. Then by Rado's Selection Lemma, there exists $f:\langle D\rangle \rightarrow 2$ such that for every finite $X \subseteq\langle D\rangle$ there exists finite $Y \subseteq\langle D\rangle$ containing $X$ such that $\left.f\right|_{X}=\left.f_{Y}\right|_{X}$. Let $\widehat{D}=f^{-1}\{1\}$. Clearly $\widehat{D} \geqslant D$.

We first demonstrate Saturation. Let $N \subseteq \widehat{D}$ be round and at most $2 w$-dimensional. Then there exists finite round $N_{0} \subseteq N$ such that $\left\langle N_{0}\right\rangle=$ $\langle N\rangle$ by Corollary 5.2.8. Let $x \in\langle N\rangle$. Then $N_{0} \cup\{x\} \subseteq\langle D\rangle$ is finite, so there exists finite $Y \subseteq\langle D\rangle$ containing $N_{0} \cup\{x\}$ such that $\left.f\right|_{N_{0} \cup\{x\}}=\left.f_{Y}\right|_{N_{0} \cup\{x\}}$. Consequently $\mathrm{N}_{0} \subseteq \widehat{D}_{Y}$. It follows from the Saturation of $\widehat{D}_{Y}$ that $\left\langle\mathrm{N}_{0}\right\rangle \subseteq$ $\widehat{D}_{Y}$, so $x \in \widehat{D}_{Y}$. Thus $x \in \widehat{D}$. As the choice of $x$ was arbitrary, $\langle N\rangle \subseteq \widehat{D}$.

Now suppose that $\mathrm{N} \subseteq \widehat{\mathrm{D}}$ is round and at least $(2 w+1)$-dimensional. Let $\mathrm{B}_{1} \subseteq \mathrm{N}$ be linearly independent with $\left|\mathrm{B}_{1}\right|=2 w+1$. Extend $\mathrm{B}_{1}$ by $\mathrm{B}_{2} \subseteq \widehat{\mathrm{D}}$ to a basis for $\langle\widehat{\mathrm{D}}\rangle$ and let $\pi$ be the projection of $\langle\widehat{D}\rangle$ onto $\left\langle\mathrm{B}_{1}\right\rangle$ along $\left\langle B_{2}\right\rangle$. Then $\pi$ determines a contraction of $\widehat{D}$ by $B_{2}$.

$\pi[\mathrm{N}]$ is round by Corollary 5.2.10 and $(2 w+1)$-dimensional. It follows from Corollary 5.2.8 that there exists finite round $\mathrm{N}_{0} \subseteq \pi[\mathrm{N}]$ having di- 
mension $2 w+1$. So by Proposition 4.4.7, $\mathrm{N}_{0} \subseteq \pi^{\prime}[\mathrm{N}]$ for some projection $\pi^{\prime}$ determining a contraction of $\widehat{D}$ by some finite $\mathrm{B}_{2}^{\prime} \subseteq \mathrm{B}_{2}$.

Let $X \subseteq N$ be finite and such that $\pi^{\prime}[X]=N_{0}$. As $X \cup B_{2}^{\prime} \subseteq\langle D\rangle$ is finite, there exists finite $Y \subseteq\langle D\rangle$ containing $X \cup B_{2}^{\prime}$ such that $\left.f\right|_{X \cup B_{2}^{\prime}}=\left.f_{Y}\right|_{X \cup B_{2}^{\prime}}$. Consequently $X \cup B_{2}^{\prime} \subseteq \widehat{D}_{Y}$. Clearly $\pi^{\prime \prime}:=\left.\pi^{\prime}\right|_{\left\langle\widehat{D}_{Y}\right\rangle}$ determines a contraction of $\widehat{D}_{Y}$ by $B_{2}^{\prime}$, and $N_{0} \subseteq \pi^{\prime \prime}\left[\widehat{D}_{Y}\right]$. So there exists round $N^{\prime} \subseteq \widehat{D}_{Y}$ such that $\mathrm{N}_{0}=\pi^{\prime \prime}\left[\mathrm{N}^{\prime}\right]$ by Proposition 5.3.12.

Clearly $\operatorname{dim}\left(\mathrm{N}^{\prime}\right) \geqslant \operatorname{dim}\left(\mathrm{N}_{0}\right)=2 w+1$, so Saturation for $\widehat{D}_{Y}$ shows that $\widehat{D}_{Y}$ contains a $(2 w+1)$-dimensional subspace. Proposition 6.1 .3 and (MC2) then imply that $\operatorname{tw}(\mathrm{D}) \geqslant \operatorname{tw}\left(\mathrm{D}_{\mathrm{Y}}\right) \geqslant w+1$, contradicting $\mathrm{tw}(\mathrm{D})=$ $w$. Consequently $\widehat{D}$ contains no round subsets having dimension at least $2 w+1$, and so Saturation holds.

We now establish Chordality. Let $C \in \mathcal{C}(\widehat{D})$ have size at least 4 and let

$$
X=C \cup\left\{c+c^{\prime} \mid\left\{c, c^{\prime}\right\} \in[C]^{2}\right\}
$$

Clearly $X \subseteq\langle D\rangle$ is finite, so there exists finite $Y \subseteq\langle D\rangle$ containing $X$ such that $\left.f\right|_{X}=\left.f_{Y}\right|_{X}$. Consequently $C \in \mathcal{C}\left(\widehat{D}_{Y}\right)$. It follows from the Chordality of $\widehat{D}_{Y}$ that there exist distinct $c, c^{\prime} \in C$ and $d \in \widehat{D}_{Y}-C$ such that $\left\{c, c^{\prime}, d\right\}$, $\left(\mathrm{C}-\left\{\mathrm{c}, \mathrm{c}^{\prime}\right\}\right) \sqcup\{\mathrm{d}\} \in \mathcal{C}\left(\widehat{D}_{Y}\right)$. As $\left\{\mathrm{c}, \mathrm{c}^{\prime}, \mathrm{d}\right\}$ is round, $\left\langle\left\{c, c^{\prime}\right\}\right\rangle \subseteq \widehat{D}_{Y}$ by the Saturation of $\widehat{D}_{Y}$, so $c+c^{\prime} \in \widehat{D}_{Y}$. Thus $c+c^{\prime} \in \widehat{D}$. So as $\left\{c, c^{\prime}, c+c^{\prime}\right\}$ is round, $\left\langle\left\{c, c^{\prime}\right\}\right\rangle \subseteq \widehat{D}$ by the Saturation of $\widehat{D}$. Thus $d \in \widehat{D}$. Consequently $\left\{c, c^{\prime}, d\right\},\left(C-\left\{c, c^{\prime}\right\}\right) \sqcup\{d\} \in \mathcal{C}(\widehat{D})$, and so $\widehat{D}$ is Chordal. Thus (MC1) holds.

The statement (MC2) follows from the Saturation argument by the roundness of subspaces (see Corollary 5.2.4).

So we turn to (MC3). Let $S_{1}$ and $S_{2}$ be distinct maximal subspaces contained in $\widehat{D}$ and for each $i$ let $\Re_{i}$ be the union of those $S_{i}$-bridges of $\widehat{\mathrm{D}}$ disjoint from $\mathrm{S}_{3-i}$. As (MC1) and (MC2) hold, it follows from Theorem 6.4.10 that $\widehat{D}$ has a tree-decomposition $(T, \sigma)$ of finite width whose vertex bags are precisely the maximal subspaces contained in $\widehat{D}$. 
Thus $S_{1}=\sigma\left(t_{1}\right)$ and $S_{2}=\sigma\left(t_{2}\right)$ for some distinct $t_{1}, t_{2} \in V(T)$. Let $P=$ $t_{1} T t_{2}$. For each $t \in V(P)$, let $B_{t}$ be a basis for $\sigma(t)$ and let $N_{t}=B_{t} \sqcup\{b+$ $\left.b^{\prime} \mid\left\{b, b^{\prime}\right\} \in\left[B_{t}\right]^{2}\right\}$. Clearly $N_{t}$ is a finite Dowling clique spanning $\sigma(t)$. Let $X=\bigcup_{t \in V(P)} N_{t}$. As $X \subseteq\langle D\rangle$ is finite, there exists finite $Y \subseteq\langle D\rangle$ containing $X$ such that $\left.f\right|_{X}=\left.f_{Y}\right|_{X}$, so $X \subseteq \widehat{D}_{Y}$. It follows from Proposition 5.2.12 and the Saturation of $\widehat{D}_{Y}$ that $\bigcup_{t \in V(P)} \sigma(t) \subseteq \widehat{D}_{Y}$. Consequently

$$
\begin{aligned}
\kappa_{\widehat{D}}\left(S_{1}, S_{2}\right) & \leqslant \min \{w(e) \mid w \in E(P)\} & & \text { by Prop. 6.2.1 } \\
& \leqslant \kappa_{\widehat{D}_{Y}}\left(S_{1}, S_{2}\right) & & \text { by Prop. 6.3.7 } \\
& \leqslant \kappa_{D_{Y}}\left(D_{Y} \cap\left(S_{1} \sqcup \Re_{1}\right), D_{Y} \cap\left(S_{2} \sqcup \Re_{2}\right)\right) & & \text { by (MC3) } \\
& \leqslant \kappa_{D}\left(D_{Y} \cap\left(S_{1} \sqcup \Re_{1}\right), D_{Y} \cap\left(S_{2} \sqcup \Re_{2}\right)\right) & & \\
& \leqslant \kappa_{D}\left(D \cap\left(S_{1} \sqcup \Re_{1}\right), D \cap\left(S_{2} \sqcup \Re_{2}\right)\right) & & \text { by Prop. 4.5.3.(2). }
\end{aligned}
$$

Hence (MC3) holds for $\widehat{D}$.

Theorem 7.1.1 is now easily derived.

Proof of Theorem 7.1.1. Let $\mathrm{D}$ be a configuration having tree-width $w \in \omega$. Then D has an $M$-closure by Theorem 7.1.5 and thus has a linked treedecomposition of width at most $2 w$ by Proposition 7.1.4.

If Theorem 6.2.5 were strengthened to show that every finite configuration has an optimal linked tree-decomposition, this stronger result would easily lift to infinite configurations (see Conjecture 8.1.2). 


\section{Chapter 8}

\section{Discussion}

This thesis focused on linked tree-decompositions of configurations. We discuss some possible avenues of future research motivated by the results obtained.

Our main result (Theorem 7.1.1) is that every configuration having tree-width $w \in \omega$ has a linked tree-decomposition of width at most $2 w$. The proof given uses a finite version of this result (Theorem 6.2.5), new notions of bridges and chordality for configurations and a compactness argument. As the property of being linked concerns edge bags, while width concerns vertex bags, the factor of 2 in the width bound may be unnecessary.

Conjecture 8.1.1. Every finite configuration has an optimal linked tree-decomposition.

If Conjecture 8.1.1 were answered affirmatively, then the next conjecture would follow on replacing " $2 w$ " by " $w$ " in Definition 7.1.2 and the subsequent arguments.

Conjecture 8.1.2. Every configuration having finite tree-width has an optimal linked tree-decomposition.

It is natural to ask whether representability is necessary for the existence of linked tree-decompositions. As the proof of Theorem 6.2 .5 is 
essentially matroidal, it should be easy to show that every matroid having tree-width $w$ has a tree-decomposition of width at most $2 w$ (see p. 1120 of [HW06] for the definition of "tree-decomposition of a matroid"). However, answering the following conjecture would yield greater insight.

Conjecture 8.1.3. Every matroid has an optimal linked tree-decomposition.

As short proofs of Theorems 1.1.5 and 6.2.4 were given in [BD02] and [GGW02] respectively, there is probably a short proof answering Conjecture 8.1.3 affirmatively. Unfortunately, this does not appear to be a straightforward generalisation of the argument for finite graphs. A key difficulty is that the latter relies heavily on the use of paths, which have no matroidal analogue.

This may be an instance of a more general phenomenon: some matroidal results are first established for graphs via proofs relying on strictly graphic structure. Subsequent matroidal proofs can reveal enough structure to make further abstraction possible. For example, Theorem 6.2.4 is stated for connectivity functions. This suggests the following question.

\section{Question 8.1.4.}

(1) Are there notions of "connectivity function" and "tree-decomposition of a connectivity function" such that tree-decompositions of connectivity functions generalise both tree-decompositions of finite graphs and tree-decompositions of matroids?

(2) If so, is there a notion of "linked tree-decomposition of a connectivity function" for which the existence of linked tree-decompositions of connectivity functions can be established?

(3) If so, does this imply the existence of linked tree-decompositions of finite graphs and/or matroids?

Progress on this question might considerably further understanding of tree-decompositions. Certainly, unification of some of the various notions of "tree-decomposition" for different objects seems a worthwhile goal. 
Many questions about tree-decompositions remain open in the infinite case. In fact, no definition has yet been given for independence spaces.

Question 8.1.5. Is there a notion of "tree-decomposition of an independence space" such that tree-decompositions of independence spaces generalise tree-decompositions of matroids?

A suitable definition would have to avoid taking a difference of infinite ranks. It might be possible to achieve this by using some combination of bases, closure, restriction and contraction to reduce reliance on the rank function.

We demonstrated compactness of tree-width for configurations (Theorem 6.4.13). Given a positive answer to Question 8.1.5, it would be natural to ask whether this result extends to independence spaces in general.

Question 8.1.6. Is the tree-width of an independence space always the supremum of the tree-widths of its submatroids?

Answering Question 8.1.6 might require a notion of chordality for independence spaces (see Question 8.1.8).

Given positive answers to both Question 8.1.5 and Conjecture 8.1.3, it might be possible to answer the following question affirmatively using the proof strategy common to [KT91] and this thesis.

Question 8.1.7. Does every independence space having finite tree-width have an optimal linked tree-decomposition?

This would require purely matroidal formulations of the concepts developed in this thesis. While some of these (such as roundness) are already stated matroidally, others are not. In particular, a suitable substitute for chordal saturation would be required. This is an interesting question in its own right.

Question 8.1.8. Is there a notion of "chordal independence space" such that chordal matroids canonically correspond to tree-decompositions of matroids? 
That is, does Proposition 6.4.3 generalise to matroids? While a definition of chordality for independence spaces should clearly include the matroidal statement of (CS1), it is not immediately apparent what the second statement should be (or even whether one is necessary). If a density requirement is necessary, a suitable statement might involve modular pairs of flats. In this case, the ability to freely add points to a separation in a matroid until a modular pair is obtained might be useful for obtaining chordal closures.

It should be noted that a positive answer to Question 8.1.7 would be of little more use than Theorem 7.1.1 from the perspective of well-quasiordering. This is because finite certificates are necessary for a standard minimal bad sequence argument. In the case of independence spaces representable over a fixed finite field, these would arise from bounded tree-width forcing each vertex bag of a tree-decomposition to contain only finitely many points. Otherwise, there are no obvious alternative assumptions furnishing finite certificates. Note that finiteness of the field is necessary for well-quasi-ordering in the representable case (see p. 271 of [GGW02]).

However, Theorem 7.1.1 should make it possible to establish Conjecture 1.1.4. In fact, it is reasonable to expect that the following (stronger) conjecture could be established using Theorem 7.1.1 and an argument similar to that given in [Tho89a].

Conjecture 8.1.9. Every class of independence spaces representable over a fixed finite field and having bounded tree-width is well-behaved under the minor relation.

"Well-behaved" is a technical strengthening of better-quasi-ordered (and thus a strengthening of well-quasi-ordered) defined on p. 294 of [Tho89a]. 


\section{Bibliography}

[Ad106] Isolde Adler. Width Functions for Hypertree Decompositions. PhD thesis, University of Freiburg, Jan 2006. 100, 106

[BD02] Patrick Bellenbaum and Reinhard Diestel. Two short proofs concerning tree-decompositions. Combin. Probab. Comput., 11: 541-547, 2002. DOI: 10.1017/S0963548302005369. 121, 146

[BdM04] Joseph Bonin and Anna de Mier. T-uniqueness of some families of k-chordal matroids. Adv. in Appl. Math., 32:10-30, 2004. DOI: 10.1016/S0196-8858(03)00075-7. 89

[BF48] Garrett Birkhoff and Orrin Frink, Jr. Representations of lattices by sets. Trans. Amer. Math. Soc., 64:299-316, 1948. DOI: 10.2307/1990504. 18

[BG86] F. Barahona and M. Grötschel. On the cycle polytope of a binary matroid. J. Combin. Theory Ser. B, 40:40-62, 1986. DOI: 10.1016/0095-8956(86)90063-8. 89

[CFK04] Raul Cordovil, David Forge, and Sulamita Klein. How is a chordal graph like a supersolvable binary matroid? Discrete Math., 288:167-172, 2004. DOI: 10.1016/j.disc.2004.08.004. 89

[Die06] Reinhard Diestel. Graph Theory. Number 173 in Graduate Texts in Mathematics. Springer-Verlag, Berlin, third edition, 2006. ISBN: 3-540-26183-4. 1, 92, 104

[dMV03] Anna de Mier Vinué. Graphs and matroids determined by their Tutte polynomials. PhD thesis, Universitat Politécnica de Catalunya, 2003. 89 
[FF00] Claude-Alain Faure and Alfred Froölicher. Modern Projective Geometry. Number 521 in Mathematics and Its Applications. Kluwer, Dordrecht, The Netherlands, 2000. ISBN: 0-7923-65259. 33

[GGW02] James F. Geelen, A. M. H. Gerards, and Geoff Whittle. Branch-width and well-quasi-ordering in matroids and graphs. J. Combin. Theory Ser. B, 84:270-290, 2002. DOI: 10.1006/jctb.2001.2082. 3, 9, 60, 115, 117, 146, 148

[GGW03] James F. Geelen, A. M. H. Gerards, and Geoff Whittle. Disjoint cocircuits in matroids with large rank. J. Combin. Theory Ser. B, 87:270-279, 2003. DOI: 10.1016/S0095-8956(02)00010-2. 88

[GK06] Jim Geelen and Kasper Kabell. The Erdős-Pósa property for matroid circuits. Technical Report 1, Department of Mathematics, University of Aarhus, Jan 2006. ISSN: 1397-4076. 86

[Gła79] Kazimierz Głazek. Some old and new problems in the independence theory. Colloq. Math., 42:127Ü189, 1979. 18

[Gła03] Kazimierz Głazek. General notions of independence. In K. P. Shum, Z. X. Wan, and J. P. Zhang, editors, Advances in Algebra: Proceedings of the ICM Satellite Conference in Algebra and Related Topics, pages 112-128. World Scientific, Singapore, 2003. ISBN: 981-238-260-7. 18

[Grä79] G. Grätzer. Universal Algebra. Springer-Verlag, New York, second edition, 1979. ISBN: 0-387-90355-0. 18

[Gre75] Werner Greub. Linear Algebra. Number 23 in Graduate Texts in Mathematics. Springer-Verlag, New York, fourth edition, 1975. ISBN: 0-387-90110-8. 12

[GW03] James Geelen and Geoff Whittle. Cliques in dense GF(q)representable matroids. J. Combin. Theory Ser. B, 87:264-269, 2003. DOI: 10.1016/S0095-8956(02)00009-6. 80

[Hal76] Rudolf Halin. S-functions for graphs. J. Geometry, 8:171-186, 1976. DOI: 10.1007/BF01917434. 1 
[Hig69] D. A Higgs. Matroids and duality. Colloq. Math., 20:215-220, 1969. 20

[HK06] Marijn Heule and Oliver Kullmann. Decomposing clausesets: Integrating DLL algorithms, tree decompositions and hypergraph cuts for variable- and clause-based graph representations of CNF's. Report CSR 2-2006, University of Wales Swansea, 2006. 106

[Hli05] Petr Hliněný. Matroid tree-width and chordality. PDF Slides, Masaryk University in Brno, Faculty of Informatics, 2005. 89

[HOSG07] Petr Hliněný, Sang-il Oum, Detlef Seese, and Georg Gottlob. Width parameters beyond tree-width and their applicarions. Comput. J., 2007. DOI: 10.1093/comjnl/bxm052. 2

[HW06] Petr Hliněný and Geoff Whittle. Matroid tree-width. European J. Combin., 27:1117-1128, 2006. DOI: 10.1016/j.ejc.2006.06.005. $3,63,98,146$

[Kab06] Kasper Kabell. Extremal Matroid Theory and The Erdös-Pósa Theorem. PhD thesis, Department of Mathematical Sciences, University of Aarhus, Jan 2006. 81, 82, 85, 86

[KK82] J. Kahn and J. P. S. Kung. Varieties of combinatorial geometries. Trans. Amer. Math. Soc., 271:485-499, 1982. DOI: 10.2307/1998894. 80

[KT91] Igor Kř́ž and Robin Thomas. The Menger-like property of the tree-width of infinite graphs. J. Combin. Theory Ser. B, 52:86-91, 1991. DOI: 10.1016/0095-8956(91)90093-Y. 4, 88, 89, 114, 121, $124,131,134,139,140,142,147$

[Kun86] Joseph P. S. Kung. Growth rates and critical exponents of classes of binary combinatorial geometries. Trans. Amer. Math. Soc., 293:837-859, 1986. DOI: 10.2307/2000042. 88

[Lov05] László Lovász. Graph minor theory. Bull. Amer. Math. Soc. (N.S.), 43:75-86, 2005. DOI: 10.1090/S0273-0979-05-01088-8. 2

[Mar58] E. Marczewski. A general scheme of the notions of independence in mathematics. Bull. Acad. Polon. Sci. Ser. Sci. Math. Astr. Phys., 6:731-736, 1958. 18 
[NW68] C. St. J. A. Nash-Williams. On better-quasi-ordering transfinite sequences. Proc. Camb. Phil. Soc., 64:273-290, 1968. 2

[NW86] Giorgio Nicoletti and Neil White. Axiom systems. In Neil White, editor, Theory of Matroids, number 26 in Encyclopedia of Mathematics and its Applications, chapter 2, pages 29-44. Cambridge University Press, Cambridge, 1986. ISBN: 0-52130937-9. 24

[Ox178] James G. Oxley. Infinite matroids. Proc. London Math. Soc. (3), 37:259-272, 1978. 81

[Ox192] James Oxley. Infinite matroids. In Neil White, editor, Matroid Applications, number 40 in Encyclopedia of Mathematics and its Applications, chapter 3, pages 73-90. Cambridge University Press, Cambridge, 1992. ISBN: 0-521-38165-7. 19, 20, 25, $27,33,37$

[Ox104] James G. Oxley. Matroid Theory. Number 3 in Oxford Graduate Texts in Mathematics. Oxford University Press, New York, 2004. ISBN: 0-19-853563-5. 17, 20, 21, 26, 29, 39, 44, 50, 70, 81

[PP70] J. S. Pym and Hazel Perfect. Submodular functions and independence structures. J. Math. Anal. Appl., 30:1-31, 1970. 33

[Rad42] R. Rado. A theorem on independence relations. Q. J. Math., 13:83-89, 1942. DOI: 10.1093/qmath/os-13.1.83. 19

[Rad71] Richard Rado. A selection lemma. J. Combin. Theory, 10:176177, 1971. 15

[Rom05] Steven Roman. Advanced Linear Algebra. Number 135 in Graduate Texts in Mathematics. Springer, New York, second edition, 2005. ISBN: 0-387-24766-1. 13

[RS84] Neil Robertson and P. D. Seymour. Graph minors. III. Planar tree-width. J. Combin. Theory Ser. B, 36:49-64, 1984. 98

[RS90] Neil Robertson and P. D. Seynour. Graph minors. IV. TreeWidth and well-quasi-ordering. J. Combin. Theory Ser. B, 48: 227-254, 1990. DOI: 10.1016/0095-8956(90)90120-O. 1, 98, 115 
[RS95] Neil Robertson and P. D. Seymour. Graph minors. XIII. The disjoint paths problem. J. Combin. Theory Ser. B, 63:65-110, 1995. 2

[RS04] Neil Robertson and P. D. Seymour. Graph minors. XX. Wagner's conjecture. J. Combin. Theory Ser. B, 92:325-357, 2004. 1

[RST91] Neil Robertson, Paul Seymour, and Robin Thomas. Excluding infinite minors. Discrete Math., 95:303-319, 1991. DOI: 10.1016/0012-365X(91)90343-Z. 98

[Tho88] Robin Thomas. A counter-example to 'Wagner's conjecture' for infinite graphs. Math. Proc. Cambridge Philos. Soc., 103:5557, 1988. 2

[Tho89a] Robin Thomas. Well-quasi-ordering infinite graphs with forbidden finite planar minor. Trans. Amer. Math. Soc., 312, 1989. DOI: $10.2307 / 2001217.2,115,148$

[Tho89b] Carsten Thomassen. Configurations in graphs of large minimum degree, connectivity, or chromatic number. In Gary S. Bloom, Ronald L. Graham, and Joseph Malkevitch, editors, Combinatorial Mathematics Proceedings of the Third International Conference, number 555 in Ann. New York Acad. Sci., pages 402-412. New York Academy of Sciences, New York, 1989. 136

[Tho90] Robin Thomas. A Menger-like property of tree-width: The finite case. J. Combin. Theory Ser. B, 48:67-76, 1990. DOI: 10.1016/0095-8956(90)90130-R. 4, 115, 121

[Tut01] W. T. Tutte. Graph Theory. Number 21 in Encyclopedia of Mathematics and its Applications. Cambridge University Press, 2001. ISBN: 0-521-79489-7. 69

[Whi35] Hassler Whitney. On the abstract properties of linear dependence. Amer. J. Math., 57:509-533, 1935. 18 


\section{Notation}

\begin{tabular}{|c|c|}
\hline $\mathcal{D}^{\prime} \leqslant \mathcal{D}, 58$ & $\mathrm{G}\left[\mathrm{V}^{\prime}\right], 9$ \\
\hline $\mathrm{G}^{\prime} \leqslant \mathrm{G}, 8$ & $\gamma_{M}, 70$ \\
\hline $\begin{array}{l}\left(\mathrm{T}^{\prime}, w^{\prime}\right) \leqslant \mathrm{E}(\mathrm{T}, w), 9 \\
\mathrm{~N} \preccurlyeq M, 39\end{array}$ & $\mathcal{H}(M), 33$ \\
\hline$[X]^{2}, 86$ & $\mathcal{J}(\mathcal{D}), 51$ \\
\hline $\mathcal{B}(\mathcal{D}), 51$ & $\mathcal{J}(M), 18$ \\
\hline $\mathcal{B}(M), 18$ & $\mathrm{~K}_{\mathcal{D}}(\mathrm{X}, \mathrm{Y}), 65$ \\
\hline $\mathrm{C}(\mathcal{D}), 51$ & $\mathrm{~K}_{\mathrm{G}}(\mathrm{X}, \mathrm{Y}), 45$ \\
\hline$C(e, B), 22$ & $K_{M}(\lambda, 1), 4 J$ \\
\hline$c(G), 9$ & $\mathcal{L}(\mathcal{D}), 51$ \\
\hline $\mathcal{C}(M), 18$ & $\mathcal{L}(M), 18$ \\
\hline $\mathrm{cl}_{\mathrm{M}}, 33$ & $\mathrm{~L}(\mathrm{~T}), 8$ \\
\hline $\mathcal{D} / \times 60$ & $\lambda_{\mathcal{D}}(\mathrm{X}), 65$ \\
\hline $\mathcal{D} \backslash X, 57$ & $\lambda_{\mathcal{D}}(X, Y), 65$ \\
\hline$\Delta(\mathrm{G}), 8$ & $\lambda_{M}(X), 45$ \\
\hline$d_{c}(v), 8$ & $\lambda_{M}(X, Y), 45$ \\
\hline $\operatorname{dim}(\mathcal{D}), 50$ & $\mathcal{L} \mathcal{T} \mathcal{D}(\mathcal{D}), 114$ \\
\hline$\widetilde{\mathcal{D}}, 54$ & $M \cong M^{\prime}, 42$ \\
\hline & $M(G), 20$ \\
\hline $\mathrm{E}(\mathcal{D}), 50$ & $M / X, 36$ \\
\hline$E(G), 7$ & $M[\mathcal{D}], 51$ \\
\hline$e(G), 7$ & $M \backslash X, 23$ \\
\hline$E(M), 18$ & mod, 60 \\
\hline $\mathrm{E}_{\mathrm{G}}\left(\mathrm{V}^{\prime}\right), 8$ & $\widetilde{M}, 42$ \\
\hline $\mathcal{F}(\mathcal{D}), 51$ & $M \mid X, 23$ \\
\hline $\mathcal{F}(M), 33$ & $\mathrm{~N}_{\mathrm{G}}(\nu), 8$ \\
\hline $\mathrm{G}\left[\mathrm{E}^{\prime}\right], 9$ & $\omega(G), 89$ \\
\hline
\end{tabular}




$$
\begin{aligned}
& \mathcal{P}(E), 20 \\
& \mathcal{P}_{\text {fin }}(E), 29 \\
& r(M), 27 \\
& \rho_{M}, 41 \\
& r_{M}, 27 \\
& r_{M}(X), 27 \\
& \mathcal{S}(V), 11 \\
& \mathcal{T} \mathcal{D}(\mathcal{D}), 100 \\
& T_{\geqslant k}, 9 \\
& t T t^{\prime}, 8 \\
& \operatorname{tw}(\mathcal{D}), 99 \\
& U_{m, \beta}, 87 \\
& V(G), 7 \\
& V_{G}\left(E^{\prime}\right), 8 \\
& w(e), 99,109 \\
& w(t), 99
\end{aligned}
$$




\section{Index}

Axiom of choice, 15, 131

axioms

basis, 23, 24

chordal saturation, 90

circuit, 21

closure, 33

independent set, 17

M-closure, 139

rank, 27, 30

tree-decomposition, 99

bag (edge or vertex), 99

bases

of configurations, 51

of independence spaces, 18

basis exchange axiom, 24

branch-decomposition, 109

branch-width, 109

bridges, 71

characterisation of, 74, 76

choice function, 14

chord, 88

chordal, 88,90

chordality axiom, 90

chordally saturated, 90

circuits

of configurations, 51

of independence spaces, 18

clique, 87, 92

number, 89 closed set, 33

closure operator, 33

complement, 12

complete graph, 87, 92

configuration, 50

connected, 8

components

characterisation of, 74, 76

of configurations, 71

of graphs, 9

of independence spaces, 71

connectivity functions, 115

of configurations, 65

of independence spaces, 45

contractions

of configurations, 60

of independence spaces, 36

cubic tree, 8

cycle, 8

independence space, 20

degree/maximum degree, 8

deletions

of configurations, 57

of independence spaces, 23

dependent sets

of configurations, 51

of independence spaces, 18

dimension, 50

Dowling clique, 86, 91

joints of, 86 
edge set, 7

edge-weighted tree, 9

edge-weighting, 9

edges, 7

empty

graph, 7

separator, 71

endvertices, 8

equivalence

modulo a subspace, 60

of configurations, 52

finite

augmentation axiom, 17

character axiom, 18

configuration, 50

edge-weighted tree, 9

graph, 7

tree-decomposition, 100

flats

of configurations, 51

of independence spaces, 33

forest, 8

fundamental circuit, 22

geometric representation, 42

graph, 7

ground sets

of configurations, 50

of independence spaces, 18

hyperplanes

of independence spaces, 33

of vector spaces, 81, 95

J-subset, 25

incident, 7, 8

independence space, 18

graphic, 20

independent sets of configurations, 51

of independence spaces, 18

induced

configuration, 58

subgraph, 9

tree-decomposition, 109

interpolation axiom, 99

intersection of configurations, 57

irredundant tree-decomposition, 106

isomorphic

complements, 13

independence spaces, 42

leaf, 8

leaf-induced

tree-decomposition, 109

linked

D-, 114

tree-decomposition, 114

vertices, 8

loops

of configurations, 51

of graphs, 8

of independence spaces, 18

M-closure, 139

matroid, 18

meet properly, 102

middle basis axiom, 23

minor, 39

neighbour, 8

non-splitting, 80

non-trivial path, 113

optimal tree-decomposition, 99

order

of a configuration, 50

of a graph, 7 
of a split, 80

parallel

class, 41

edges, 8

pairs

of configurations, 51

of independence spaces, 18

pasting, 93

path, 8

interpolation axiom, 131

point set, 50

power set, 20

pre-independence space, 17

projection, 12

proper

flat, 33

meeting axiom, 103

Rado's selection lemma, 15, 25, 27, 37, 88, 139, 142

rank/rank function, 27

represented independence

space, 51

restrictions

of configurations, 57

of independence spaces, 23

round, 80

saturated, 90

saturation axiom, 90

separator, 71

set

associated with, 99

displayed by, 99

simple

configuration, 55

pre-independence space, 18

simplifications

of configurations, 54 of independence spaces, 42

spanning/spans, 33

split/splits, 80

strong circuit elimination axiom, 22

subconfiguration, 58

subgraph, 8

submodular function, 27

subspace

associated with, 99

displayed by, 98

transversal, 41

tree, 8

tree-decomposition, 99

tree-width, 99

triangles

of configurations, 51

of independence spaces, 18 , 87

triangulated, 88

uniform independence space, 87

union

of configurations, 57

of graphs, 8

vector independence space, 51

vertex

connectivity, 45

set, 7

vertices, 7

weak circuit elimination axiom, 21

width, 99, 109

Zorn's lemma, 15, 16, 23, 137 